Recht der Informationsgesellschaft

Roman Konertz | Raoul Schönhof

Das technische Phänomen

„Künstliche Intelligenz"

im allgemeinen Zivilrecht

Eine kritische Betrachtung im Lichte von Autonomie, Determinismus und Vorhersehbarkeit 
Recht der Informationsgesellschaft

herausgegeben von

Prof. Dr. Jörg Fritzsche, Universität Regensburg, Lehrstuhl für Bürgerliches Recht, Handels- und Wirtschaftsrecht Prof. Dr. Jürgen Kühling, LL.M., Universität Regensburg, Lehrstuhl für Öffentliches Recht, Immobilienrecht, Infrastrukturrecht und Informationsrecht

Prof. Dr. Gerrit Manssen, Universität Regensburg, Lehrstuhl für Öffentliches Recht, insbesondere deutsches und europäisches Verwaltungsrecht

Prof. Dr. Robert Uerpmann-Wittzack, Maître en droit, Universität Regensburg, Lehrstuhl für Öffentliches Recht und Völkerrecht

Band 47 
Roman Konertz | Raoul Schönhof

Das technische Phänomen

„Künstliche Intelligenz“

im allgemeinen Zivilrecht

Eine kritische Betrachtung im Lichte von

Autonomie, Determinismus und Vorhersehbarkeit 
BW-Förderkennzeichen: 036-170017.

Die Deutsche Nationalbibliothek verzeichnet diese Publikation in der Deutschen Nationalbibliografie; detaillierte bibliografische Daten sind im Internet über http://dnb.d-nb.de abrufbar.

\section{Auflage 2020}

๑ Roman Konertz | Raoul Schönhof

\section{Publiziert von}

Nomos Verlagsgesellschaft mbH \& Co. KG Waldseestraße 3-5 | 76530 Baden-Baden www.nomos.de

Gesamtherstellung: Nomos Verlagsgesellschaft mbH \& Co. KG Waldseestraße 3-5 | 76530 Baden-Baden

ISBN (Print): 978-3-8487-7675-7

ISBN (ePDF): 978-3-7489-1050-3

DOI: https://doi.org/10.5771/9783748910503

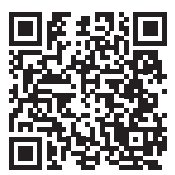

Onlineversion Nomos eLibrary

Die Bände 1 bis 33 sind im Lit-Verlag erschienen.

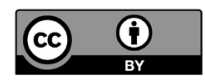

Dieses Werk ist lizensiert unter einer Creative Commons Namensnennung 4.0 International Lizenz. 


\section{Vorwort}

Das technische Phänomen der Künstlichen Intelligenz spielt auch im Recht eine immer größere Rolle. Nachdem wir uns intensiv mit den Auswirkungen dieses Phänomens auf das Patentrecht befasst haben, stellten wir im allgemeinen juristischen Schrifttum eine in Teilen bestehende Unschärfe oder Überschätzung des Phänomens fest. Daraus erwuchs das Ziel, Künstliche Intelligenz in einem für das Verständnis notwendigen Umfang für die Rechtswissenschaft darzulegen sowie darüber hinaus auf damit verbundene Probleme im allgemeinen Zivilrecht in Grundzügen einzugehen. Dieses Vorhaben überstieg allerdings schnell den Umfang eines üblichen Aufsatzes, sodass unsere Ergebnisse nun in der vorliegenden kleineren Schrift erscheinen. Der technische Teil kann dabei auch isoliert als allgemeine Beschreibung des Phänomens herangezogen werden, um mögliche Probleme auch in anderen Rechtsgebieten weiter zu untersuchen.

Dieses Werk erscheint mit freundlicher Unterstützung der FernUniversität in Hagen und des durch das Ministerium für Wirtschaft, Arbeit und Wohnungsbau Baden-Württemberg finanzierte KI-Fortschrittszentrum »Lernende Systeme« am Fraunhofer-Institut für Produktionstechnik und Automatisierung (IPA). Auch wäre diese Darstellung ohne die hervorragenden Forschungsmöglichkeiten an diesen Institutionen nicht möglich gewesen. Die Mitarbeit und der hiermit einhergehende Austausch an der Normungsroadmap »Künstliche Intelligenz« des Deutschen Instituts für Normung e.V. (DIN) hat uns zudem weiteren Einblick in das Phänomen gewährt.

Wir danken Herrn Professor Dr.-Ing. Marco Huber, Herrn Professor Dr. Sebastian Kubis und Herrn Professor Dr. Gerhard Wagner für ihre kritischen Kommentare und Anregungen in den verschiedenen Entwicklungsstadien. Zudem danken wir Frau Lisa Michalec, Frau Luzia Schuhmacher, Frau Nora Wahbé und Frau Uta Wichering, die uns bei der Erstellung des Manuskripts zur Seite standen. Ferner danken wir Herrn Professor Dr. Jörg Fritzsche, Herrn Professor Dr. Jürgen Kühling, Herrn Professor Dr. Gerrit Manssen und Herrn Professor Dr. Robert Uerpmann-Wittzack für die Aufnahme in diese Schriftenreihe. 
Inhaltsverzeichnis

$\begin{array}{ll}\text { Einleitung } & 15\end{array}$

A. Technische Phänomene in den Rechtswissenschaften 15

B. Stand der Forschung 17

C. Zweck und Gang der Darstellung 18

$\S 1$ Begriff und Übersetzungsproblem 21

$\S 2$ Wesen: Willen, Wissen und Verhalten 25

A. Problematik des Vergleichs zum Menschen 26

B. Definitionsversuche 30

C. Technologien der Künstlichen Intelligenz 32

I. Vom einfachen Schachcomputer zu AlphaZero 32

II. Methoden der Künstlichen Intelligenz im Überblick 34

1. Suchstrategien 34

2. Entscheidungsbäume 35

3. Clustering-Algorithmen 38

4. Fuzzylogiken 39

5. Semantic Web Systeme/Expertensysteme 40

6. Evolutionäre Algorithmen 42

7. Zwischenergebnis 44

III. Beispiel: (Künstliches) neuronales Netz 45

1. Modelldarstellung 45

2. Trainingsphase 47

3. Anwendungsphase 49

4. Sonderfall: Weiterlernende Systeme 49

5. Black-Box Konzept 51

IV. Multi-Agenten Systeme $\quad 52$

D. Vom Modell über den Algorithmus zur Computersoftware 52

I. Allgemeiner Algorithmus-Begriff 53

II. Randomisierte Algorithmen und Pseudozufall 54

III. Vom Algorithmus zur Computersoftware 55

IV. Folgen für das Wesen der Künstlichen Intelligenz 57 
E. Determinismus 58

I. Determinismus und Willensfreiheit in der Philosophie und im Recht

II. Determinismus, Determiniertheit und Terminierung in der Informatik

III. Konvergierender bzw. stochastischer Determinismus 60

F. Fehlerhaftigkeit, Erklärbarkeit und Verhaltensvorhersehbarkeit 62

I. Vom Sein und Sollen 64

II. Explainable AI Ansätze 65

G. Wissen und dessen Repräsentation 66

H. Zwischenergebnis 68

§ 3 Zurechenbarkeit und Folgen für das Vertrags- und Deliktsrecht $\quad 71$

A. Grundlagen der Zurechnungslehre 72

B. Von beteiligten Rechtssubjekten und -objekten 72

I. Künstliche Intelligenz und auf sie bezogene Entitäten als

Rechtsobjekt(e)

II. Beteiligte Rechtssubjekte 75

III. Rechtssubjektivierung von Künstlicher Intelligenz oder von auf sie bezogenen Entitäten $\quad 77$

IV. Rechtsobjekte als Zurechnungsausgangssubjekt $\quad 80$

C. Willenserklärungszurechnung 81

I. Dichotomie von Wille und Erklärung 83

II. Herkömmliche technische Einrichtungen 86

1. Verkörperung und Übertragung mithilfe von Computern 88

a) Vorliegen einer Willenserklärung $\quad 89$

b) Wirksamkeit 89

aa) Abgabe 90

bb) Zugang 93

2. Zuordnungsprobleme bei der Beteiligung von Dritten 94

3. Komplexe aus (vorprogrammierten) Willenserklärungen 96

a) Automatische Annahmen 97

b) Regelbasierte Willenserklärungen 99

III. Künstliche Intelligenz als Hilfsmittel 100

IV. Künstliche Intelligenz als autonomer »Erklärender« 104

1. Angebote durch autonome Systeme 105

2. Annahmen durch autonome Systeme 108

3. Vorliegen einer Blanketterklärung 111

4. Zwischenergebnis 111

$\begin{array}{ll}\text { V. Folgen } & 112\end{array}$ 
D. Haftungsrechtliche Verhaltens- und Handlungszurechnung 115

I. Haftung der Künstlichen Intelligenz für eigenes Verhalten 117

II. Haftung für eigenes Verhalten der Beteiligten 119

1. Verschuldensabhängige Haftung 119

a) Vorsatz 119

b) Fahrlässigkeit 120

2. Verschuldensunabhängige Haftung 125

III. Fremdzurechnung des Verhaltens der Künstlichen Intelligenz 126

1. Künstliche Intelligenz als Erfüllungsgehilfe 126

2. Künstliche Intelligenz als Verrichtungsgehilfe 127

IV. Kausalität 128

1. Äquivalenztheorie 129

2. Adäquanztheorie 130

3. Schutzzweck der Norm 131

V. Folgen 132

E. Wissenszurechnung 133

§ 4 Zusammenfassung und Thesen 135 


\section{Abkürzungsverzeichnis}

a.A.

Abs.

Alt.

ACM

$\mathrm{AcP}$

AI EDAM

ArchKrim

Art.

AtG

Aufl.

B.

BAG

Bd.

BeckRS

BGB

$\mathrm{BGH}$

BGHZ

BT-Drs.

BVerfG

BVerfGE

c.i.c.

CPS

CR

DIN

DNotZ

Einl.

et al.

evtl. andere Ansicht

Absatz

Alternative

Association for Computing Machinery

Archiv für die civilistische Praxis (Zeitschrift)

Artificial Intelligence for Engineering Design, Analysis and Manufacturing (Zeitschrift)

Archiv für Kriminologie (Zeitschrift)

Artikel

Atomgesetz

Auflage

Beschluss

Bundesarbeitsgericht

Band

Beck-Rechtsprechung

Bürgerliches Gesetzbuch

Bundesgerichtshof

Entscheidungen des Bundesgerichtshofs in Zivilsachen

Drucksache des Deutschen Bundestages

Bundesverfassungsgericht

Entscheidungen der amtlichen Sammlung des

Bundesverfassungsgerichts

culpa in contrahendo

Cyper-physisches System

Computer und Recht (Zeitschrift)

Deutsches Institut für Normung

Deutsche Notar-Zeitschrift

Einleitung

el alii/aliae

eventuell 

f./ff.
Fn.
Fordham L.Rev.
FS
gem.
ggf.
GPS
GRUR
folgender/folgende
Fußnote
Fordham Law Review
Festschrift
gemäß
gegebenenfalls
Global Positioning System
Gewerblicher Rechtsschutz und Urheberrecht (Zeitschrift)

GRUR-Prax

h.M.

Hs.

i.S.d.

i.V.m.

IEEE

InTeR

ITRB

J Med Syst

JA

Jura

JuS

JZ

K\&R

Kap.

KI

LG

LNCIS

Ls.

m.w.N

MedR
Gewerblicher Rechtsschutz und Urheberrecht, Praxis im Immaterialgüter- und Wettbewerbsrecht (Zeitschrift)

herrschende Meinung

Halbsatz

im Sinne des

in Verbindung mit

Institute of Electrical an Electronic Engineers

Zeitschrift zum Innovations- und Technikrecht

IT-Rechtsberater (Zeitschrift)

Journal of Medical Systems (Zeitschrift)

Juristische Arbeitsblätter (Zeitschrift)

Juristische Ausbildung (Zeitschrift)

Juristische Schulung (Zeitschrift)

JuristenZeitung (Zeitschrift)

Kommunikation und Recht (Zeitschrift)

Kapitel

Künstliche Intelligenz

Landgericht

Lecture Notes in Control and Information Sciences (Zeitschrift)

Leitsatz

mit weiteren Nachweisen

Medizinrecht (Zeitschrift) 
MMR

Mot.

NJW

NJW-RR

$\mathrm{Nr}$.

NZA-RR

NZG

OLG

PatG

PharmaR

Philosophical Magazine

ProdHaftG

Prot.

RG

$\mathrm{Rn}$.

RW

S.

s.

S. 0 .

s. u.

Ser.

sog.

St. Rsp.

StVG

U.

U.S.

UrhG

UWG
Zeitschrift für IT-Recht und Recht der Digitalisierung (vormals: Multimedia und Recht)

Motive

Neue Juristische Wochenschrift (Zeitschrift)

Neue Juristische Wochenschrift -

Rechtsprechungs-Report (Zeitschrift)

Nummer

Neue Zeitschrift für Arbeitsrecht -

Rechtsprechungs-Report

Neue Zeitschrift für Gesellschaftsrecht

Oberlandesgericht

Patentgesetz

Pharmarecht (Zeitschrift)

The London, Edinburgh, and Dublin Philosophical

Magazine and Journal of Science

Produkthaftungsgesetz

Protokolle

Reichsgericht

Randnummer

Zeitschrift für die rechtswissenschaftliche Forschung

Satz/Seite

siehe

siehe oben

siehe unten

Series

sogenannte/sogenannter/sogenanntes

Ständige Rechtsprechung

Straßenverkehrsgesetz

Urteil

United States

Urheberrechtsgesetz

Gesetz gegen den unlauteren Wettbewerb 
V.

VersR

vgl.

Vol.

WM

WRP

$\mathrm{xAI}$

z.B.

ZD

ZD-Aktuell

ZfPW

ZGE/IPJ

ZHR

ZRP

ZUM versus/vom/von

Versicherungsrecht (Zeitschrift)

vergleiche

Volume

Zeitschrift für Wirtschafts- und Bankrecht

Wettbewerb in Recht und Praxis (Zeitschrift)

Explainable Artificial Intelligence

zum Beispiel

Zeitschrift für Datenschutz

Newsdienst der Zeitschrift für Datenschutz

Zeitschrift für die gesamte Privatrechtswissenschaft

Zeitschrift für geistiges Eigentum

Zeitschrift für das gesamte Handels- und Wirtschaftsrecht

Zeitschrift für Rechtspolitik

Zeitschrift für Urheber- und Medienrecht 


\section{Einleitung}

\section{A. Technische Phänomene in den Rechtswissenschaften}

Technische Phänomene spielen für das Recht eine große Rolle: Einerseits bei der Frage, ob sie sich unter bestehende Normen subsumieren lassen, also ob und wie das bestehende Recht auf sie anwendbar ist; andererseits, ob sie durch rechtliche Vorschriften reguliert werden und diese somit selbst Einfluss auf den technischen Fortschritt nehmen. Daraus folgt, dass eine Wechselwirkung zwischen technischen Phänomenen und dem Recht besteht, sie sind somit Gegenstand der juristischen Betrachtung. ${ }^{1}$ Diese Wechselwirkung besteht nicht nur bei Rechtsgebieten, die schon durch ihre Natur eine gewisse Techniknähe aufweisen - wie beispielsweise die Immaterialgüterrechte ${ }^{2}$ - sondern auch im allgemeinen Zivilrecht, insbesondere bei der Rechtsgeschäftslehre und dem Haftungsrecht. In der Rechtsgeschäftslehre sind insbesondere die technischen Phänomene von Interesse, die neue Kommunikationsformen eröffnen, wie das Telefon, das Telefax, E-Mail oder Instantmessenger. Dennoch ist die Anwendung der seit dem Jahr 1900 kaum veränderten Regelungen der Rechtsgeschäftslehre - wenn auch mit dogmatischen Herausforderungen - für bisherige technische Phänomene bislang mit vertretbaren Ergebnissen möglich. ${ }^{3}$ Im Haftungsrecht dagegen sind die technischen Phänomene von Interesse, durch die sich das Risiko eines Schadens verwirklicht, beispielsweise Kraftfahrzeuge, Eisenbahnen, Flugzeuge oder Kernkraftwerke. Gerade das (außervertragliche) Haftungsrecht hat auf diese technischen Phänomene durch seine Weiterentwicklung auch auf gesetzlicher Ebene - reagiert. ${ }^{4}$

Bevor allerdings die gegenseitigen Einflüsse technischer Phänomene und des Rechts untersucht und diskutiert werden können und damit die technischen Phänomene für die juristische Betrachtung zugänglich sind, muss

1 Vgl. Roßnagel, MMR 2020, 222, 224 ff.; Specht, GRUR 2019, 253, 254 f.; Federrath, ZGE/IPJ 6 (2014), 271 ff.; Ehmann, AcP 188 (1988), 230, 259 ff.

2 Für technische Schutzrechte wie das Patentrecht gilt das unmittelbar, wie beispielsweise der Erfindungsbegriff des $\S 1$ Abs. 1 PatG zeigt: »Patente werden für Erfindungen auf allen Gebieten der Technik erteilt« oder für die Bestimmung des Stands der Technik i.S.d. § 4 PatG. Für das Urheberrecht vgl. Specht, GRUR 2019, 253, 255 ff.; Hofmann, ZGE/IPJ 8 (2016), 482 ff.

3 Vgl. Sutschet, NJW 2014, 1041; Wiebe, S. 1 ff.

4 Vgl. Wagner, in: Faust/Schäfer, S. 3 f. 


\section{Einleitung}

feststehen, welches technische Phänomen vorliegt und welche Eigenarten es hat. Nur so wird klar, was die Phänomene ausmacht und wie ihre Eigenschaften juristisch zu qualifizieren sind. Eine Herangehensweise besteht darin, das jeweilige Phänomen durch eine Definition abzugrenzen. Ungeklärt bleibt aber, ob überhaupt eine klare Definition möglich ist oder ob sich die Beschreibungen des Phänomens in unscharfen Umschreibungen erschöpfen. Ein bekanntes Beispiel ist der Versuch der Definition des technischen Phänomens der Eisenbahn durch das Reichsgericht im Jahre 1879, um sie für das Haftungsrecht zugänglich zu machen; es führte aus, dass:

»[e]ine Eisenbahn [...] ein Unternehmen [sei], gerichtet auf wiederholte Fortbewegung von Personen oder Sachen über nicht ganz unbedeutende Raumstrecken auf metallener Grundlage, welche durch ihre Konsistenz, Konstruktion und Glätte den Transport großer Gewichtmassen beziehungsweise die Erzielung einer verhältnismäßig bedeutenden Schnelligkeit der Transportbewegung zu ermöglichen bestimmt ist, und durch diese Eigenart in Verbindung mit den außerdem zur Erzeugung der Transportbewegung benutzten Naturkräften - Dampf, Elektrizität, tierischer oder menschlicher Muskeltätigkeit, bei geneigter Ebene der Bahn auch schon durch die eigene Schwere der Transportgefäße und deren Ladung usf. - bei dem Betriebe des Unternehmens auf derselben eine verhältnismäßig gewaltige, je nach den Umständen nur bezweckterweise nützliche oder auch Menschenleben vernichtende und menschliche Gesundheit verletzende Wirkung zu erzeugen fähig ist. « ${ }^{5}$

Dieser Definitionsversuch ist als »erschöpfende[n] und langstilige[n], ins Lächerliche abgleitende[n] Definition[en] «, ${ }^{6} \mathrm{zu}$ Recht in die Kritik geraten. Allerdings kann dieses Beispiel nicht darüber hinwegtäuschen, dass eine Eingrenzung von Phänomenen notwendig ist. Das beinhaltet auch eine Auseinandersetzung mit den technischen Eigenarten der Phänomene. Bezüglich der Eisenbahn kann man die rechtlichen Folgen des Phänomens beispielsweise nur dann thematisieren, wenn man in Grundzügen verstanden hat, was eine Eisenbahn überhaupt ist. In jüngster Vergangenheit ist gerade das - eigentlich jahrzehntealte - Phänomen der »Künstlichen Intelligenz« in den juristischen Fokus gerückt, wozu auch die sog. »autonomen Systeme« gezählt werden. ${ }^{7}$ Aus diesem technischen Phänomen bzw. dessen Eigenarten resultieren offenbar juristische Probleme, die einer näheren Betrachtung bedürfen. Auch für dieses Phänomen gilt, dass seine Eigenarten nur untersucht werden können, wenn zunächst feststeht, was das Phänomen

$5 \quad$ RG, U. v. 17.03.1879 - Rep. I. 23/80, RGZ 1, 247, 252.

6 Staudinger/Honsell, Einl. BGB, Rn. 141.

7 Vgl. Wahlster, Informatik-Spektrum 40 (2017), 409, 410. 
ausmacht. Daher müssen auch für die juristische Betrachtung des Phänomens »Künstliche Intelligenz« vorab die spezifischen Eigenarten der Technologie in einem notwendigen Maße dargelegt werden.

\section{B. Stand der Forschung}

Im juristischen Schrifttum besteht eine kaum noch zu überblickende Vielfalt an Literatur, die sich mit juristischen Fragen rund um die Künstliche Intelligenz befasst. Es werden neben spezieller Verwendung von Künstlicher Intelligenz als virtuellem Polizei-Avatar, ${ }^{8}$ zur Simulation des Körpers im Rahmen der Zulassung von Medikamenten oder zur Verbesserung von Medizinprodukten, ${ }^{9}$ zur Erleichterung von Entscheidungen in Unternehmen ${ }^{10}$ zur Suizidprävention im Strafvollzug ${ }^{11}$ oder im Bereich LegalTech $^{12}$ auch die Folgen der Handlungen von Künstlicher Intelligenz beleuchtet, beispielsweise hinsichtlich der schöpferischen Tätigkeit durch Künstliche Intelligenz im Urheber- und Patentrecht. ${ }^{13}$ Im allgemeinen Zivilrecht finden sich bereits - teilweise ausführliche - Untersuchungen zu den Auswirkungen auf die Rechtsgeschäftslehre und die Haftung ${ }^{14}$ oder zur Rechtsfähigkeitsproblematik. ${ }^{15}$ Auch bestehen genauere Untersuchungen zum Haftungsrecht, ${ }^{16}$ insbesondere zum Delikts- oder Produkthaftungsrecht. ${ }^{17}$ Monografisch hat sich insbesondere Grapentin mit den Fragen der Auswirkungen auf Vertragsschluss und vertragliche Haftung befasst. ${ }^{18}$

\footnotetext{
$8 \quad$ Vgl. Stoklas, ZD-Aktuell 2018, 06363.

9 Vgl. Dettling/Krüger, PharmaR 2018, 513, $515 \mathrm{ff}$.

10 Vgl. Weber/Kiefner/Jobst, NZG 2018, 1131 ff.; Söbbing, InTeR 2018, 64 ff.

11 Vgl. Esser/Reißmann, JZ 2019, 975 ff.

12 Vgl. Hoch, AcP 219 (2019), 646 ff.; v. Bünau, in: Breidenbach/Glatz, Rn. 25 ff.; v. Graevenitz, ZRP 2018, 238.

13 Vgl. Gomille, JZ 2019, 969 ff.; Hauck/Cevc, ZGE/IPJ 11 (2019), 135 ff.; Hetmank/ Lauber-Rönsberg, GRUR 2018, 574 ff.; Konertz/Schönhof, ZGE/IPJ 10 (2018), 379 ff.; Lauber-Rönsberg, GRUR 2019, 244 ff.; wissenschaftlich unsauber auch bei Nägerl/Neuburger/Steinbach, GRUR 2019, $336 \mathrm{ff.}$

14 Hacker, RW 2018, 234 ff.; Teubner, AcP 218 (2018), 155 ff.; Müller-Hengstenberg/Kirn, MMR 2014, 307.

15 Schirmer, JZ 2019, 711 ff.; Wagner, 88 Fordham L.Rev. 591, 595 ff. (2019); Schirmer, JZ 2016, 660 ff.

16 Klingbeil, JZ 2019, 718, 723; Zech, ZfPW 2019, 198; Denga, CR 2018, 69; Spindler, CR 2015, 766; Zech, in: Gless/Seelmann, S. 163 ff.

17 Wagner, AcP 217 (2017), 707 ff.; Wagner, in: Faust/Schäfer, S. 1 ff.

18 Grapentin, S. 1 ff.
} 
Söbbing ${ }^{19}$ hat allgemein die rechtlichen Fragen der Künstlichen Intelligenz untersucht, wobei auch diverse Nebengebiete angerissen werden. Auch Reusch und Weidner ${ }^{20}$ haben sich allgemein mit rechtlichen Fragen von möglichen, zukünftigen Technologien befasst, was eine allgemeine Untersuchung der Thematik und deren Folgen beinhaltet, wobei auch weitere Themengebiete erfasst werden, die mit dem technischen Phänomen der Künstlichen Intelligenz nicht im Zusammenhang stehen. Bei der Frage der Rechtsfähigkeit entsprechender KI-Systeme ist noch die Monografie von Mayinger ${ }^{21} \mathrm{zu}$ erwähnen, die grundlegend die e-Person erörtert.

Im Ganzen lässt sich allerdings feststellen, dass Teile der Literatur auf das technische Phänomen nicht weiter eingehen und die Probleme generisch mit unterstellten Annahmen untersuchen, die zwar durchaus zutreffend sein können, allerdings dann entsprechendes Vorwissen beim Leser verlangen. Der Teil der Literatur, der sich an den technischen Implikationen versucht, beinhaltet in der Darstellung des Öfteren Ungenauigkeiten oder die tatsächlichen Möglichkeiten einer Künstlichen Intelligenz werden teilweise überschätzt. ${ }^{22}$ Auch wird Künstliche Intelligenz mit in Teilen verwandten Konzepten wie »Deep-Learning « oder $»$ Big-Data $\ll^{23}$ verbunden, aber auch mit abstrakteren Phänomenen wie »künstlichem Bewusstsein «. ${ }^{24}$ Aus diesem Grunde besteht bereits ein Defizit bei der rechtstatsächlichen Betrachtung, sodass eine vertiefte, aber verständliche Darlegung des technischen Phänomens der Künstlichen Intelligenz angebracht ist und sich die Frage stellt, ob die bisherigen Ansätze den rechtstatsächlichen Gegebenheiten gerecht werden.

\section{Zweck und Gang der Darstellung}

Diese Untersuchung hat zwei grundsätzliche Ziele: Erstens soll das allgemeine, aber notwendige Verständnis über das technische Phänomen »Künstliche Intelligenz« in den Rechtswissenschaften gestärkt werden, sodass das Phänomen richtig verstanden wird und keine Fehlschlüsse oder Überinterpretationen stattfinden. Diese Darlegungen können auch isoliert

19 Söbbing/Söbbing, S. 1 ff.

20 Reusch/Weidner, Rn. 1 ff.

21 Mayinger, S. 1 ff.

22 Im Ergebnis ebenso v. Bünau, in: Breidenbach/Glatz, Rn. $1 \mathrm{ff}$.

23 Dettling/Krüger, PharmaR 2018, 513, 514; Söbbing/Spindler, S. V.

24 Dettling/Krüger, PharmaR 2018, 513, 514 mit Verweis auf Tegmark, S. 419 ff. 
als Grundlage für weitere Untersuchungen der rechtlichen Folgen in anderen Rechtsgebieten dienen. Zweitens sollen die juristischen Probleme, die im allgemeinen Zivilrecht durch das technische Phänomen möglicherweise entstehen, in ihren Grundzügen erörtert werden. Das soll vor allem anhand der Rechtsgeschäftslehre und dem Haftungsrecht geschehen, wobei bei der Untersuchung auf die Zuordnung von Willenserklärungen, haftungsrelevantem Verhalten und Wissen abgestellt wird.

$\mathrm{Zu}$ Beginn wird der Begriff »Künstliche Intelligenz« kurz aus sprachlicher Sicht untersucht und daraus resultierende Fehlschlüsse und Fehlassoziationen aufgezeigt $(\S 1)$; sodann wird ausführlich dargelegt, was eine Künstliche Intelligenz im technischen Sinne ist, was sie zu leisten vermag, aber auch was sie nicht ist. Obligatorisch hierfür ist auch eine gewisse Betrachtung allgemeiner Prinzipien der Informatik und der daraus resultierenden Grenzen. Hierbei wird auch untersucht, ob das Ergebnis einer Künstlichen Intelligenz vorhergesagt werden kann, also ob sich eine Künstliche Intelligenz deterministisch verhält, ${ }^{25}$ was das Sollverhalten einer Künstlichen Intelligenz darstellt, was unter einem Fehler bei Künstlicher Intelligenz zu verstehen und welches Verhalten generell vorhersehbar ist. Im Rahmen dessen soll dargelegt werden, was ihr Verhalten ausmacht, ob ein Wille besteht und was eine Künstliche Intelligenz überhaupt wissen kann (§ 2). Darauf aufbauend werden die zivilrechtlichen Auswirkungen im Vertrags- und Deliktsrecht untersucht, wobei nach wie vor streitig ist, ob durch Künstliche Intelligenz wirklich neue Probleme im Zivilrecht entstehen. ${ }^{26}$ Unter Einbeziehung der Ergebnisse der vorherigen Teile sollen die Auswirkung der Autonomie und einer möglichen (Un-)Vorhersehbarkeit von Künstlicher Intelligenz dargelegt werden. Dies soll im Vergleich zu herkömmlichen technischen Einrichtungen anhand der Zurechnung von Willenserklärungen, Verhalten und Wissen geschehen. (§ 3).

25 Was teilweise bestritten wird, vgl. Dettling/Krüger, PharmaR 2018, 513, 514; Pieper, InTeR 2018, 9, 11; Riehm, ITRB 2014, 113; Spindler, CR 2015, 766; Söbbing/Spindler, S. V; eingeschränkt wohl auch Denga, CR 2018, 69, 70; Kirn/ Müller-Hengstenberg, MMR 2014, 225, 228.

26 Teilweise besteht die Ansicht, dass das geltende Recht bereits hinreichend diesem Phänomen und den damit verbundenen Besonderheiten begegnet, vgl. MünchKommBGB/Säcker, Einl. BGB Rn. 190; MünchKommBGB/Busche, Vor $§ 145$ BGB Rn. 37 ff. 


\section{$\S 1$ Begriff und Übersetzungsproblem}

Ausgangspunkt für die Betrachtung ist der Begriff »Künstliche Intelligenz«. Bei diesem Begriff stellt sich schon die Frage, ob mit diesem bereits eine Erwartungshaltung an die Entität verbunden ist, die durch ihn benannt wird. Hierzu gehört auch die Frage, ob die Übersetzung des ursprünglich englischsprachigen Begriffs »artificial intelligence» in der deutschen Sprache die gleiche Bedeutung hat und damit beschrieben werden kann, was »Künstliche Intelligenz« (im technischen Sinne) eigentlich ist. Gerade der Bestandteil »Intelligenz« kann aufgrund seiner unscharfen Bedeutung problematisch sein. ${ }^{27} \mathrm{Um}$ dem näher zu kommen, ist es hilfreich, sich den Ursprung des Begriffs darzulegen. Dies ist indes keine sprachliche Haarspalterei, sondern notwendige Voraussetzung, um die (öffentliche) Debatte über Künstliche Intelligenz nachvollziehen und Fehlentwicklungen erkennen zu können. ${ }^{28}$ Der Begriff »artificial intelligence« wurde von McCarthy et al. in einem Aufruf im Jahre 1955 geprägt. ${ }^{29}$ Ein in der Literatur vorgeschlagener und weniger problematische - da ohne den Begriff »intelligence« auskommender - Alternativbegriff wäre »computational rationality ${ }^{30}$

Zerlegen lässt sich der Begriff »artificial intelligence« in die beiden Bestandteile »artificial« und »intelligence«. »Artificial« kann im Deutschen mit »künstlich « übersetzt werden, ${ }^{31}$ stammt aber vom lateinischen Wort »artificialis $\ll^{32} \mathrm{ab}$, welches ursprünglich für »kunstfertig« oder $»$ kunstgerecht« steht. ${ }^{33}$ Dieses »kunstgerechte«, welches auf das lateinische Wort »ars« zurückgeht, schwingt im deutschen Wort »künstlich« nicht mehr mit. ${ }^{34}$ Dennoch kann man auch mit der engeren Bedeutung des Begriffs in der deutschen Sprache unter »künstlich« alles subsumieren, was nicht naturgeschaffen ist. ${ }^{35}$ In diesem Kontext ist das Wort »künstlich« in

27 Ebenso Herberger, NJW 2018, 2825, 2826; Konertz/Schönhof, ZGE/IPJ 10 (2018), 379, 380; Söbbing, InTeR 2018, 64; Grapentin, S. 63 f.

28 Im Ergebnis ebenso Herberger, NJW 2018, 2825, 2826.

29 Deutschsprachige Übersetzung zu finden bei Russell/Norvig, S. 40.

30 Russell/Norvig ${ }^{2}$, S. 17.

31 Langenscheidt Großwörterbuch Englisch, S. 40 (»artificial«).

32 The Oxford English Dictionary, Vol. I, S. 666, (»artificial«).

33 Der neue Georges, S. 463 (»artificialis«).

34 Kritisch hierzu Herberger, NJW 2018, 2825, 2827.

35 Kluge, S. 547 (»Kunst/künstlich«). 
$»$ Künstlicher Intelligenz« insoweit eine treffende Übersetzung für eine nicht naturgeschaffene Entität.

Das Wort »intelligence« stammt vom lateinischen Begriff »intelligentia $\ll,{ }^{36}$ was im Deutschen mit $»$ Vorstellung $«, ~ »$ Idee $«, ~ »$ Begriff $«, ~ »$ Einsicht «, »Erkenntnis« oder »Verständnis« übersetzt werden kann. ${ }^{37}$ In der deutschen Sprache wird das Wort »Intelligenz« verwendet, welches allerdings nicht direkt von »intelligentia « abstammt, sondern vom Wort »intellegere « oder »intellectum «, ${ }^{38}$ was die Bedeutung »wahrnehmen«, »merken«, »erkennen« oder »empfinden « hat. ${ }^{39}$ Es stammt also von einem Verb $a b$ und nicht von einem Substantiv. Allerdings darf das nicht überbewertet werden, da anzunehmen ist, dass beide Worte wieder auf den lateinischen Begriff »intelligentia« zurückführbar sind, ${ }^{40}$ sodass der nicht weiter vertiefte etymologische Ansatz wohl wenig Erkenntnisgewinn mit sich bringt.

Aussichtsreicher ist, welche Bedeutung den Worten »intelligence« und »Intelligenz« in der deutschen und englischen Sprache in der Gegenwart zukommt. In der deutschen Sprache klingt mit dem Begriff »Intelligenz« immer etwas »Schöpferisches« oder »Kritisches«, also etwas Intellektuelles mit, was im Englischen nicht der Fall ist. Dort existiert der zusätzliche Begriff »intellectuals«, sodass der deutsche Begriff »Intelligenz« im Gegensatz zum englischen Begriff »intelligence« ein wertender ist. ${ }^{41}$ Auch hat sich der Begriff in der englischen Sprache anders entwickelt als in der deutschen Sprache. Dem Begriff »intelligence« kommt noch eine weitere Bedeutung zu, die anderen Sprachen fremd ist. Gerade im militärischen Umfeld ist das Wort auch mit »Spionage «, »Geheimdienst« oder auch einfach nur $»$ Information ${ }^{42}{ }^{2}$ übersetzbar. Gemeint ist, dass eine intelligence Informationen gewinnt, die essenziell für Entscheidungen und Aktionen sind..$^{43}$ Legt man dieses Verständnis zugrunde, so wäre die Intelligenz in der Künstlichen Intelligenz abseits der psychologischen, philosophischen oder soziologischen Betrachtung des Intelligenzbegriffs nur ein (künstliches) Mittel zur Informationsgewinnung. Allerdings darf dies nicht darüber hinwegtäuschen, dass der Begriff »Künstliche Intelligenz« keine allge-

36 The Oxford English Dictionary, Vol. VII, S. 1069 (»intelligence«).

37 Der neue Georges, S. 2655 (»Intelligentia«).

38 Kluge, S. 444 (»intelligent $\ll)$.

39 Der neue Georges, S. 2655 f. (»Intellectus/intellego«).

$40 \quad I m$, S. $9 \mathrm{ff}$.

41 Im, S. 127.

42 Langenscheidt Großwörterbuch Englisch, S. 423 (»intelligence«).

43 Im, S.115 ff. 
meine Beschreibung von Entitäten ist, vielmehr mahnt die begriffliche Auseinandersetzung zur Vorsicht, daraus gerade keine Überinterpretation eines Phänomens zu folgern. Aufgrund der Wertung des Begriffs »Intelligenz« und der Bedeutung des Wortes »künstlich«, kann auch der Begriff »gekünstelte Intelligenz« als Übersetzung verwendet werden. ${ }^{44}$ Bleibt man beim Ursprungsbegriff, muss dieser als wertfreier Eigenname für eine Klasse von verschiedenen (technischen) Entitäten betrachtet werden. Diese sollen im Rahmen der weiteren folgenden Untersuchung näher dargelegt werden.

44 Lämmel/Cleve, S. 11. 


\section{$\S 2$ Wesen: Willen, Wissen und Verhalten}

Da der Begriff der Künstlichen Intelligenz problembehaftet ist und zu Fehlassoziationen verleitet, selbst aber kaum einen Aussagegehalt über die beschriebene Entität hat, ist zunächst das Wesen einer Künstlichen Intelligenz genauer zu untersuchen. Von besonderem Interesse sind Fragen nach einem möglichen Willen, möglichem Wissen und dem Verhalten. Hierbei ist eine gewisse technische Vertiefung unumgänglich. ${ }^{45}$ Das Forschungsgebiet in der Informatik, das sich mit dem beschäftigt, was heute allgemein »Künstliche Intelligenz« genannt wird, ist sogar älter als ihr Name selbst; schon seit 1943 und damit seit Aufkommen des programmierbaren Rechners ${ }^{46}$ wird in dieser Disziplin geforscht. ${ }^{47}$ In diesem Kontext kann zudem davon ausgegangen werden, dass sich Künstliche Intelligenz auf Maschinen ${ }^{48}$ im Speziellen auf programmierbare Maschinen, also Computer bezieht. Um die Grundfrage nach den möglichen Unterschieden zu anderen Computerprogrammen bzw. -software ${ }^{49}$ genauer zu untersuchen, ist zunächst weiter auszuholen. $\mathrm{Zu}$ Beginn soll daher auf die Problematik des Vergleichs von Computern und Menschen eingegangen werden (A.), sodann werden kurz mögliche Definitionsversuche und deren Schwierigkeiten dargelegt (B.). Nachfolgend wird eine Reihe von gängigen KI-Methoden vorgestellt und am Beispiel des künstlichen neuronalen Netzes vertieft (C.). Danach muss für ein weiteres Verständnis dargelegt werden, was eigentlich ein Algorithmus ist und wie der Weg vom Modell zur Computersoftware aussieht (D.). Sodann wird der Determinismusbegriff in der Philosophie und der Informatik vorgestellt, um die rechtstatsächliche Ausgangsfrage des Determinismus zu klären (E.). Im Folgenden stellt sich dann die Frage, wie ein Fehler einer Künstlichen Intelligenz zu klassifizieren ist, welches Verhalten noch vorhersehbar ist und ob es Ansätze einer (teilweisen) Erklärbarkeit gibt (F.). Abschließend bleibt noch zu klären, was Wissen ist und was eine Künstliche Intelligenz wissen kann (G.).

45 Ebenso Herberger, NJW 2018, 2825.

46 Durch das sog. von Neumann-Modell, vgl. v. Neumann, IEEE Annals of the History of Computing 15 (1993), Issue 4, 27 ff.

47 McCulloch/Pitts, Bulletin of Mathematical Biophysics 5 (1943), $115 \mathrm{ff}$.

48 Also nicht auf künstlich geschaffenes biologisches Material.

49 Im Weiteren wird davon ausgegangen, dass die Begriffe »Computerprogramm«, »Programm« und »Software« - unabhängig von möglichen inhaltlichen Unterschieden - Synonyme sind. 


\section{A. Problematik des Vergleichs zum Menschen}

Das Phänomen der Künstlichen Intelligenz ist Gegenstand verschiedener Disziplinen. Unter anderem wird es auch philosophisch betrachtet. ${ }^{50}$ Hier befasst sich die Teildisziplin der Ethik mit Fragestellungen, wie auf gewisse Phänomene aus moralischer Sicht zu reagieren ist bzw. wäre und wie Entitäten im moralischen Universum einzuordnen sind bzw. wären. ${ }^{51}$ Weiterhin wird aber auch ein Vergleich zwischen zwei Architekturen durchgeführt: Dem Menschen und dem Computer bzw. der Maschine. ${ }^{52}$ Ein solcher Vergleich kann durchaus sinnvoll sein, allerdings verleitet er auch dazu, die Eigenschaften einer Architektur auf die andere zu übertragen. Einerseits kann damit die Angst verbunden sein, dass Maschinen dem Menschen überlegen sein können; solche Gedanken findet man gewiss schon in Hobbes' Leviathan, in der Gestalt des vom Menschen geschaffenen »künstlichen Tiers «.$^{53}$ Anderseits neigt man dazu, die Architekturen nur noch im Vergleich zueinander zu sehen, ohne deren spezifische Fähigkeiten zu berücksichtigen. In Bezug auf Künstliche Intelligenz heißt dies, in dieser etwas Intellektuelles oder gar Menschenähnliches zu sehen, dessen Ziel es ist, wie der Mensch zu denken ${ }^{54}$ oder diesem sogar darin überlegen zu sein. ${ }^{55}$

Zunächst stellt sich erstens die Frage, ob die Probleme, die der Mensch lösen kann, auch durch einen Computer gelöst werden können. Die Beantwortung dieser Frage beginnt bei der nicht bewiesenen Church-TuringThese. Diese sagt aus, dass die (hypothetische) Turing-Maschine ${ }^{56}$ alle intuitiv berechenbaren Funktionen berechnen kann. ${ }^{57}$ Vereinfacht wird weiterhin angenommen, dass ein Computer eine reale Umsetzung einer

50 Vgl. Russell/Norvig, S. 26 ff.

51 Vgl. Loh, InTeR 2017, 220.

52 Russell/Norvig, S. 1176.

53 Hobbes, S. 6.

54 Dettling/Krüger, PharmaR 2018, 513, 514.

55 So aber wohl Reichwald/Pfisterer, CR 2016, 208, 211.

56 Eine Turing-Maschine kann an dieser Stelle als hypothetisches und nicht real umsetzbares Modell eines (universellen) Computers gesehen werden, ist aber eigentlich ein formales Modell, welches für exakte, mathematische Aussagen bei der Berechenbarkeit verwendet wird, wobei mehrere Arten der Turing-Maschine mathematisch modelliert werden können. Weder der formale Berechenbarkeitsbegriff noch die formalen Definitionen der möglichen Arten der Turing-Maschine sollen an dieser Stelle weiter erörtert werden, da sie für das Verständnis der Argumentation nicht von Bedeutung sind; vgl. vertiefend Hoffmann, Theoretische Informatik, S. $254 \mathrm{ff}, 277 \mathrm{ff}$.

57 Kleene, S. 376. 
Turing-Maschine $\operatorname{sei}^{58}$ und dass unter dem (nicht wohldefinierbaren) Begriff »intuitiv berechenbar« alle (mathematischen) Funktionen verstanden werden, die man - also auch der Mensch - überhaupt berechnen kann. ${ }^{59}$ Daraus würde folgen, dass ein Computer alles berechnen könnte, was berechenbar ist, also für alle berechenbaren Probleme eine mögliche mächtigste Problemlöse-Instanz sei. Daraus folgt aber nicht, dass auch alle berechenbaren Probleme jemals durch einen Computer berechnet werden. Für jedes Problem ist es notwendig, den Computer durch Computersoftware, die wiederum auf Algorithmen basieren, ${ }^{60}$ die Berechnung durchführen zu lassen, sofern dies praktisch umsetzbar ist. Zugleich können (alle) berechenbaren Probleme (theoretisch) auch durch den Menschen gelöst werden; er ist daher auch eine mögliche, mächtigste Problemlöse-Instanz (für berechenbare Probleme). Gewiss lösen auch Menschen diese Probleme algorithmisch, dennoch darf daraus nicht geschlossen werden, dass die Klasse der Probleme, die Mensch und Maschine lösen können, äquivalent sei. ${ }^{61}$ Dies ist ein prädikatenlogischer Fehlschluss, da ein Mensch nicht nur berechenbare Probleme lösen bzw. versuchen kann zu lösen, sondern auch gänzlich andere, ${ }^{62}$ beispielsweise theologische oder moralische Probleme. ${ }^{63}$ Daraus folgt weiter, dass Künstliche Intelligenz als Computersoftware auf berechenbare Probleme beschränkt ist, was der Mensch nicht ist.

Zweitens stellt sich die damit verbundene Frage nach der Bedeutung der Hypothese der »starken Künstlichen Intelligenz«. ${ }^{64}$ Während »schwache Künstliche Intelligenz« nur eine einzelne Aufgabe besser lösen soll als der Mensch, soll »starke Künstliche Intelligenz« eine generelle Problemlösungsmaschine für lösbare Probleme darstellen und damit eine Maschine sein, die denkt. ${ }^{65}$ Dieser Unterschied wird teilweise sogar als Meinungsstreit zweier Thesen dargestellt. ${ }^{66}$ Zumindest in der technischen Betrach-

58 Was genau genommen unzutreffend ist, da eine Turing-Maschine ein unendliches Band (als Speicher) besitzt, was jedoch in der Realität eines begrenzten Universums nicht konstruierbar ist.

59 Hoffmann, Theoretische Informatik, S. 308.

60 S. u., Vom Modell über den Algorithmus zur Computersoftware (S. 52).

61 So aber Reichwald/Pfisterer, CR 2016, 208, 209.

62 Gilt unter anderem auch für nicht-berechenbare Probleme.

63 Dies gilt nur soweit, solange kein Modell gefunden wird, dass die Moral mathematisch abbildet.

64 Zu finden bei Keßler, MMR 2017, 589, 592; Dettling/Krüger, PharmaR 2018, 513, 515.

65 Dettling/Krüger, PharmaR 2018, 513, 514.

66 Pieper, InTeR 2018, 9, 11. 
tungsweise spielt die starke Künstliche Intelligenz aber keine ernst zu nehmende Rolle. Diejenigen, die sich mit der technischen Umsetzung von Künstlicher Intelligenz befassen, beachten sie schlichtweg nicht oder nur peripher. ${ }^{67}$ Gewiss ist schwer darzulegen, dass etwas nicht existiert. ${ }^{68}$ Nach heutigem Kenntnisstand bestehen jedoch noch keine Konzepte, geschweige denn Applikationen einer starken Künstlichen Intelligenz. ${ }^{69}$ Zudem reicht ein Blick in die technische Literatur, um zu verstehen, dass kein »künstlicher Wille« im Sinne des menschlichen Willens, »künstliches Bewusstsein« oder »künstliche Emotionen « geschaffen werden sollen, ${ }^{70}$ sondern Fragen der Aussagen- und Prädikatenlogik, des stochastischen Schließens und heuristischer Suchverfahren behandelt werden. ${ }^{71}$ Es geht also - wie noch dargelegt wird - um viel konkretere Ansätze zur Lösung bestimmter Probleme und nicht um einen künstlichen Menschen (sog. »Homunculus $\ll)^{72}$

Letztendlich ist es notwendig, zwischen »Verhalten«, »Handeln« und »Denken« zu unterscheiden. »Denken« ist ein innerer Prozess, der von außen nicht betrachtet werden kann. ${ }^{73}{ }$ Verhalten $«$ ist dagegen ein Prozess einer verkörperten Entität über die Zeit verbunden mit einer von außen beobachtbaren Zustandsänderung, ohne dass es auf die Begründung dieser Zustandsänderung ankommt. ${ }^{74}$ Bei einer Handlung kommt zum Verhalten noch hinzu, dass sie zielgerichtet ist. ${ }^{75}$ Dies ist zwar ebenfalls ein inneres Attribut, jedoch im Hinblick ihres Zwecks zeigt eine Künstliche Intelligenz Verhalten. Durch die Beobachtung von außen kann aber nur auf das allgemeine Verhalten bzw. Handeln, nicht aber auf dessen Begründung beispielsweise im Sinne von intelligentem Handeln - oder gar auf einen Denkprozess geschlossen werden. Nur weil eine Entität eine (richtige) Lösung (eines berechenbaren Problems) findet, heißt dies nicht, dass sie

67 Russell/Norvig, S. 1176.

68 Man müsste für alle (real existierenden) Entitäten zeigen, dass sie etwas nicht sind, was ein klassisches Induktionsproblem ist, vgl. Mittelstraß/Haas: Induktion, Bd. 3, S. $594 \mathrm{ff}$.

69 Buxmann/Schmidt, S. 6.

70 Vgl. allgemein Ertel; Jarosch und Pérez Castaño.

71 Vgl. insbesondere Ertel, S. 25 ff.

72 Russell/Norvig, S. 1176.

73 Mittelstraß/Mittelstraß/Lorenz: Denken, Bd. 2, S. 154.

74 Sandkühler/Regenbogen: Verhalten, Bd. 3, S. 2882 f.

75 Streitig, da bei einer intentionalistischen Ansicht das Wollen hinzukommt, vgl. Sandkühler/Lumer: Verhalten, Bd. 1, S. 2882 f.; im Folgenden wird aber von einer nicht-intentionalistischen Handlung ausgegangen, die sich nur durch die Zielgerichtetheit unterscheidet. 
auch sinnvoll gefunden wurde oder gar ein Verständnis über den Lösungsweg vorliegt. Auf die inneren Zustände kann nicht geschlossen werden. Dies zeigt das Gedankenexperiment des sog. »Chinesischen Zimmers «. ${ }^{76}$ Hierbei sitzt eine Person, die der chinesischen Sprache nicht mächtig ist, in einem Raum und erhält Karten mit chinesischen Schriftzeichen. Aufgabe der Person ist es, mithilfe eines für sie verständlichen und vollständigen Buches, für alle denkbaren ankommenden Schriftzeichen eine Antwort, die wiederum in Chinesisch zu verfassen ist, aufzuschreiben und herauszugeben. In diesem Sinne erzeugt das chinesische Zimmer immer eine korrekte Antwort. Dabei ist für außenstehende Beobachter - die das Innere des Zimmers nicht kennen - nicht bekannt, ob die Person im Inneren (wirklich) versteht, was sie macht. Damit kann sie nur das Verhalten dieser Entität betrachten. ${ }^{77}$ Ein anderer Ansatz besteht darin, einfach alle möglichen Lösungswege durchzuprobieren, bis ein korrektes Ergebnis vorliegt; hierfür ist es nur notwendig zu wissen, wie das korrekte Ergebnis aussieht (sog. »Brute-Force-Ansatz $\ll) .{ }^{78}$ In diesem Sinne hat auch der im juristischen Schrifttum gerne zitierte »Turing-Test« nicht das Ziel, zu beweisen, dass ein Computer denkt, sondern nur, dass sich ein Computer wie ein Mensch (nach außen) verhält. ${ }^{79}$ Die Frage, ob Computer denken können oder zumindest vorspielen können, zu denken, ist unerheblich. Der niederländische Informatiker Edsger Dijkstra hat es mit dem Zitat »Die Frage, ob Maschinen [wie Menschen] denken können, ist genauso interessant wie die Frage, ob U-Boote [wie Menschen] schwimmen können «, ${ }^{80}$ polemisch auf den Punkt gebracht. Künstliche Intelligenz hat somit nicht das Ziel, Computer wie Menschen denken zu lassen, ${ }^{81}$ sondern nur, sie wie Menschen handeln zu lassen. ${ }^{82}$ Darüber hinaus sei erwähnt, dass an Maschinen, welche den gerne zitierten Turing-Test ${ }^{83}$ erfüllen sollen, kaum geforscht wird ${ }^{84}$ und dieser Test daher keine große Relevanz in der KI-Forschung hat. ${ }^{85}$

$76 \mathrm{Zu}$ finden auch bei Schönberger, ZGE/IPJ 10 (2018), 35, 40.

77 Searle, The Behavioral and Brain Science 3 (1980), $417 \mathrm{ff}$.

78 Russell/Norvig, S. 45.

79 Russell/Norvig, S. 23 f.

80 Mündliches Zitat, ACM 1984 South Central Regional Conference. Zu beachten ist, dass U-Boote im Englischen nicht schwimmen, sondern sich durch das Wasser bewegen (»move through the water«), vgl. Russell/Norvig ${ }^{2}$, S. 948.

81 Ebenso Stiemerling, CR 2015, 762, 765.

82 Russell/Norvig, S. 1178.

83 Ménière/Pihlajamaa, GRUR 2019, 332, 333; Söbbing, InTeR 2018, 64; v. Graevenitz, ZRP 2018, 238, 240.

84 Russell/Norvig, S. 24.

85 Ertel, S. 4. 
Somit ist auch der Rahmen dafür gesteckt, wie das Phänomen der Künstlichen Intelligenz juristisch betrachtet werden muss. Es hilft nicht, sich mit reinen Gedankenexperimenten zu beschäftigen.$^{86}$ Um der Steuerungs- und Kontrollfunktion der Rechtswissenschaften gerecht zu werden, ${ }^{87}$ sind daher nur rechtstatsächliche Phänomene von Relevanz, die technisch möglich sind oder bei denen es zumindest signifikant wahrscheinlich ist, dass sie in näherer Zukunft umgesetzt werden. Insofern betrachtet die Rechtswissenschaft hier bestehende oder aufkommende Konflikte. ${ }^{88}$ Technisch - und damit auch juristisch - relevant ist nur der Bereich, der als »schwache Künstliche Intelligenz« bezeichnet wird. ${ }^{89}$ Sich juristisch derzeit mit der »starken Künstlichen Intelligenz « zu beschäftigen, wäre genauso, als würde man sich mit dem Recht der erdähnlichen Exoplaneten beschäftigen. Insofern wird im Folgenden unter »Künstlicher Intelligenz« nur noch die »schwache Künstliche Intelligenz« verstanden.

\section{B. Definitionsversuche}

Nach den bisherigen Ausführungen steht bislang nur fest, was Künstliche Intelligenz nicht ist. Zwar existiert aus technischer Sicht keine umfassend anerkannte Definition, ${ }^{90}$ allerdings finden sich durchaus allgemeine Beschreibungen, die das Phänomen der Künstlichen Intelligenz erörtern. Wie dargelegt geht es dabei nur um Handlungen, die auch vom Menschen ausgeführt werden können. Ein treffender Definitionsversuch stammt von Rich: »Künstliche Intelligenz ist die Forschung, wie man Computer Dinge ausführen lassen kann, die zur Zeit noch vom Menschen besser beherrscht werden $\ll .{ }^{91}$ Vom Wortlaut her problematisch, aber auch von Rich erkannt, ist, dass sobald ein Computer eine Tätigkeit besser kann als der Mensch,

86 Die Verf. können natürlich nicht ausschließen, dass derartige Technologien in $\mathrm{Zu}-$ kunft entstehen, halten sie aber derzeit für unwahrscheinlich.

87 Vgl. Kaufmann/Hassemer/Neumann/Neumann, S. $396 \mathrm{ff}$.

88 Vgl. Steiniger, NJW 2015, 1072, $1073 \mathrm{f}$.

89 Anders Pieper, InTeR 2018, 9, $14 \mathrm{f}$.

90 Ebenso im juristischen Schrifttum, Pieper, InTeR 2018, 9, 11; Söbbing, InTeR 2018, 64; Hoeren/Niehoff, RW 2018, 47, 49; im technischen Schriftum: Ertel, S. 1 ff.; Rich, S. 1; Russell/Norvig, S. 22 ff.

91 Rich, S. 1. Dieses Zitat bezieht sich auf die Handlung, nicht auf das Denken, wie von Reichwald/Pfisterer, CR 2016, 208, 211 behauptet. Vgl. auch die englische Sprachfassung, welche von »[...] make computer do things [...], people better do« spricht, Rich/Knight, S. 3. 
diese Lösung keine Künstliche Intelligenz mehr wäre. ${ }^{92}$ Damit ist dann einerseits mehr das Ziel der aktuellen KI-Forschung gemeint, andererseits wird klar, dass KI-Methoden irgendwann zur regulären Informatik werden. ${ }^{93}$ Zudem konnte ein Computer immer schon gewisse Dinge besser als der Mensch; der Computer wurde ursprünglich als Rechenmaschine geschaffen, sollte also zumindest das Rechnen als Handlung immer schon besser - in diesem Sinne schneller - können als der Mensch. ${ }^{94}$ Würde man das Ganze auf die Spitze treiben, würde dies sogar auf jede Maschine zutreffen, die sich der Mensch als Hilfsmittel heranzieht.

Nimmt man neben dem Begriff des Computers den Begriff des Agenten hinzu, wird eine andere Sichtweise eröffnet. Agenten sind Entitäten, die ihre Umgebung wahrnehmen und entsprechend reagieren. ${ }^{95} \mathrm{Zwar}$ ist auch jeder Computer ein Agent, allerdings wird er nun von seinem Verhalten und seiner Interaktion her betrachtet. Künstliche Intelligenz kann man nun als den Versuch sehen, den besten rational handelnden Agenten auf einer bestimmten Architektur für ein bestimmtes Problem zu finden. ${ }^{96}$ Gewiss ist damit nicht definiert, was man unter »rationalem Handeln« versteht, dies kann aber auch offenbleiben. Deutlich wird aber, dass es um die Lösung bestimmter (berechenbarer) Probleme mit einem bestimmten - dem möglichst besten - Ansatz geht, so dass dem Agenten scheinbar eine Form von Intelligenz zukommt. Folglich ist unter Künstlicher Intelligenz Computersoftware zu verstehen, welches zur verbesserten Lösung von bestimmten Problemen eingesetzt wird. ${ }^{97}$ Die Fähigkeiten derartiger Computersoftware sind Folge einer fortschreitenden Entwicklung der Leistungsfähigkeit von Computern, mit der nun bestehenden Möglichkeit, schnell auf große Datenmengen zuzugreifen und diese systematisch zu analysieren. ${ }^{98}$

92 In diesem Sinne Rich, S. 1.

93 Zitat von Kohlhase aus der Vorlesung zur Künstlichen Intelligenz v. 19.10.2017.

94 Aspray/Aspray, S. $251 \mathrm{ff}$.

95 Zu eng daher bei Sester/Nitschke, CR 2004, 548.

96 Russell/Norvig, S. 25 f.

97 So schon bei Konertz/Schönhof, ZGE/IPJ 10 (2018), 379, $380 \mathrm{ff}$.

98 Russell/Norvig, S. 36 f. 


\section{Technologien der Künstlichen Intelligenz}

Die bisherigen Beschreibungen waren freilich noch abstrakt. Genau wie es auch bei dem eingangs genannten technischen Phänomen der Eisenbahn notwendig ist, sich mit dessen konkreten technischen Eigenarten zu befassen, um aus diesen rechtswissenschaftlich fundierte Schlussfolgerungen $\mathrm{zu}$ ziehen, ist es unumgänglich, sich mit den Funktionsprinzipien realer KI-Methoden auseinanderzusetzen, insbesondere, um deren Wesen näher zu ergründen.

\section{Vom einfachen Schachcomputer zu AlphaZero}

Bevor jedoch ausgewählte KI-Methoden vorgestellt werden, sei zu Beginn an einem einfachen Beispiel die reale Verwendung von Künstlicher Intelligenz sowie deren Fähigkeiten dargelegt. Als prägendes Beispiel werden auch in der juristischen Literatur gerne die Leistungen bei Brettspielen von Schach oder GO herangezogen. ${ }^{99}$ In den letzten Jahren sind Brettspielcomputer mitunter in ihrer Architektur bemerkenswerten Veränderungen unterzogen worden. Das Ziel ist nicht nur, Programme zu erschaffen, die besser als ein Mensch spielen, sondern auch außerordentlich komplexe Probleme effizienter zu bewältigen. Das Brettspiel Schach kann auf viele Weisen eröffnet werden: Insgesamt stehen einem Spieler 20 Möglichkeiten offen, ein Spiel zu eröffnen. ${ }^{100}$ Ebenso existieren 20 weitere Möglichkeiten des Gegners, sein Spiel zu eröffnen. So sind nach der ersten Runde bereits 400 verschiedene Stellungen möglich. Die Menge aller möglichen Schachspiele wird mit $10^{120}$ beziffert. ${ }^{101}$ Im Vergleich sei angemerkt, dass nur ca. $10^{79}$ Teilchen im bekannten Universum vermutet werden. ${ }^{102}$ Als Folge würde ein Brute-Force-Ansatz schon an der Komplexität des Problems scheitern. Die ersten Schach-Algorithmen haben versucht, alle möglichen Züge für möglichst viele Runden zu simulieren und deren Effektivität zu bewerten. ${ }^{103}$ Selbst mit erheblicher Rechenleistung konnten nur einige wenige Runden vorberechnet werden. Nachfolgesysteme hatten primär das Ziel, diesen Algorithmus effizienter zu betreiben, beispielsweise indem der Anteil an offensichtlich erfolglosen Zügen frühzeitig erkannt und ignoriert

99 Grapentin, NJW 2019, 181, 183; Pieper, InTeR 2018, 9, $11 \mathrm{f}$.

100 Die acht Bauern und zwei Springer können jeweils zwei Felder erreichen.

101 Shannon, Philosophical Magazine, Ser. 7 (1950), Vol. 41, 256, 259 f.

102 Hanslmeier, S. 520.

103 Vgl. Shannon, Philosophical Magazine, Ser. 7 (1950), Vol. 41, 256, 262 ff. 
wurde. ${ }^{104}$ Die gesparte Rechenleistung konnte in die Evaluation vielversprechenderer Züge reinvestiert werden. Dies findet sich auch in modernen Schach-KIs, wie Stockfish, wieder. ${ }^{105}$

Einen Paradigmenwechsel erfuhren die Spielecomputer spätestens ${ }^{106}$ mit dem Programm AlphaZero für Brettspiele wie Schach, Shogi und GO, welche die Suchstrategien durch künstliche neuronale Netze ${ }^{107}$ und MonteCarlo-Suchbäume verdrängten. ${ }^{108}$ Das Training dieser künstlichen neuronalen Netze erfolgte jedoch ausschließlich durch Partien gegen sich selbst. Vereinfacht lässt es sich wie folgt veranschaulichen: Zu Beginn stehen sich zwei (virtuelle) Teams gegenüber, ${ }^{109}$ welche noch nie GO gespielt haben. Sie wissen nichts über das Spiel, nicht einmal, wann ein Sieg vorliegt, wurden aber auf einen Sieg hin optimiert. Ein Teammitglied (das neuronale Netz) übernimmt die Rolle des Strategen und ermittelt, welche Züge infrage kommen, das andere Teammitglied (das Suchprogramm) übernimmt die Rolle des Überwachenden und versucht, zu bestimmen, wie gut diese Züge tatsächlich waren. Abwechselnd generieren die Teams nun ihre Strategien, um die Figuren auf dem Brett zu verschieben. Ein »virtueller Schiedsrichter« achtet auf die Einhaltung der Regeln und bestimmt, wann eine Partie gewonnen wurde oder die zulässige Spielzeit abgelaufen ist. Sobald die Partie entschieden oder beendet wurde, versucht das neuronale Netz seine Taktik anzupassen, indem es das Feedback des Suchalgorithmus und das des Schiedsrichters berücksichtigt. Im Anschluss beginnt die nächste Partie. Am Ende des Trainings können die Strategen die erfolgversprechendsten potentiellen Spielzüge ausfindig machen, sodass der Suchalgorithmus nur die besten Strategien auswerten muss. ${ }^{110}$ Alleine dieses Beispiel zeigt bereits, worum es bei Künstlicher Intelligenz geht: Es wurde versucht, den am besten rational handelnden Agenten für die Spiele zu finden, nicht jedoch die Spiele wie ein Mensch »denkend« zu lösen.

104 Vgl. Nilsson, S. 89 f.

105 Czarnul, in: Shi et al., S. 458.

106 Self-play Neuronale Netze wurden bei simpleren Spielen schon früher in der Literatur vorgeschlagen, vgl. für das Spiel Backgammon Tesauro, Artificial Intelligence 134 (2002), $181 \mathrm{ff}$.

107 S. u., Beispiel: (Künstliches) neuronales Netz (S. 45).

108 Silver/Hubert/Schrittwieser et al., S. 12.

109 In Wirklichkeit spielt der Computer gegen sich selbst.

110 Silver/Schrittwieser/Simonyan et al. Nature, Vol. 550 (2017), $354 \mathrm{f}$. 
II. Methoden der Künstlichen Intelligenz im Überblick

Im vorherigen Abschnitt wurden bereits zwei KI-Methoden genannt: »Das künstliche neuronale Netz« und »Suchstrategien«. Schon daran zeigt sich, dass es eine Fehlvorstellung ist, dass es die Künstliche Intelligenz »an sich« gibt. Unter den Begriff »Künstliche Intelligenz« fallen vielmehr eine Fülle von unterschiedlichen Ansätzen und Verfahren, welche in der Lage sind, bestimmte Probleme zu lösen. Nachfolgend sollen eine Reihe von bekannten Methoden der Künstlichen Intelligenz und ihre Anwendungsgebiete in einer kurzen Übersicht charakterisiert werden. Hierbei soll auch die Unterscheidung zwischen Künstlicher Intelligenz und der Untergruppe des sog. »Machine Learning « ${ }^{111}$ illustriert werden. ${ }^{12}$

\section{Suchstrategien}

$\mathrm{Zu}$ den wohl einfachsten und ältesten Formen der Künstlichen Intelligenz zählen die Suchstrategien. Sie eignen sich zum Lösen einer Vielzahl von Problemen des Alltags. Sie finden bei heuristischen Suchen Anwendung, aber auch - in stark optimierter Form - bei Routenplanern in der Logistik. ${ }^{113}$ Anschauliche Beispiele für die Suchstrategien sind die Ermittlung eines Weges durch ein Labyrinth oder die Lösung eines Schachspiels. ${ }^{114} \mathrm{Ob}-$ gleich das Durchschreiten eines Labyrinths und das Lösen eines Schachspiels anscheinend wenig gemeinsam haben, können sie dennoch auf ein gemeinsames Problem reduziert werden: Wie soll sich der Algorithmus an einer beliebigen Stelle im Labyrinth bzw. zu einer beliebigen Spielsituation während des Schachspiels entscheiden? Während das Labyrinth-Problem recht übersichtlich ist, da hier immer nur die Wahl zwischen rechts oder links besteht, ist die Wahl beim Schach vielfältiger. Der Algorithmus kann mit jeder Figur eine bestimmte Menge an Spielzügen machen. Eine Suchstrategie würde nun sämtliche Entscheidungsmöglichkeiten ausprobieren;

111 Auf Deutsch »Maschinelles Lernen«.

112 Das sog. »künstliche neuronale Netz« wird im nachfolgenden Abschnitt vertiefend erläutert, s. u., Beispiel: (Künstliches) neuronales Netz (S. 45).

113 Beispielsweise der sog. »Dijkstra Algorithmus«, vgl. Nha/Djahel/Murphy, S. 1 ff.; Cormen/Leiserson/Rivest/Stein, S. $670 \mathrm{ff}$.

114 Vgl. Moore, S. 1 ff.; Shannon, Philosophical Magazine, Ser. 7 (1950), Vol. 41, 256, $264 \mathrm{ff}$. 
in den genannten Beispielen also systematisch das Labyrinth oder die möglichen Schachzüge berechnen. ${ }^{115}$ Sobald das Ende des Labyrinths bzw. ein vorteilhafter Zug gefunden wurde, wird dieser ausgeführt. Problematisch ist, dass ein solcher Algorithmus schnell an seine Grenzen gerät, wenn dessen Wachstum schneller als polynomielles Wachstum ${ }^{116}$ ist - wie beispielsweise bei Schach - und damit sowohl die nötige Rechenzeit als auch der notwendige Speicherbedarf unpraktikabel werden. ${ }^{117}$ Nichtsdestotrotz ist die Suchstrategie ein weitverbreitetes Werkzeug, welches - sofern das Problem nicht zu komplex oder kompliziert wird - stets die beste Lösung findet. Allerdings besitzen Suchstrategien kein Gedächtnis, welches ihnen erlauben würde, den gelernten Weg wiederzuverwenden.

\section{Entscheidungsbäume}

Bei einem Entscheidungsbaum handelt es sich um eine Kette von WennDann-Entscheidungen. ${ }^{118}$ Vereinfacht ist dies dem Juristen bei der Fallprüfung bekannt. Nimmt man als (vereinfachtes) Beispiel die Prüfung der zivilrechtlichen Stellvertretung, dann muss eine eigene Willenserklärung, die im Namen eines anderen mit Vertretungsmacht abgegeben wurde, geprüft werden (Abbildung 1). Sobald eines der Elemente nicht bejaht werden kann, führt die Prüfung zu dem Ergebnis, dass eine wirksame Stellvertretung nicht vorliegt. ${ }^{119}$

115 Shannon, Philosophical Magazine, Ser. 7 (1950), Vol. 41, 256, 259.

116 Beispielsweise exponentielles Wachstum.

117 Vgl. Cormen/Leiserson/Rivest/Stein, S. $1059 \mathrm{ff}$.

118 Söbbing, CR 2020, 223, 224 nimmt den Entscheidungsbaum als grundsätzliche Methode an, Algorithmen darzustellen, reduziert ihn allerdings auf seine grafische Darstellung.

119 Das Schema ist hier bewusst vereinfacht worden, um die Grafik nicht zu überfrachten. 


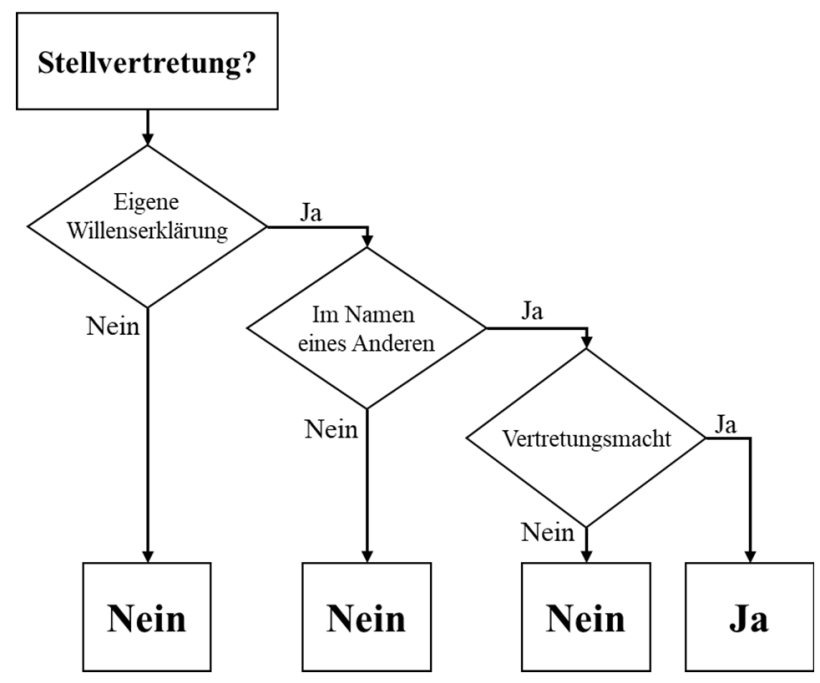

Abbildung 1: Das (vereinfachte) Prüfungsschema als Illustration eines Entscheidungsbaums

Bei den Entscheidungsbäumen wird eine Reihe von numerischen Entscheidungen zur Bestimmung eines Ergebnisses genutzt, was gewiss - genauso wie reale Stellvertretungsprüfung - komplizierter ist. In Abgrenzung zu den Suchstrategien müssen Entscheidungsbäume zum Erreichen des Zieles den Suchraum nicht ständig neu durchlaufen. Ein Entscheidungsbaum speichert die Schwellenwerte und deren Reihenfolgen. Das so ermittelte Regelwerk kann deswegen wiederverwendet werden, weswegen die Methode des Anlernens von Entscheidungsbäumen nicht nur zu der Gruppe der Künstlichen Intelligenz zählt, sondern auch zum Machine Learning. Der Entscheidungsbaum soll nachfolgend an einem der bekanntesten Beispielaufgaben des Machine Learning illustriert werden, dem sog. »Titanic Datenset« der Universität Stanford. ${ }^{120}$

120 Stanford University, Titanic Dataset, »A Titanic Probability«, verfügbar unter: https://web.stanford.edu/class/archive/cs/cs109/cs109.1166/problem12.html (zuletzt abgerufen am 01.06.2020). 


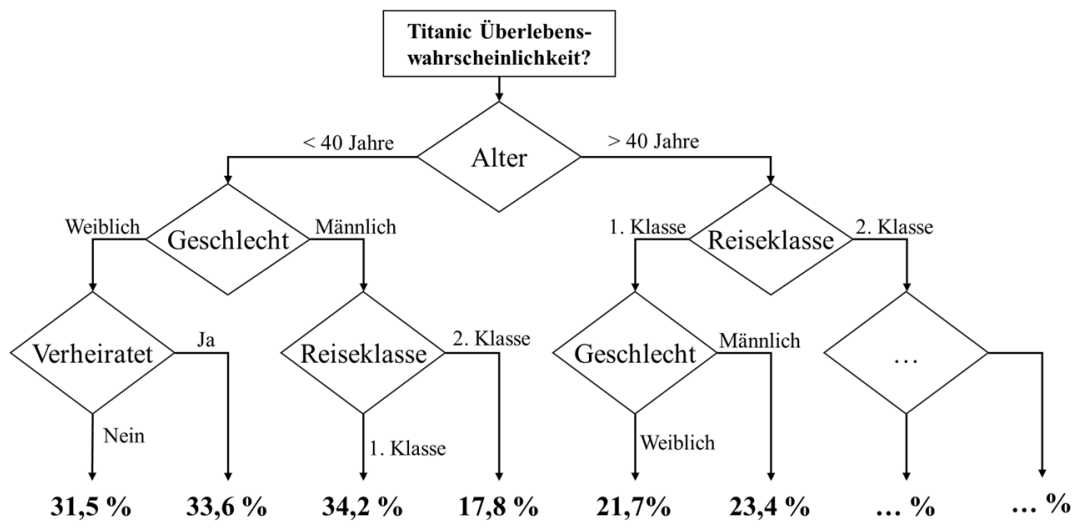

Abbildung 2: Schematisches Beispiel eines Entscheidungsbaums des Titanic Datensets

Der Titanic Datensatz ist im Kern eine Liste von 887 Passagieren, welche sich zum Zeitpunkt der Jungfernfahrt im Jahre 1912 an Bord befanden. Neben dem Namen der Person enthält diese Liste unter anderem auch Informationen über Reiseklasse, das Geschlecht, das Alter sowie die Information, ob die Person das Schiffsunglück überlebt hat. Die Aufgabe ist nun üblicherweise, einen Entscheidungsbaum zu ermitteln, welche die Überlebenswahrscheinlichkeit einer Person prognostizieren kann. Hierzu wird zu jeder Eigenschaft ein Schwellenwert gesucht, bei dessen Anwendung das Datenset möglichst zutreffend aufgeteilt wird. So kann es eine bestimmte Altersgrenze geben, ab der ein Überleben zunehmend (un)wahrscheinlich wird. Gleiches gilt für die Frage des Geschlechts oder der Reiseklasse. Während des Lernprozesses, werden diese Schwellenwerte sowie die Reihenfolge, nach der sie optimalerweise abgefragt werden, sukzessive ermittelt. Mit Abschluss des Lernprozesses werden die Entscheidungsregeln sowie Schwellenwerte abgespeichert und können anschließend zur Ermittlung der Überlebenswahrscheinlichkeit genutzt werden (Abbildung 2).

Obgleich dieses Beispiel sehr realitätsfern wirkt, so illustriert es doch die Funktionsweise von Entscheidungsbäumen. Beispielsweise finden sich bei Versicherungen, Entscheidungsbäume, welche anhand von Versicherungsdaten aus der Vergangenheit erlernt werden und dessen Ergebnisse auf neue Kunden übertragen werden. ${ }^{121}$ Auf diese Weise kann bei Lebensver-

121 Vgl. Frempong/Nicholas/Boateng, International Journal of Statistics and Applications $2017,117 \mathrm{ff}$. 
sicherungen das voraussichtliche Alter der Versicherungsnehmer abgeschätzt werden. Im Bankwesen kann mit Entscheidungsbäumen die Kreditwürdigkeit sowie der angemessene Zinssatz eines Kreditinteressenten ermittelt werden. ${ }^{122}$ Hierbei ist die Eigenschaft, dass die Entscheidungsprozesse für den Menschen nachvollziehbar sind, ein entscheidender Vorteil im Gegensatz zu vielen anderen Methoden der Künstlichen Intelligenz. $^{123}$

\section{Clustering-Algorithmen}

Clustering-Algorithmen sind eine sehr alte Form der Künstlichen Intelligenz und zählen - ebenso wie die Entscheidungsbäume - zu den Methoden des Machine Learning. Ziel dieser Algorithmen ist es, zusammenhängende Gruppen (sog. »Cluster«) in Datenpunkten zu identifizieren (Abbildung 3). ${ }^{124}$

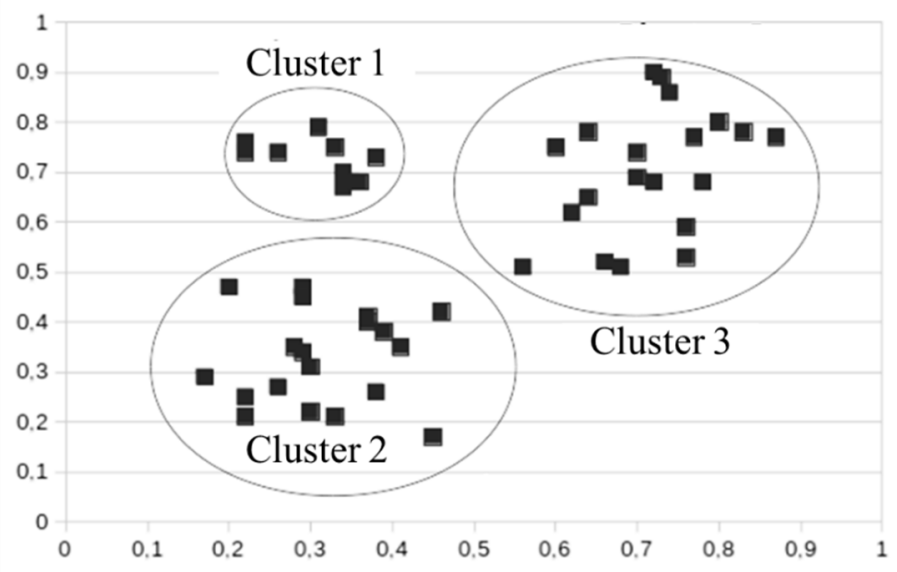

Abbildung 3: Punkte, welche in drei Cluster unterschieden werden können.

Ein geeignetes Beispiel für Clustering-Algorithmen ist die Warenkorbanalyse im Handel. ${ }^{125}$ Hierbei werden die Warenkörbe in Bezug auf deren Zusammensetzung in Verbindung mit weiteren verfügbaren Daten -

122 Vgl. Jiang, in: WRI, S. 18 ff.

123 Vgl. Arrieta/Días-Rodríguez/Del Ser et al., S. 14 ff., 28.

124 Vgl. Omran/Engelbrecht/Salman, Intelligent Data Analysis 11 (2007), 583.

125 Vgl. Liu/Lee/Mu, Knowledge Management Research 19/4 (2018), 59 ff. 
beispielsweise Kundendaten - geclustert. Werden beispielsweise Nutzerdaten eines Online-Shops gespeichert, versucht ein Clustering-Algorithmus nun innerhalb dieser Daten Gruppen zu identifizieren, sodass den Nutzern für sie spezifische Waren angeboten werden können mit dem Ziel, die Verkaufswahrscheinlichkeit der angebotenen Artikel kontinuierlich zu erhöhen.

\section{Fuzzylogiken}

Im Gegensatz zur bekannten klassischen Logik mit ihren beiden (booleschen bzw. binären) Werten »wahr« und »falsch «, ${ }^{126}$ ist die Fuzzylogik ${ }^{127}$ eine unscharfe Logik, die zwischen den sicheren und den garantiert nicht eintretenden Ereignissen jegliche stetige Zwischenwerte annehmen kann. ${ }^{128}$ Im Kern erlauben Fuzzylogiken damit einem Computer, mit unscharfen Informationen umzugehen. Das Anwendungsgebiet von Fuzzylogiken ist schwerpunktmäßig in der Regelungstechnik verortet (sog. FuzzyRegler). ${ }^{129}$ Grundsätzlich sind solche unscharfen Informationen in digitalen Rechnern allerdings problematisch, ${ }^{130}$ da ein Computer für jede Situation einen exakten Zustand einnehmen muss. ${ }^{131}$ Dies verhindert, dass unscharfe Regeln, wie Menschen sie täglich anwenden, sich eins zu eins auf einen Computer übertragen lassen. Nimmt man beispielsweise die Steuerung einer Drohne, die in der Luft stehen und das Gleichgewicht halten soll, sind die Regeln hierzu verbal einfach formuliert: Wenn sich die Drohne zu weit nach links bewegt, soll sie mehr nach rechts fliegen. Wenn sie zu hoch ist, soll sie sinken etc. Für den Flugregler der Drohne - der letzten Endes nur darüber entscheiden kann, ob er einen der Motoren stärker oder schwächer antreibt - ergeben sich dadurch eine Reihe von Regeln, welche es zu erfüllen gilt. Problematisch ist nun, dass der Flugregler erkennen muss, wie hoch zu hoch sein soll. An diesem Punkt setzen Fuzzylogiken an, welche anhand von einfachen Funktionen (den sog. »Zugehörigkeitsfunktionen«) diese Zuordnungen ermöglichen. Die Zugehörigkeitsfunktionen weisen den (unscharfen) Zuständen einen Wert zu, sodass mithilfe dieser Werte entsprechend geregelt werden kann. Ist beispielsweise die Drohne zu $70 \%$ in

126 Vgl. Schnapp, S. 13 ff.

127 Vom englischen Begriff »fuzzy«, zu Deutsch »verwischt«.

128 Dassow, S. 123.

129 Vgl. Thomas, S. 168.

130 Vgl. Ansari, Computer Education 88 (1998), 5.

131 Vgl. Hoffmann, Technische Informatik, S. 33 ff. 
der richtigen Höhe und zu 30\% zu hoch, kann durch diese Aufteilung der Flugregler - bei einer an sich binären Entscheidung - auch auf komplexe Flugsituationen differenziert reagieren, indem er beispielsweise die Motorleistung mit rund einem Drittel seiner möglichen Intensität anpasst. Der Vorteil von Fuzzylogiken ist es, Maschinen auf eine verhältnismäßig einfache und intuitive Weise zu regeln. Schon seit einiger Zeit finden Fuzzylogiken Anwendung in Autopiloten der Luftfahrt ${ }^{132}$ oder in Antiblockiersystemen von Kraftfahrzeugen. ${ }^{133}$ Allerdings wird diese Technologie zunehmend auch in einfachen Haushaltsgeräten benutzt: Fuzzylogiken werden in modernen Waschmaschinen eingesetzt, um beim Waschen den Verschmutzungsgrad der Wäsche abzuschätzen und Waschvorgänge zu optimieren. $^{134}$

\section{Semantic Web Systeme/Expertensysteme}

Die bisher dargelegten Methoden zeichnen sich dadurch aus, dass sie zur Lösung von Problemen herangezogen werden, welche kein oder wenig Wissen erfordern. Das stellt sich bei komplizierten Fragestellungen anders dar, wie sie beispielsweise in einer Quizshow gestellt werden. Für die Arbeit mit vernetzten Informationen eignen sich Semantic Web Systeme bzw. Expertensysteme. ${ }^{135}$ Kern eines solchen Systems ist ein Wissensgraph, ${ }^{136}$ welcher Entitäten und Klassen in einen logischen Zusammenhang bringt und diese durch einen Computer ausgelesen werden können. Ein exemplarischer Ausschnitt eines Wissensgraphen ist in Abbildung 4 skizziert. ${ }^{137}$ Er bildet ein paar wenige beispielhafte Informationen über die beiden Physiker Albert Einstein und Vera Kistiakowsky ab, wie den Geburtsort, den Beruf oder in welchem Staat sich ein für die Personen relevanter Ort befindet.

132 Vgl. Luo/Lan, in: Li/Gupta, S. 90 ff.

133 Unlusoy/Yazicioglu, Int. J. Vehicle Design, Vol 48(3), $1 \mathrm{ff}$.

134 Agarwal, S. 2 ff.

135 Vgl. Davies/Warren, in Domingue/Fensel/Hendler, S. $737 \mathrm{ff}$.

136 Teils wird in der Literatur auch von einer Ontologie gesprochen.

137 Es handelt sich hier um eine vereinfachte Darstellung. Damit ein Computer einen Wissensgraphen durchsuchen kann, muss dieser eine spezielle Syntax verwenden. Hierbei bedient man sich üblicherweise dem Ressource Description Framework (RDF) oder in komplexen Fällen der Web Ontology Language (OWL), s. u., Wissen und dessen Repräsentation (S. 66). 


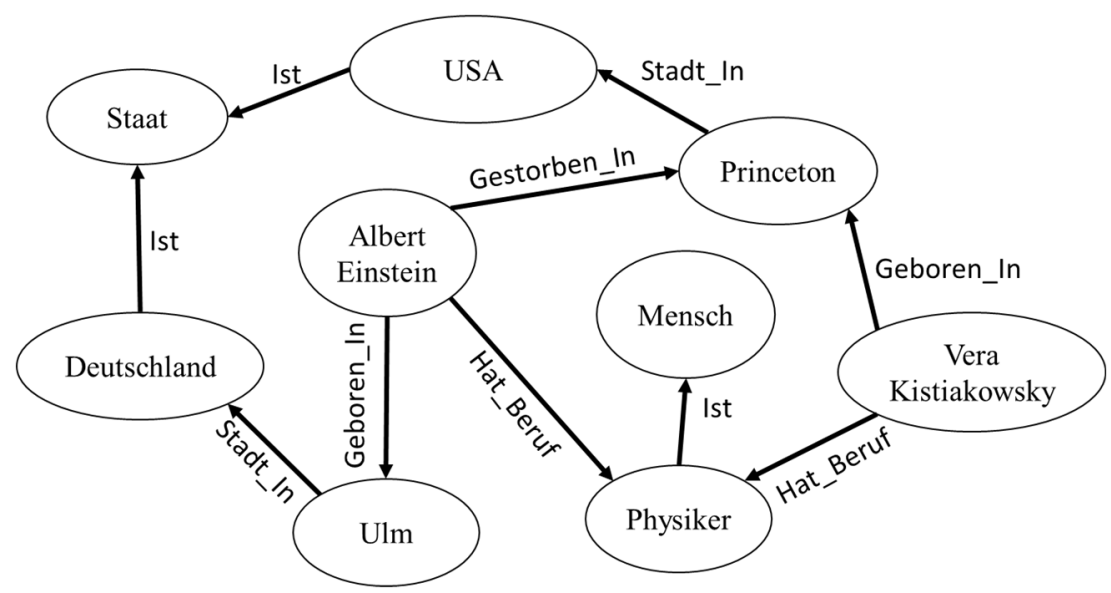

Abbildung 4: Beispiel eines einfachen Wissensgraph

Obgleich dieser Wissensgraph sehr trivial gehalten ist, sind bereits einfache maschinelle Abfragen möglich. Es kann nach dem Geburtsort von Albert Einstein oder nach dem Beruf von Vera Kistiakowsky gefragt werden. Das KI-System würde in diesem Fall »Ulm» und »Physiker« ausgeben. Gleichzeitig ist der Wissensgraph ausreichend komplex, dass erste Schlussfolgerungen ${ }^{138}$ durchgeführt werden können. Möglich sind Abfragen nach der Stadt, in der ein Physiker gestorben und ein weiterer Physiker geboren wurde. Der sog. »Reasoning-Algorithmus« wird den Wissensgraphen nach einer Entität durchsuchen, die der Frage genügt und dabei auch Aussagen des Wissensgraphen kombinieren. ${ }^{139} \mathrm{Im}$ vorliegenden Beispiel wäre die Ausgabe »Princeton«

Es ist zu beachten, dass für einen außenstehenden Dritten - der keinen Einblick in den Wissensgraphen hat - ein solches KI-System den Eindruck erweckt, dass es die Aussagen und Konzepte versteht. Es wirkt daher so, als hätte die Künstliche Intelligenz ein Verständnis von dem Konzept eines Physikers, einer Person oder eines Ortes. Das Verhalten des KI-Systems hängt aber nur von dem zugrunde liegenden Wissensgraphen ab. Wird im oben gezeigten Wissensgraphen beispielsweise danach gefragt, was »Deutschland « sei, wird lediglich das ausgegeben, auf was der Zeiger »Ist « zeigt, also »Staat«. Ein Verständnis dieser Begriffe liegt aber nicht vor.

138 Auch »Reasoning« genannt.

139 Diese Algorithmen verwenden meist eine Form der Suchstrategien, s. o., Suchstrategien (S. 34). 
Insofern ähnelt diese Form der Künstlichen Intelligenz einer sehr komplexen Datenbankabfrage. Dennoch lassen sich - sofern die Wissensgraphen sehr umfangreich sind - sehr komplexe Schlussfolgerungen ziehen, welche auch jenseits der menschlichen Fähigkeiten liegen können. So kennt der Wissensgraph »YAGO 4« über 10.000 Klassen und über 300 Millionen Fakten. ${ }^{140}$ Die einfache Maschinenzugänglichkeit der Information machen sich auch Suchmaschinen wie »Google« und »Yahoo!« mit eigenen Wissensgraphen zunutze, um Informationen bereit $\mathrm{zu}$ stellen. ${ }^{141} \mathrm{KI}-$ Systeme wie diese bilden das Fundament für komplexe Programme wie das IBM Watson System, welches in der Lage ist, sich bei einer Quizshow gegen Menschen zu behaupten. ${ }^{142}$ Es ist jedoch zu beachten, dass gegenwärtig Wissensgraphen mit einer hohen Qualität nicht ohne Zutun des Menschen erstellt werden können, ${ }^{143}$ was einer weiten Verbreitung dieser Technologie bisher entgegen steht.

\section{Evolutionäre Algorithmen}

Eine exotischere Form der Künstlichen Intelligenz sind die evolutionären Algorithmen. Wie der Name vermuten lässt, sind evolutionäre Algorithmen von biologischen Prozessen in der Natur inspiriert und versuchen deren Strategien zum Bewältigen von Optimierungsproblemen nachzuahmen. Ein evolutionärer Algorithmus läuft in drei Schritten ab: Initiierung, Evaluation und Optimierung. Das lässt sich am (vereinfachten) Beispiel des Berechnens einer optimalen Antennengeometrie aus einem einfachen Stück Draht veranschaulichen. ${ }^{144}$ Das Ergebnis eines solchen Prozesses ist die in Abbildung $5^{145}$ gezeigte Antenne, ${ }^{146}$ die im Rahmen einer NASA-Mission entwickelt wurde und sich unter anderem durch eine bessere Sende- und Empfangsleistung bei einem gleichzeitig sehr simplen Design und einer verkürzten Entwicklungszeit auszeichnet. ${ }^{147}$ Daran zeigt sich, dass evolutionäre Algorithmen bei solchen Ansätzen problemlos Lösungen finden

140 Pellissier-Tanon/Weikum/Suchanek, The Semantic Web 2020, 583, 594.

141 Paulheim, The Semantic Web 2017, 589, 493.

142 Gliozzo/Ackerson/Bhattacharya et al., S. 30.

143 Pellissier-Tanon/Weikum/Suchanek, The Semantic Web 2020, 583, 586

144 Vgl. Hornby/Lohn/Linden, Evolutionary Computation 19 (2011), 1 ff.; Dieses Beispiel findet sich schon bei Konertz/Schönhof, ZGE/IPJ 10 (2018), 379, 398 ff.

145 Entnommen aus Lohn/Hornby/Linden, AI EDAM 22 (2008) 235, 242.

146 Vgl. U.S. Patent Nr. 5,719,794.

147 Hornby/Lohn/Linden, Evolutionary Computation 19 (2011), 1, 20. 
können, die unter objektiver Betrachtung als (patentrechtliche) Erfindungen gelten würden. ${ }^{148}$

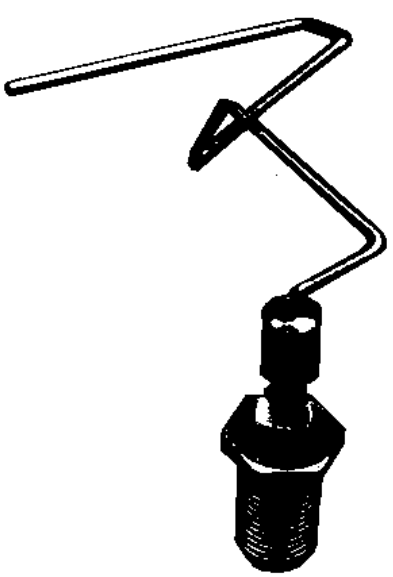

Abbildung 5: Eine evolutionär designte Antenne für einen Satelliten

Damit ein evolutionärer Algorithmus eine Lösung optimieren kann, ist die Grundvoraussetzung, dass ein - üblicherweise ungenügender - Lösungsansatz existiert; im Beispiel der Antenne ein Stück Draht. Dieser Lösungsansatz wird bei der Initiierung zufällig modifiziert; beim Antennenproblem werden also zufällige Antennengeometrien generiert. Diese Antennengeometrien stellen ein Stück Draht mit einem zufälligen Knick dar. Die so genierten Lösungen werden anschließend evaluiert, ${ }^{149}$ wobei sie sich nach wie vor als ungenügend erweisen werden. Dennoch unterscheiden sich die Ergebnisse voneinander und manche Antennengeometrien werden weniger schlecht bzw. minimal besser sein als andere.

An diesem Punkt beginnt nun die Optimierung durch den evolutionären Algorithmus. Dabei können vier Phasen unterschieden werden: Selektion, Rekombination, Mutation und Evaluation, die - in Schleifen - immer wieder durchlaufen werden. Zunächst werden die schlechtesten Lösungen entfernt (Selektion). An deren Stelle tritt eine Kreuzung aus den Lösungsvarianten, welche verblieben sind (Rekombination). Dadurch wird die Menge der Lösungen, die durch die Selektion verkleinert wurde, wieder aufgefüllt, sodass die Anzahl der Lösungen mit der Anzahl nach der

149 Üblicherweise durch eine Simulation. 
Initiierung identisch ist. Damit sich die Lösungen nun über eine simple Kreuzung hinaus weiterentwickeln können, bedarf es im Weiteren einer Änderung der Lösungen. Dafür wird ein Teil der Lösungen (pseudo-)zufällig und geringfügig verändert (Mutation). Dieser neue Satz an Lösungen wird nun erneut evaluiert und der Prozess beginnt so lange von vorne, bis die Evaluation die gewünschte Qualität erreicht oder weitere Durchläufe zu keiner signifikanten Verbesserung führen. ${ }^{150} \mathrm{Im}$ Antennenbeispiel bedeutet dies, dass die zufällig generierten und anschließend evaluierten Antennen zunächst selektiert werden. Die fehlenden Antennengeometrien können nun entweder durch Kombination erzeugt oder durch Kopieren der besten Antennengeometrien ersetzt werden. Im Anschluss wird ein Teil der Antennengeometrien geringfügig mutiert, beispielsweise durch geringfügige Änderungen des Biegewinkels an einer beliebigen Stelle. Diese neuen Antennengeometrien werden abschließend in einer Simulation evaluiert und bewertet. Mit der anschließenden Selektion beginnt der Durchlauf erneut. Mit jedem Durchlauf führen kleine zufällige Änderungen dazu, dass sich die Leistung der Antennen verändert. Durch den fingierten »evolutionären Druck« wird sich stets die leistungsfähigste Antenne behaupten.

\section{Zwischenergebnis}

Es lässt sich festhalten, dass es eine Vielzahl von KI-Methoden gibt, die an dieser Stelle nicht abschließend dargelegt wurden. Es kann auch bezweifelt werden, dass aufgrund des schnellen Fortschritts in der Entwicklung der KIMethoden derzeit überhaupt eine abschließende Darstellung erfolgen kann. Gezeigt wurde auch, dass die verschiedenen Methoden, unterschiedliche Ansätze zur Lösung unterschiedlicher Probleme aufweisen. Hinzu kommt, dass es möglich ist, ein Problem mit unterschiedlichen KI-Methoden zu lösen. Daher lassen sich an dieser Stelle zwei wichtige Aspekte festhalten: Erstens, es gibt nicht »die« Künstliche Intelligenz und zweitens gehören nur manche KI-Methoden zum sog. "Machine Learning«. Deshalb ist Künstliche Intelligenz eben nicht in jedem Fall selbstlernend. Streng von den KI-Methoden zu trennen sind »Big Data«-Methoden, welche primär darauf abzielen, große und heterogene Datenmengen zu verwalten. ${ }^{151} \mathrm{KI}-$ Methoden sind zwar nicht selten datengetrieben und müssen daher eine

150 Vgl. Wong, S. 2.

151 Engels/Goecke, S. $6 \mathrm{ff}$. 
Aufgabe, wie bei Entscheidungsbäumen oder Clusteralgorithmen, erst lernen, sodass große Datenmengen aus Big Data Systemen hierbei hilfreich sind. Es handelt sich aber bei Künstlicher Intelligenz und Big-Data um verschiedene Konzepte.

III. Beispiel: (Künstliches) neuronales Netz

Aufgrund der kontinuierlich anwachsenden Bedeutung des autonomen Fahrens, der Sprachverarbeitung, der Bilderkennung oder des automatischen Handels mit Wertpapieren soll nachfolgend jene KI-Methode ausführlicher dargestellt werden, die diesen Trend maßgebend ermöglicht hat: Die (künstlichen) neuronalen Netze. ${ }^{152}$

\section{Modelldarstellung}

Vereinfacht kann ein neuronales Netz näherungsweise mit einem Thermostat respektive Drehregler an der heimischen Heizung verglichen werden (Abbildung 6). ${ }^{153} \mathrm{Je}$ weiter der Regler in eine Richtung gedreht wird, desto wärmer wird ein Raum und je weiter er in die andere Richtung gedreht wird, desto kälter wird der Raum. Jede nennenswerte Abweichung der Zimmertemperatur vom Komfortwert wird der Bediener in Form von Schwitzen bzw. Frösteln erfahren. Bereits ein Kind ist in der Lage, den Zusammenhang zwischen der Stellung des Thermostats und der Zimmertemperatur zu ermitteln. Nach einer kurzen Zeit des Probierens ist die passende Stellung ermittelt worden und wird fortan beibehalten (sog. »Training«). 


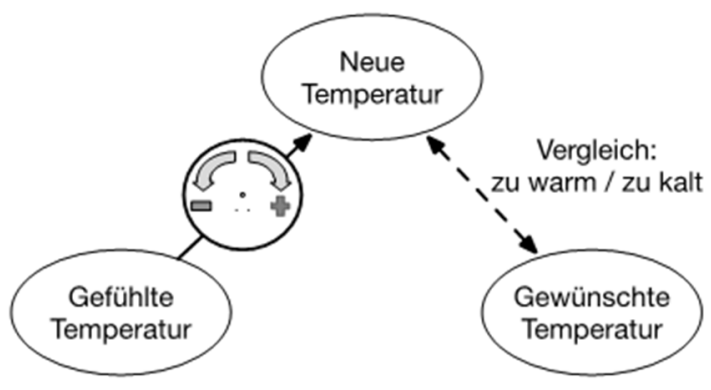

Abbildung 6: Das Einstellen eines Thermostats als Analogie für den Lernprozess (künstlicher) neuronaler Netze

Auf genau diese Weise lernt auch ein neuronales Netz. Statt der gefühlten Temperatur, welche in Form einer einzigen Zahl beschrieben wird, kann ein neuronales Netz viele Werte aufnehmen. ${ }^{154}$ Gleichsam kann statt eines einzigen Ergebnisses (die neue Temperatur) auch eine Vielzahl von Werten resultieren. ${ }^{155}$ Zudem wird eine enorme Menge an virtuellen Thermostaten verwendet (in der Informatik werden diese als Gewichte bezeichnet), welche den Input mit dem Output über Multiplikationen und Additionen zu einem Netz verflechten. In Abbildung 7 ist ein einfaches neuronales Netz abgebildet, welches aus neun Neuronen bzw. Knoten (N1-N9), davon vier Inputneuronen, zwei Zwischenneuronen und drei Outputneuronen besteht, die über insgesamt 14 gewichtete Kanten (Linien) verbunden sind.

154 Die Gesamtheit aller Zahlenwerte, die in das Netz geleitet werden, werden Inputdaten genannt.

155 Die Gesamtheit aller Zahlenwerte, die aus dem Netz geleitet werden, werden Outputdaten genannt. 


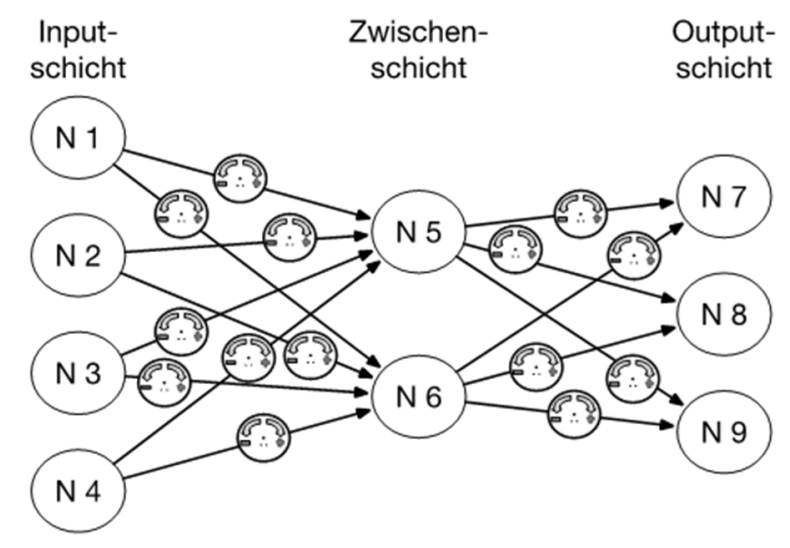

Abbildung 7: Schematischer Aufbau eines einfachen neuronalen Netzes

Sobald die Inputwerte bekannt sind, kann über die Gewichte der Kanten zunächst der Wert der Zwischenneuronen und anschließend der Wert der Outputneuronen berechnet werden. Welche (reale) Information letztlich durch die Neuronen repräsentiert wird, ist nicht relevant. Es kann sich um ein Sensorsignal, eine Tonfolge oder ein Bild ${ }^{156}$ handeln. Gleichwohl kann der Output unterschiedliche Bedeutung haben, beispielsweise ein Bild, eine Kategorie ${ }^{157}$ oder - wie beim autonomen Fahren - Signale an die Lenkung, den Motor und die Bremse. Mit steigender Anzahl an Neuronen und Schichten ist das Netz in der Lage, immer kompliziertere Zusammenhänge zwischen dem Input und dem Output abzubilden.

\section{Trainingsphase}

Wird ein neuronales Netz erstellt, sind die Gewichte der Knoten zu Beginn nicht bekannt und werden daher mit zufälligen Werten ${ }^{158}$ belegt; es handelt sich um ein untrainiertes, neuronales Netz. Bezugnehmend auf die Analogie

156 Ein Netz, welches HD-Bilder in Farbe verarbeiten kann, benötigt beispielsweise drei Neuronen für jedes Pixel und somit schon $1920 * 1080 * 3=6.220 .800$ Inputneuronen.

157 Beim Klassifizieren wird üblicherweise jedem Neuron eine Klasse zugeordnet. In einem neuronalen Netz zur Bilderkennung ist beispielsweise je ein Neuron einer Tierart zugeordnet.

$158 \mathrm{Zu}$ der Problematik von Zufall in Computersystemen, s. u., Randomisierte Algorithmen und Pseudozufall (S. 54). 
des Heizungsthermostats sind zu Beginn sämtliche Reglerstellungen komplett zufällig gewählt worden. Daraus folgt, dass mit einem untrainierten Netz noch keine Aufgaben erfüllt werden können. Damit ein neuronales Netz eine Aufgabe ausführen kann, muss es diese zunächst erlernen (sog. »Trainingsphase «). Ziel ist es, eine geeignete Stellung für jeden Regler in dem neuronalen Netz zu finden, der bei einem gegebenen Inputwert zu dem gewünschten Outputwert führt. Dies erfolgt im einfachsten Fall mit gelabelten Trainingsdaten. ${ }^{159}$ Solche Trainingsdaten bestehen aus einem vordefinierten Input und einem Output. Bei sehr einfachen selbstfahrenden Autos kann der Input beispielsweise aus den Kamerabildern, der Geschwindigkeit, der Beschleunigung und den GPS-Koordinaten bestehen. Als Output dient der Lenkradeinschlag, das Brems- und das Motorsignal. Der Optimierungsalgorithmus versucht nun die Gewichte in dem neuronalen Netz so anzupassen, dass der aus dem Input berechnete Output möglichst dem tatsächlich gemessenen Output entspricht. Übertragen auf das Thermostatbeispiel bedeutet dies, dass der Optimierungsalgorithmus anhand von bekannten Sollausgabewerten (im Beispiel die Zieltemperaturen) neue Gewichte berechnet und entsprechend verändert, damit das Resultat ein bisschen besser ausfällt. Falls sich bei nachfolgenden Daten herausstellt, dass sich der Unterschied verringert hat, wird der Optimierungsalgorithmus versuchen, die Gewichte weiter zu verstärken. Dieser Prozess wiederholt sich so lange, bis der Optimierungsalgorithmus keine weitere Verbesserung erreichen kann. Die so gefundenen Gewichtswerte werden nach dem Training nicht mehr verändert und anschließend abgespeichert. ${ }^{160}$

Nachdem ein Netz trainiert wurde, ist es in der Lage, einem verhältnismäßig hohen Anteil an Inputwerten aus den Trainingsdaten die richtigen Outputwerte zuzuordnen. Die Leistungsfähigkeit wird anschließend im Rahmen der Evaluation ermittelt. Hierbei wird geprüft, ob auch Inputwerte, mit denen das Netz nicht trainieren konnte, zu korrekten Outputwerten führen. Dieser Schritt ist notwendig, da immer die Gefahr besteht, dass das Netz nicht die Zusammenhänge, sondern lediglich das Trainingsset »auswendig« lernt (sog. »Overfitting «). ${ }^{161}$ In diesem Fall könnte es zwar die Trainingsdaten korrekt klassifizieren, es würde allerdings an neuen, unbekannten Inputs scheitern. Um dies zu verhindern, wird üblicherweise nicht mit dem gesamten Trainingsset trainiert, sondern es

159 Alternativ kann in manchen Anwendungsfällen auch aus ungelabelten Daten gelernt werden. Dies wird grundsätzlich angestrebt, da das Labeln nur manuell durchgeführt werden kann und verhältnismäßig zeitintensiv ist.

160 Vgl. Ertel, S. $291 \mathrm{ff}$.

161 Hagan/Demuth/Beale/De Jesús, Kap. 13, S. 3. 
wird eine kleine Menge von Daten zurückbehalten, um anschließend damit das KI-System zu evaluieren. Erst wenn das neuronale Netz auch mit diesen Daten einen ausreichend hohen Anteil korrekt berechnet, kann es genutzt werden. ${ }^{162}$

\section{Anwendungsphase}

Bei der Nutzung des neuronalen Netzes werden die jeweiligen durch das KI-System erfassten Inputdaten an das Netz geleitet, welches die Outputwerte berechnet. Diese werden anschließend genutzt, um entsprechende Steuersignale $\mathrm{zu}$ generieren (sog. "Anwendungsphase« oder »Inferencing «). Im Gegensatz zur Trainingsphase finden hier keine zufälligen Werte mehr Verwendung, sodass Zufall keinen Einfluss auf das Verhalten des KI-Systems hat. ${ }^{163}$ Die für den Outputwert relevanten Gewichte werden nicht mehr geändert. Daraus folgt, dass ein identischer Inputwert stets zu denselben Outputwerten führt.

\section{Sonderfall: Weiterlernende Systeme}

Einen gewissen Sonderfall stellen weiterlernende Systeme dar. Vorab ist anzumerken, dass die soeben genannten Phasen, bestehend aus Trainingsphase und Anwendungsphase auf einem neuronalen Netz nicht zeitgleich ablaufen können. Entweder werden die gefundenen Gewichte zur Berechnung eines gegebenen Inputs (Anwendungsphase) genutzt oder es werden bessere Gewichte gesucht (Trainingsphase). Das heißt aber nicht, dass weiterlernende Systeme nicht möglich sind. Bei einem nicht weiterlernenden, starren System (sog. »Batch Learning «) läuft die Trainingsphase nur einmal ab. Die mit dem dabei verwendeten Datensatz (sog. »Batch«) ermittelten Gewichte werden anschließend gespeichert und im Rahmen der Anwendungsphase genutzt. Eine spätere Änderung der Gewichte nach Beginn der Anwendungsphase findet nicht mehr statt. ${ }^{164}$ Der Vorteil eines solchen Vorgehens liegt in einem hohen $\mathrm{Ma}$ an Kontrolle für den

162 Vgl. Hagan/Demuth/Beale/De Jesús, Kap. 22, S. 27 ff.

163 Als Ausnahme von diesem Grundsatz sind hier allenfalls die sog. »Generative Adversarial Networks« (GAN) zu nennen, wobei auch bei diesen nicht die Gewichte selbst verändert werden, sondern aus einem pseudozufälligen Input-Seed ein Output generiert wird.

164 Kriesel, S. 54. 
Hersteller oder Entwickler über sein neuronales Netz. Ein einmalig angelerntes neuronales Netz wird sein Verhalten in der gleichen Situation also bei der gleichen Eingabe - während der späteren Anwendung nicht ändern.

Bei einem weiterlernenden System (sog. »Online Learning «) lernt das KI-System während der späteren Anwendung weiter, womit sich die Kantengewichte auch nach der ersten Trainingsphase ändern (können). Dennoch findet dies nicht während der Anwendungsphase statt, vielmehr sind auch hier - wie zu Beginn genannt - die Lern- und Anwendungsphasen zu trennen. Entweder wechseln sie sich (in einer Endlosschleife) ab oder sie werden parallel, aber mit zunächst getrennten künstlich neuronalen Netzen (in sog. »Threads«) ausgeführt. ${ }^{165}$ Die Inputs (Dateneingaben) des neuronalen Netzes in der Anwendungsphase werden protokolliert und für zukünftige Trainingsphasen verwendet. Sobald die Trainingsphase zu einer besseren Gewichtskombination geführt hat, werden diese neuen berechneten Gewichte des neuronalen Netzes nach der (vorherigen) Trainingsphase zwischengespeichert und regelmäßig in das neuronale Netz der Anwendungsphase übernommen. Damit ist das KI-System in der Lage, sich an neue Situationen anzupassen und sich kontinuierlich $\mathrm{zu}$ verbessern. ${ }^{166}$ Problematisch ist jedoch, dass der Hersteller bzw. Entwickler weniger Kontrolle über das neuronale Netz hat, da sich das Verhalten auch ändern kann, wenn das neuronale Netz den Einflussbereich des Herstellers oder Entwicklers verlassen hat. Denn während ein Batch Learning System nach der Trainingsphase bis zu einem gewissen Grad getestet und verifiziert werden kann, ist dies beim Online Learning schwer bis unmöglich. ${ }^{167}$

Als anschauliches Beispiel dient der von Microsoft entwickelte Chatbot Tay. Dieser sollte von der Interaktion mit seinen Nutzern lernen und so seine Fähigkeit, zu kommunizieren, kontinuierlich verbessern. Ein solches lernendes System kann aber das Erlernte nicht selbstständig evaluieren und ist daher auf das Feedback seiner Umgebung angewiesen. Im Falle von Tay bestand dieses Umfeld jedoch aus seinen Nutzern, welche dem System allerdings rassistische und antisemitische Aussagen vorgaben. Daher wandelte sich Tay von einem einfachen Chatbot in einen nationalsozialistischen Parolen-Generator. ${ }^{168}$

165 Vgl. Trobec/Slivnik/Bulic/Robic, S. 50; Ben-Nun/Hoefler, S. 1:8 ff., 1:22 ff.

166 Sahoo/Pham/Lu/Hoi, S. 1 ff.

167 Sahoo/Pham/Lu/Hoi, S. 1.

168 Wolf/Miller/Grodzinsky, ACM SIGCAS Computers \& Society, Volume 47 No. 3 (2017), $54 \mathrm{ff}$. 
Während das Problem des Online Learnings im Falle eines Chatbots noch als einfaches Marketingdebakel verbucht werden kann, stellt es bei sicherheitskritischen Systemen ein fundamentales Dilemma dar. Soll beispielsweise ein autonom fahrendes Fahrzeug kontinuierlich lernen, sicherer zu navigieren, indem es aus vergangenen Fahrsituationen lernt, wäre ein systematischer Fehler, wie bei Tay, nicht akzeptabel. Eine Lösung zwischen Batch Learning und Online Learning stellen wiederkehrende Updates dar. Hierbei werden während der Laufzeit Daten erfasst und das Trainingsset kontinuierlich erweitert, was wiederum die Qualität des Netzes begünstigt. Nach einer umfassenden Evaluation durch den Entwickler bzw. Hersteller des neuen neuronalen Netzes bzw. einer Freigabe eines Qualitätssicherungsprozesses kann dies - als Update - an die Endgeräte bereitgestellt werden (sog. »Rollout «). ${ }^{169}$

\section{Black-Box Konzept}

Neuronale Netze werden oft als »Black-Box« bezeichnet. ${ }^{170}$ Wie bereits deutlich wurde, sind die Rechenschritte, mit denen ein neuronales Netz einen Output aus einem Input berechnet, nicht unbekannt. Ein neuronales Netz ist somit keine »Black-Box« bezogen auf die informationsverarbeitenden Prozesse, welche im neuronalen Netz ablaufen. ${ }^{171}$ Der BlackBox Gedanke bezieht sich nur auf die semantische Beziehung zwischen den Informationen, die in das Netz eingebracht werden und der Antwort, die man erhält. Analog zu einem neuronalen Netz verhält es sich auch mit einem Kind, das einen Heizkörper zu bedienen gelernt hat. So hat es zwar die Beziehung verinnerlicht und kann diesen Zusammenhang im Alltag nutzen, um für jeden Raum die gewünschte Temperatur zu finden. Es bedarf aber keiner Kenntnis über die Funktionsweise einer Zentralheizung, über Thermodynamik, Mechanik oder Strömungslehre. Der tatsächliche Zusammenhang bleibt dem Kind nach wie vor als »Black-Box« verborgen. Das Problem der Black-Box betrifft allerdings nicht nur neuronale Netze, sondern kann prinzipiell auch jede andere KI-Methode, eigentlich gar jede

169 Freilich kann dies mit datenschutzrechtlichen Problemen verbunden sein, wenn während der Laufzeit beim Anwender erhobene Daten an den Hersteller zwecks Verbesserung übertragen werden, vgl. Problematik bei Kraftfahrzeugen, Lüdemann, ZD 2015, 247 ff.

170 Vgl. Hagan/Demuth/Beale/De Jesús, Kap. 22, 3.; Hoeren/Niehoff, RW 2018, 47, 50 .

171 Ebenso Pasluosta/Chiu, in: Cartwright, S. 183 ff. 
Computersoftware, betreffen. Ein Entscheidungsbaum kann, wie der im vorherigen Abschnitt vorgestellte, ${ }^{172}$ grundsätzlich durch den Menschen nachverfolgt werden. Wird dieser Entscheidungsbaum allerdings zu komplex - mit Tausenden von Parametern - so beginnt die (menschliche) Nachvollziehbarkeit der Ergebnisse ebenfalls schnell zu schwinden. ${ }^{173}$

\section{Multi-Agenten Systeme}

Obgleich jede der zuvor gezeigten KI-Methoden in bestimmten Problembereichen ein hohes Leistungsniveau erreichen kann, so ist jede dieser KIMethoden nach wie vor auf bestimmte Problemtypen spezialisiert. Beispielsweise lässt sich mit einem neuronalen Netz ein Programm zur Objekterkennung gestalten, für das Beantworten von Quizfragen ist diese KI-Methode jedoch ungeeignet. Umgekehrt kann eine Semantic Web Applikation komplexe logische Fragestellungen beantworten, um diese in menschlicher Sprache formulierten Fragen jedoch für die Maschine zu übersetzen, bedarf es wiederum einer anderen Künstlichen Intelligenz wie ein neuronales Netz. Das zeigt, dass zur Lösung einer komplexen Aufgabe - wie das autonome Steuern eines Kraftfahrzeugs ${ }^{174}$ oder wie Unterstützungstätigkeiten im Gesundheitswesen ${ }^{175}$ - zahlreiche Einzelprobleme angegangen werden müssen, für welche wiederum unterschiedliche KIMethoden geeignet sind. Hierbei ist jede implementierte KI-Methode als ein eigenständiges Element und damit als eigenständiger Agent zu betrachten. In der Gesamtheit bilden diese Agenten sog. »Multi-Agenten Systeme«, die Vorteile unterschiedlicher Methoden kombinieren. ${ }^{176}$

\section{Vom Modell über den Algorithmus zur Computersoftware}

Genauso wenig wie sich die Skizze eines Eisenbahnfahrzeugs als Fortbewegungsmittel eignet, ist das Modell eines neuronalen Netzes konkrete Computersoftware. Allen KI-Methoden ist gemeinsam, dass sie zunächst nur ein Modell beschreiben, mit welchem sich Informationen in einer bestimmten Weise abstrakt verarbeiten lassen. Aus diesem Modell wird ein

172 S. o., Entscheidungsbäume (S. 35).

173 Vgl. Arrieta/Días-Rodríguez/Del Ser et al., S. 15.

174 Dafflon/Vilca/Gechter/Adouane, Procedia Computer Science, 51 (2015), 423 ff.

175 Vgl. Isern/Moreno, J Med Syst (2016), 40:43, 6.

176 Vgl. Alkhateeb/Al Maghayreh/Aljawarneh, in: ICISMS, S. 75. 
Algorithmus abgeleitet, der schließlich in einer gewählten, mehr oder weniger hohen Programmiersprache umgesetzt wird, um - nach evtl. Kompilierung - ein auf einem Computer lauffähiges Programm zu erhalten. ${ }^{177}$ Insofern ist der Begriff des Algorithmus sowie dessen Beschränkungen für die Untersuchung des Wesens der Künstlichen Intelligenz von besonderer Bedeutung. ${ }^{178}$ Es muss geklärt werden, was ein Algorithmus überhaupt ist und was ihn von Computersoftware unterscheidet. Zudem spielt in vielen Bereichen der Künstlichen Intelligenz der Zufall eine Rolle, sodass eine besondere Art von Algorithmen, die so genannten »randomisierten Algorithmen«, genauer betrachtet werden müssen.

\section{Allgemeiner Algorithmus-Begriff}

Der Begriff »Algorithmus« wird - wie auch der Begriff »Algebra« - ursprünglich vom Namen des Werks »dixit algrismi«, zu Deutsch etwa »So sprach Khowarizmi«, abgeleitet. ${ }^{179} »$ Khowarizmi « ist der Name des ursprünglichen arabischen Autors, womit »Algorithmus« ein Eigenname ohne eigenständige Bedeutung ist. Er wird seit jeher zur Beschreibung von Vorschriften verwendet, die sich nach vorgegebenen Schritten abarbeiten lassen. Der Begriff Algorithmus wird in diesem Sinne nicht nur für Rechenvorschriften für Computer angewendet, ${ }^{180}$ sondern auch für Vorschriften zur Befolgung allgemeiner vorgegebener Regeln, beispielsweise in der Notfallmedizin; ${ }^{181}$ auch ein Kochrezept kann als Algorithmus (im weiteren Sinne) verstanden werden. ${ }^{182}$ Problematisch ist, dass der Begriff keine einheitliche Definition, auch nicht in der Informatik, gefunden hat. ${ }^{183}$ Ein Standardwerk der Informatik beschreibt den Algorithmus »grob gesprochen [als] wohldefinierte Rechenvorschrift, die eine [...] Eingabe verwendet und eine $[\ldots]$ Ausgabe erzeugt «. ${ }^{184}$ Das heißt, für Algorithmen im Bereich von Computern sind drei wesentliche Elemente notwendig: Die Eingabe, die Ausgabe und eine wohldefinierte Rechenvorschrift. ${ }^{185}$ Die Eingabe ist eine

177 Vgl. Aho/Lam/Sethi/Ullman, S. 3 ff.

178 Hoeren/Niehoff, RW 2018, 47, 49.

179 Klaeren/Sperber, S. 246.

180 Vgl. Klaeren/Sperber, S. 246 ff.; Broy, S. 31; so wohl auch Söbbing, CR 2020, 223, 224.

181 Vgl. Peters/Runggaldier, S. $1 \mathrm{ff}$.

182 Goos, S. $22 \mathrm{ff}$.

183 Güting, S. 1.

184 Cormen/Leiserson/Rivest/Stein, S. 5.

185 Im Ergebnis auch bei Marly, Rn. 29. 
Menge von Daten, mit welcher die Rechenvorschrift vorher definierte Schritte abarbeitet und eine Ausgabe produziert, die selbst wieder eine Menge von Daten ist. ${ }^{186}$ Weitere Voraussetzungen an den Algorithmus, die teilweise diskutiert werden, bestehen - wie im Folgenden noch dargelegt wird - nicht. ${ }^{187}$

Bei den oben als Beispiel genannten neuronalen Netzen ist zwischen dem Anlern- bzw. Trainingsalgorithmus und der konkreten Anwendung des künstlichen neuronalen Netzes selbst zu unterscheiden. Beim Anlern- bzw. Trainingsalgorithmus stellen die zufällig gewählten Kantengewichte die Eingaben dar und die späteren (endgültigen) Kantengewichte sind dessen Ausgabe; der Algorithmus wird somit für die Trainingsphase verwendet. Während der Nutzung des trainierten neuronalen Netzes in der Anwendungsphase bestimmt sich die Ausgabe nach den während des Trainings ermittelten Kantengewichten. Diese sind folglich nach dem Training nur noch Konstanten des späteren Programms und damit unveränderlicher Bestandteil. Der Sonderfall des Online Learnings ändert diesen Prozess nicht, denn beim Lernen während des Einsatzes werden lediglich der Trainingsalgorithmus und der Anwendungsalgorithmus abwechselnd ausgeführt. ${ }^{188}$

\section{Randomisierte Algorithmen und Pseudozufall}

Eine besondere Art der Algorithmen sind sog. " randomisierte Algorithmen «. ${ }^{189}$ Hierbei werden in der Rechenvorschrift Zufallszahlen verwendet, um eine zufällige Entscheidung zu treffen (z.B. in der Trainingsphase von künstlichen neuronalen Netzen). Der Vorteil gegenüber nicht randomisierten Algorithmen ist, dass für bestimmte Probleme eine mögliche Lösung schneller (und damit effizienter) gefunden werden kann. Dies ist aber mit dem Nachteil verbunden, dass das Ergebnis evtl. nur näherungsweise korrekt ist (sog. »Monte-Carlo-Algorithmen«) oder nicht bekannt ist, ob der Algorithmus terminiert ${ }^{190}$ (sog. »Las-Vegas-Algorithmen $«$ ). ${ }^{191}$ Die

186 Cormen/Leiserson/Rivest/Stein, S. 5 ff.

187 Güting, S. 28; so aber Söbbing/Söbbing, S. 8.

188 S. o., Sonderfall: Weiterlernende Systeme (S. 49).

189 Vgl. Motwani, ACM Computer Surveys 8 (1996), 33 ff.

190 S. u. zum Begriff »Terminierung«, Determinismus, Determiniertheit und Terminierung in der Informatik (S. 59).

191 Vgl. Karp, Discrete Applied Mathematics 34 (1991), 165 ff. 
theoretische Informatik nimmt bei der Betrachtung randomisierter Algorithmen den Zufall als gegeben an. Allerdings kann ein Computer von selbst keinen echten Zufall erzeugen. Entweder ist er Bestandteil der Eingabe des Algorithmus, also von einer externen Quelle, ${ }^{192}$ oder nur sogenannter $»$ Pseudozufall«. Dieser wird von Pseudozufallsgeneratoren erzeugt, die selbst deterministisch, ${ }^{193}$ also vorhersehbar sind. ${ }^{194}$ Als Eingabe erhalten diese Generatoren einen sogenannten »seed«, zu welchem sie (vorhersehbare) Zahlen ausgeben, die aber aus stochastischer Sicht den Anforderungen an Zufall genügen. ${ }^{195}$ Dies gilt natürlich nur dann, wenn der seed bekannt ist. In der Praxis kann man neben der oben genannten externen Zuführung von echt zufälligen Werten auch Werte nehmen, die einer ständigen Änderung unterworfen sind (z.B. die aktuelle Uhrzeit). Diese ändern sich permanent und sind nicht wiederkehrend. Das bedeutet, dass nach wie vor vorhergesagt werden könnte, welche Zahlen ausgegeben werden, wenn der seed bekannt ist. Dennoch erzeugt jede (zeitlich unterschiedliche) Ausführung neue - stochastisch hinreichend zufällige (Pseudo)Zufallszahlen. ${ }^{196}$

\section{Vom Algorithmus zur Computersoftware}

Ist der Algorithmus bekannt, bleibt die Frage, was ihn von einer Computersoftware bzw. einem Computerprogramm unterscheidet. Allgemein umschrieben ist ein Computerprogramm eine Folge von Anweisungen, die von einem Computer verstanden wird und - bei vorhandener Eingabe - automatisiert abgearbeitet werden kann. ${ }^{197}$ Es ist somit die konkrete Implementierung eines Algorithmus oder einer Folge von Algorithmen, die miteinander interagieren ${ }^{198}$ und selbst wieder einen (komplexeren) Algorithmus darstellen. ${ }^{199}$ Einen bestehenden Algorithmus kann man aber in

192 Beispielsweise atmosphärisches Rauschen oder zufällige Nutzereingaben.

193 S. u. zum Begriff »Determinismus« in der Informatik, Determinismus, Determiniertheit und Terminierung in der Informatik (S. 59).

194 Karpfinger/Kiechle, S. 25.

195 Vgl. Karloff, Journal of the Association for Computing Machinery 40 (1993), $454 \mathrm{ff}$.

196 Kocarev/Jakimoski/Tasev, LNCIS 292 (2003), 247 ff.

197 Ähnlich auch § 1 (i) der WIPO Mustervorschriften für den Schutz von Computersoftware = GRUR Int. 1978, 286, 290.

198 Vgl. Kindermann, ZUM 1985, 2, 5 f.

199 Haberstumpf, in: Lehmann, Rn. 25. 
verschiedener Weise implementieren, ${ }^{200}$ der Algorithmus gibt die Art nicht vor. ${ }^{201}$ Daraus folgt aber nicht, dass jedes Computerprogramm ein Algorithmus ist, ${ }^{202}$ sondern nur, dass die Gesamtheit von Anweisungen als Algorithmus gesehen werden kann. Zusätzlich enthalten Computerprogramme aber noch die Definition von Datenstrukturen sowie weitere statische Daten, die zum Ablauf notwendig sind (z.B. Konstanten). ${ }^{203}$ Auch der Umkehrschluss, dass jeder Algorithmus ein Computerprogramm ist, gilt nicht, da Algorithmen eben nicht immer automatisiert ausgeführt werden können. Einerseits sind - wie dargelegt - nicht alle Algorithmen für Computer bzw. Maschinen entworfen, andererseits können Anweisungen teilweise noch so abstrakt sein, dass eine Konkretisierung im Rahmen der konkreten Implementierung notwendig ist oder gar gewisse Anweisungen mit spezifischeren Algorithmen ersetzt werden müssen. ${ }^{204}$ Beispielsweise kann in einem Algorithmus festgelegt werden, dass eine Liste sortiert werden muss, der konkrete Sortier-Algorithmus bleibt offen und der Wahl des Entwicklers bzw. Programmierers überlassen. ${ }^{205}$ Freilich können in einem Algorithmus, der ein berechenbares Problem löst, alle abstrakt gehaltenen Stellen durch weitere (konkretere) Algorithmen oder sonstige relevante Daten ersetzt werden und zwar so lange, bis der Algorithmus konkret genug ist, um auf einer Maschine ausgeführt werden zu können. Dieser Prozess ist die sog. Implementierung, bei welcher Auswahlmöglichkeiten bestehen. Dann hat man keinen abstrakten Algorithmus

200 Hiermit ist nicht die Wahl einer Programmiersprache gemeint, selbst in Maschinensprache - die praktisch von niemandem als Implementierungslösung herangezogen wird - sind mehrere Möglichkeiten der Implementierung denkbar.

201 Insbesondere ist auch der sog. »Pseudocode«, mit welchem Algorithmen dargestellt werden können, nicht maschinenausführbar, Cormen/Leiserson/Rivest/ Stein, S. 17 f. Daher ist er weniger nah am maschinenausführbaren Programmcode als die Darstellung zunächst vermuten lässt, so wohl Söbbing, CR 2020, 223, 226.

202 Schricker/Loewenheim/Spindler, §69a UrhG Rn. 12; Dreier/Schulze/Dreier, $\S$ 69a UrhG Rn. 22; Marly, Rn. 31.

203 Güting, S. 1 f.; so wohl auch Kindermann, ZUM 1985, 2, 6.

204 Vgl. Sommerville, S. $106 \mathrm{ff}$.

205 Bei Sortieralgorithmen - die ein typisches Problem in der theoretischen Informatik darstellen - bestehen erhebliche Unterschiede in der Laufzeit. vgl. Cormen/Leiserson/Rivest/Stein, S. 147 ff. 
mehr, sondern ein (konkretes) Computerprogramm. ${ }^{206}$ Somit sind Algorithmen nur das abstrakte - aber hinreichend genaue - Konzept hinter einem Programm. ${ }^{207}$

IV. Folgen für das Wesen der Künstlichen Intelligenz

Ein Modell an sich ist nicht in der Lage, ein konkretes Problem zu lösen, sondern ist nur ein Ansatz für eine Problemlösung. Insoweit sind auch die bisher vorgestellten KI-Methoden nur Modelle zur Lösung von abstrakten Problemen, reichen aber in ihrer Beschreibung nicht aus, um ein konkretes Problem zu lösen. Genauer sind hier Algorithmen, die - auf Computer bezogen - als Rechenvorschriften aus einer Eingabe eine bestimmte Ausgabe erzeugen. In den verschiedenen KI-Methoden kommen bestimmte Algorithmen vor, die allerdings für spezifische Probleme entworfen werden müssen, beispielsweise Trainingsalgorithmen für neuronale Netze. Für die reale Anwendung müssen die KI-Methoden und damit auch die Algorithmen allerdings in Computersoftware implementiert werden. Hierbei gibt das System »Computer« die Restriktionen vor. Daher ist jede real existierende $^{208}$ und damit implementierte Künstliche Intelligenz den gleichen Restriktionen unterworfen wie jeder (andere) Algorithmus und damit jedes andere Computerprogramm. Dies gilt insbesondere bei der Verwendung von Zufall, der entweder Eingabe sein muss oder nur Pseudozufall ist.

206 In diesem Sinne sind wohl auch die Definitionen von Loewenheim/Spindler, Dreier und Marly (alle Fn. 202) zu verstehen, die damit einen zu engen Begriff zugrunde legen.

207 Güting, S. 28; Manber, S. 1; Wirth, S. 7; so wohl auch Haberstumpf, in: Lehmann, Rn. $23 \mathrm{ff}$.

208 Da auch Konzepte und Methoden Entitäten sind, heißt »real existierend « an dieser Stelle, dass die Künstliche Intelligenz in der Lage ist, durch Verhalten eine Zustandsänderung in der realen bzw. physischen Welt zu bewirken, ergo so konkret ist, dass sie als Computersoftware auf irgendeinem Computer ablaufen kann. 


\section{E. Determinismus}

Wie festgestellt, müssen KI-Methoden für eine reale Anwendung in Computerprogramme implementiert werden, die auf Algorithmen basieren. Somit kommt die Frage auf, ob Computerprogramme, damit auch Algorithmen - und letztendlich auch Künstliche Intelligenzen - (immer) deterministisch sind. »Determinismus«, vom lateinischen »determinare«, $\mathrm{zu}$ Deutsch »abgrenzen « ${ }^{209}$ ist ein Homonym, welches einerseits, philosophisch benutzt wird, andererseits auch in der Informatik angewendet wird. Bei der juristischen Wertung von KI-Systemen treffen beide Konzepte unter dem gleichen Begriff aufeinander, sodass differenziert werden muss.

\section{Determinismus und Willensfreiheit in der Philosophie und im Recht}

Im philosophischen Sinne bedeutet Determinismus, dass zukünftige Ereignisse nicht zufällig geschehen, sondern vorherbestimmt sind. Entweder durch einen Gott (sog. »theologischer Determinismus«) oder als Kausalfolge des bereits Geschehenen (sog. »kausaler Determinismus «). ${ }^{210}$ Sofern dies für alle zukünftigen Ereignisse gilt, heißt dies, dass es keinen Zufall gibt, daher auch keine freien Entscheidungen von Subjekten. In einer deterministischen Welt wäre der Wille nicht frei, sondern die zukünftigen Handlungen stünden bereits fest. Somit ist die Frage nach der Willensfreiheit mit der des Determinismus verbunden. ${ }^{211}$ Das Resultat dieses Gedankengangs wurde - als Folge der bisherigen Ergebnisse der Neurowissenschaften - vor allem im Strafrecht diskutiert. Sofern der Wille nicht frei ist, kann es auch keine Schuld geben. ${ }^{212}$ Im zivilrechtlichen Schrifttum wurde diese Problematik seltener diskutiert, spielt aber bei Fragen der Haftung und der Privatautonomie eine Rolle, ${ }^{213}$ worauf noch zurückzukommen ist.

209 Mittelstraß/Mainzer: Determinismus, Bd. 2, S. 167.

210 Sandkühler/Stekeler-Weithofer: Determinismus/Indeterminismus, Bd. 1, S. 382.

211 Mittelstraß/Mainzer: Determinismus, Bd. 2, S. 168.

212 Vgl. Czerner, ArchKrim 218 (2006), 65 ff. m.w.N.; kritisch Stübinger, S. 341 ff.

213 Laufs, MedR 2011, 1, 4 ff.; vgl. auch Waldstein, in: FS Schwind, 1978, 329 ff. 
II. Determinismus, Determiniertheit und Terminierung in der Informatik

Auch in der Informatik findet sich der Begriff »Determinismus «; zusätzlich bestehen zwei weitere verwandte Begriffe, die für das Verständnis von Relevanz sind: »Determiniertheit« und »Terminierung«. Der Begriff »Determinismus « kommt vor allem bei zwei Teildisziplinen vor: Der Automatentheorie und der Kryptologie. In beiden Disziplinen wird der Begriff mit unterschiedlichem Hintergrund verwendet, beschreibt aber dasselbe Phänomen. Grundsätzlich bedeutet Determinismus, dass ein (implementierter) Algorithmus ${ }^{214}$ stets dieselben Schritte und Zwischenergebnisse durchläuft, egal wann und wie oft er ausgeführt wird. ${ }^{215}$ Analog einem Autofahrer, der sein Ziel immer über dieselbe Strecke erreicht. Umleitungen sind ihm ebenso fremd wie alternative Routen. Im Umkehrschluss wäre ein nicht-deterministischer Algorithmus, ein solcher, der eine Vielzahl von unterschiedlichen Schrittfolgen durchläuft. Der konkrete Zustand und damit auch Zwischenergebnisse hängen dabei nicht nur von der Eingabe, sondern vom Zufall ab. Entsprechend würde einem nicht-deterministischen Autofahrer das gesamte Straßennetz zur Verfügung stehen, um zu irgendeinem Ziel zu gelangen, sodass unterschiedliche Strecken denkbar sind. Allerdings handelt es sich bei den nicht-deterministischen Algorithmen um eine theoretische Betrachtung, bei welcher Zufall in Computern als gegeben angenommen wird. Tatsächlich handelt es sich bei diesen Algorithmen um die bereits erwähnten »randomisierten Algorithmen«, die entweder keinen echten Zufall, sondern - deterministischen - Pseudozufall verwenden oder echten Zufall als Eingabe. Gleiches gilt für stochastische Entscheidungen in Algorithmen, da diese bei stets gleicher Eingabe die gleiche Ausgabe erzeugen.

Neben dem Determinismus-Begriff existiert in der Informatik auch das Konzept der Determiniertheit. Ein Algorithmus ist determiniert, wenn er bei einem definierten Eingabewert stets zum selben Ergebnis führt. ${ }^{216}$ Bezogen auf den Autofahrer bedeutet das, dass er stets zu demselben Ort fährt, aber die Route keine Rolle spielt. Bei einem nicht-determinierten Algorithmus sind hingegen der Weg und das Ergebnis ungewiss. Daraus folgt, dass jeder Algorithmus und damit auch jedes Programm bzw. jede Software, die deterministisch ist, auch determiniert ist.

214 Genau genommen ein Automat, der immer dieselben Zustandsübergänge durchläuft.

215 Hromkovič, S. $108 \mathrm{ff}$.

216 Priese/Erk, S. 68. 
Der letzte Begriff ist die Terminierung eines Algorithmus, also dass dieser zu einem Ende kommt. ${ }^{217}$ Es existieren zahlreiche Algorithmen, welche ohne einen Eingriff des Benutzers nie beendet werden. Es ist relativ einfach, ein Programm zu entwerfen, welches in eine Endlosschleife gerät, sich also in einem sich ständig wiederholenden - niemals endenden - $\mathrm{Zu}-$ standszyklus befindet und somit auf einem nicht-terminierten Algorithmus basiert. ${ }^{218}$ Es gibt aber keinen Algorithmus, der für alle (formalen) Algorithmen und damit für alle Programme erkennen kann, ob sie terminieren. Dies wird auch als »Halteproblem « bezeichnet. ${ }^{219}$ Sofern ein reales Programm aber terminiert, ist es den vorherigen Ausführungen nach auch determiniert und deterministisch, sowohl im informatischen als auch im philosophischen Sinne. Aus diesem Grund kann es keine realen, durch Programme und damit in Software implementierten, nicht-deterministischen Algorithmen geben, die terminieren. Da den Darlegungen nach auch jede implementierte Künstliche Intelligenz Computersoftware ist, gilt die hier beschriebene Folgerung uneingeschränkt auch für jede implementierte Künstliche Intelligenz, ${ }^{220}$ konkreter: Jede Künstliche Intelligenz ist deterministisch. $^{221}$

III. Konvergierender bzw. stochastischer Determinismus

Alle real existierenden Programme müssen auf realen Maschinen - also auf einem Computer - ausgeführt werden und unterliegen damit den Restriktionen dieser Maschinen. Hier kann die zur Verfügung stehende Rechenkapazität nicht ausreichen, um für alle möglichen Eingaben sämtliche möglichen Folgezustände zu berechnen. Entweder aufgrund der begrenzten Speicherkapazität oder durch zu lange Rechenzeiten, wodurch das Problem zu komplex wird. Diese Problematik betrifft jegliche Art von Software, die hinreichend komplex ist, und ist daher unabhängig vom Phänomen der Künstlichen Intelligenz. ${ }^{222}$ Allerdings weisen implementierte KI-Methoden eben oft eine entsprechend hohe Komplexität auf, sodass das Problem hier

217 Hoffmann, Grenzen der Mathematik, S. 274.

218 Kurzes Beispiel in Python: »while True: print('BGB')«.

219 Hoffmann, Grenzen der Mathematik, $319 \mathrm{ff}$.

220 Ebenso wohl auch Hoeren/Niehoff, RW 2018, 47, 49.

221 Ebenso wohl auch Käde/v. Maltzan, CR 2020, 66, 67.

222 Auch sind wohl gängige Programme (z.B. Betriebssysteme) für Endbenutzer so komplex, dass auch nicht mehr alle möglichen Eingaben vorherberechnet werden können. 
in besonderer Weise zutage tritt. Das betrifft auch die Algorithmen, die beim Maschine Learning im Lern- bzw. Trainingsprogramm genutzt werden. Um ein gegebenes Problem ressourceneffizient zu lösen, kann der erwähnte Pseudozufall, aber auch ein extern zugeführter, echter Zufall hilfreich sein. ${ }^{223}$ Hierbei wird mit einer zufälligen (schlechten) Lösung begonnen und durch sukzessive kleine Veränderungen das KI-System immer weiter verbessert. Diese KI-Systeme konvergieren üblicherweise gegen eine globale Ideallösung. Der Lern- bzw. Trainingsprozess beginnt dabei aber mit zufällig gewählten Parametern. Nähme man echte Zufallszahlen, wäre der zugrunde liegende Algorithmus zwar gem. der Automatentheorie ein nicht-deterministischer, aber eben nur, weil die Zufallszahlen nicht als Eingabe gewertet werden, was sie in der realen Umsetzung jedoch sind. Verfehlt wäre es deshalb, hierin die Unberechenbarkeit einer Maschine oder gar das Potenzial eines »freien Willen« zu interpretieren. Komplexe, sich selbst optimierende Algorithmen gleichen mehr Bergsteigern, die denselben Gipfel über unterschiedliche Routen erklimmen. Es wird im Rahmen der Optimierung stets gegen das Optimum - den Gipfel - gestrebt. Möglich ist es mitunter, statistische Aussagen über das Verhalten komplexer Programme in vertretbarer Zeit zu erhalten, die entsprechende Tendenzen erkennen lassen. ${ }^{224}$ Dies würde man dann stochastischen Determinismus nennen. ${ }^{225}$ Für neuronale Netze bedeutet das, dass während der Anwendung von diesen, deren Ergebnis in keiner Weise weniger vorhersehbar ist, als das anderer (komplexer) Programme oder gar eines Taschenrechners, der zwei Zahlen addiert. Trainingssysteme beruhen zwar auf Zufallszahlen, was aber nichts am deterministischen Charakter ändert. Potential für eine freie Entscheidung besteht auch hier in letzter Konsequenz nicht. 


\section{F. Fehlerhaftigkeit, Erklärbarkeit und Verhaltensvorhersehbarkeit}

Für einfache Computerprogramme gilt bereits, dass diese nicht fehlerfrei sind. ${ }^{226}$ Gerade im Leistungsstörungsrecht bei Computersoftware wurde dieses Problem ausgiebig diskutiert. ${ }^{227}$ Wenn sich (implementierte) Künstliche Intelligenz aufgrund ihrer deterministischen Eigenschaft nicht von anderer Computersoftware unterscheidet, gilt die Annahme der Fehlerhaftigkeit erst recht für Künstliche Intelligenz. Allerdings können aufgrund der dargelegten steigenden Komplexität, dem Einfluss des Pseudozufalls und der scheinbar stärker werdenden Autonomie mancher Künstlicher Intelligenzen einerseits weitreichende Folgen resultieren und andererseits mag es wirken, als würde sich Künstliche Intelligenz beliebig verhalten.

Im Rahmen der unterschiedlichen Aufgaben, die mit Künstlicher Intelligenz gelöst werden können, ist ein neuronales Netz zur Klassifikation (sog. »Klassifikator«) am anschaulichsten. Hierbei handelt es sich um eine Künstliche Intelligenz, welche eine Eingabe einer bestimmten Klasse zuordnet, beispielsweise um Objekte auf Bildern zu bestimmen (z.B. Fußgänger oder Objekte beim autonomen Fahren). Die Menge an Variablen innerhalb eines solchen neuronalen Netzes ist für einen Menschen allerdings schwer erfassbar. Beispielsweise hat das Bilderkennungsnetz »NASNET « bereits knapp 90 Millionen Variablen. ${ }^{228}$ Diese Netze werden grundsätzlich mit Trainingsdaten trainiert. Diese enthalten eine große Zahl von Beispieldaten, welche den später zu verarbeitenden Daten möglichst nahekommen. Nun werden im Rahmen des Trainings die Gewichte des Netzes unter Einfluss von Pseudozufall so optimiert, dass für möglichst alle Bilder der korrekte Output generiert wird. Ziel ist es, dass die aus dem Training resultierenden Gewichte auch für neue, unbekannte Bilder funktionieren. Diese Annahme kann aber nicht verifiziert werden, da die Menge der möglichen Bilder praktisch unbegrenzt ist. ${ }^{229}$

Es besteht aber die Möglichkeit, einen Teil der Trainingsdaten zurückzuhalten und das Netz mit diesen unbekannten Daten zu erproben (sog. »Evaluation«). Diese Evaluation gibt stichprobenartig Aufschluss, ob das Netz Muster erkennen und zuordnen kann oder ob das Netz die Daten nur »auswendig gelernt « hat. ${ }^{230}$ Eine solche Evaluation ist im übertragenen

226 Heussen, CR 2004, 1, 1 ff.

227 Vgl. Marly, Rn. 1437 ff.

228 Zoph/Vasudevan/Shlens/Le, S. $1 \mathrm{ff}$.

229 Vgl. Sun/Huang/Kroening et al., S. 2.

230 In diesem Fall wird dann von »Overfitting« gesprochen, s. o., Trainingsphase (S. 47). 
Sinne jedoch stets nur ein Indiz und kein Beweis. ${ }^{231}$ Zudem haben Untersuchungen mehrfach gezeigt, dass es recht einfach möglich ist, bei neuronalen Netzen zur Bilderkennung eine falsche Ausgabe zu erzeugen. Die Verfahren für solche sog. "Adverserial Attacks« reichen von dem Generieren von non-sense Bildern ${ }^{232}$ über das Nutzen von gezieltem Rauschen $^{233}$ bis hin $\mathrm{zu}$ simplen Lageänderungen eines Objekts auf einem Bild. ${ }^{234}$ Auch kann sich aufgrund der Mächtigkeit und Komplexität der Netze unbemerkt eine ungewollte Verhaltensweise in das KI-System eintrainieren.

Ein prägnantes Beispiel ist der sog. »Wolf-Husky Klassifikator«.235 Dieser Klassifikator wurde - zu Anschauungszwecken der Problematik anhand eines unausgewogenen Datensets trainiert. ${ }^{236}$ Das Trainingsdatenset enthielt Bilder sowohl von Huskys als auch von Wölfen, wobei die Huskys auf den Trainingsbildern stets in einer grünen Umgebung abgebildet waren, während die Wölfe dagegen stets mit Schnee im Hintergrund. Der so trainierte Klassifikator war im Rahmen der Evaluation - welche ebenfalls mit einem Teil desselben unausgewogenen Datensets durchgeführt wurde in der Lage, die Bilder korrekt zuzuordnen. Allerdings unterschied der Klassifikator nicht zwischen Wölfen und Huskys, sondern orientierte sich unerkannt an Schnee und Gras, womit er eigentlich ein »Schnee-Gras Klassifikator« ist. $^{237}$ Wäre dieser Klassifikator von einem durchschnittlichen Softwareentwickler entwickelt worden, wäre dieser auch mit der üblichen Evaluation nicht in der Lage gewesen, zu erkennen, dass das neuronale Netz nicht in gewollter Weise agiert und damit die eigentliche Aufgabe - das Unterscheiden von Wölfen und Huskys - nicht erfüllt.

Hier wird deutlich, dass auch ein deterministisch ablaufender Trainingsprozess, zumindest aus Sicht eines Dritten, zu subjektiv unvorhergesehenen Ergebnissen führen kann. Das trainierte Netz hat während des Trainingsprozesses letzten Endes lediglich Muster und Strukturen gefunden, die das falsche Problem optimal lösen. Beispiele wie der Wolf-Husky Klassifikator zeigen, dass eine Aussage über die Funktionstüchtigkeit einer Künstlichen

231 Vgl. Hagan/Demuth/Beale/De Jesús, Kap. 22, 27.

232 Nguyen/Yosinki/Clune, S. 3 ff.

233 Karmon/Zoran/Goldberg, S. 4 ff.

234 Alcorn/Li/Gong et al., S. 1.

235 Ribeiro/Singh/Guestrin, S. 8 f.

236 Das Wort »unausgewogen« trifft den englischen Begriff »baised« nur zum Teil. Grundsätzlich sind dies Datensets, welche durch ihre besondere Beschaffenheit fehlerhafte Schlüsse zulassen.

237 Ein anderes Beispiel ist ein System, welches Schiffe erkennen sollte, allerdings eigentlich Wasser erkannt hat; vgl. Käde/v. Maltzan, CR 2020, 66. 
Intelligenz durch eine klassische Evaluation nicht möglich ist. Dieser Umstand erstreckt sich nicht nur auf neuronale Netze, sondern grundsätzlich auf jede komplexere Methode der Künstlichen Intelligenz. Daher stellt sich zunächst die Frage, ob jedes Mal, wenn eine Künstliche Intelligenz eine falsche Ausgabe erzeugt, also ein falsches Verhalten zeigt, dies als Fehler zu bewerten ist und weiterhin ob und wie (falsches) Verhalten im Vorhinein erkannt werden kann.

\section{Vom Sein und Sollen}

Aus der deterministischen Eigenschaft von Künstlicher Intelligenz folgt zwangsläufig, dass eine falsche Ausgabe - sofern sie existiert - bei gleichbleibender Eingabe immer auftritt. Gleichermaßen muss - wie dargelegt die Existenz einer solchen falschen Ausgabe dem Nutzer nicht zwangsweise (negativ) auffallen. Auch kann die passende Eingabe nie auftreten. Zudem kann - falls Zufallszahlen eine Rolle spielen - der eigentlich deterministische Pseudozufall von der Zeit abhängig sein, sodass zu unterschiedlichen Zeiten unterschiedliche Ausgaben entstehen können. ${ }^{238}$ Insoweit ist es notwendig, beim Verhalten einer Künstlichen Intelligenz zwischen dem Sein und dem Sollen zu unterscheiden. Das Sein ist das tatsächliche Verhalten einer Künstlichen Intelligenz, also die Reaktion auf alle (kombinatorisch) möglichen Eingaben. Das Sollen dagegen ist das Verhalten einer Künstlichen Intelligenz, welches sie zeigen soll. Für die Evaluation des Sollens kann nur ein sehr kleiner Bruchteil aller kombinatorisch möglichen Eingaben betrachtet werden, das heißt, das evaluierte Sollen kann nur einen kleinen Teil des Seins abdecken. Bei den dabei verwendeten Eingaben handelt es sich um bekannte und für möglich erachtete Eingaben. Eine vollständige Evaluation aller kombinatorischen Eingaben ist in vertretbarer Zeit nicht möglich. Folglich deckt sich das bekannte Soll-Verhalten nur in einem sehr kleinen Teil mit dem Sein-Verhalten. Welche Eingaben das Soll-Verhalten bestimmen und in welchem Umfang diese zu prüfen sind, kann sich nur im Einzelfall und am Stand der Technik bestimmen und wird im Regelfall danach zu beurteilen sein, welche Eingaben wahrscheinlich sind. Aus den Ausgaben der wahrscheinlichen Eingaben können sodann statistische Aussagen über das Verhalten der Künstlichen Intelligenz getroffen werden. Daraus resultierend, muss - auch für die weitere juristische Betrachtung zwischen einem Fehler und dem weiteren unvorhersehbaren Verhalten

238 S. o., Randomisierte Algorithmen und Pseudozufall (S. 54). 
differenziert werden: Weicht das Sein-Verhalten vom Soll-Verhalten ab, liegt ein Fehler vor. Tritt allerdings ein Verhalten auf der Seite des Seins auf, das auf der Seite des Sollens vorher unbekannt war, so handelt es sich um unvorhersehbares Verhalten. Gewiss ist ein unvorhersehbares Verhalten nach dem ersten (wahrnehmbaren) Auftreten bekannt und muss daher ex post dem Sollen zugeordnet werden, sodass dessen Vermeidung bzw. die Evaluation dieses Verhaltens zukünftig zum Stand der Technik gehört und jedes weitere Auftreten als Fehler zu klassifizieren wäre.

\section{Explainable AI Ansätze}

Der Umstand, dass durch die Evaluation weitreichendes Verhalten nicht erkannt werden kann, kann als ungenügend empfunden werden. ${ }^{239}$ Allgemein gilt, dass ein Produkt - insbesondere, wenn es sicherheitskritisch ist - bei Inverkehrbringen hinreichend getestet und verstanden worden sein sollte. Bei einer Künstlichen Intelligenz, deren Antworten zwar richtig, aber für niemanden vollends nachvollziehbar sind, ist diese Grundanforderung zunächst nicht erfüllt. Es ist auch problematisch, dass der Umstand der fehlenden Erklärbarkeit kaum Rückschlüsse auf die Qualität des KISystems erlaubt. In sicherheitskritischen Anwendungen kann dies zu einem erheblichen Hemmnis bei der Anwendung von Künstlicher Intelligenz führen.

Um diesem Problem zu begegnen, hat sich im Rahmender KI-Forschung das Forschungsfeld der »Explainable AI« oder kurz xAI gebildet. $\mathrm{Zu}$ den wichtigsten Methoden dieses Forschungsgebiets, ${ }^{240}$ insbesondere im Bereich der neuronalen Netze, zählen die Sensitivitätsanalyse (SA), ${ }^{241}$ die Layer-wise Relevance Propagation (LRP) ${ }^{242}$ sowie das Class Activation Mapping (CAM) ${ }^{243}$ Zudem existieren Methoden, um die kaum interpretierbaren, neuronalen Netze in leichter verständliche Entscheidungsbäume zu überführen. ${ }^{244}$ Die detaillierte Funktionsweise dieser Verfahren sollen nachfolgend allerdings nicht näher betrachtet werden. Gemeinsam haben

239 Käde/v. Maltzan, CR 2020, 66 ff.

240 Vgl. Käde/v. Maltzan, CR 2020, 66, 68 ff.

241 Vgl. Baehrens/Schroeter/Harmeling et al., Journal of Machine Learning Research 11 (2010), $1803 \mathrm{ff}$.

242 Vgl. Bach/Binder/Montavon et al., PLoS ONE 10(7):e0130140 (2015), 1 ff.

243 Zhou/Khosla/Lapedriza et al., S. 1 ff.

244 Vgl. Schaaf/Huber/Maucher, S. 1 ff. 
diese Methoden jedoch, dass sie deutlich tiefere Erkenntnisse in die komplexen Prozesse eines neuronalen Netzes zulassen und so das fehlerhafte Verhalten, wie das des Wolf-Husky-Klassifikators, erkennbar machen. Damit wird die dortige Klassifikation von Gras und Schnee zum Fehler, was bei klassischer Evaluation nach der im vorherigen Abschnitt vorgestellten Unterscheidung noch als unvorhersehbares Verhalten gegolten hätte.

Als Einschränkung ist aber zu berücksichtigen, dass jegliche Erklärungsansätze immer lediglich eine Unterstützung bei der Interpretation der Entscheidungen einer Künstlichen Intelligenz sind. Die Methoden erreichen ihre Grenzen, sobald die Erklärung an sich zu kompliziert für einen Entwickler wird, sodass auch hier ein Komplexitätsproblem entstehen kann. Dennoch können nun zahlreiche Fehlerklassen, die bis vor einigen Jahren unerkannt geblieben wären, mit xAI jetzt aufgedeckt werden, sodass Verhalten in diesen Klassen als Fehler und nicht mehr als unvorhersehbares Verhalten betrachtet werden muss. Hierbei ist allerdings zu beachten, dass die hier vorgestellten Methoden den Stand der Forschung beschreiben. Entwicklertools existieren nur vereinzelt ${ }^{245}$ und entsprechende Normen befinden sich derzeit in der Entstehungsphase. ${ }^{246}$ Ungeachtet dessen sind Prinzipen der xAI bereits bekannt und können von einem Entwickler innerhalb weniger Monate implementiert werden. Insofern kann insbesondere bei sicherheitskritischen Anwendungen eine Berücksichtigung dieser Methoden durchaus gefordert werden. ${ }^{247}$

\section{G. Wissen und dessen Repräsentation}

Nach der ausführlichen Darlegung über das Verhalten von Künstlicher Intelligenz steht nun fest, dass eine Künstliche Intelligenz keinen Willen hat, aber handeln und sich verhalten kann. Offen bleibt aber noch, ob eine Künstliche Intelligenz etwas wissen kann und wenn ja, welche Eigenschaften dieses Wissen hat. Gleich den vorangegangenen Begriffen ist auch der Begriff des Wissens domänenabhängig und wird teilweise unterschiedlich betrachtet. In der Informatik wird »Wissen« durch das DIKW-

245 Beispieltools: Bellamy/Dey/Hind et al., S. 1 ff.; Fraunhofer Institut für Nachrichtentechnik, Erklärbarkeit durch LRP, Tool verfügbar unter: https://rpserver.hhi.fraunhofer.de/image-classification (zuletzt abgerufen am 01.06.2020); Google, »What if tool« verfügbar unter: https://pair-code.github.io/what-iftool/fat2020.html (zuletzt abgerufen am 01.06.2020).

246 Vgl. DIN, Künstliche Intelligenz, S. 3.

247 S. u., Fahrlässigkeit (S. 120). 
Modell ${ }^{248}$ beschrieben und von »Zeichen «, »Daten« und »Information « ${ }^{249}$ hierarchisch abgegrenzt. ${ }^{250}$ Das Zeichen ${ }^{251}$ bildet den elementaren Baustein einer formalen Sprache und somit den Anfang der Hierarchie. Werden Zeichen kombiniert, entstehen Daten bzw. Wörter, die auch Entitäten benennen können. Für sich genommen liefern Daten keine Information; um sie zu bilden, müssen sich Daten erst gegenseitig referenzieren. Das erfolgt üblicherweise über einen Subjekt-Prädikat-Objekt-Tripel. Hierbei wird einem Subjekt - sprich einem Konzept, über das eine Aussage getroffen werden soll - mit einem Objekt - also dessen konkreter Ausprägung - verknüpft. Um welche Eigenschaft es sich handelt, wird durch das Prädikat beschrieben. Hierauf aufbauend stellt das Wissen sodann die Vernetzung von Informationen dar. ${ }^{252}$ Die Repräsentation von Wissen in der Informatik, insbesondere in der Lehre der Expertensysteme, ${ }^{253}$ erfolgt üblicherweise durch ein semantisches Netz respektive eine Ressource Description Framework (RDF) Ontologie. ${ }^{254}$ Hierbei können beliebig viele Subjekt-PrädikatObjekt-Tripel verknüpft werden, welche so das semantische Netz bilden. ${ }^{255}$ Inwieweit auch neuronale Netze unter diesen Wissensbegriff fallen, ist zum heutigen Zeitpunkt ungeklärt und Gegenstand der Forschung. ${ }^{256}$

Eine andere Betrachtung von Wissen findet im sog. Wissensmanagement statt. Hier wird Wissen als ein zentraler Wettbewerbsvorteil verstanden, welcher den Erfolg eines Unternehmens maßgeblich mitbestimmt. ${ }^{257}$ Diese Ressource $\mathrm{zu}$ verwalten und $\mathrm{zu}$ erhalten ist das Ziel des Wissensmanagements. ${ }^{258}$ Weite Teile der Begriffswelt der Informatik, wie Daten und Information, finden auch im Rahmen des Wissensmanagements Anwendung. Der Wissensbegriff beschreibt hier alle (Denk-)Modelle über Objekte und Sachverhalte. ${ }^{259}$ Eine Besonderheit im Rahmen des Wissensmanagements ist die Unterscheidung zwischen dem sog. »expliziten

$248 »$ Data, information, knowledge and wisdom «.

249 Gelegentlich wird zudem Weisheit als höchste Stufe der Hierarchie genannt, wobei dies einen theoretischer Ansatz darstellt, der aus Sicht der praktischen Informatik nicht von Relevanz ist.

250 Der Autor hiervon ist unbekannt, vgl. Wallace, S. 13.

251 Beispielsweise A-Z, 0-9 oder Sonderzeichen (+-\# etc.).

252 Vgl. Zech, S. 28 f.

253 S. o., Semantic Web Systeme/Expertensysteme (S. 40).

254 Vgl. Krempel, S. 154.

255 Fischer/Hofer, S. 684 (»RDF«).

256 Vgl. Binder/Bach/Montavon et al., Information Science and Applications 2016, 913 ff.; Shu/Zhu, S. 1 ff.; Yosinski/Clune/Nguyen et al., S. 1 ff.

257 Gronau/Bahrs/Vladova et al., S. 6.

258 Vgl. Wiendahl, S. 328.

259 Wilkesmann/Rascher, S. $19 \mathrm{f}$. 
Wissen« und dem sog. »impliziten Wissen«. Das explizite Wissen stellt hierbei jenen Teil des Wissens dar, welches sich verhältnismäßig leicht auf ein anderes Individuum übertragen lässt. Klassischerweise ist das in Büchern enthaltene Wissen explizites Wissen. Implizites Wissen dagegen ist der Verbalisierung nicht zugänglich ${ }^{260}$ und muss so durch den Lernenden erarbeitet werden. Als Beispiele werden Erfahrungswissen, ${ }^{261}$ motorische Fertigkeiten oder soziales Gespür angeführt. ${ }^{262}$ Der Wissensbegriff im Wissensmanagement dient somit vorrangig dazu, eine Ressource mit ihren Eigenarten zu beschreiben. Im Vergleich ist festzustellen, dass der Wissensbegriff des Wissensmanagements weiter ist als der Wissensbegriff der Informatik. Insbesondere das Konzept des impliziten Wissens unterscheidet sich fundamental von dem Verständnis der Informatik, deren Wissen stets - wenn auch nur maschinell - artikulierbar ist. Auch kann Wissen in Maschinen - mangels Bewusstseins - weder unbewusst noch bewusst vorliegen. Wird also Wissen in Bezug auf Künstliche Intelligenz diskutiert, ist darauf zu achten, die technischen Eigenarten zu berücksichtigen.

\section{H. Zwischenergebnis}

Aus technischer Sicht lässt sich festhalten, dass Künstliche Intelligenz ein Eigenbegriff ist, der zunächst Oberbegriff für diverse (abstrakte) Ansätze (KI-Methoden) darstellt, wie beispielsweise (künstliche) neuronale Netze, Suchbäume, Fuzzylogiken, etc. ${ }^{263}$ Die Methoden an sich sind allerdings nur Konzepte, die in der realen Welt keine Wirkung entfalten. Hierfür bedarf es ihrer Implementierung in Computerprogramme. Daher ist die für die Rechtswissenschaft und damit auch die im weiteren Verlauf relevante Entität die reale Implementierung von Künstlicher Intelligenz in Computerprogrammen. Das Ziel von Künstlicher Intelligenz ist es, Handlungen vorzunehmen, die eigentlich (derzeit) menschliche Handlungen sind. Dieser Zweck wird zwar grundsätzlich mit jedem Computer oder sogar mit jeder Maschine verfolgt, allerdings sucht der Forschungsbereich der Künstlichen Intelligenz nach besonders geeigneten Ansätzen hierfür.

Das Phänomen der Künstlichen Intelligenz ist dabei so alt wie der programmierbare Rechner bzw. der moderne Computer selbst. Schon seit Be-

260 Loenhoff, S. 20.

261 Wiater, S. 22.

262 Loenhoff, S. 4.

263 S. o., Methoden der Künstlichen Intelligenz im Überblick (S. 34). 
stehen solcher Computersysteme wird an derartiger Computersoftware geforscht, was sich gut am Beispiel des Schachcomputers zeigt. Allgemein zeigt Künstliche Intelligenz durch die Handlungen, zu deren Zweck sie geschaffen wurde, ein von außen beobachtbares Verhalten. Aus diesem Verhalten darf aber nicht fehlerhaft geschlussfolgert werden, es würde sich um Maschinen handeln, die denken, also in dem Sinne intelligent sind wie Menschen. Aus den Restriktionen, denen ein Computer unterliegt, folgt deshalb auch, dass die diskutierten Ansätze der sog. »starken Künstlichen Intelligenz« keine Rolle spielen.

Zudem gibt es nicht »die« Künstliche Intelligenz, sondern es handelt sich vielmehr um verschiedene Ansätze zur Lösung bestimmter Probleme mit dafür gezielt geschaffenen, rational und möglichst effizient handelnden Programmen. KI-Methoden basieren auf Algorithmen und sind daher zwangsläufig deren Restriktionen unterworfen. Wie aufgezeigt, sind alle real implementierten Algorithmen, die terminieren, auch deterministisch, sowohl im philosophischen als auch im informatischen Sinne. Für die in der theoretischen Informatik als nicht-deterministisch bezeichneten randomisierten Algorithmen gilt dies ebenfalls, da die für diese Algorithmen notwendigen Zufallszahlen in der Praxis entweder auch deterministisch erzeugt werden oder von der jeweiligen Eingabe abhängen. Aufgrund der steigenden Komplexität von Computerprogrammen im Allgemeinen ist es schon problematisch, dass zwar theoretisch jede Ausgabe zu jeder Eingabe vorherberechnet werden kann, dies aber in der realen Umsetzung viel zu lange dauern würde. Gerade dieses Problem wird durch Künstliche Intelligenz noch einmal verstärkt. Daraus folgt, dass es nicht mehr praktikabel ist - in realistischer Zeit sogar praktisch unmöglich - alle möglichen Handlungsmöglichkeiten vorherzubestimmen, das heißt alle möglichen Eingaben durchzurechnen, um die jeweiligen Ausgaben zu bestimmen. Möglich sind daher nur noch statistische Aussagen. Aus dieser Komplexität kann - jedenfalls nach außen - eine Autonomie von Computerprogrammen bzw. Künstlicher Intelligenz folgen, die sich von rein vorprogrammierten Entscheidungen unterscheidet, ohne aber einen freien Willen der Maschine zu sein. Diese Autonomie ist vielmehr Folge des Anlernens oder einer stochastischen Berechnung der Eingabe. Zumindest theoretisch kann jede Handlung - als Folge des Determinismus - vorherberechnet werden. Dennoch müssen als Konsequenz des zwar deterministischen aber komplex wie komplizierten Phänomens der Künstlichen Intelligenz gewisse rechtliche Ansätze möglicherweise in einem neuen Licht betrachtet werden. Fraglich bleibt daher, ob der Satz von Köhler, »da[ss] auch das komplizierteste Datenverarbeitungssystem letztlich keine autonomen Entscheidungen, 
$\S 2$ Wesen: Willen, Wissen und Verhalten

sondern nur logische Operationen nach einem vorgegebenen Programm durchführen kann«, ${ }^{264}$ letztendlich noch zutrifft.

264 Köhler, AcP 182 (1982), 126, 133. 


\section{§ 3 Zurechenbarkeit und Folgen für das Vertrags- und Deliktsrecht}

Wenn nun Künstliche Intelligenz per se deterministisch ist, allerdings so komplex, dass keine vollständigen Vorhersagen mehr möglich sind, stellt sich die Frage, wie sich auf sie bezogene Erfolge und Tatsachen im Vertrags- und Deliktsrecht auswirken. Eine besondere Relevanz erlangt die Frage dann, wenn Künstliche Intelligenz (scheinbar) autonom entscheidet, also ein sog. »autonomes System《 vorliegt und dessen äußerliche Erscheinung eine rechtliche Folge hat, ${ }^{265}$ insbesondere, ob sich diese Erscheinung und dessen rechtliche Folge von anderen - bisherigen - technischen Phänomen unterscheidet. Im allgemeinen Zivilrecht sind dabei die Rechtsgeschäftslehre und das Haftungsrecht von Interesse. Die weitere Darstellung soll allerdings nicht als umfassende Darlegung der Problematik verstanden werden, sondern vielmehr ausgewählte mögliche Problembereiche im Vertrags- und Deliktsrecht aufzeigen, die bei einem Vergleich mit anderen technischen Phänomenen bisher weniger Relevanz hatten.

Die Auswirkungen auf diese beiden Bereiche sollen im Folgenden anhand der Zurechnungslehre untersucht werden, ${ }^{266}$ wobei sich besonders drängende Fragen für die Zurechnung eines Fehlverhaltens von Künstlicher Intelligenz stellen. Nach einem knappen Überblick über die Zurechnungslehre (A.) folgt zunächst eine Darlegung der beteiligten Entitäten unter Berücksichtigung ihrer Eigenschaft als Rechtssubjekt oder -objekt (B.). Sodann wird die Zurechnung von Willenserklärungen untersucht (C.), die zwar unter strenger Betrachtung als eine reine Verhaltenszurechnung aufgefasst werden kann, aber aufgrund des Elements des Willens einer gesonderten Betrachtung bedarf. Im Weiteren werden die Fragen der Zurechnung des Verhaltens von Künstlicher Intelligenz in Bezug auf mögliche Haftungsfragen erörtert (D.). Zuletzt wird noch kurz auf die Wissenszurechnung eingegangen (E.), die allerdings in Folge der bereits dargelegten eingeschränkten Wissensfähigkeit einer Künstlichen Intelligenz keiner vertieften Betrachtung bedarf. 


\section{A. Grundlagen der Zurechnungslehre}

Grundlegend ist die Frage der Zurechnung insbesondere bei der Wissenszurechnung der juristischen Personen diskutiert worden, ${ }^{267}$ sie spielt aber auch in der Rechtsgeschäftslehre oder im Haftungsrecht eine Rolle. ${ }^{268}$ Abstrakt gesprochen wird bei der Zurechnung eine Tatsache oder ein Erfolg bezogen auf eine Entität hinsichtlich der Rechtsfolgen in die Verantwortung einer Person gestellt. ${ }^{269}$ Es sind somit drei Elemente notwendig: Eine verantwortliche Person (Zurechnungsendsubjekt ${ }^{270}$ ), eine Tatsache oder ein Erfolg (Zurechnungsgegenstand) und eine damit verbundene Entität (Zurechnungsausgangssubjekt). ${ }^{271}$ Stimmen Zurechnungsausgangssubjekt und Zurechnungsendsubjekt überein, so handelt es sich um eine Eigenzurechnung, andernfalls um Fremd- oder Drittzurechnung. Die Zurechnung erfolgt in der Regel über die Zurechnungsvorschriften der $\S \S 31,166,278$ BGB ${ }^{272}$ Üblicherweise kommt der Eigenzurechnung in der Zurechnungslehre wenig Relevanz zu; weil es sich um Konstellationen ohne genuine Zurechnungsprobleme handelt, die in der Zurechnungslehre trivial sind. Dennoch wird bei der weiteren Untersuchung der jeweiligen Zurechnungsgegenstände an der Terminologie der Zurechnungslehre festgehalten, auch wenn es sich bei dem betrachteten Problem herausstellen sollte, dass es sich um Fälle der Eigenzurechnung handelt.

\section{B. $\quad$ Von beteiligten Rechtssubjekten und-objekten}

Begrifflich findet sich bei der Zurechnung des Gegenstandes von einer Entität zu einer anderen Entität in der Zurechnungslehre das Suffix »Subjekt«. Unklar ist zunächst, welche (rechtliche) Qualität diese Subjekte aufweisen müssen. Genauer, ob aus dem Suffix zwangsweise folgt, dass sie Rechtssubjekt sein müssen oder ob nicht zumindest das Zurechnungsausgangssubjekt auch Rechtsobjekt sein kann, da auch Eigenschaften wie das Verhalten zugerechnet werden können, was nicht auf Rechtssubjekte

267 Vgl. Grigoleit, ZHR 181 (2017), 160 ff.; Medicus, in: Möglichkeiten der Wissenszurechnung, S. 4 ff.; Taupitz, in: Möglichkeiten der Wissenszurechnung, S. 16 ff.; Wagner, ZHR 181 (2017), 203 ff.; Buck, S. 104 ff.

268 Vgl. Buck, S. 105.

269 Buck, S. 105; Deutsch, VersR 1988, 1197.

270 Auch Zurechnungsobjekt genannt, vgl. Wolff, S. 248 f.

271 Hacker, RW 2018, 243, 245; vgl. BGH, U. v. 08.12.1989 - V ZR 246/87, NJW 1990, 975, $976=$ BGHZ 109, 327.

272 Buck, S. 105. 
beschränkt ist, sondern durch jede Entität ihren Ausgang nehmen kann. ${ }^{273}$ Auch implementierte Künstliche Intelligenz selbst - als Software - ist de lege lata kein Rechtssubjekt, ${ }^{274}$ sodass die Frage der Qualität des Zurechnungsausgangssubjekt dann eine Bedeutung für diese Untersuchung bekommt, wenn Künstliche Intelligenz dieses Zurechnungsausgangssubjekt ist. Um dieser Frage nachzugehen, muss daher dargelegt werden, welche Entitäten in Bezug auf Künstliche Intelligenz bestehen bzw. beteiligt sind und ob ihnen die Rechtsobjekt- oder Rechtssubjekteigenschaft zukommt. Erst anhand dessen kann dann auch der Frage nachgegangen werden, ob es überhaupt möglich ist, einer Künstlichen Intelligenz de lege ferenda die Eigenschaft des Rechtssubjekts zukommen zu lassen oder ob die Natur der Rechtssubjekteigenschaft dagegen spricht.

\section{Künstliche Intelligenz und auf sie bezogene Entitäten als Rechtsobjekt(e)}

Was Rechtsobjekte sind, ist bis jetzt ungeklärt und streitig, zugleich finden sich die Begriffe »Rechtsgegenstand « oder »Gegenstand «. Ohne vertieft auf den Streitstand einzugehen, ${ }^{275}$ können als Rechtsobjekte mindestens die Entitäten aufgefasst werden, an denen entweder ein absolutes Schutzrecht besteht und damit Herrschaftsrechte über sie ausgeübt werden können oder über die verfügt werden kann, also Verfügungsrechte ausgeübt werden können. ${ }^{276}$ Im bürgerlichen Recht sind dies Sachen oder Rechte. ${ }^{277} \mathrm{Da}$ implementierte ${ }^{278}$ Künstliche Intelligenz Computersoftware ist, kommt ihr mindestens der gleiche Schutz zu wie jeder anderen Software, soweit sie die Voraussetzungen der jeweiligen Schutzrechte erfüllt. Das heißt, es bestehen immaterielle Schutzrechte, die ein absolutes Schutzrecht bieten, zu denken ist hier an den urheberrechtlichen Schutz i.S.d. $\S \S 1,2$ Abs. 1 Nr. 1, 69a Abs. 1 UrhG. ${ }^{279}$

273 S. o., Problematik des Vergleichs zum Menschen (S. 26).

274 Sester/Nitschke, CR 2004, 548, 549 f.; Cornelius, MMR 2002, 353, 354.

275 Vgl. hierzu ausführlich Kreutz, S. 375 ff.

276 Neuner, § 24 Rn. 2 ff.

277 Medicus/Petersen, Rn. 1173.

278 Unimplementierte Künstliche Intelligenz sowie die reinen KI-Methoden sind zunächst nichts weiter als Konzepte oder Ideen, für die allerdings ebenfalls Schutzrechtsansätze denkbar sind.

279 In der Literatur werden mögliche Schutzrechte ausgiebig diskutiert, vgl. Ehinger/ Stiemerling, CR 2018, 761; Hartmann/Prinz, WRP 2018, 1431; Hetmank/Lauber- 
Da Software aber isoliert keinen Zweck erfüllen kann, müssen weitere Entitäten bestehen, die einen Bezug zu ihr haben, üblicherweise in der Form von Hardware. In der Regel sind dies mindestens ein Rechnersystem (Computer) und ggf. weitere Komponenten wie Sensoren oder Aktuatoren. Sensoren sind Komponenten, die Informationen ihrer Umgebung (Helligkeit, Temperatur, Lautstärke, Frequenzen etc.) in ein elektrisches Signal umwandeln, ${ }^{280}$ beispielsweise Kameras oder Mikrofone. Aktuatoren sind das Gegenteil von Sensoren, also Komponenten, bei denen aus elektrischen Signalen und einer Hilfsenergie eine physische Zustandsänderung resultiert, beispielsweise ein Servomotor, aber auch ein Lautsprecher. ${ }^{281}$

Diese weiteren, auf die Künstliche Intelligenz bezogenen Entitäten in der Form von Hardware sind Sachen i.S.d. $\S 90$ BGB. In ihrer Gesamtheit bilden Hard- und Software ein sog. »Computersystem《. Hier bestehen erwähnenswerte - teilweise auch in der juristischen Literatur erwähnte Spezialfälle: Erstens das sog. »Cyber-physische System« (CPS), welches ein Computersystem mit Sensoren und Aktuatoren ist und über ein Computernetzwerk kommuniziert. ${ }^{282}$ Zweitens das »autonome System«, also ein Computersystem mit entsprechend ausgeprägter Autonomie. Das heißt, dass aus den durch die künstliche Intelligenz wahrgenommenen Umweltzuständen ein Verhalten folgt, ohne dass es einer weiteren Interaktion mit dem Menschen bedarf. Drittens der sog. »Roboter $«,{ }^{283}$ bei welchem es sich um eine physische Einrichtung zur Vornahme mechanischer Tätigkeiten handelt. ${ }^{284}$

Auch wenn das Computersystem oft als nur eine Entität wahrgenommen wird, wird aufgrund der möglichen Teilbarkeit des Computersystems in mehrere Entitäten eine Gesamtbetrachtung der rechtstatsächlichen Lage nicht gerecht. Denn es können verschiedene Rechtsobjekte verschiedenen Rechtssubjekten zugeordnet sein. Einerseits durch das sog. »unbundling«, bei welchem Hard- und Software getrennt erworben bzw. vertrieben werden und somit mehrere Hersteller bestehen können. ${ }^{285}$ Andererseits ist auch die Ausführung von Bestandteilen der Künstlichen Intelligenz auf räumlich

Rönsberg, GRUR 2018, 574, 575; Lederer, GRUR-Prax 2019, 152; für Trainingsdaten, Hacker, GRUR 2020, $1025 \mathrm{ff}$.

280 Grote/Feldhusen/Lehr/Göhlich, Dubbel, Kap. I 1.4.1.

281 Fischerauer, in: Rieg/Steinhilper, S. 303 ff.

282 Vgl. Bauernhansl, in: Vogel-Heuser/Bauernhansl/ten Hompel, S. 11 ff.; Rajkumar/

Lee/Sha/Stankovic, in: Design Automation Conference, S. $731 \mathrm{ff}$.

283 Vgl. Zech, in: Gless/Seelmann, S. 168.

284 Vgl. Russell/Norvig, S. 1120.

285 Wagner, in: Faust/Schäfer, S. 26. 
getrennter Hardware möglich. Diese Hardware kann sogar auch noch geteilt werden und dabei verschiedenen Rechtssubjekten zugeordnet sein. Teile der Hardware bestehen dann möglicherweise nur noch aus reinen Sensoren und Aktuatoren. ${ }^{286}$ Infolgedessen können sogar mehrere Benutzer und Betreiber eines Computersystems bestehen.

\section{Beteiligte Rechtssubjekte}

Nachdem geklärt ist, welche Rechtsobjekte existieren können, ist vor einer Untersuchung einer möglichen Eigenschaft von Künstlicher Intelligenz als Rechtssubjekt zu klären, welche (anderen) Rechtssubjekte am Phänomen der Künstlichen Intelligenz beteiligt sind. Allgemein gilt, dass Rechtssubjekt ist, wer Träger von Rechten und Pflichten ist. ${ }^{287}$ Wenn also eine rechtserhebliche Tatsache oder ein rechtserheblicher Erfolg als Zurechnungsgegenstand zugerechnet werden soll, muss das Zurechnungsendsubjekt Träger von Rechten und Pflichten sein und somit Rechtssubjekt. ${ }^{288}$ Da Künstliche Intelligenz kein für sich isoliertes Phänomen ohne Beteiligung Dritter ist, müssen somit (weitere) Rechtssubjekte bestehen, ${ }^{289}$ die daher als Zurechnungsendsubjekt infrage kommen könnten.

Dogmatisch ungeklärt - aber im weiteren Verlauf bedeutsam - ist eine saubere begriffliche Differenzierung zwischen den verschiedenen bestehenden Begriffen: Rechtssubjekt, Rechtsperson und Rechtsfähigkeit. ${ }^{290}$ Wie erwähnt, ist Rechtssubjekt, wer Träger von Rechten und Pflichten ist. Rechtspersonen sind sodann die im ersten Abschnitt des Allgemeinen Teils des BGB genannten Entitäten des Personenrechts, genauer: natürliche und juristische Personen. ${ }^{291}$ Die Fähigkeit, Träger von Rechten und Pflichten $\mathrm{zu}$ sein, nennt man Rechtsfähigkeit. ${ }^{292}$ Folglich ist jedes Rechtssubjekt rechtsfähig und jede rechtsfähige Entität ein Rechtssubjekt. ${ }^{293}$ Fraglich sind sodann zwei Beziehungen: Erstens, ob die Rechts-

286 Vgl. Fn. 280 und 281.

287 BeckOGK/Behme, § 1 BGB Rn. 2; BeckOK/Bamberger, § 1 BGB Rn. 2; MünchKommBGB/Spickhoff, § 1 BGB Rn. 6; Palandt/Ellenberger, vor $§ 1$ BGB Rn. 1; Bork, Rn. $151 \mathrm{ff}$.

288 Buck, S. 108 nennt dies »Zurechnungsfähigkeit«.

289 Wagner, in: Faust/Schäfer, S. 2.

290 Vgl. Schirmer, JZ 2019, $711 \mathrm{ff}$.

291 BeckOK/Bamberger, § 1 BGB Rn. 1.

292 Neuner, § 11 Rn. 1.

293 Bork, Rn. 154. 
fähigkeit nur notwendige oder auch hinreichende Bedingung für das Vorliegen einer Rechtsperson ist. ${ }^{294}$ Zweitens, ob der Begriff der Rechtsperson ein Synonym des Begriffs des Rechtssubjekts ist. ${ }^{295}$ Beide Fragen stehen in einem Zusammenhang: Geht man von einer hinreichenden Bedingung aus, so kann es keine rechtsfähigen Entitäten - also Rechtssubjekte - geben, die keine Rechtspersonen sind. Das hätte zur Folge, dass eine - im nächsten Abschnitt für Künstliche Intelligenz gesondert zu diskutierende - Teilrechtsfähigkeit ${ }^{296}$ nicht möglich wäre. ${ }^{297}$ Die Diskussion um die Rechtsfähigkeit der BGB-Außengesellschaft ${ }^{298}$ zeigt aber, dass diese als Rechtssubjekt zwar rechtsfähig, aber weder natürliche noch juristische Person i.S.d. BGB ist und daher nicht Rechtsperson. ${ }^{299}$ Vielmehr ist sie eine rechtsfähige Personenvereinigung. ${ }^{300}$ Daraus folgt, dass die Menge der Rechtssubjekte nicht auf die Rechtspersonen des BGB begrenzt ist, sondern Rechtssubjekte entweder Rechtspersonen oder weitere rechtsfähige Entitäten sind, ${ }^{301}$ wobei dann zu klären wäre, welchen weiteren Entitäten eine Rechtsfähigkeit zukommen kann und welche Voraussetzungen dafür notwendigerweise erfüllt werden müssen.

Untersucht man die Personenverhältnisse bei den beteiligten Akteuren beim Phänomen der Künstlichen Intelligenz, so sind bereits durch die komplexere Aufteilung der Rechtsobjekte in Trainingsdaten, Hard- und Software schon mehrere Rechtssubjekte denkbar. Hinzu kommt, dass durch mehrere Akteure aufseiten der Benutzung, weitere Mehrparteienverhältnisse möglich sind.$^{302}$ Im Detail sind dies auf der einen Seite Hersteller von Künstlichen Intelligenz, also der Softwarehersteller sowie mögliche weitere Hersteller von Hardware oder von Trainingsdaten, ${ }^{303}$ die im weiteren Verlauf aber zusammenfassend als »Hersteller« bezeichnet werden; auf der

294 So BeckOGK/Behme, § 1 BGB Rn. 3; Klingbeil, AcP 217 (2017), 848, 859 f.; Enneccerus/Nipperdey, S. 477.

295 Beuthien, NJW 2005, 855, 856; Beuthien, JZ 2003, 715, 717; Hattenhauer, JuS 1982, 405, 407; Timm, NJW 1995, 3209, 3210; a.A. Klingbeil, AcP 217 (2017), $848,861 \mathrm{ff}$.

296 Vgl. Wagner, in: Faust/Schäfer, S. 31.

297 Den Begriff ablehnend Klingbeil, AcP 217 (2017), 848, 859 f.; Lehmann, AcP 207 (2007), 225, $237 \mathrm{ff}$.

298 Vgl. BGH, U. v. 29.01.2001 - II ZR 331/00, NJW 2001, 1056 = BGHZ 146, 341; Reuter, AcP 207 (2007), $673 \mathrm{ff}$.

299 Vgl. BeckOK/Schöne, § 705 BGB Rn. 17.

300 MünchKommBGB/Schäfer, § 705 BGB Rn. 253.

301 Ebenso Schirmer, JZ 2019, 711, 715 f.

302 Ebenso Zech, in: Gless/Seelmann, S. 177 f.; Denga, CR 2018, 69, 70.

303 Zwischen dem Hersteller der Software und dem der Trainingsdaten differenziert auch Etzkorn, MMR 2020, 36, 364 f. 
anderen Seite Benutzer. Das sind die Personen, die für eine (ausgelernte ${ }^{304}$ ) Künstliche Intelligenz die Eingaben erzeugen und die Ausgaben entgegennehmen; dies sind typischerweise die Bediener eines Computerprogramms. Für welchen Zweck die Ausgaben verwendet werden, spielt dabei keine Rolle. Denkbar ist auch, dass die Ausgaben - beispielsweise eines evolutionären Algorithmus ${ }^{305}$ - von Herstellern von (anderen) Produkten zur Entwicklung neuer Produkte verwendet werden oder dass Methoden der Künstlichen Intelligenz in der Produktion eingesetzt werden. In diesem Fall sind diese Akteure dann auch Benutzer einer Künstlichen Intelligenz. Sofern die Künstliche Intelligenz nicht durch den Benutzer selbst betrieben wird, gibt es noch gesonderte Betreiber der Künstlichen Intelligenz. Das sind diejenigen, die eine Künstliche Intelligenz auf einer Hardware laufen lassen, die unter ihrer Kontrolle steht und die Künstliche Intelligenz möglicherweise als Dienst - beispielsweise als Clouddienst - zur Verfügung stellen. Das kann so weit gehen, dass beim Benutzer sogar nur noch die Hardware verbleibt, die die notwendigen Sensoren und Aktuatoren beinhaltet, während die eigentliche Künstliche Intelligenz auf den Rechnersystemen des Betreibers abläuft.

III. Rechtssubjektivierung von Künstlicher Intelligenz oder von auf sie bezogenen Entitäten

Wenn nun die Künstliche Intelligenz oder ein autonomes System selbst das Zurechnungsendsubjekt sein soll, muss es - wie in den vorherigen Darlegungen beschrieben - zumindest teilweise rechtsfähig sein, also Träger der Rechte und Pflichten sein, die aus der Zurechnung folgen. ${ }^{306}$ Mindestens eine KI-bezogene Entität wäre somit nicht nur Rechtsobjekt, sondern

304 Bei weiterlernenden Systemen besteht die Besonderheit, dass das System beim Benutzer weiterlernt; je nach Künstlicher Intelligenz kann dies aber so ausgestaltet sein, dass der Benutzer hierauf keinen Einfluss nehmen kann. Andererseits sind auch Künstliche Intelligenzen denkbar, bei welchem der Nutzer den Prozess des Weiterlernens - wie ein Hersteller - beeinflusst. Insoweit können hier im Einzelfall komplexere Konstellationen entstehen, die die Abgrenzung der beteiligten Akteure verwischen können.

305 S. o., Evolutionäre Algorithmen (S. 42).

306 Hacker, RW 2018, 243, 245. 
Rechtssubjekt. ${ }^{307}$ Dann könnten die Zurechnungsgegenstände dem autonomen System selbst zugerechnet werden, sodass eine unproblematische Eigenzurechnung ohne besondere Zurechnungsprobleme entsteht. Allerdings gehen mit einem solchen Ansatz Folgeprobleme einher, die einerseits philosophischer und andererseits juristischer Natur sind. Der in diesem Kontext erstaunlicherweise vorgetragene Vergleich einer Künstlichen Intelligenz mit Sklaven ${ }^{308}$ - die beispielsweise im römischen Recht ebenfalls nicht rechtsfähig waren - ist allerdings verfehlt, denn damit wird vom Sein auf das Sollen geschlossen. Im Gegensatz zu einer Künstlichen Intelligenz hatten Sklaven de facto einen Willen, der darüber hinaus grundsätzlich frei war; Sklaven hatten so zumindest die theoretische Möglichkeit, frei zu handeln, womit sie - wie jeder Mensch - nicht-deterministisch sind. ${ }^{309}$ Dass dieser freie Wille keine relevanten Folgen für die reale Welt hatte, da er in den Grenzen der damaligen Umstände nicht zum Tragen kam, spielt insoweit keine Rolle. Dennoch sollen im Weiteren die philosophischen Probleme - die insbesondere bei einer vollständigen Rechtsfähigkeit der Künstlichen Intelligenz, wie sie einem Menschen zukommt ${ }^{310}$ - im Sinne einer reinen Rechtslehre, ausgespart werden. Vielmehr sollen die juristischen Probleme und Herausforderungen einer Rechtssubjektivierung entsprechender Entitäten herausgearbeitet werden.

Die bisher diskutierten Ansätze sehen vor, einer bisher nicht rechtsfähigen Entität eine teilweise ${ }^{311}$ oder gar vollständige ${ }^{312}$ Rechtsfähigkeit wie einer Rechtsperson zukommen zu lassen. Unter der Bezeichnung »elektronische Person « bzw. »ePerson « wird auch auf europäischer Ebene eine Rechtsfähigkeit von Robotern in der Hinsicht diskutiert, dass sie Haftungssubjekt sein können und für durch sie entstandene Schäden selbst haften. ${ }^{313}$ Fehlgeleitet ist die Diskussion dann, wenn eine Vermenschlichung von Künstlicher Intelligenz stattfindet, insbesondere aufgrund des

307 Nach Bork, Rn. 230 kann eine Entität niemals zugleich Rechtssubjekt und Rechtsobjekt sein, allerdings kommt der juristischen Person ein gewisser »Mischcharakter« zu, Kreutz, S. 50.

308 Harke, in: Gless/Seelmann, S. 97 ff.; Teubner, AcP 218 (2018), 155, 162.

309 In einem anderen Kontext wird das Computerprogramm auch als »vollkommener Sklave« ohne menschliche Eigenschaften und damit auch ohne freien Willen, bezeichnet, vgl. v. Hellfeld, GRUR 1989, 471, 476.

310 Vgl. Wagner, 88 Fordham L.Rev. 591, 595 ff. (2019).

311 Schirmer, JZ 2019, 711, 716 f.; Teubner, AcP 218 (2018), 155, 178 ff.

312 Kersten, JZ 2015, 1, 6 ff.; Mayinger, S. 166.

313 Europäisches Parlament, Zivilrechtliche Regelungen im Bereich Robotik, P8_TA(2017)0051, Rn. 59. 
deterministischen Verhaltens und des fehlenden Willens. Neben den grundrechtlichen Implikationen einer solchen vollständigen Rechtsfähigkeit ${ }^{314}$ spricht vieles dafür, dass für eine volle Rechtsfähigkeit die Fähigkeit von Denken und Wissen vorhanden sein sollte, ${ }^{315}$ die eine Künstliche Intelligenz im geforderten Sinne nicht hat. Zwar verfügt auch eine rechtsfähige juristische Person nicht über diese Fähigkeiten, allerdings werden sie dann von Dritten ausgeübt, die über diese Fähigkeiten verfügen. Gerade bei Künstlicher Intelligenz wird die Rechtsfähigkeit aber deshalb diskutiert, weil die Fähigkeiten nicht bei einem Dritten liegen, sondern angeblich bei ihr selbst; ${ }^{316}$ insoweit würde eine Künstliche Intelligenz einer natürlichen Person eher entsprechen als einer juristischen Person. Da eine solche Nähe aber nicht zutrifft, stehen die Prinzipien der Rechtsperson einer vollen Rechtsfähigkeit im Sinne einer Rechtsperson entgegen. ${ }^{317}$

Geht man hingegen pragmatisch von der Frage aus, ob die Notwendigkeit einer teilweisen Rechtsfähigkeit existiert, bestehen zunächst die klassischen Probleme wie Beginn und Ende der Rechtsfähigkeit, Regelungen zum Verbleib des Vermögens bzw. die Rechtsnachfolge nach Ende der Rechtsfähigkeit sowie die Durchführung von Maßnahmen der Zwangsvollstreckung, ${ }^{318}$ die sich aber gewiss gesetzgeberisch lösen lassen. Schwieriger werden zwei Fragen: Erstens, anhand welcher Tatbestandsmerkmale festgestellt werden kann, ob eine vorliegende Entität die genauer zu definierenden Tatbestandsmerkmale erfüllt, zweitens, auf welche genaue Entität sich eine Teilrechtsfähigkeit beziehen soll. Eine unscharfe Abgrenzung würde hier zu erheblicher Rechtsunsicherheit führen. Fraglich bleibt, ob ein dann (teil-)rechtsfähiges System überhaupt über einen Grad an Autonomie verfügen und wenn ja, wie hoch dieser sein muss. Nicht geklärt ist, ob auf die Software - als eigentliche Künstliche Intelligenz - abgestellt wird, auf die Hardware als ausführende Instanz oder eine Kombination von Soft- und Hardware. Auch ist zu prüfen, wie sich Änderungen der Substanz des Gesamtsystems auswirken, beispielsweise ein Austausch von Hard- oder Software. Unklar ist dann, ob die Teilrechtsfähigkeit der alten Entität endet und eine neue entsteht oder die Teilrechtsfähigkeit gar überspringt. Gänzlich unklar bleibt schließlich, wie damit umzugehen sein wird, wenn die Software von einer bestimmten Hardware auf weitere

314 Vgl. Wagner, in: Faust/Schäfer, S. 30 f.

315 Klingbeil, AcP 217 (2017), 848, $860 \mathrm{f}$.

316 Vgl. Hacker, RW 2018, 243, $251 \mathrm{ff}$.

317 Im Ergebnis ebenso Denga, CR 2018, 69, 77; Teubner, AcP 218 (2018), 155, 162 f.

318 Vgl. Zech, in: Gless/Seelmann, S. 202 f. 
Hardware kopiert wird und danach womöglich mehrere (teil-)rechtsfähige Entitäten bestehen.

Sofern für diese Probleme annehmbare Lösungen gefunden würden, stellt sich dennoch die Frage, ob es sinnvoll ist, bestehende Lücken durch eine Teilrechtsfähigkeit zu schließen. ${ }^{319}$ Auch andere potenziell gefährliche, komplexe und schwer beherrschbare Technologien, wie beispielsweise die Energiegewinnung durch Kernspaltung, führen nicht zu einer Teilrechtsfähigkeit der mit der Technologie verbundenen Entitäten, sondern zu einer Zurechnung zu einer bereits rechtsfähigen Entität. Im Wesen der Künstlichen Intelligenz ist auch kein Anlass zu finden, das zugrunde liegende technische Phänomen anders $\mathrm{zu}$ behandeln als bisherige technische Phänomene. Daher ist für eine pragmatische Betrachtung der Notwendigkeit einer Teilrechtsfähigkeit im weiteren Verlauf zunächst zu untersuchen, ob überhaupt besondere Probleme beim Einsatz von Künstlicher Intelligenz im Vertrags- und Deliktsrecht bestehen und wenn ja, ob sodann eine entsprechende Notwendigkeit der Teilrechtsfähigkeit besteht oder ob möglicherweise einfachere Lösungen dem Problem gleich oder besser begegnen.

\section{Rechtsobjekte als Zurechnungsausgangssubjekt}

Würde man verneinen, dass Künstliche Intelligenz oder eine bezogene Entität Rechtssubjekt sein kann, bleibt die Frage der Rechtsnatur des Zurechnungsausgangssubjekts. Anders als das Zurechnungsendsubjekt - das immer Rechtssubjekt sein muss - muss bei der Fremdzurechnung das Zurechnungsausgangssubjekt nicht zwangsläufig Rechtssubjekt sein. Entgegen dem Suffix »Subjekt« kann das Zurechnungsausgangssubjekt jede verkörperte Entität sein, ${ }^{320}$ sofern der Zurechnungsgegenstand nicht genuin menschlich ist. Dies zeigt sich schon am klassischen Zurechnungsgegenstand des Verhaltens, das seiner Definition nach beschrieben wird als ein Prozess einer verkörperten Entität über die Zeit, verbunden mit einer von außen beobachtbaren Zustandsänderung, ohne dass es auf die Begründung dieser Zustandsänderung ankommt. ${ }^{321}$ Daraus folgt, dass sich auch Entitäten, die nicht Rechtssubjekt sind, verhalten können, beispielsweise Tiere, aber auch jegliche technischen Phänomene, vom Computer über 
Kraftfahrzeuge bis hin zu Kernkraftwerken, ohne dass die Ursache des Verhaltens für das Verhalten selbst von Relevanz ist. Insoweit besteht hinsichtlich der Zurechnungslehre kein Problem der Zurechnung von Gegenständen von Künstlicher Intelligenz, sofern diese Attribute auch auf die Künstliche Intelligenz zutreffen. Bei genuin menschlichen Attributen, ${ }^{322}$ die möglicherweise zugerechnet werden können, muss auch das Zurechnungsausgangssubjekt ein Mensch sein. Das ist allerdings Folge und nicht Voraussetzung, da ein genuin menschliches Attribut bei anderen Entitäten schlicht nicht vorkommen und damit auch nicht zugerechnet werden kann.

\section{Willenserklärungszurechnung}

Bei der Frage der Zurechnung von Tatsachen oder Erfolgen ist die Willenserklärung als das Mittel des Rechtsgeschäfts ${ }^{323}$ von besonderer Relevanz. Wird eine technische Einrichtung für die Erzeugung oder die Übermittlung von Willenserklärungen verwendet, besteht möglicherweise ein Einfluss der technischen Einrichtung auf die Willenserklärung, wodurch Zurechnungsfragen aufkommen. In Bezug auf Künstliche Intelligenz ist ein solcher Einfluss sowohl beim Vertragsschluss als auch bei der Ausübung von Gestaltungsrechten denkbar. ${ }^{324}$ Dabei stellt sich einerseits die Frage, ob und wann eine Willenserklärung vorliegt und andererseits, ob etwaige Willenserklärungen besondere Wirksamkeitsprobleme aufweisen. Die grundsätzliche Problematik ist aufgrund der Rückführung von Künstlicher Intelligenz zu einem Computerprogramm zwar keine neue, allerdings könnte der steigende Grad an Autonomie des Computerprogramms neue Herausforderungen bei Willenserklärungen aufkommen oder alte Fragestellungen und deren diskutierte Lösungen in einem neuen Licht erscheinen lassen. Sieht man die technischen Phänomene als technisches Äquivalent zu den Hilfspersonen, so läge es nahe, bei ansteigender Autonomie das Recht der Stellvertretung anzuwenden, sodass durch die Zuordnungsvorschrift des $\S 164$ Abs. 1 S. 1 BGB Erklärungen eines Computer- oder KI-Systems für und gegen den dann vertretenen Benutzer des Systems wirken. ${ }^{325}$ Voraussetzung wäre aber nicht nur eine teilweise Rechtsfähigkeit

322 Was genau solche Attribute sind, wird an dieser Stelle bewusst offengelassen und stellt eine Frage der philosophischen Anthropologie dar.

323 Medicus/Petersen, Rn. 175.

324 Vgl. Grapentin, S. 86 ff.

325 Specht/Herold, MMR 2018, 40, 43; Teubner, AcP 218 (2018), 155, 182 ff. 
derartiger Systeme, ${ }^{326}$ sondern auch eine eigene Willenserklärung des Systems. Gerade Letzteres wird bei der derzeitigen und noch näher darzulegenden Willenserklärungsdogmatik fraglich sein. ${ }^{327}$

Zwar wird in der Literatur die Willenserklärungsproblematik auch mit dem Argument diskutiert, dass Künstliche Intelligenz nicht-deterministisch sei, ${ }^{328}$ was als Prämisse - wie dargelegt - technisch falsch ist: Die Folgerungen daraus sind aber sowohl durch die steigende Komplexität der Software als auch durch ihren praktischen Einsatz bei Rechtsgeschäften nicht von der Hand zu weisen. Einer Künstlichen Intelligenz können als Computerprogramm zwar aufgrund der deterministischen Eigenschaft die Vorzüge der Privatautonomie nicht zukommen, allerdings ist die bisherige Ansicht, dass Computer keine Entscheidungen treffen können, ${ }^{329}$ überholt.

Um dem Phänomen näher nachzugehen, wird nach einer kurzen Abhandlung der relevanten Probleme der Willenserklärungsdogmatik zunächst untersucht, wie Willenserklärungen und deren Wirksamkeit bei der Verwendung herkömmlicher technischer Einrichtungen zu bewerten sind, wobei sich im Rahmen dessen auf die technischen Einrichtungen beschränkt wird, an welchen Computer beteiligt sind. Sodann werden zwei Szenarien des Einsatzes von Künstlicher Intelligenz bei Rechtsgeschäften untersucht: Einerseits die Verwendung von Künstlicher Intelligenz als Hilfsmittel und andererseits autonome Erklärungen durch Künstliche Intelligenz. ${ }^{330}$ Die dabei dargestellten Anwendungsszenarien sind freilich teilweise einfach gehalten, sodass auch komplexere Szenarien denkbar sind. Dennoch dürften die gewählten Szenarien geeignet sein, die Problembereiche aufzuzeigen und $\mathrm{zu}$ klären, ob die Lösung für elektronische Willenserklärungen bei herkömmlichen technischen Einrichtungen auf die Situation bei Künstlicher Intelligenz - insbesondere bei autonomen Erklärungen - übertragbar ist. ${ }^{331}$

326 S. o., Rechtssubjektivierung von Künstlicher Intelligenz oder von auf sie bezogenen Entitäten (S. 77).

327 Ebenso Specht/Herold, MMR 2018, 40, 43.

328 Teubner, AcP 218 (2018), 155, 174 ff.; Reusch/Weidner, Rn. 44 ff.

329 Medicus/Petersen, Rn. 256; Köhler, AcP 182 (1982), 126, 133; Fritzsche/Malzer, DNotZ 1995, 3, 7; Neuner, JuS 2007, 881.

330 So auch Foerster, ZfPW 2019, 418, $421 \mathrm{ff}$.

331 Vgl. Specht, GRUR 2019, 253, 256. 


\section{Dichotomie von Wille und Erklärung}

Die Willenserklärung besteht - nicht nur begrifflich - aus dem subjektiven Tatbestand des Willens und dem objektiven Tatbestand der Erklärung. ${ }^{332}$ Über die Relevanz dieser beiden Elemente bestand schon vor Inkrafttreten des BGB Uneinigkeit. Die Bedeutung der Frage geht über die reine akademische Bedeutung hinaus und spielt für die Möglichkeit von (Willens-)Erklärungen durch Künstliche Intelligenz eine Rolle. Ohne zu ausführlich auf den Streitstand einzugehen, ${ }^{333}$ sollen die relevanten Eckpunkte kurz dargelegt werden: Bei der insbesondere von Savigny ${ }^{334}$ vertretenen Willenstheorie ${ }^{335}$ besteht eine natürliche Verbindung zwischen dem Willen der Erklärenden und der Erklärung selbst, sodass dem Willen die relevante Bedeutung zukommt und die Erklärung nur als Zeichen der Erkennung für Dritte dient. Eine Erklärung, die nicht dem tatsächlichen Willen entspricht, wäre sodann nichtig. Der Erklärungstheorie nach besteht dagegen ein Interesse des Verkehrs an dem Vertrauen auf die Richtigkeit der Erklärung, unabhängig vom Vorliegen eines entsprechenden Willens. Der Gesetzgeber wollte sich allerdings beim Entwurf des BGB nicht festgelegen. ${ }^{336}$ In den Materialien zum BGB wird bei der Definition des Rechtsgeschäfts aber deutlich, dass eine Rückführung des Erfolges auf den Willen angedacht war: »Rechtsgeschäft im Sinne des Entw[urfs] ist eine Privatwillenserklärung gerichtet auf das Hervorbringen eines rechtlichen Erfolges, der nach der Rechtsordnung deswegen eintritt, weil er gewollt war $«{ }^{337}$ Hierbei wurden die Begriffe $»$ Willenserklärung« und »Rechtsgeschäft« bewusst synonym verwendet. ${ }^{338}$ Später entstand die Geltungstheorie, wonach der Willenserklärung zwei Bedeutungen zukommen: Erstens wird durch die Willenserklärung ein bereits gefasster Wille erklärt, der so Geltung erlangen soll. Daher ist die Willenserklärung Geltungserklärung. Zweitens dient sie auch der Kenntnisnahme durch mindestens einen Dritten, sodass ihr eine soziale Funktion zukommt. ${ }^{339}$

Insoweit bleibt die Grundfrage, welche Voraussetzungen erfüllt sein müssen, damit ein Verhalten als Willenserklärung qualifiziert werden

332 Bork, Rn. 566; Neuner, § 30 Rn. 1.

333 Vgl. hierzu ausführlich Bork, Rn. 582 ff.; Flume, S. 49 ff.; Musielak, AcP 211 (2011), 769, 771 ff.; Zimmermann, AcP 193 (1993), 121, 129 ff.

334 Savigny, S. 258.

335 Auch Willensdogma genannt.

336 Vgl. Mugdan I, $720=$ Prot. I S. 197.

337 Mugdan I, 421 = Mot., 126.

338 Mugdan I, 421 = Mot., 126; kritisch, Flume, S. 25 ff.

339 Neuner, § 30 Rn. 6; Stathopoulos, in: FS Larenz, S. 357 ff. 
kann. ${ }^{340}$ Je nachdem, welche Voraussetzungen als obligatorisch angesehen werden, kann dies gerade bei autonomen Systemen unterschiedliche Ergebnisse hervorbringen. Gemeinhin wird die Willenserklärung in einen objektiven und einen subjektiven Tatbestand unterteilt. Der objektive Tatbestand ist erfüllt, wenn jemand durch ein Erklärungszeichen aus Sicht eines objektiven Dritten durch einen (kundgetanen) Willen derart erklärt, dass eine Rechtsfolge herbeigeführt werden soll. ${ }^{341}$ Der subjektive Tatbestand besteht sodann aus drei Bestandteilen: Dem Handlungswillen (Handlungsbewusstsein), dem Erklärungswillen (Erklärungsbewusstsein) und dem Geschäftswillen (Rechtsfolgewille im engeren Sinne). ${ }^{342}$ Der Handlungswille ist der Wille, überhaupt eine äußere Handlung bewusst durch Verhalten $\mathrm{zu}$ begehen oder zu unterlassen, ${ }^{343}$ wobei durch diese Handlung das Erklärungszeichen gesetzt wird, ${ }^{344}$ also der objektive Tatbestand durch die Handlung erfüllt wird. ${ }^{345}$ Der Erklärungswille bzw. das Erklärungsbewusstsein geht darüber hinaus und beinhaltet, dass dem Erklärenden bewusst ist, dass die Handlung bzw. das Verhalten ein Erklärungszeichen ist, sie somit rechtserheblich ist, also irgendeine rechtliche Folge hat. ${ }^{346}$ Der herrschenden Auffassung nach muss dieses Tatbestandsmerkmal nicht zwingend vorliegen. ${ }^{347}$ Der Erklärende muss aber mit der nach der Verkehrssitte üblichen Sorgfalt, mit Rücksicht auf Treu und Glauben erkennen und vermeiden können, dass seine Handlung als Willenserklärung aufgefasst werden kann, wenn sie durch den Erklärungsempfänger für eine Willenserklärung gehalten wird (sog. Erklärungsfahrlässigkeit). ${ }^{348}$ Unter dem Geschäftswillen wird der Wille verstanden, die konkrete Rechtsfolge herbeizuführen bzw. das konkrete Rechtsgeschäft zu vollführen. ${ }^{349}$ Fallen allerdings die (objektive) Erklärung und der Geschäftswillen auseinander, hindert dies - aufgrund bestehender

340 Musielak, AcP 211 (2011), 769, 777.

341 Vgl. Köhler, § 6 Rn. 2.

342 Bork, Rn. 578 ff.; Flume, S. 45 ff.; kritisch Neuner, § 32 Rn. 1 ff.; Neuner, JuS 2007, 881, $883 \mathrm{ff}$.

343 Vgl. BeckOGK/Rehberg, § 116 BGB Rn. 34.

344 Flume, S. 46.

345 Bork, Rn. 589.

346 Flume, S. 46 f.; Bork, Rn. 593; Medicus/Petersen, Rn. 605; Stadler, § 17 Rn. 8.

347 Str. vgl. kritisch MünchKommBGB/Armbrüster, Vor 1116 BGB Rn. 27; Musielak, AcP 211 (2011), 769, $791 \mathrm{ff}$.

348 St. Rsp. BGH, U. v. 11.06.2010 - V ZR 85/09, NJW 2010, 2873, 2875; BGH, U. v. 14.05.2002 - XI ZR 155/01, NJW 2002, 2325, 2327; BGH, U. v. 02.11 .1989 - IX ZR 197/88, NJW 1990, 454, 456 = BGHZ 109, 171; BGH, U. v. 07.06.1984 - IX ZR 66/83, NJW 1984, 2279, Ls. = BGHZ 91, 324.

349 BeckOGK/Rehberg, § 116 BGB Rn. 51: Flume, S. 47. 
Anfechtungsmöglichkeiten - die Wirksamkeit der Willenserklärung nicht; ${ }^{350}$ zudem wird durch einen Erst-Recht-Schluss mit der Situation des fehlenden Erklärungsbewusstsein argumentiert, dass der Geschäftswillen nicht konstitutiv sein kann. ${ }^{351}$ Im Gesamten sind die subjektiven Merkmale auch nach Inkrafttreten des BGB - insbesondere mit systematischen Argumenten - in die Kritik geraten, vor allem, da $\S \S 105,116$ ff. BGB von einer Willenserklärung ausgehen, ohne dass subjektive Merkmale erfüllt sind. ${ }^{352} \mathrm{Ob}$ ein Verzicht auf die klassischen, subjektiven Merkmale einer Willenserklärung und damit auf das Merkmal des Willens, mit der Willenserklärungsdogmatik vereinbar sind, bleibt offen. ${ }^{353}$

Grundsätzlich gilt die Willenserklärung als Mittel des Rechtsgeschäfts, welches Mittel der Privatautonomie ist. ${ }^{354}$ Die Willenserklärung gibt die Möglichkeit, private Rechtsverhältnisse nach eigenem Willen frei zu gestalten; ${ }^{355}$ dabei wird sowohl von einem freien Willen als auch von einer freien Ausgestaltung der Privatrechtsverhältnisse ausgegangen. ${ }^{356}$ Somit ist sie Folge eines nicht-deterministischen Weltbildes. Eine per se deterministische Künstliche Intelligenz ${ }^{357}$ - die unabhängig vom Grad ihrer Autonomie - keinen (eigenen) Willen hat, wirft hier Probleme auf. Folglich ist nach derzeitigem Verständnis eine Zurechnung der Erklärung zu einer Entität notwendig, die einen Willen haben kann. ${ }^{358}$ Da nicht der Wille der Zurechnungsgegenstand ist, sondern die Erklärung, handelt es sich im strengen Sinne um einen Sonderfall der Verhaltenszurechnung. Dieses Verhalten wirkt sich sodann auch auf die Privatautonomie derer aus, für und gegen die mithilfe von Künstlicher Intelligenz abgeschlossenen Rechtsgeschäfte möglicherweise wirken.

350 Vgl. MünchKommBGB/Armbrüster, Vor $§ 116$ BGB Rn. 22.

351 Bork, Rn. 600.

352 Brehmer, JuS 1986, 440, 443 f.; Kellmann, JuS 1971, 609, 612; Leenen, JuS 2008, 577, 579 ff.; im Ergebnis HKK-BGB/Schermaier, §§ 116-124 BGB Rn. 14.

353 Vgl. Musielak, AcP 211 (2011), 769, 772.

354 Medicus/Petersen, Rn. 175.

355 Neuner, § 2 Rn. 14; vgl. auch Riesenhuber, ZfPW 2018, 352, 355; C. Paulus/Zenker, JuS 2001, $1 \mathrm{ff}$.

356 Neuner, $\S 10$ Rn. 27 ff.

357 S. o., Determinismus (S. 58).

358 Taupitz/Kritter, JuS 1999, 839; Heun, CR 1994, 595; Mehrings, MMR 1998, 30, 31. 


\section{Herkömmliche technische Einrichtungen}

Bei technischen Einrichtungen bestehen in Bezug auf Willenserklärungen grundsätzlich zwei Ansatzpunkte: Einerseits die Verkörperung von Willenserklärungen, andererseits deren Übertragung. Dies gilt insbesondere seit dem Aufkommen der elektronischen Kommunikation. ${ }^{359}$ Eine reine Verkörperung findet insbesondere mit technischen Einrichtungen statt, die eine Niederlegung vereinfachen bzw. erst ermöglichen: Beginnend mit Stift und Papier über die Schreibmaschine hin zum Personal Computer mit Drucker. Nur zur Übertragung dienen technische Einrichtungen der Sprachübertragung wie das (analoge) Telefon oder (teil-)automatisierte Botensysteme (z.B. Rohrpost), wobei bei Letzterem die Verkörperung in einem vorgelagerten Akt durch andere Hilfsmittel stattfindet. Zwar ist zwischen der Verkörperung und der Übertragung zu differenzieren, ${ }^{360}$ dennoch gibt es technische Einrichtungen, die eine Hybridstellung einnehmen, die also neben der Übertragung zugleich auch eine Verkörperung - sei es durch Speicherung oder Druck - vornehmen, wobei die Übertragung und die Verkörperung in einem technischen Zusammenhang stehen. ${ }^{361} \mathrm{Zu}$ denken ist hier an Fernschreiber und Fax-Geräte, die im klassischen Sinne nach der Übertragung die Erklärung durch einen Druckvorgang verkörpern, ${ }^{362}$ vor allem aber an die digitale Übertragung mithilfe von vernetzten Computern.

Bezüglich der Eigenschaft der Verkörperung muss allerdings differenziert werden. Technische Prozesse in Computern ${ }^{363}$ und Netzwerken ${ }^{364}$ erfordern immer eine - zumindest kurzfristige - Speicherung in dem Sinne, dass sich der physische Zustand in einem flüchtigen oder nichtflüchtigen Speicher ändert. Dies hätte zur Folge, dass jegliche (technische) Speicherung als Verkörperung anzusehen wäre und eine mithilfe eines Computers erstellte Willenserklärung dann immer als verkörpert gelten würde, auch wenn die gespeicherte Entität und damit die Erklärung nur einen Bruchteil von Sekunden existiert. Letzteres ist gerade bei der Internet-

359 Vgl. Wiebe, S. 1 ff.

360 Hoeren/Sieber/Holznagel/Kitz, Teil 13.1, Rn. 37, 57.

361 Da dies in der Regel elektronisch geschieht, wird auch von einer »elektronisch übermittelten Willenserklärung « gesprochen, vgl. Fritzsche/Malzer, DNotZ 1995, 3, 8, wobei auch analoge Verfahren - wie der Fernschreiber - bestehen.

362 Die Verkörperung kann aber auch durch Speicherung auf einem Datenträger stattfinden.

363 Vgl. Patterson/Hennessy, S. 443 ff.

364 Vgl. zur Zwischenspeicherung im sog. »Routing«, Tanenbaum/Wetherall, S. $413 \mathrm{ff}$. 
oder Videotelefonie der Fall, ${ }^{365}$ denkbar sind aber auch direkt in das Computersystem eingegebene und abgearbeitete Befehle, die nach der Ausführung nicht mehr im Speicher existieren. ${ }^{366}$ Sinnvoller ist es daher, neben der Speicherung auch eine gewisse Dauerhaftigkeit der Erklärung zu fordern, sodass seitens des Erklärungsempfängers ein nicht zeitgleicher Abruf möglich ist. ${ }^{367}$

Gerade bei den im Folgenden weiter betrachteten Willenserklärungen, an denen ein Computer beteiligt ist, führt die fortschreitende Automatisierung möglicherweise zu Problemen bei der Willenserklärungszurechnung. ${ }^{368} \mathrm{Be}-$ züglich des Anteils, den der Computer an der Willenserklärung hat, wird graduell zwischen der elektronisch übermittelten Willenserklärung, der automatisierten Willenserklärung ${ }^{369}$ und teilweise der Computererklärung ${ }^{370}$ differenziert. Während sich bei einer elektronisch übermittelten Willenserklärung der Mensch nur der Maschine zum Zwecke der Willenskundgabe und -übertragung bedient, sei eine automatisierte Willenserklärung bereits eine automatische - aber dennoch konkret definierte - Reaktion auf Ereignisse, beispielsweise eine automatisierte Annahmeerklärung. ${ }^{371}$ Die Computererklärung soll noch einen Schritt weiter gehen und eine autonome Willenserklärung als Reaktion auf Ereignisse ohne vordefinierte, konkrete, menschliche Erklärung sein. ${ }^{372}$

Grundsätzlich stellt sich die Frage, ob diese Differenzierung trennscharf und geeignet ist. ${ }^{373}$ Teilweise findet sich die Ansicht, dass die unterschiedlichen Erklärungen dennoch gleich zu behandeln seien, da grundsätzlich das Verhalten von Computerprogrammen vorprogrammiert ist und somit keine

365 Ebenso Hoeren/Sieber/Holznagel/Kitz, Teil 13.1 Rn. 63; Greiner/Kalle, JZ 2018, $535,540 \mathrm{f}$.

366 Im Kontext einer Willenserklärung ist hier an eine verzögert durch den Computer abzusendende Erklärung zu denken, wobei der die Verzögerung steuernde Befehl, nachdem die Erklärung in das Computernetzwerk gelangt ist, nicht mehr im Speicher ist.

367 John, AcP 184 (1984), 385, 391; Hoeren/Sieber/Holznagel/Kitz, Teil 13.1 Rn. 63; Herwig, MMR 2001, $145 \mathrm{f}$.

368 Vgl. Janal, AcP 215 (2015), 830, $831 \mathrm{ff}$.

369 MünchKommBGB/Säcker, Einl. BGB Rn. 188 f.; Hoeren/Sieber/Holznagel/Kitz, Teil 13.1, Rn. 37 ff.; Heun, CR 1994, 595; Köhler/Fetzer, Rn. 167 ff.

370 Vgl. D. Paulus, JuS 2019, 960, 962 f.

371 Spindler/Schuster/Spindler, Vorb. zu § 116 ff. BGB Rn. 1.

372 Cornelius, MMR 2002, 353, 354; Mehrings, MMR 1998, 30, 31.

373 Hoeren/Sieber/Holznagel/Kitz, Teil 13.1 Rn. 4. 
gesonderte Behandlung in der Rechtsgeschäftslehre notwendig sei. ${ }^{374}$ Vorprogrammierung würde aber bedeuten, dass jedes Verhalten im Vorhinein explizit festgelegt wurde, was bei steigender Komplexität der Aufgaben nicht möglich ist, womit auch Computerprogramme komplexer und damit weniger explizit vorprogrammiert sind. ${ }^{375}$ Die Anzahl der zu berücksichtigenden Faktoren (Variablen) kann die menschliche Auffassungsgabe schnell überschreiten, insbesondere, wenn Berechnungen für die Entscheidungsfindung - wie bei Künstlicher Intelligenz - stattfinden. Aus diesem Grund lohnt vorab eine Untersuchung der Willenserklärungen unter Beteiligung herkömmlicher technischer Einrichtungen, wobei hier schon zwei Phänomene denkbar sind: Einerseits die reine Verkörperung des Willens des Nutzers durch Erzeugung einer Erklärung mit einem genauen und bekannten Inhalt und andererseits eine vorprogrammierte Reaktion auf verschiedene - aber vorab absehbare - Zustände, bei welchen der genaue Erklärungsinhalt dem Benutzer nicht bekannt ist, beispielsweise bei BietAgenten. ${ }^{376}$

\section{Verkörperung und Übertragung mithilfe von Computern}

Bedient sich eine Person zur Verkörperung und Übermittlung einer Willenserklärung eines Computers - ohne dass weitere Regeln auf mögliche Reaktionen der Gegenseite definiert werden - so ist diese Willenserklärung einem Brief ähnlich. Dabei übernimmt der Computer bzw. die beteiligten Computer im Übertragungsweg einerseits die Funktion des Übermittlers - wie ein (elektronischer) Bote - und andererseits die Funktion des Mediums zur Verkörperung. Diese Art von (elektronischen) Willenserklärungen wird seit der vermehrten Verbreitung und Vernetzung von Computern diskutiert, ${ }^{377}$ insbesondere im Hinblick auf Vertragsschluss und Willenserklärungen im Internet. ${ }^{378}$ Allerdings ist das Phänomen nicht auf den Vertragsschluss beschränkt, beispielsweise ist auch die Ausübung von Gestaltungsrechten - wie die Anfechtung - denkbar.

374 MünchKommBGB/Säcker, Einl. BGB Rn. 189; Palandt/Ellenberger, Einf. v. $\S 116$ BGB Rn. 1; Staudinger/Singer, Vorbem. Zu §§ 116-144 BGB Rn. 57; Medicus/Petersen, Rn. 256; auch noch Wolf/Neuner ${ }^{l 1}$, § 31 Rn. 10, seit der 12. Auflage wird differenziert, vgl. Neuner, § 32 Rn. 39 ff.

375 Ebenso Janal, AcP 215 (2015), 830, 837.

376 Hoeren/Sieber/Holznagel/Kitz, Teil 13.1 Rn. $37 \mathrm{ff}$.

377 Vgl. Petersen, Jura 2002, 387 ff.; Clemens, NJW 1985, 1998; Fritzsche/Malzer, DNotZ 1995, 3 ff.; Köhler, AcP 182 (1982), 126 ff; Wiebe, S. 97 ff.

378 Vgl. Taupitz/Kritter, JuS 1999, 839 ff. 
a) Vorliegen einer Willenserklärung

Untersucht man diese Erklärungen hinsichtlich der Anforderungen an eine Willenserklärung, so ergeben sich keine weiteren Schwierigkeiten, die über diejenigen anderer Willenserklärungen hinausgehen, weder bei der inhaltlichen Bestimmtheit noch bei der Zuordnung zu einer Person. ${ }^{379}$ Der objektive Tatbestand ist problemlos erfüllt, wenn derjenige, der den Computer bedient, aus Sicht eines objektiven Dritten - beispielsweise durch eine E-Mail, einen Chat oder durch Auslösen eines Bestellvorgangs - derart einen Willen kundgibt, dass eine Rechtsfolge herbeigeführt werden soll. ${ }^{380}$ Auch beim subjektiven Tatbestand bestehen nur die bekannten Probleme, da sich auf das Gewollte des handelnden Subjekts bezogen wird. Der Handlungswille liegt vor, sobald der Benutzer bewusst eine Handlung beispielsweise über ein Eingabegerät (Tastatur, Maus, Mikrofon o.ä.) tätigt, nicht jedoch bei Reflexen oder einem ungewollten Mausklick. ${ }^{381}$ Der Erklärungswille liegt vor, wenn bei Formulierung der Erklärung und bei Vollführung der Handlung durch Bedienung oder - seltener - durch direkte Programmierung dem Erklärenden bewusst ist, dass er rechtsgeschäftlich handelt. Dies ist nicht der Fall, wenn der Erklärende davon ausgeht, nur Informationen anzufordern, ohne dass erkennbar ist, dass eine rechtsverbindliche Erklärung abgegeben wird. ${ }^{382}$ Zumindest bei einem Vertragsschluss im elektronischen Geschäftsverkehr bestehen für Unternehmer gem. $\S 312 \mathrm{j}$ Abs. 3 BGB besondere Verpflichtungen. ${ }^{383}$ Der Geschäftswillen des Benutzers beurteilt sich bei elektronischen Willenserklärungen nicht anders als bei anderen (herkömmlichen) Willenserklärungen.

\section{b) Wirksamkeit}

Neben den Voraussetzungen, die erfüllt sein müssen, damit überhaupt eine Willenserklärung vorliegt, bestehen weitere Wirksamkeitsvoraussetzungen. Die Wirksamkeit von - den im Weiteren nur relevanten - empfangsbedürftigen Willenserklärungen wird für Willenserklärungen unter

379 Vgl. BGH, U. v. 07.11.2001 - VIII ZR 13/01, NJW 2002, 363, 364 f.; Geis, NJW 1997, 3000; Fritzsche/Malzer, DNotZ 1995, 3, 8.

380 Vgl. Köhler, § 6 Rn. 2.

381 Spindler/Schuster/Spindler, Vorb. zu $\S 116$ ff. BGB Rn. 3.

382 Vgl. Spindler/Schuster/Spindler, Vorb. zu $\S 116$ ff. BGB Rn. 4.

383 Spindler/Schuster/Schirmbacher, § 312j BGB Rn. 36 ff. 
Abwesenden in $\S 130$ BGB geregelt: namentlich Abgabe und Zugang. ${ }^{384}$ Diese Wirksamkeitsvoraussetzungen müssen allerdings auch bei Willenserklärungen unter Anwesenden vorliegen. ${ }^{385}$ Bei den bisher betrachteten elektronischen Willenserklärungen bestehen zunächst keine spezifischen Probleme der Wirksamkeit. ${ }^{386}$ Allerdings werden die Fragen der Wirksamkeit einer Willenserklärung im weiteren Verlauf bei der Erörterung des technischen Phänomen der Künstlichen Intelligenz eine wichtige Rolle spielen, sodass die Grundlagen der relevanten Aspekte kurz vorab erläutert werden.

\section{aa) Abgabe}

Die Voraussetzungen, die erfüllt sein müssen, damit eine Willenserklärung abgegeben ist, sind in manchen Teilen unklar. ${ }^{387}$ Üblicherweise wird zumindest verlangt, dass der Erklärende alles Erforderliche getan haben muss, damit die Erklärung wirksam werden kann ${ }^{388}$ Fraglich ist dann aber schon, ob dies mit dem Willen des Erklärenden geschehen muss ${ }^{389}$ - also ein subjektives Element besteht - oder ob es ausreicht, dass aus objektiver Sicht alles Erforderliche getan wurde. ${ }^{390}$ Für das Vorhandensein eines Abgabewillens spricht der Umgang mit abhandengekommenen Willenserklärungen. Dies sind Willenserklärungen, die ohne den Willen des Erklärenden entäußert wurden und daher als nicht abgegeben gelten. ${ }^{391}$ Teilweise wird eine Ausnahme gemacht, wenn der Erklärende hätte erkennen

384 Flume, S. 223.

385 Vgl. BeckOGK/Gomille, § 130 BGB Rn. 1.

386 Vgl. für automatische Willenserklärungen D. Paulus, JuS 2019, 960, 963.

387 Staudinger/Singer/Benedict, § 130 Rn. 28 geht davon aus, dass »weitestgehend Einigkeit « besteht.

388 BGH, U. v. 18.12.2003 - IV ZR 39/02, NJW-RR 2003, 384; BeckOGK/Gomille, $\S 130$ BGB Rn. 36; Heidel/Hüßtege/Mansel/Noack/Faust, § 130 BGB Rn. 5; MünchKommBGB/Einsele, § 130 BGB Rn. 13; Palandt/Ellenberger, § 130 BGB Rn. 4; Grigoleit/Herresthal, Rn. 10; Medicus/Petersen, Rn. 263 ff.

389 BGH, U. v. 18.12.2002 - IV ZR 39/02, NJW-RR 2003, 384; BGH, U. v. 30.05.1975 - V ZR 206/73, NJW 1975, 2101, 2102 = BGHZ 65, 13; BeckOK/Wendtland, § 130 BGB Rn. 5; Jauernig/Mansel, § 130 BGB Rn. 1; Bork, Rn. 615; auch »Vollendung des Willens«, Staudinger/Singer/Benedict, § 130 BGB Rn. 27.

390 Moussa, S. 116.

391 BGH, U. v. 08.03.2006 - IV ZR 145/05, Rn. 29, NZA-RR 2006, 847. 
und vermeiden können, dass die Erklärung entäußert wurde. ${ }^{392}$ Daher liegt es nahe, den Abgabewillen mit dem Erklärungsbewusstsein derart gleichzusetzen, dass bei seinem Fehlen vom fehlenden Erklärungsbewusstsein ausgegangen wird. ${ }^{393}$ Allerdings setzt der Abgabewillen an einem anderen Moment an: Während die subjektiven Tatbestandsmerkmale der Willenserklärung auf die Erklärung selbst abzielen, zielt der Abgabewille auf das Wirksamwerden ab. ${ }^{394}$ Wenn schon keine Handlung vorliegt, die eine Abgabe sein kann, so fehlt auch der Handlungswille. ${ }^{395}$ Daraus folgt, dass auch bei der Abgabe ein Willensmoment besteht.

Bei empfangsbedürftigen Willenserklärungen muss noch ein weiteres Element hinzukommen. In der Regel wird verlangt, dass der Erklärende die Erklärung in Richtung des Empfängers in den Verkehr bringt, sodass ohne Weiteres der Zugang eintreten kann. ${ }^{396}$ Hier sind sodann zwei Punkte fraglich: Erstens, ob der Akt des Inverkehrbringens zur Abgabe gehört und zweitens, ob sie in Richtung des Empfängers in den Verkehr gebracht werden muss oder ob nicht allgemeines Inverkehrbringen ausreicht. Die Frage, ob der Akt des Inverkehrbringens Bestandteil der Abgabe ist, hat insbesondere im Kontext elektronischer Willenserklärungen Relevanz, da durch entsprechende Programmierung des Computers eine erhebliche Verzögerung zwischen dem Zeitpunkt, an dem alles Erforderliche getan wurde, damit die Erklärung in den Verkehr gerät und dem eigentlichen Inverkehrbringen liegt. Würde das Inverkehrbringen noch zur Abgabe gehören, so würde eine zwischenzeitlich eintretende Geschäftsunfähigkeit oder der Tod des Erklärenden die Erklärung - im Gegensatz zu einer abgegebenen Erklärung gem. $§ 130$ Abs. 2 BGB - nicht wirksam werden lassen, obwohl objektiv alles getan wurde, damit sie wirksam werden kann. Insoweit spricht einiges dafür, dass der Akt des eigentlichen Inverkehrbringens nicht mehr Bestandteil der Abgabe ist, wenn sich hierfür Hilfsmittel oder Hilfspersonen bedient wird. ${ }^{397}$

Ein Teil des Schrifttums geht zudem davon aus, dass allgemeines Inverkehrbringen ausreichend ist und daher die Erklärung nicht in Richtung

392 BeckOGK/Gomille, § 130 BGB Rn. 43; MünchKommBGB/Einsele, § 130 BGB Rn. 14; Palandt/Ellenberger, § 130 BGB Rn. 4.

393 Heidel/Hüßtege/Mansel/Noack/Faust, § 130 BGB Rn. 9; a.A. Bork, Rn. 615.

394 Ebenso wohl auch Medicus/Petersen, Rn. 267.

395 BeckOK/Wendtlandt, § 130 BGB Rn. 6.

396 BeckOK/Wendtlandt, § 130 BGB Rn. 6; MünchKommBGB/Einsele, § 130 BGB Rn. 13; Palandt/Ellenberger, § 130 BGB Rn. 4; Flume, S. 225; Köhler, § 6 Rn. 12.

397 Neuner, §33 Rn. 3; Wiebe, S. 390 sieht allerdings in der Regel ein Zusammenfallen von »Kodierung und Absenden«. 
des Empfängers in den Verkehr gebracht werden muss. ${ }^{398}$ Argumentiert wird auch damit, dass eine Erklärung, die mit Willen des Erklärenden nach außen kundgetan wurde, auch wenn sie allgemein in den Verkehr gebracht wurde, den Weg zum richtigen Empfänger finden kann. Nur dieses Risiko wäre dem Erklärenden zu Last zu legen. ${ }^{399}$ Eine Erklärung dürfte dann »in den Verkehr« gelangt sein, wenn die Erklärung in ein geeignetes Computernetzwerk gegeben wurde, sodass der Zugang beim Empfänger eintreten kann. Da hier jegliche Computernetzwerke denkbar sind und somit keine Beschränkung auf das sog. »Internet $\aleph^{400}$ besteht, sind somit Fälle möglich, bei welchen der Empfänger nicht an das entsprechende Computernetzwerk angebunden ist, sodass der Zugang nie eintreten kann. Es handelt sich dann um ein ungeeignetes Computernetzwerk. Zumindest, wenn dem Erklärenden dieser Umstand bekannt ist, scheint es bedenklich, dass in diesem Fall dennoch eine Abgabe vorliegen soll. Freilich würde die Wirksamkeit dann nach wie vor am Zugang scheitern, sodass die Frage bei elektronischen Willenserklärungen nur eine untergeordnete Rolle spielt.

Damit lässt sich festhalten, dass sich die Abgabe von Willenserklärungen in einen subjektiven und einen objektiven Bestandteil zerlegen lässt: Subjektiv muss beim Erklärenden der Wille vorhanden sein, die Erklärung endgültig kundzutun, das umfasst bei empfangsbedürftigen Willenserklärungen auch den Willen, dass die Erklärung in den Verkehr gelangt. Objektiv müssen alle Handlungen vollendet worden sein, die erforderlich sind, damit die Erklärung wirksam werden kann, wobei der Akt des Inverkehrbringens selbst nicht zur Abgabe gehört. Für eine elektronische Willenserklärung heißt das, dass die Abgabe dann vorliegt, wenn der Erklärende die Nachricht derart abspeichert, dass ohne weiteres Zutun die Erklärung durch ein geeignetes Computernetzwerk übertragen werden kann, ${ }^{401}$ wobei von dem Computernetzwerk angenommen werden muss, dass die Nachricht übermittelt wird. Auf eine Bestätigung des Sendebefehls kommt es nicht an, ${ }^{402}$ da eine entsprechende Bestätigung nur fakultativ und

398 Staudiner/Singer/Benedict, § 130 BGB Rn. 33; Neuner, § 33 Rn. 6; kritisch auch BeckOGK/Gomille, § 130 BGB Rn. $38.1 \mathrm{f}$.

399 Neuner, § 33 Rn. 6.

$400 \mathrm{Zu}$ Deutsch auch »Zwischennetz«, was vom Namen her schon darlegt, dass es sich dabei um eine Verbindung von verschiedenen Netzwerken handelt, vgl. Tanenbaum/Wetherall, S. 51 f., 80 ff. Findet in Teilnetzen eine Filterung statt, auf die der Empfänger keinen Einfluss hat - beispielsweise durch staatliche Stellen - so geht dies zu Lasten des Erklärenden.

401 Vgl. MünchKommBGB/Einsele, § 130 BGB Rn. 13; Petersen, Jura 2002, 387; Fritzsche/Malzer, DNotZ 1995, 3, 11.

402 So aber Hoeren/Sieber/Holznagel/Kitz, Teil 13.1 Rn. 58. 
damit von der Programmierung der verwendeten Software abhängig ist. $\mathrm{Ob}$ die Erklärung erst zeitversetzt oder mit Bedingungen in den Verkehr gelangt, ist nicht von Relevanz. Mit dem Abspeichern und der Programmierung des Zeitpunkts oder mit dem Versehen von Bedingungen hat der Erklärende alles Erforderliche getan, damit die Erklärung wirksam werden kann. ${ }^{403}$

\section{bb) Zugang}

Da es sich bei den elektronischen Willenserklärungen der herkömmlichen technischen Phänomene grundsätzlich um verkörperte Willenserklärungen handelt, bleibt für die Frage des Zugangs nur fraglich, ob es sich um eine Willenserklärung unter Anwesenden oder unter Abwesenden handelt. ${ }^{404} \mathrm{Ob}$ eine Willenserklärung unter Anwesenden vorliegt, kann aber bezweifelt werden. Selbst wenn die Kenntnisnahme ohne relevante Verzögerung stattfindet und eine Reaktion technisch sofort möglich ist - beispielsweise per Chat - ist nicht sichergestellt, dass die Erklärung sofort zur Kenntnis genommen wird. ${ }^{405}$ Somit handelt es sich immer um eine Willenserklärung unter Abwesenden. ${ }^{406}$ Gem. $§ 130$ Abs. 1 S. 1 BGB wird die Willenserklärung unter Abwesenden dann wirksam, wenn sie zugeht, also dann, wenn sie in den Machtbereich des Empfängers gelangt ist und üblicherweise mit einer Kenntnisnahme zu rechnen ist. ${ }^{407}$ Fraglich kann zwar sein, wann eine Erklärung im Machtbereich liegt; es kann aber davon ausgegangen werden, dass das der Fall ist, sobald die Willenserklärung in einer von Erklärungsempfänger im Rechts- und Geschäftsverkehr benutzten

403 Neuner, § 33 Rn. 3; a.A. Hoeren/Sieber/Holznagel/Kitz, Teil 13.1 Rn. 60; Cornelius, MMR 2002, 353, $355 \mathrm{f}$.

404 Fritzsche/Malzer, DNotZ 1995, 3, 9 ff.

$405 \mathrm{Im}$ Ergebnis ebenso Hoeren/Sieber/Holznagel/Kitz, Teil 13.1 Rn. 64. Das ändert sich auch nicht, wenn Lesebestätigungen zurückgesendet werden, da auch diese nicht in jedem Fall sofort stattfinden, sondern möglicherweise zeitverzögert. A.A. für sog. »Online-Verfahren«, MünchKommBGB/Einsele, § 130 BGB Rn. 18.

406 Hoeren/Sieber/Holznagel/Kitz, Teil 13.1 Rn. 80; a.A. Fritzsche/Malzer, DNotZ 1995, 3, 11; Taupitz/Kritter, JuS 1999, 839, 841.

407 BGH, B. v. 21.06.2011 - II ZB 15/10, Rn. 15, NJW-RR, 2011, 1184; BGH, U. v. 21.01.2004 - XII ZR 214/00, NJW 2004, 1320; BGH, U. v. 26.11.1997 VIII ZR 22/97, NJW 1998, 976, 977; BGH, U. v. 27.10.1982 - V ZR 24/82, NJW 1983, 929, 930; BGH, U. v. 13.02.1980 - VIII ZR 5/79, NJW 1980, 990; BGH, U.v. 03.11.1976 - VIII ZR 140/75, NJW 1977, $194=$ BGHZ 67, 271; BeckOGK/Gomille, § 130 BGB Rn. 45; MünchKommBGB/Einsele, § 130 BGB Rn. 27; Palandt/Ellenberger, $\S 130$ BGB Rn. 5. 
Empfangseinrichtung - beispielsweise in einem E-Mail-Postfach - zugegangen ist. ${ }^{408}$ Wann heutzutage üblicherweise mit einer Kenntnisnahme zu rechnen ist, ist im Kontext elektronischer Willenserklärung nicht anders zu beurteilen als bei gewöhnlichen verkörperten Willenserklärungen unter Abwesenden. ${ }^{409}$

\section{Zuordnungsprobleme bei der Beteiligung von Dritten}

Möglich ist auch, dass Dritte an der Erklärung beteiligt sind, die mit einer herkömmlichen technischen Einrichtung verkörpert oder übertragen wird. Grundsätzlich sind hier die Regelungen der Stellvertretung der $\S \S 164 \mathrm{ff}$. BGB anwendbar. ${ }^{410}$ Da aber das Handeln durch den Dritten für den Erklärungsempfänger in gewissen Konstellationen nicht ersichtlich ist, können in diesen Fällen besondere Zuordnungsprobleme entstehen; insbesondere, wenn der Dritte ohne Vertretungsmacht handelt. Seitens der Rechtsprechung wird in diesem Kontext die Möglichkeit einer Duldungsoder Anscheinsvollmacht diskutiert. ${ }^{411}$ Eine Duldungsvollmacht liegt dann vor, wenn der Vertretene den handelnden Dritten wissentlich als seinen Vertreter duldet und der Erklärungsempfänger diese Duldung nach Treu und Glauben als Vollmacht auffassen kann. ${ }^{412}$ Üblicherweise ist das der Fall, wenn der handelnde Dritte wiederholt als Vertreter auftritt oder der Vertretene die Möglichkeit der Handlung unterbinden kann. ${ }^{413}$ Eine Anscheinsvollmacht wird dagegen dann angenommen, wenn der Erklärungsempfänger auf den Bestand einer Vollmacht des handelnden

408 MünchKommBGB/Einsele, § 130 BGB Rn. 18; Taupitz/Kritter, JuS 1999, 839, 841 f.; vgl. hierzu ausführlich Greiner/Kalle, JZ 2018, 535 ff.

409 Vgl. zum Streitstand BeckOGK/Gomille, § 130 BGB Rn. 62 ff., 74 ff.

410 Hoeren/Sieber/Holznagel/Kitz, Teil 13.1 Rn. $125 \mathrm{ff}$.

411 BGH, U. v. 11.05.2011 - VIII ZR 289/09, NJW 2011, 2421, 2422 f. = BGHZ 189, 346; vgl. auch BGH, U. v. 26.01.2016 - XI ZR 91/14, Rn. 56 ff., NJW 2016, 2024 $=$ BGHZ 208, 331 .

412 BAG, U. v. 23.02.2017 - 6 AZR 665/15, Rn. 45, NJW 2017, 2698.

413 BGH, U. v. 22.07.2014 - VIII ZR 313/13, Rn. 26, NJW 2014, $3150=$ BGHZ 202, 158; BGH, U. v. 10.01.2007 - VIII ZR 380/04, Rn. 19, NJW 2007, 987; BGH, U. v. 21.06.2005 - XI ZR 88/04, NJW 2005, 2985, 2987; BGH, U. v. 20.04.2004 -XI ZR 164/03, NJW 2004, 2745, 2747 f.; BGH, U. v. 14.05.2002 - XI ZR 155/01, NJW 2002, 2325, 2327; MünchKommBGB/Schubert, § 167 BGB Rn. 106; Neuner, $\S 50$ Rn. 86 f. 
Dritten vertraut und der Vertretene trotz Unkenntnis der Erklärungshandlung diese hätte erkennen und vermeiden können. ${ }^{414}$

Bei den Rechtsscheintatbeständen der Duldungs- oder Anscheinsvollmacht vertraut der Erklärungsempfänger aber auf den Bestand der Vollmacht, da ihm bekannt ist, dass ein Dritter für den Vertretenen handelt. Deshalb kritisiert die Literatur diesen Ansatz bei elektronischen Willenserklärungen zu Recht. Hier verwendet der handelnde Dritte in der Regel ein Legitimationszeichen des Vertretenen, ${ }^{415}$ sodass der Erklärungsempfänger von einer Erklärung des Vertretenen und nicht vom eigentlich erklärenden Dritten ausgeht; der handelnde Dritte offenbart sich ihm in der Regel nicht. Der Erklärungsempfänger vertraut daher nicht auf die - ihm sich sonst offenbarende - Vollmacht, sondern auf den Rechtsschein der Erklärung. Es macht auch keinen Unterschied, ob eine erste Verwendung oder eine wiederholende Verwendung vorliegt. Aus diesen Gründen ist sowohl die Duldungs- als auch die Anscheinsvollmacht bei einer missbräuchlichen Verwendung von Legitimationszeichen ungeeignet. ${ }^{416}$

Näher liegt in diesen Fällen der Vergleich mit der Konstellation des $\S 172$ Abs. 1 BGB. Hier vertraut der Erklärungsempfänger in eine Vollmachtsurkunde, die der Vertreter vorlegt. Auch wenn die Norm - mangels Vollmachtsurkunde in Schriftform gem. §126 BGB - nicht direkt anwendbar ist, ist eine analoge Anwendung denkbar. ${ }^{417}$ Hierfür müssen drei Voraussetzungen gegeben sein: Erstens ein objektiver Rechtsschein, der bei Handeln im fremden Namen an der Art und der Möglichkeit des Zugangs des Legitimationszeichens zu beurteilen ist, zweitens die Zuordnung zum Vertretenen bzw. Inhaber des Legitimationskennzeichens und drittens muss der Erklärungsempfänger gutgläubig sein. ${ }^{418} \mathrm{Im}$ Rahmen der Prüfung des

414 »Eine Anscheinsvollmacht setzt voraus, dass der Vertretene das Handeln des Scheinvertreters nicht kennt, er es aber bei pflichtgemäßer Sorgfalt hätte erkennen und verhindern können und der Geschäftspartner annehmen durfte, der Vertretene kenne und billige das Handeln des Vertreters«, BGH, U. v. 26.01.2016 - XI ZR 91/14, Rn. 61, NJW 2016, 2024 = BGHZ 208, 331 m.w.N. Grundsätzlich wird die Anscheinsvollmacht von Teilen der Literatur kritisch gesehen, vgl. Neuner, § 50 Rn. 94 ff.

415 Beispielsweise eine Kombination aus Nutzername und Passwort oder ein Zertifikat.

416 MünchKommBGB/Schubert, § 167 BGB Rn. 128; Staudinger/Schilken, § 167 BGB Rn. 35; Herresthal, in: Taeger/Wiebe, S. 27 ff.; Faust, JuS 2011, 1027, 1028; Herresthal, JZ 2017, 28, 32 f.; Herresthal, JZ 2011, 1171, 1172 ff.; Sonnentag, WM 2012, 1614, 1615.

417 Staudinger/Schilken, § 172 BGB Rn. 8; Borges, NJW 2011, 2400, 2403 f.; Oechsler, MMR 2011, $631 \mathrm{f}$.

418 Vgl. ausführlich Herresthal, in: Taeger/Wiebe, S. 32 ff. 
objektiven Rechtsscheins macht es einen Unterschied, ob das Legitimationszeichen bewusst überlassen wurde oder der Dritte anderweitig davon Kenntnis erlangt hat. Im ersten Fall liegt ein Aushändigen des Legitimationszeichens vor, sodass vorsätzlich ein Rechtsschein geschaffen wurde und damit die Voraussetzungen der Anwendung des $\S 172$ Abs. 1 BGB vorliegen. Damit haftet der Inhaber des Legitimationszeichens auf das positive Interesse. ${ }^{419}$ Anders ist dies im zweiten Fall, da dort das Legitimationszeichen nur fahrlässig abhandengekommen ist und somit die analoge Anwendung des $\S 172$ Abs. 1 BGB ausscheidet. ${ }^{420}$ Denkbar ist aber eine Haftung aus culpa in contrahendo gem. $\S \S 280$ Abs. 1 S. 1, 311 Abs. 2 Nr. 3, 241 Abs. 2 BGB. ${ }^{421}$ Denn auch das fahrlässige Setzen eines Anscheines ist ein ähnlicher geschäftlicher Kontakt i.S.d. § 311 Abs. 2 Nr. 3 $\mathrm{BGB},{ }^{422}$ sodass zwischen dem Inhaber des Legitimationszeichens und dem Erklärungsempfänger ein vorvertragliches Vertrauensverhältnis begründet werden kann. Die Haftung ist dann aber auf das negative Interesse beschränkt. ${ }^{423} \mathrm{Zu}$ prüfen ist indessen, ob ein Vertretenmüssen des Inhabers des Legitimationszeichens für die missbräuchliche Nutzung vorliegt, genauer, ob er den Missbrauch des Legitimationszeichens hätte erkennen und vermeiden können. Hier ist sodann auf die Sicherheitsstandards abzustellen, wie das Legitimationszeichen üblicherweise gegen unbefugten Zugriff geschützt wird. ${ }^{424}$

\section{Komplexe aus (vorprogrammierten) Willenserklärungen}

Ein weiterer zu betrachtender Faktor bei vorprogrammierten, elektronisch verkörperten und übermittelten Willenserklärungen ist, das durch den Benutzer nicht mehr eine einzelne Willenserklärung für einen bestimmten Fall erzeugt wird, sondern mehrere Willenserklärungen - als ein Komplex von Willenserklärungen - erzeugt werden können. Klassische Anwendungsbereiche finden sich im Internethandel (teilweise »E-Commerce« genannt ${ }^{425}$ ), insbesondere in Online-Shops. Üblicherweise wird bei den dort

419 Herresthal, JZ 2011, 1171, 1174.

420 Oechsler, MMR 2011, 631, 632 f.; Sonnentag, WM 2012, 1614, 1618.

421 Medicus/Petersen, Rn. 971; Sonnentag, WM 2012, 1614, 1619; Oechsler, MMR 2011, 631, 633; ablehnend noch Herresthal, in: Taeger/Wiebe, S. 40 f.

422 BeckOGK/Herresthal, § 311 BGB Rn. 293.

423 MünchKommBGB/Schuster, § 311 BGB Rn. 26.

424 Herresthal, in: Taeger/Wiebe, S. 37 ff.; Oechsler, MMR 2011, 631, 633; vgl. auch LG Bonn, U. v. 19.12.2003 - 2 O 472/03, MMR 2004, 179.

425 Vgl. Tamm/Tonner/Brönneke/Brönnecke, § 10 Rn. 1. 
angezeigten Waren und Dienstleistungen davon ausgegangen, dass kein rechtsverbindliches Angebot, sondern eine invitatio ad offerendum vorliegt. ${ }^{426}$ Dies ist allerdings im Rahmen der Auslegung zu ermitteln. ${ }^{427}$ Insoweit fallen die Willenserklärungen, die Angebote sind und nur noch durch einen unbestimmten Empfängerkreis angenommen werden müssen, nicht in diese Kategorie; ${ }^{428}$ hier handelt sich um den Fall der offerta ad incertas personas $;{ }^{429}$ es besteht bei diesen Willenserklärungen kein Unterschied zum klassischen Warenautomaten. ${ }^{430}$ Anders ist die Situation aber zu beurteilen, wenn der Computer nach vordefinierten Regeln - auf Vorrat - vorprogrammierte Willenserklärungen abgeben und übertragen soll, insbesondere beim Vertragsschluss. Diesen Willenserklärungen ist aber gemein, dass sie nach wie vor der Mensch erklärt. ${ }^{431}$ Solche Erklärungen werden teilweise als automatisierte Willenserklärungen bezeichnet. ${ }^{432}$

\section{a) Automatische Annahmen}

Ein einfaches Beispiel für solche Komplexe von Willenserklärungen sind automatische Annahmen (Auto-Reply), ${ }^{433}$ bei welchen eingehende Bestellungen - als Angebot - automatisiert, aber vorbehaltlos akzeptiert werden. Zwar kann man auch die spätere Leistungserbringung als konkludente Annahme sehen, allerdings würde der Vertragsschluss zeitlich dann möglicherweise später - wenn nicht gar nach der Annahmefrist i.S.d. § 147 Abs. 2 BGB - liegen, sodass es auf den Zeitpunkt der Annahme ankommt. Damit eine Annahme erklärt werden kann, muss ein entsprechendes Angebot vorliegen. An dessen Wirksamkeit bestehen keine Zweifel, da

426 BGH, U. v. 16.10.2012 - X ZR 37/12, Rn. 14, NJW 2013, 598 = BGHZ 195, 126; BGH, U. v. 03.05.2012 - III ZR 62/11, Rn. 11, NJW 2012, 2268; BGH, U. v. 26.01.2005 - VIII ZR 79/04, NJW 2005, 976; Grigoleit, NJW 2002, 1151, 1158.

427 MünchKommBGB/Busche, § 145 BGB Rn. 13 ff.; Mehrings, MMR 1998, 30, 32; $\mathrm{zu}$ denken ist hier beispielsweise an einen Internetshop mit zum Download angebotenen Waren, vgl. Köhler, § 8 Rn. 59.

428 Was durch Auslegung zu ermitteln ist, vgl. BGH, U. v. 03.05.2012 - III ZR 62/11, Rn. 11, NJW 2012, 2268.

429 Vgl. Köhler, § 8 Rn. 59; a.A. MünchKommBGB/Säcker, Einl. BGB Rn. 191.

430 Vgl. Fritzsche, JA 2006, 674, 675.

431 Vgl. BGH, U. v. 16.10.2012 - X ZR 37/12, Rn. 17, NJW 2013, 598 = BGHZ 195, 126.

432 Spindler/Schuster/Spindler, Vorb. zu $§ § 116$ ff. BGB Rn. 5; Boemke/Ulrici, $\S 5$ Rn. 28.

433 Vgl. MünchKommBGB/Busche, § 147 BGB Rn. 4. 
auch das automatisch - wenn auch programmiert - erklärende Computersystem eine geeignete Empfangseinrichtung für verkörperte Willenserklärungen unter Abwesenden darstellt und es daher nur noch auf die mögliche Kenntnisnahme des Benutzers ankommt.

Da die Annahme eine Willenserklärung ist, ist fraglich, ob entsprechende automatisiert generierte Antworten überhaupt eine Willenserklärung sind. Insbesondere bei Verbraucherverträgen besteht im elektronischen Geschäftsverkehr gem. §312i Abs. 1 Nr. 3 BGB die Pflicht, den Eingang einer Bestellung unverzüglich zu bestätigen, was in der Regel automatisiert geschieht. ${ }^{434}$ Abgrenzungsprobleme können hier zwischen einer automatisch generierten Bestellbestätigung - als Wissenserklärung ${ }^{435}$ - und einer Annahmeerklärung bestehen. ${ }^{436}$ Bei Formulierungen, die eine baldige oder schnelle Ausführung der Bestellung ankündigen, wurde seitens der Rechtsprechung eine automatisierte Annahmeerklärung angenommen. ${ }^{437}$ Dabei ist darauf abzustellen, ob die Äußerungen in der automatisch generierten Antwort unter dem objektiven Empfängerhorizont als vorbehaltloses Akzeptieren des Angebots und damit gem. $\S \S 133,157$ BGB als Annahme zu werten sind. ${ }^{438}$ Die notwendigen subjektiven Voraussetzungen an eine Willenserklärung werden mit der Inbetriebnahme und Programmierung des Computersystems, also der Formulierung der Erklärung und der Einstellung der Reaktion auf eingehende Nachrichten, erfüllt. Zwar besteht möglicherweise kein Erklärungsbewusstsein des Benutzers, da ihm nicht bewusst ist, dass seine vorformulierte Erklärung eine rechtliche Folge hat, allerdings könnte der Erklärungsempfänger die Erklärung als Annahme auffassen und der Benutzer bzw. Verwender hätte mit der im Verkehr üblichen Sorgfalt erkennen und vermeiden können, dass er eine entsprechende

434 Vgl. MünchKommBGB/Wendehorst, § 312i BGB Rn. 97.

435 BGH, U. v. 16.10.2012 - X ZR 37/12, Rn. 19, NJW 2013, 598 = BGHZ 195, 126; OLG Düsseldorf, U. v. 19.05.2016 - I-16 U 72/15, Ls. 1, MMR 2016, 593.

436 Vgl. MünchKommBGB/Busche, § 147 BGB Rn. 4; Bodenstedt, MMR 2004, 719 ff.; Stockmar/Wittwer, CR 2005, 118, 125.

437 BGH, U. v. 16.10.2012 - X ZR 37/12, Rn. 17, NJW 2013, $598=$ BGHZ 195, 126; OLG Düsseldorf, U. v. 19.05.2016 - I-16 U 72/15, Rn. 66 f., MMR 2016, 593; OLG Düsseldorf, U. v. 20.1.2002 - 9 U 94/02, MMR 2003, 405, 406; LG Köln, U. v. 16.04.2003 - 9 S 289/02, MMR 2003, 481.

438 BGH, U. v. 26.01.2005 - VIII ZR 79/04, NJW 2005, 976; Spindler/Schuster/Spindler, $\quad$ Vorb. $\quad \mathrm{zu} \quad \S \S 116 \quad$ ff. $\quad$ BGB $\quad$ Rn. 8; Hoeren/Sieber/Holznagel/Kitz, Teil 13.1 Rn. 170; Bodenstedt, MMR 2004, 719, 720; Stockmar/Wittwer, CR 2005, 118, 125. 
Erklärung abgibt, sodass zumindest Erklärungsfahrlässigkeit ${ }^{439}$ anzunehmen ist. ${ }^{440} \mathrm{Ob}$ der nicht konstitutive Geschäftswille vorhanden ist, ${ }^{441}$ spielt für die Vorlage der Annahme keine Rolle.

Als empfangsbedürftige Willenserklärung bedarf die automatische Annahme noch der Abgabe und des Zugangs für ihre Wirksamkeit. ${ }^{442}$ Bei den erzeugten Erklärungen liegt aber für alle Erklärungen ein Abgabewillen vor. Der Erklärende hat mit der Programmierung des Computersystems auch für alle Willenserklärungen das Erforderliche getan, damit sie (potenziell) in den Verkehr gelangen und damit wirksam werden können, womit die Erklärungen auch abgegeben sind. Beim Zugang bestehen sodann keine Besonderheiten. ${ }^{443}$

\section{b) Regelbasierte Willenserklärungen}

Eine weitere Möglichkeit eines Komplexes von Willenserklärungen liegt vor, wenn durch den Computer nach vordefinierten Wenn-Dann-Regeln weitere Willenserklärungen erzeugt werden, die sich nach den (erwarteten) Handlungen der Gegenseite richten, wie beispielsweise bei Biet-Agenten. Auch hier ist die notwendige Willensbildung durch einen Menschen im Vorhinein bereits vollständig abgeschlossen. Im Gegensatz zur automatisierten Annahme bestehen auch keine Probleme beim Erklärungsbewusstsein, da hier eine zielgerichtete Verwendung entsprechender Software stattfindet. Auch hinsichtlich der Wirksamkeit unterscheidet sich die automatische und regelbasierte Willensklärung nicht von herkömmlichen elektronischen Willenserklärungen. Einziger Unterschied ist der Umstand, dass möglicherweise nicht alle Willenserklärungen in den Verkehr gelangen, was an der Wirksamkeit, der in den Verkehr gelangten und zugegangenen Willenserklärungen jedoch nichts ändert.

Allerdings zeigt das letzte Beispiel möglicherweise einen Wertungswiderspruch auf. Grundsätzlich ließe sich dieses Vorgehen auch mit einem (menschlichen) Boten umsetzen: Bedient sich der Mensch eines Boten zur Übermittlung seiner Willenserklärung, so ist denkbar, dass der Bote

439 S. o., Dichotomie von Wille und Erklärung (S. 83).

440 So wohl auch Heidel/Hüßtege/Mansel/Noack/Schulze, § 147 BGB Rn. 4.

441 Möglicherweise besteht der Wille, mit dem konkreten Anbietenden nicht zu kontrahieren.

442 Sofern kein Fall von $§ 151$ S. 1 BGB vorliegt, was hier in der Regel aber nicht der Fall ist.

443 S. o., Zugang (S. 93). 
mehrere vorab erzeugte Willenserklärungen nach einem Wenn-DannSchema übermittelt. In einem simplen Beispiel kann das erste zu übermittelnde Angebot einen Kaufpreis von 20 Euro beinhalten, bei Ablehnung durch die Gegenseite soll ein Angebot mit 21 Euro abgegeben werden, dann 22 Euro etc. bis zu einer Höchstgrenze von 25 Euro, ohne dass der Bote Entscheidungsspielraum hat und somit nach wie vor fremde Willenserklärungen übermittelt. Für die Gegenseite kann es aber so aussehen, als würde verhandelt werden, also Entscheidungsspielraum vorliegen würde. Da die Abgrenzung des Boten zum Stellvertreter aufgrund des Erscheinens der Person aus Sicht der Gegenseite ohne Rücksicht auf das Innenverhältnis zum Auftraggeber zu beurteilen ist, ${ }^{444}$ würde der eigentliche Bote de jure als Stellvertreter behandelt werden. Daran ändert sich auch dann nichts, wenn die Übertragung elektronisch erfolgt. Wenn nun aber einem Computerprogramm die gleichen, vordefinierten Regeln vorgegeben werden, ${ }^{445}$ bedient sich der Erklärende nicht mehr eines (menschlichen) Boten, sondern einer Maschine, ohne dass die Regelungen der Stellvertretung zur Anwendung kommen. Sowohl für die Gegenseite als auch für einen objektiven Dritten ist in diesem Fall aber nicht ersichtlich, dass vorgefasste Willenserklärungen übermittelt werden. Es hat den Anschein, als würde der Computer verhandeln. Bereits an diesem Punkt zeigt sich das Problem von Handlungen eines Computerprogramms nach außen. Lässt man ein Computerprogramm exakt dieselbe Tätigkeit wie durch einen Menschen ausgeübt, durchführen, entsteht dennoch ein anderes Ergebnis. Freilich bestehen bei dieser Konstellation noch keine Probleme der Willenszuweisung; das Computerprogramm handelt - sofern kein Fehler vorliegt nicht nur streng deterministisch, sondern auch gemäß dem vorprogrammierten und zurechenbaren Willen des Benutzers. ${ }^{446}$

\section{Künstliche Intelligenz als Hilfsmittel}

Bedient sich der Mensch nun einer technischen Einrichtung, die eine Künstliche Intelligenz enthält, um Willenserklärungen zu verkörpern, zu übertragen oder zu empfangen, dann ist diese Künstliche Intelligenz zunächst nur ein Hilfsmittel und damit stellt sich die Frage, ob hier Unterschiede zu

444 BAG, U. v. 13.12.2007 - 6 AZR 145/07, Rn. 15, NJW 2008, 1243; BGH, U. v. 17.02.1954 - II ZR 63/53, NJW 1954, $797=$ BGHZ 12, 327; MünchKommBGB/Schubert, § 164 BGB Rn. 72; Neuner, § 49 Rn. 16.

445 Die sehr umfangreich sein können.

446 Ebenso, Janal, AcP 215 (2015), 830, 834. 
den herkömmlichen technischen Einrichtungen bestehen. Das Mehr gegenüber den herkömmlichen technischen Einrichtungen ist eine zwar deterministisch handelnde, aber komplexe Künstliche Intelligenz. Sie soll entweder aus der Handlung des Nutzers bezüglich einer Willenskundgabe eine Erklärung erzeugen und möglicherweise auch übermitteln oder bei Erklärungen der Gegenseite eine Aktion ausführen. Prägendes Beispiel für eine solche Art der Künstlichen Intelligenz sind spracherkennende Systeme bzw. persönliche Assistenten (wie Alexa oder Siri). In der Regel handelt es um künstliche neuronale Netze, welche die Sprache als Eingabe und definierte Handlungen als Ausgabe verwenden. ${ }^{447}$ Da sie mit großen Sprachmengen angelernt wurden und auch weiter kontinuierlich angelernt werden, ${ }^{448}$ sind sie nicht mehr vorprogrammiert. Solche Systeme werden nicht nur für Bestellungen, sondern auch für andere Zwecke verwendet, beispielsweise für die Steuerung von Licht oder für Home-Entertainment-Systeme. Fraglich bleibt, ob hier Maschinen schon autonom agieren und es sich um autonome, elektronische Willenserklärungen handelt. ${ }^{449}$ Möglicherweise entscheidet die Künstliche Intelligenz - wenn auch abhängig von der komplexen Eingabe - zwischen mehreren Alternativen, beispielsweise weil die Willenskundgabe des Nutzers mehrdeutig war oder fehlerhaft verstanden wurde. Somit würde möglicherweise nicht mehr ein (definierter) Wille des Benutzers kundgetan, sondern es bestünden mannigfaltige Möglichkeiten der Willenskundgabe, die auch über den eigentlichen Willen des Benutzers hinausgehen können. Aus diesem Grund wird gefolgert, dass für diese Art von Künstlicher Intelligenz keine Analogie zum Boten mehr möglich sei. ${ }^{450}$

Sofern sich der Mensch einer solchen (einfachen) Künstlichen Intelligenz als Hilfsmittel bedient und die Künstliche Intelligenz auf das zielgerichtete Verhalten des Benutzers hin die korrekte Erklärung erzeugt, bestehen keine Unterschiede zu herkömmlichen Computersystemen und somit keine besonderen juristischen Probleme. Es wird der kundgetane Wille des Benutzers nur verkörpert und übermittelt, gleich einem typischen E-Mail-Programm oder anderen Programmen, die herkömmlicherweise zur Verkörperung und Übermittlung von (elektronischen) Willenserklärungen verwendet werden. Der Unterschied liegt nur darin, dass nicht Tastatur und

447 Vgl. Siddhant/Goyal/Metallinou, S. 1 ff.

448 Vgl. Lowe/Pow/Serban/Pineau, in: SIGDIAL, S. 285 ff.

449 Vgl. D. Paulus, JuS 2019, 950, 965.

450 Grapentin, S. 89; Cornelius, MMR 2002, 353, 354 f.; Specht/Herold, MMR 2018, 40, 43. 
Maus als Eingabegerät verwendet werden, sondern ein Mikrofon in Verbindung mit der spracherkennenden Künstlichen Intelligenz, die damit nur Bestandteil des Eingabesystems ist. Aus diesem Grund spielt die Künstliche Intelligenz bei den zugehenden Willenserklärungen hier noch keine Rolle. Gehen dem System (verkörperte) Willenserklärungen von außen zu, so stellt das System eine Empfangseinrichtung dar, sodass der Zugang - als Willenserklärungen unter Abwesenden ${ }^{451}$ - spätestens mit dem Abspeichern der Erklärung auf dem System eintritt. Die Künstliche Intelligenz ist aber - als Eingabesystem - bei solchen Systemen an diesen Erklärungen nicht beteiligt. Entsprechende Erklärungen werden dem Benutzer in der Regel anderweitig visualisiert.

Wird eine Erklärung als Reaktion auf eine Handlung des Benutzers erzeugt, die der Benutzer so nicht abgeben wollte, so ist - unabhängig davon, ob die Künstliche Intelligenz einen (bekannten) Fehler hat oder unvorhergesehen handelt - zwischen zwei Fällen zu unterscheiden: Wollte der Benutzer eine andere rechtsverbindliche Erklärung abgeben, so fehlt ihm lediglich der nicht konstitutive Geschäftswille; ${ }^{452}$ die konstitutiven objektiven und subjektiven Voraussetzungen der Willenserklärung sowie die Abgabe liegen vor. Problematisch kann es werden, wenn die Künstliche Intelligenz eine Erklärung abgibt, ohne dass der Benutzer dies wollte. Erkennt eine Künstliche Intelligenz beispielsweise einen Sprachbefehl, der eigentlich eine Steuerung des Lichts bezweckte, als Bestellung, sieht die erzeugte Erklärung für einen objektiven Dritten so aus, als wollte sich der Benutzer rechtlich binden; ergo ist der objektive Tatbestand einer Willenserklärung erfüllt. Auch Handlungswille liegt vor, da immerhin noch eine Handlung gegenüber der Künstlichen Intelligenz - hier das Sprechen vorliegt. Das Erklärungsbewusstsein kann aber schon nicht mehr vorliegen, da beim Steuern des Lichts kein Bewusstsein des Benutzers vorliegt, eine rechtsverbindliche Erklärung kundzutun. Sofern das System nicht nach einer Bestätigung fragt, kann der Benutzer auch nicht erkennen, dass er eine rechtsverbindliche Handlung vollzieht. Folglich liegen in diesen Fällen

451 Auch wenn das System die Erklärung vorliest und auch auf evtl. Sprachantworten reagieren kann, findet keine direkte Kommunikation mit der Gegenseite statt, sodass keine Willenserklärung unter Anwesenden vorliegt. Jede Erklärung wird verkörpert und versandt, wobei eine sofortige Übertragung nicht sichergestellt ist. Zudem ist auch nicht sichergestellt, dass die Gegenseite anwesend ist, sodass die Kenntnisnahme verzögert stattfinden kann.

452 Vgl. MünchKommBGB/Armbrüster, Vor $§ 116$ BGB Rn. 28. 
nach der klassischen Willenserklärungsdogmatik ${ }^{453}$ bereits keine Willenserklärungen vor. ${ }^{454}$

Bestehen Konstellationen mit Beteiligung Dritter, sind bei einer auf Spracheingaben reagierenden Künstlichen Intelligenz weitere Problemfälle denkbar: Wenn die Künstliche Intelligenz mit dem Account des Besitzers des Endgeräts (Benutzer) verbunden ist und ein Dritter über einen Sprachbefehl eine Willenserklärung verkörpert und überträgt - beispielsweise, indem er eine Bestellung tätigt - so bestehen zwar keine Probleme hinsichtlich der Frage, ob eine Willenserklärung vorliegt oder wirksam ist, die Willenserklärung wirkt aber für und gegen den eigentlichen Benutzer der Künstlichen Intelligenz, sodass ein Fall der Stellvertretung gem. § 164 Abs. 1 BGB anzunehmen ist. Hatte der Dritte keine Vertretungsmacht, so handelt er als Vertreter ohne Vertretungsmacht (§ 177 Abs. 1 BGB). Dieser Fall scheint ähnlich der Konstellation bei Bestellungen im Internet mit fremdem Account durch Dritte, da der Account des Benutzers bei Bestellungen als sein Legitimationszeichen mitgesendet wird. ${ }^{455}$ Es macht aber einen Unterschied, ob die Künstliche Intelligenz die Stimme des eigentlich berechtigten Besitzers erkennen konnte oder auf jede Stimme reagiert. Sollte die Künstliche Intelligenz eigentlich nur auf die Sprache des Besitzers reagieren, so ist zumindest davon auszugehen, dass dem Dritten weder vorsätzlich noch fahrlässig die Möglichkeit gegeben wurde, für den Besitzer zu handeln, sodass der Rechtsschein dem Benutzer schon nicht mehr zuzurechnen wäre. ${ }^{456}$ Ein geeigneter Sicherheitsstandard liegt damit vor. Insoweit sind diese Fälle über die Rechtsscheinhaftung lösbar.

Anders könnte es sein, wenn die Künstliche Intelligenz nicht zwischen der Stimme des Benutzers und eines Dritten unterscheidet. Dann kann die Künstliche Intelligenz jede sprachliche Erklärung als eine solche auffassen. Zunächst ist eine Analogie zum Telefonanschluss denkbar. Bei diesem soll kein Rechtsschein durch das reine Vorhalten vorliegen, da »[d]er jeweilige Nutzer dem Anbieter gegenüber anonym [bleibt]. Diesem ist nicht bekannt, ob der Anschlussinhaber selbst, eine Person, der er den Zugang zum Telefon gestattet hat, oder ein unbefugter Dritter die angebotene Verbindungsdienstleistung in Anspruch nimmt « ${ }^{457}$ Eine ähnliche Problematik stellt sich auch beim Vorhalten spracherkennender Künstlicher Intelligenz.

453 S. o., Dichotomie von Wille und Erklärung (S. 83).

454 Ähnlich Specht/Herold, MMR 2018, 40, $42 \mathrm{f}$.

455 S. o., Zuordnungsprobleme bei der Beteiligung von Dritten (S. 94).

456 Vgl. MünchKommBGB/Schubert, § 167 BGB Rn. 132.

457 BGH, U. v. 16.03.2006 - III ZR 152/05, Rn. 18, NJW 2006, 1971 = BGHZ 166, 369. 
Allerdings werden neben dem reinen Vorhalten auch Zugangsdaten - die von Dritten indes nicht eingesehen werden können - als Legitimationszeichen bewusst eingespeichert und an den Erklärungsempfänger übertragen. Zudem können diese ohne weitere technische Schutzmaßnahmen sprich ohne weitere Legitimation - benutzt werden. Das spricht zumindest für Fahrlässigkeit und damit für die bereits für vorprogrammierte elektronische Willenserklärungen erörterte Haftung aus c.i.c. Sollte diese Problematik dem Benutzer sogar bekannt sein, so dürfte dies über die Fahrlässigkeit hinaus schon Eventualvorsatz begründen. Anders ist es aber, wenn der Dritte einen für ihn nicht als rechtserheblich erkennbaren Befehl gegeben hat - beispielsweise auch einen Steuerbefehl für das Licht - der aber nach außen wie eine Willenserklärung in Erscheinung tritt. Hier kann dann erst recht nicht von einer Willenserklärung ausgegangen werden.

IV. Künstliche Intelligenz als autonomer »Erklärender«

An ihre Grenzen gelangt die bisherige Willenserklärungsdogmatik, wenn eine an sich willenlose Künstliche Intelligenz autonom Erklärungen erzeugt, die aus objektiver Sicht Willenserklärungen sind; sie also nach außen als »Erklärender« auftritt. Dies ist der Fall bei der Art der Künstlichen Intelligenz, die auf multiple Ereignisse und Zustände ihrer Umwelt losgelöst von Handlungen des Benutzers reagiert. Erst diese Art von Künstlicher Intelligenz stellt echte und vollständige autonome Systeme dar. ${ }^{458}$ Es wird nicht mehr der Wille eines Menschen kundgetan und mithilfe der Maschine erklärt, sondern das System mit der Künstlichen Intelligenz entscheidet aufgrund seiner Eingabe aus Umweltzuständen - nach stochastischen Berechnungen oder gelernten Regeln - welche Reaktion erfolgen soll und schließt somit auf einen (mutmaßlichen) Willen, ohne dass eine weitere Interaktion mit dem Benutzer stattfindet. Aus diesem Grund ist bereits das subjektive Tatbestandsmerkmal des Handlungswillens des Benutzers bei Erklärungserzeugung nicht erfüllt. Dennoch ist die Verwendung solcher Systeme beim Vertragsschluss sowohl auf der Angebots- als auch auf der Annahmeseite vorstellbar.

458 S. o., Künstliche Intelligenz und auf sie bezogene Entitäten als Rechtsobjekt(e) (S. 73). 


\section{Angebote durch autonome Systeme}

Als Beispiel für autonome Systeme auf der Angebotsseite ist insbesondere an automatisierte Bestellsysteme zu denken, ${ }^{459}$ die aufgrund des Konsumverhaltens des Benutzers versuchen, vorherzusagen, was dieser als Nächstes brauchen wird und diese Produkte autonom bestellen. Geht man davon aus, dass das Anbieten entsprechender Waren im Internet nur eine invitatio ad offerendum ist, ${ }^{460}$ erzeugen diese autonomen Systeme entsprechende Angebote. Das Verhalten des autonomen Systems erfüllt zunächst die objektiven Voraussetzungen einer Willenserklärung. Für einen objektiven Dritten sieht es so aus, als würde sich der Benutzer - dessen Legitimationszeichen mit übertragen wird - rechtlich binden wollen, sodass eine Erklärung vorliegt. Auch an der Bestimmtheit der Erklärung bestehen keine Zweifel, sie kann vom Erklärungsempfänger als Angebot aufgefasst werden; ${ }^{461}$ die essentialia negotii sind enthalten. ${ }^{462}$ Jegliche subjektiven Voraussetzungen sind aber mangels menschlicher Beteiligung an der Erklärungserzeugung und damit mangels notwendigen Willens nicht erfüllt. Hinzu kommt, dass die Abgabe der Erklärung und damit deren Wirksamkeit nicht gegeben ist. Daher erübrigt sich auch die Frage, ob die Erklärung erst mit Vervollständigung durch das autonome System abgegeben ist. ${ }^{463}$ Mangels Wissen des Benutzers über die Erklärung kann kein Wille bestehen, eine Erklärung in den Verkehr zu bringen. Daraus folgt, dass auch fraglich ist, ob überhaupt irgendwelche Handlungen durch den Benutzer vollendet werden können, damit die Erklärung in den Verkehr gebracht werden kann oder ob die Abgabe auch durch reine Untätigkeit des Benutzers geschehen kann.

Somit zeigt sich, dass es im Gegensatz zur Verwendung von Künstlicher Intelligenz als Hilfsmittel, außer der reinen Inbetriebnahme des autonomen Systems durch den Benutzer, keine weitere Handlung gibt, mit welcher

459 Vgl. Specht/Herold, MMR 2018, 40, 41.

460 S. o., Komplexe aus (vorprogrammierten) Willenserklärungen (S. 96).

461 Vgl. BGH, U. v. 16.03.2006 - III ZR 152/05, Rn. 11 f., NJW 2006, 1971 = BGHZ $166,369$.

462 Liegt beispielsweise ein Kaufvertrag vor, so sind Kaufgegenstand, Kaufpreis und Verkäufer bereits durch die invitatio ad offerendum vorgegeben, der (potenzielle) Käufer wird durch das Legitimationszeichen definiert.

463 Vgl. Sester/Nitschke, CR 2004, 548, 551; Gitter/Roßnagel, K\&R 2003, 64, 65; Cornelius, MMR 2002, 355, 356; Grapentin, S. 101 f., die aber in der Regel von Blanketterklärungen ausgehen, die weiter unten noch genauer dargelegt werden. 
möglicherweise ein Wille kundgetan werden könnte. ${ }^{464}$ Es liegt deshalb im Tatsächlichen eine Divergenz zwischen Wille und Erklärung vor. Um dem $\mathrm{zu}$ begegnen, wird teilweise vertreten, dass sich der Handlungswille schon in der Inbetriebnahme des autonomen Systems zeige. ${ }^{465}$ Sofern dadurch das Merkmal des Handlungswillens erfüllt werden sollte, müsste diese Handlung auch schon objektiv als Willenserklärung aufgefasst werden können. Die Handlung die Folge des Handlungswillens ist, ist die Handlung, die das Erklärungszeichen setzt. ${ }^{466}$ Sie ist somit kausal für die Erklärung. Folglich muss schon die Inbetriebnahme die objektive Voraussetzung einer Willenserklärung erfüllen. Es kann aber bezweifelt werden, dass ein objektiver Dritter per se in der Inbetriebnahme einen kundgetanen Willen erkennen würde, der eine rechtliche Folge herbeiführen soll, die durch die Künstliche Intelligenz bzw. durch das autonome System realisiert werden soll und somit für sich genommen schon eine Erklärung wäre. Anders wäre es möglicherweise dann, wenn entweder mit der Inbetriebnahme explizit Handlungen vollführt werden, die auf entsprechende Erklärungserzeugung schließen lassen, beispielsweise die Hinterlegung von Legitimationszeichen und entsprechende Konfiguration des Systems für Bestellungen womit gleich auch für gewisse Erklärungen das Erklärungsbewusstsein vorliegt - oder der Zweck des Systems keinen anderen Schluss zulässt. Sobald das autonome System für einen anderen Zweck in Betrieb genommen wird, reicht eine reine Inbetriebnahme in keinem Fall aus, möglicherweise sind dann weitere Akte notwendig. ${ }^{467}$

Daraus folgt, dass per se keine Handlung vorliegt, die eine Erklärung zur Folge hat. Ein möglicherweise bestehender Handlungswille zum Zeitpunkt der Inbetriebnahme kann keine spätere Erklärung begründen, wenn nicht die Handlung selbst Erklärung ist, denn »[h]andelt der Erklärende (und das sogar mit Rechtsbindungswille), ohne dass dies aus objektiver Sicht erkennbar wäre, [...] fehlt es von vornherein an einer wirksamen Willenserklärung «. ${ }^{468}$ Wird für gewisse Konstellationen bejaht, dass die Inbetriebnahme bereits objektiv eine Willenserklärung ist und entsprechender

464 Aus diesem Grund gehören Systeme, die aufgrund der nutzerseitigen Programmierung ein bestimmtes Produkt immer nachbestellen, nicht zu dieser Kategorie.

465 Spindler/Schuster/Spindler, Vorb. zu $§ \S 116$ ff. BGB Rn. 6; allgemein auch Cornelius, MMR 2002, 353, 355.

466 Vgl. Bork, Rn. 589.

467 Beispielsweise ist der (primäre) Zweck eines Kühlschranks mit autonomer Bestellfunktion zunächst auch nur das Kühlen von körperlichen Gegenständen.

468 BeckOGK/Rehberg, § 119 BGB Rn 231. 
Handlungswille vorliegt, so ist weiter $\mathrm{zu}$ fragen, ob ein Erklärungsbewusstsein durch den Benutzer vorhanden ist. In der Regel wird in den verbleibenden seltenen Fällen ein Benutzer die Inbetriebnahme eines autonomen Systems nicht als rechtserhebliche Handlung verstehen, die als Erklärung aufgefasst werden kann, selbst wenn er entsprechende Handlungen vornimmt, die objektiv diesen Schluss zulassen könnten, wie die Hinterlegung eines Legitimationszeichens. Sieht man von der problematischen Konstellation eines »allgemeinen Erklärungswillens ${ }^{469} \mathrm{ab},{ }^{470}$ dann verbleibt die Frage, ob der Benutzer bei Inbetriebnahme mit der im Verkehr üblichen Sorgfalt hätte erkennen und vermeiden können, das sein Verhalten als Erklärung aufgefasst werden kann. ${ }^{471}$ Bejaht man dies, würde auch die Problematik des fehlenden Abgabewillens gelöst, da die Erklärung dann zumindest fahrlässig in den Verkehr gelangt wäre. Dennoch dürfte eine Erklärungsfahrlässigkeit nur in wenigen Fällen anzunehmen sein, da auch mit der im Verkehr üblichen Sorgfalt bei einem autonomen System nicht erkennbar ist, dass man mit der Inbetriebnahme rechtserheblich handelt. Nur bei sehr speziellen autonomen Systemen, die einerseits speziell dem Zweck der Bestellungen dienen und andererseits durch Verkehrskreise mit besonderen Kenntnissen ${ }^{472}$ über dieses System betrieben werden, kann derzeit angenommen werden, dass der Benutzer mit der in seinem Verkehrskreis üblichen Sorgfalt hätte erkennen und vermeiden können, dass eine rechtserhebliche Erklärung vorliegt.

Mit zu berücksichtigen ist, dass bei der Vorverlagerung des Rechtsbindungswillens auf die Inbetriebnahme möglicherweise ein Wertungswiderspruch zu der Begründung der invitatio ad offerendum besteht. Bei dieser soll der Anbietende, der sich an ein unbestimmtes Publikum richtet, gerade vor dem Abschluss gewisser Rechtsgeschäfte geschützt werden; insbesondere davor, sich seinen Vertragspartner nicht aussuchen zu können, dessen Leistungsfähigkeit nicht einschätzen zu können oder selbst nicht erfüllen zu können. ${ }^{473}$ Genau dies kann bei autonom abgegebenen Erklärungen aber geschehen: Das System, das eine große Anzahl von potenziellen Vertragspartnern zur Auswahl hat, kann beispielsweise Anbieter wählen, die der Benutzer ablehnt oder gar Waren bestellen, die außerhalb der finanziellen Möglichkeiten des Benutzers liegen.

469 Vgl. Spindler/Schuster/Spindler, Vorb. zu $§ 116$ ff. BGB Rn. 6 m.w.N.

470 Ebenso kritisch Teubner, AcP 218 (2018), 155, 179 ff.

471 S. o., Dichotomie von Wille und Erklärung (S. 83).

472 In der Regel wohl nicht der Durchschnittsverbraucher.

473 Staudinger/Bork, $§ 145$ BGB Rn. 3 f. 
2. Annahmen durch autonome Systeme

Denkbar ist auch, dass das autonome System dem Zweck der Annahme von Angeboten dient. Im Unterschied zu autonom begründeten Annahmeerklärungen, werden bei automatisierten Systemen die Angebote ungeprüft oder nur nach vorgegebenen Regeln geprüft, aus einem Vorrat von vordefinierten Annahmeerklärungen übertragen. ${ }^{474}$ Dagegen entscheidet ein autonomes System aus den ihm zur Verfügung stehenden Informationen über den potenziellen Vertragspartner - beispielsweise über die Bonität eigenständig und nicht durch vollständig vorgefasste Regeln, ob die Annahme erklärt werden soll. Da auch in diesem Fall der Annehmende nicht das autonome System selbst ist, sondern der Benutzer, muss das Annahmeverhalten des autonomen Systems dem Benutzer zuzurechnen sein.

Damit überhaupt eine Annahme erklärt werden kann, muss ein wirksames Angebot vorliegen, das heißt - als empfangsbedürftige Willenserklärung - dem Erklärungsempfänger, also dem Benutzer zugegangen sein. ${ }^{475} \mathrm{Da}$ aber das Angebot dem autonomen System zugeht, ist fraglich, ob dies rechtlich als Zugang einer Willenserklärung zu werten ist, ob also der Zugang dem Benutzer zugerechnet werden kann. Unter Berücksichtigung der Debatte über die (Teil-)Rechtsfähigkeit von autonomen Systemen ist hier zu diskutieren, ob - im Gegensatz zu den bisher betrachteten Fällen - ein Angebot unter Anwesenden vorliegt, da das autonome System sofort nach Zugang reagieren könnte ${ }^{476}$ und bei einer (Teil-)Rechtsfähigkeit das Angebot mit Zugang zum autonomen System sogar tatsächlich zugegangen wäre. Da die Erklärung aber durch ihre Speicherung von gewisser Dauer dennoch verkörpert ist, würde der Zugang auch in diesem Fall eintreten, wenn die Erklärung im Machtbereich des Empfängers liegt ${ }^{477}$ - unabhängig davon, ob der Empfänger der Benutzer oder das autonome System selbst ist - sodass diese Frage zunächst rein akademischer Natur zu sein scheint. ${ }^{478}$ Relevanter Unterschied von verkörperten Willenserklärungen unter Abwesenden zu verkörperten Willenserklärungen unter Anwesenden ist, dass bei Letzteren Abgabe und Zugang

474 S. o., Automatische Annahmen (S. 97).

475 Es wird an dieser Stelle davon ausgegangen, dass eine (zurechenbare) Willenserklärung vorliegt; auf die Situation, dass auf beiden Seiten autonome Systeme agieren, wird weiter unten in diesem Abschnitt eingegangen,

476 Vgl. Grapentin, S. 105.

477 MünchKommBGB/Einsele, § 130 BGB Rn. 27.

478 Vgl. Pieper, GRUR-Prax 2019, 298, 299. 
zeitlich zusammenfallen, ${ }^{479}$ was gerade im Bereich der elektronischen Willenserklärungen nicht der Fall sein muss. ${ }^{480}$ Insoweit handelt es sich um verkörperte Willenserklärungen unter Abwesenden. Damit werden entsprechende Angebote gem. $\S 130$ Abs. 1 S. 1 BGB wirksam, wenn sie in den Machtbereich des Empfängers geraten und gewöhnlicherweise mit ihrer Kenntnisnahme zu rechnen ist. Das autonome System ist eine Empfangseinrichtung, gleich einem Briefkasten. ${ }^{481}$ Daher bestimmt sich auch die Annahmefrist nach $\S 147$ Abs. 2 BGB, wonach auf die gewöhnlichen Umstände - die denen in der elektronischen Kommunikation entsprechen abzustellen ist. ${ }^{482}$ Nähme man für Diskussionszwecke an, dass die Willenserklärung unverkörpert ist, würde eine solche Annahme auch der Natur des autonomen Systems und damit der Künstlichen Intelligenz nicht gerecht werden. Die Künstliche Intelligenz kann eine Erklärung nicht in dem Sinne falsch verstehen wie ein Mensch: Entweder ist sie im Übertragungsweg verfälscht worden, was möglicherweise nicht erkannt wurde, oder das Fehlermoment liegt im Verhalten der Künstlichen Intelligenz, was aber durch ihre deterministische Natur bei dieser Eingabe derart immer wieder auftreten würde.

Liegt ein wirksames Angebot vor, so muss die (erzeugte) Annahmeerklärung auch Willenserklärung sein, also die Voraussetzungen an eine Willenserklärung erfüllen. Ebenso wie bei erzeugten Angeboten liegt das Problem im subjektiven Tatbestand: Zunächst muss auch hier überhaupt eine Handlung des Benutzers gefunden werden, mit der sich der Handlungswille begründen lässt. Hinzu kommt, dass entweder Erklärungsbewusstsein oder Erklärungsfahrlässigkeit vorliegen muss. Im Gegensatz zu automatisierten Bestellsystemen - die in der Regel Angebote abgeben ${ }^{483}$ - lässt sich bei den autonomen Systemen, die nur Annahmen erzeugen, das Vorliegen der notwendigen subjektiven Voraussetzungen begründen, zumindest dann, wenn sie den automatischen Systemen, die Annahmen erzeugen und übertragen, im Ergebnis gleichen. Beim automatischen System wird jedes Angebot - nach durch den Menschen vordefinierten Regeln -

479 Neuner, § 33 Rn. 27.

480 S. o., Zugang (S. 93).

481 Pieper, GRUR-Prax 2019, 298, 299; Gitter/Roßnagel, K\&R 2003, 64, 66 f.; Grapentin, S. $103 \mathrm{f}$.

482 Vgl. Cornelius, MMR 2002, 353, 357 der annimmt, dass sich die Zeit auf »nahezu Null« reduziert und daher kein Unterschied mehr zu $\S 147$ Abs. 1 BGB besteht.

483 Geht man davon aus, dass keine invitatio ad offerendum vorliegt, so würde das bestellende, autonome System die Annahme erklären, wobei die Problematik dieses Systems bei der Annahme dann der im vorherigen Abschnitt beschriebenen Problematik des Willens beim Angebot gleicht. 
angenommen, welche allerdings verhältnismäßig simpel sind. Das autonome System entscheidet im Unterschied dazu nach praktisch nicht vorhersehbaren und nicht zwangsläufig nachvollziehbaren, aber deterministisch begründeten Regeln. Im Endeffekt sind dies nur viel komplexere und nicht mehr (vollständig) durch den Benutzer vordefinierte, sondern antrainierte Regeln. Der allgemeine Wille zur Annahme von Erklärungen an sich ist aber in beiden Fällen vorhanden. Unschädlich ist dabei auch, ob die entsprechende Annahme oder Nichtannahme gewollt ist. Entweder erklärt das autonome System die Annahme gegenüber einem Vertragspartner, dem gegenüber nicht angenommen werden soll, was allerdings ein Problem des nicht konstitutiven Geschäftswillens ist oder das autonome System erklärt keine Annahme gegenüber jemandem, gegenüber dem eine Annahme eigentlich gewollt ist. Bei Letzterem kann die Annahme - sofern sie noch fristgerecht ist-immer noch erklärt werden, im Zweifel konkludent mit der Leistungserbringung. Auch ein Problem mit der Abgabe besteht insoweit nicht. ${ }^{484}$ Daraus folgt, dass - wenn man bereits bei einem automatischen System bejaht, dass die Annahmeerklärungen schon mit der Inbetriebnahme vorliegen und nur später übertragen werden - es als argumentum a fortiori auch für autonome Systeme gelten müsste, die Erklärungen erzeugen und übertragen, jedenfalls in dem Fall, in welchem das autonome System die gleiche Tätigkeit vollführt. Es darf aber nicht außer Acht gelassen werden, dass ein solches System wahrscheinlich eine sehr starke Vereinfachung der Realität darstellt und die Annahme des Erst-Recht-Schlusses nur dann gelten kann, wenn der einzige Zweck dieser Systeme die Erzeugung einer Annahme ist.

Aus den bisherigen Darlegungen zu autonomen Systemen folgt aber, dass die Situation dann gänzlich anders zu bewerten ist, wenn das autonome System keine Annahme - als einfaches »Ja« - erklärt, sondern verhandelt. Dann wäre die modifizierte Annahme gem. $\S 150$ Abs. 2 BGB als neues Angebot zu sehen, was - wie dargelegt - bei der Zurechnung des Willens zum Benutzer problematisch ist. Hier entstehen die gleichen Probleme, wie bei den autonomen Angeboten. Daraus folgt auch, dass die Situation sogar gänzlich problematisch wird, wenn auf beiden Seiten autonome Systeme agieren. Am Vertragsschluss ist dann keine menschliche Beteiligung mehr vorzufinden.

484 Möglicherweise könnte ein Fall des $§ 151$ S. 1 BGB vorliegen, der allerdings bei Unternehmern, die ein autonomes System zur Annahme betreiben, wahrscheinlich an $\S 312$ i Abs. 1 Nr. 3 BGB scheitert, auch wenn diese Bestätigung nicht per se als Annahme zu verstehen ist, s. o., Automatische Annahmen (S. 97). 


\section{Vorliegen einer Blanketterklärung}

Eine teilweise diskutierte Lösung könnte darin liegen, dass eine Blanketterklärung des Benutzers vorliegt. ${ }^{485}$. Dabei handelt es sich um eine Erklärung eines Blankettgebers, deren Inhalt durch einen Dritten vervollständigt wird. ${ }^{486}$ Den Befürwortern nach macht es keinen Unterschied, ob die Erklärung von einem Menschen oder einer Maschine vervollständigt wird. ${ }^{487}$ Zwar würde sich das Problem der Abgabe der Willenserklärung lösen, ${ }^{488}$ dennoch muss hier ein generelles Erklärungsbewusstsein fingert werden, ${ }^{489}$ da die inhaltslose Blanketterklärung auch den Voraussetzungen einer Willenserklärung genügen muss. Weiterhin besteht ein bedeutsamer Unterschied zum Blankett in Papierform: Wird einem Dritten ein Blankett zur Vervollständigung übergeben, ist dem Erklärenden allein durch dessen Form als Urkunde bewusst, dass er ein leeres Blankett aus der Hand gibt. Es besteht eine Warnfunktion der Urkunde. In diesem Fall liegen auch Rechtsbindungswille, Handlungsbewusstsein und Erklärungsbewusstsein vor. Im Gegensatz dazu wäre die Inbetriebnahme einer autonom agierenden Künstlichen Intelligenz die Erstellung und Weitergabe von unendlich vielen leeren Blanketten, ohne dass dies dem Benutzer - durch die warnende Form des eigentlichen Blanketts - bewusst wird. Im Weiteren fehlt es hier gänzlich an den Voraussetzungen an eine Willenserklärung.

\section{Zwischenergebnis}

Bei autonomen Systemen besteht insbesondere bei autonomen Angeboten ein schwer überbrückbares Defizit von Willen und Erklärung, welches bei der derzeitigen Willenserklärungsdogmatik keine wirksame Willenserklärung begründet. Gewiss ist an dieser Lösung unbefriedigend, dass auch bei abgegebenen Erklärungen der Künstlichen Intelligenz, die dem Willen des Benutzers entsprechen, keine andere Handlung des Benutzers infrage kommt, um die subjektiven Voraussetzungen an eine Willenserklärung und damit eine Willenserklärung zu begründen.

485 Sester/Nitschke, CR 2004, 548, 549 f.; Gitter/Roßnagel, K\&R 2003, 64, 66; Köhler, AcP 182 (1982), 126, 134; Reusch/Weidner, Rn. 44 ff.; Grapentin, S. 100.

486 Vgl. MünchKommBGB/Schubert, § 172 BGB Rn. 2 ff.

487 Grapentin, S. 92.

488 Grapentin, S. $101 \mathrm{f}$.

489 Vgl. Wiebe, S. $156 \mathrm{f}$. 


\section{Folgen}

Wird Künstliche Intelligenz nur als Hilfsmittel zur Übertragung oder Verkörperung von Willenserklärungen verwendet, so bestehen keine Unterschiede zu herkömmlichen technischen Einrichtungen, weder was die Erzeugung der Erklärung angeht noch im Hinblick auf deren Wirksamkeit. Die Willenserklärungsdogmatik mit ihrem Dualismus aus Willen und Erklärung weist insoweit keine KI-spezifischen Herausforderungen auf. Wird eine Erklärung erzeugt, kann sie - als eigene Erklärung des Benutzers diesem zugerechnet werden. Sind die notwendigen subjektiven Voraussetzungen einer Willenserklärung erfüllt, so liegt eine Willenserklärung vor; andernfalls greift eine Rechtsscheinhaftung. Etwaige Irrtümer aufseiten des Benutzers berechtigen evtl. zur Anfechtung. Daraus folgt zugleich, dass eine Analogie zum Boten hier nicht kategorisch auszuschließen ist, da die Willensbildung vollständig aufseiten des Benutzers liegt. Sieht man die Künstliche Intelligenz als Einrichtung im Sinne des § 120 BGB und stimmt die durch die Künstliche Intelligenz übertragene Erklärung nicht mit dem kundgetanen Willen des Benutzers überein, so eröffnet dies dem Benutzer eine Anfechtungsmöglichkeit; denn der Wille des Benutzers ist durch die Künstliche Intelligenz nur fehlerhaft übertragen worden; ein »eigener« Wille der Künstlichen Intelligenz liegt nicht vor. Dies gilt unabhängig davon, ob die Künstliche Intelligenz nun fehlerhaft ist oder unvorhersehbares Verhalten gezeigt hat. Grundsätzlich ist der Problematik der fehlerhaften Übertragung durch eine technische Einrichtung bisher wenig Aufmerksamkeit geschenkt worden, obwohl sie in Kommunikationsnetzen recht oft vorkommt, auch wenn sie korrigiert wird (z.B. durch Prüfsummen). ${ }^{490}$

Bei einer vollständig autonomen Erklärung einer Künstlichen Intelligenz ohne weitere (konkrete) Handlung des Benutzers - durch sog. autonome Systeme - kommt die bisherige Willenserklärungsdogmatik in gewissen Fällen an ihre Grenzen. Das gilt unabhängig davon, ob die Erklärung mit diesem Inhalt - im Nachhinein - gewollt oder ungewollt war. Damit liegt hier ein Novum gegenüber bisherigen technischen Phänomenen vor. Zwar liegt objektiv betrachtet eine Erklärung des autonomen Systems vor, diese lässt sich aber in der Regel nicht auf einen Willen des Benutzers zurückführen. Folglich sind bei den erzeugten Erklärungen der Künstlichen Intelligenz die notwendigen subjektiven Tatbestandsmerkmale nicht erfüllt;

490 Vgl. zu den unterschiedlichen Übertragungsmöglichkeiten in verteilten Systemen und deren Korrektur, Tanenbaum/Wetherall, S. $244 \mathrm{ff}$. 
ergo besteht kein Wille des Benutzers. Zudem liegt außer durch die Inbetriebnahme des Systems keine Handlung vor, die objektiv Erklärung sein kann, sodass die Debatte um mögliche subjektive Merkmale bei der Inbetriebnahme keine Rolle spielt. Ausnahmen lassen sich nur in seltenen Fällen bei bestimmten autonomen Systemen begründen, die zweckgerichtet in Betrieb genommen und konfiguriert werden, insbesondere bei autonomen Systemen, die Annahmen erzeugen.

Daraus folgt, dass - sofern man am Merkmal des Willens als Voraussetzung einer Willenserklärung festhält - derartige Maschinenerklärungen derzeit nicht als Willenserklärung aufzufassen sind und daher schon nicht zugerechnet werden können. Da dies in vielen Fällen des Vertragsschlusses für den Erklärungsempfänger nicht erkennbar ist und dieser in Folge einer derartigen Erklärung dennoch an den Benutzer der Künstlichen Intelligenz leistet, entsteht somit eine condictio indebiti (§ 812 Abs. 1 S. 1 Alt. 1 BGB). Ist der Leistungsempfänger Verbraucher und wurden bewegliche Sachen an ihn geliefert, steht dem Anspruch aus $\S 812$ Abs. 1 S. 1 Alt. 1 BGB auch nicht $\S 241$ a Abs. 1 BGB entgegen. Gem. $\S 241 \mathrm{a}$ Abs. 2 Alt. 2 BGB ist die Lieferung in der irrigen Vorstellung einer Bestellung erfolgt und der Benutzer der Künstlichen Intelligenz hätte zumindest bei Empfang der Lieferung erkennen können, dass eine irrige Bestellung vorliegt, ${ }^{49}$ die (zumindest wahrscheinlich) auf seine Künstliche Intelligenz zurückzuführen ist. Eine mögliche Lösung bei Angeboten bestünde darin, Erklärungen einer Künstlichen Intelligenz nur als Aufforderung für die Gegenseite aufzufassen, ein bestimmtes Angebot mit den Modalitäten der Erklärung der Künstlichen Intelligenz abzugeben. Der Benutzer der Künstlichen Intelligenz könnte das Rechtsgeschäft dann nach dem Angebot der Gegenseite durch die Annahmeerklärung bestätigen. Freilich würde dies eine gewisse Transparenz gegenüber dem Empfänger, der durch die Künstliche Intelligenz erzeugten Erklärung, voraussetzen. Ihre Grenzen findet diese Lösung aber auch dann, wenn auf der anderen Seite keine Künstliche Intelligenz agiert.

Geht man davon aus, dass einer Künstlichen Intelligenz zumindest eine Teilrechtsfähigkeit zukommt, die so weit geht, dass eine Künstliche Intelligenz als Hilfsperson agieren kann, bestünde die Möglichkeit, dass die Regelungen der Stellvertretung auf solche Maschinenerklärungen - zumindest in Teilen - anwendbar wären. ${ }^{492}$ Dies würde das Problem des

491 Vgl. BeckOK/Sutschet, § 241a BGB Rn. 11.

492 S. Fn. 325. 
fehlenden objektiven Tatbestandsmerkmals zum Zeitpunkt der Inbetriebnahme lösen, da es nun auf die Inbetriebnahme bzw. ein dementsprechendes Handeln des Benutzers nicht mehr ankommt. Damit kann die erzeugte Erklärung des Systems selbst als die Erklärung herangezogen werden, die dann zumindest objektiv den Erklärungstatbestand erfüllt.

Allerdings setzt die Stellvertretung nach wie vor eine vollständige eigene Willenserklärung der Hilfsperson voraus, die bei einer Künstlichen Intelligenz problematisch ist. Einer Künstlichen Intelligenz fehlt per se der (subjektive) Wille, sodass sie niemals Erklärungen erzeugen kann, die eigene Willenserklärungen wären, sofern der Wille weiter Voraussetzung hierfür ist. Teubner nimmt an, dass - zumindest bei der Stellvertretung nur noch objektive Kriterien herangezogen würden, um eine Erklärung als (eigene) Willenserklärung zu klassifizieren und der Aspekt der eigentlichen Willensbildung nur noch eine untergeordnete Rolle spiele. Es seien nur bestimmte soziale Normen von Relevanz, die man in ein Computerprogramm »übersetzen« könne, selbstlernende Agenten könnten dies gar von selbst tun. ${ }^{493} \mathrm{Ob}$ dies dem technisch Möglichen entspricht, darf aber derzeit bezweifelt werden; Künstliche Intelligenz ist viel konkreter. Vielmehr müsste die Künstliche Intelligenz bei Änderung der sozialen Normen angepasst werden. Ein künstliches neuronales Netz müsste jedes Mal neu angelernt werden, teilweise gar angepasst. Insofern verbleibt auch diese Aufgabe beim Menschen. Insofern würde auch eine Teilrechtsfähigkeit unter pragmatischen Gesichtspunkten kein »Mehr« bei autonomen Erklärungen durch Künstliche Intelligenz mit sich bringen. Die Herausforderung bei autonomen Erklärungen durch Künstliche Intelligenz liegt somit nicht bei der Zuordnung, sondern bei den notwendigen Voraussetzungen an eine Willenserklärung. Würde man vom subjektiven Willenserfordernis bei Willenserklärungen absehen und nur noch den objektiven Erklärungstatbestand voraussetzen, so könnte eine Willenserklärung der Künstlichen Intelligenz allein durch das Legitimationszeichen zugeordnet werden. Da das autonome System auch für einen entsprechenden Zweck betrieben wird, ist es sachgerecht, das damit einhergehende Risiko diesem Benutzer auch zuzurechnen. ${ }^{494}$ Der (Teil-)Rechtsfähigkeit bedarf es daher zumindest in der Rechtsgeschäftslehre nicht; sie bietet in keinem Fall einen Mehrwert.

493 Teubner, AcP 218 (2018), 155, $183 \mathrm{f}$.

494 Ebenso Foerster, ZfPW 2019, 418, 427; Sosnitza, CR 2016, 764, 767; für automatische Erklärungen vgl. Wiebe, S. 216 ff. 


\section{Haftungsrechtliche Verhaltens- und Handlungszurechnung}

Festgestelltes Hauptmerkmal von Künstlicher Intelligenz ist es, gewisse Handlungen, die bisher primär dem Menschen obliegen, zu vollführen. ${ }^{495}$ Für die Zurechnung des haftungsrelevanten Verhaltens können dabei mehrere mögliche Kategorien gebildet werden, in denen Künstliche Intelligenz verwendet werden kann und die die möglichen Konstellationen abdecken: Erstens als Dienstleistung, die durch oder mithilfe einer Künstlichen Intelligenz angeboten wird, zweitens als Hilfsmittel in der Herstellung oder Entwicklung von Produkten und drittens als Bestandteil eines Produktes selbst. In allen diesen Fällen zeigt eine Künstliche Intelligenz zumindest nach außen - ein Verhalten, welches auch vom Menschen gezeigt oder zumindest verursacht werden kann. Zivilrechtlich fraglich ist, welche Rechtsfolgen dies auslöst. Eine besondere Steuerungsfunktion von menschlichem Verhalten kommt hier dem Haftungsrecht zu. ${ }^{496}$ Haftung ist dabei das Einstehenmüssen für eigenes oder fremdes Verhalten oder anders ausgedrückt eine Verantwortlichkeit für das (rechtswidrige) Verhalten aus einer Pflicht - sei es gegenüber jedermann oder durch eine vertragliche Pflicht gegenüber bestimmten Personen - verbunden mit evtl. Verpflichtungen zu einer Leistung. ${ }^{497}$

Somit stellt sich die Frage, ob ein Unterschied zu bisherigen haftungsrechtlich relevanten Verhaltensweisen besteht und ob es einen Unterschied macht, wenn diese Verhaltensweisen statt vom Menschen, von Künstlicher Intelligenz ausgehen. Eine wesentliche Bedeutung kommt dabei auch der Frage zu, worauf das Verhalten zurückzuführen ist, also ob es auf einem Fehler oder auf nicht vorhersehbarem Verhalten basiert. ${ }^{498}$ Im Haftungsrecht geht es dabei um die Fälle, bei welchen das Verhalten von einem Soll abweicht (Pflichtverletzung), sei es durch ein vertragliches Soll (Leistungspflichten) oder ein anderes allgemeines von der Rechtsordnung vorgegebenes Soll (weitere Verhaltenspflichten), also die Haftung aus Vertrag und Delikt. ${ }^{499}$ Gewiss kann sich das haftungsbegründende Verhalten bei Vertrag und Delikt unterscheiden, dennoch kann in beiden Fällen - wenn

495 S. o., Definitionsversuche (S. 30) und Zwischenergebnis (S. 68).

496 Vgl. Kötz/Wagner, Rn. 63.

497 Larenz, Lehrbuch des Schuldrechts I, S. 22.

$498 \mathrm{Zu}$ dieser Unterscheidung, s. o., Vom Sein und Sollen (S. 64).

499 Vgl. Foerster, ZfPW 2019, 418, 430 ff.; Jakl, MMR 2019, 711, 713 f.; Teubner, AcP 218 (2018), 155, $158 \mathrm{f}$. 
eine Haftung bejaht wird - das haftungsbegründende Ereignis auf ein Verhalten zurückgeführt werden. Möglicherweise bestehen hier aber Unterschiede zu anderen technischen Einrichtungen. ${ }^{500}$

Das Deliktsrecht ( $\S 823 \mathrm{ff}$. BGB) ist als Recht der unerlaubten Handlung schon von der Bezeichnung her verhaltensbezogen. ${ }^{501}$ Die Gefährdungshaftung ist dagegen zwar eine Risikozurechnung für eine abstrakte Gefahr, ${ }^{502}$ allerdings für eine Gefahr, die von einer Entität ausgeht, deren Risiko zugerechnet werden soll. Eine solche Entität kann aber nur gefährlich sein, wenn sie sich gefährlich verhalten kann. Aus der Schaffung oder Unterhaltung einer Gefährdung in diesem Sinne resultiert ein - wenn auch nicht per se rechtswidriges - Verhalten.$^{503}$ Auch im Vertragsrecht lässt sich - bei Pflichtverletzung - grundsätzlich eine Rückführung auf ein Verhalten des Schuldners begründen, sofern ein weiter Verhaltensbegriff zugrunde gelegt wird. ${ }^{504}$ In diesem Fällen ist es auch denkbar, dass das vertragswidrige Verhalten zugleich auch unerlaubte Handlung ist. ${ }^{505}$

Da Haftungstatbestände eine Pflicht begründen, kann nur haften, wer Träger von Rechten und Pflichten ist. Somit muss das Verhalten von Künstlicher Intelligenz als Zurechnungsausgangssubjekt einem rechtsfähigen $\mathrm{Zu}-$ rechnungsendsubjekt zugerechnet werden. Sofern man eine (Teil-)Rechtsfähigkeit von Künstlicher Intelligenz bejaht, bestünde die Möglichkeit, das Verhalten der Künstlichen Intelligenz ihr selbst zuzurechnen; andernfalls muss ein anderes und rechtsfähiges Zurechnungsendsubjekt gefunden werden. Bei fremdem Verhalten ist die haftungsrechtlich relevante Verhaltenszurechnungsnorm $\S 278$ BGB; ${ }^{506}$ im Deliktsrecht besteht die gesonderte Vorschrift des $\S 831 \mathrm{BGB}$, die zwar kein fremdes, sondern eigenes Verhalten zurechnet, ${ }^{507}$ allerdings die gleiche Funktion hat. ${ }^{508}$

Daraus folgen drei im Weiteren zu untersuchende Konstellationen: Erstens die Haftung von Künstlicher Intelligenz für ihr eigenes Verhalten (I.).

500 Vgl. Brüggemeier, AcP 219 (2019), 771, 812 ff.

501 Vgl. MünchKommBGB/Wagner, $§ 823$ BGB Rn. 63 f.; Canaris, VersR 2005, $577 \mathrm{ff}$.

502 Larenz/Canaris, Lehrbuch des Schuldrechts II/2, S. 605.

503 Vgl. zu den menschlichen Verhaltensanreizen MünchKommBGB/Wagner, Vor $\S 823$ BGB Rn. 19; Larenz/Canaris, Lehrbuch des Schuldrechts II/2, S. 608.

504 Beispielsweise, wenn das Nichtleistenkönnen als Verhalten angesehen wird, vgl. MünchKommBGB/Ernst, § 280 BGB Rn. 16 f.

505 Kötz/Wagner, Rn. 10

506 MünchKommBGB/Grundmann, § 278 BGB Rn. 1.

507 Ebenso Staudinger/Bernau, § 831 BGB Rn. 28; Buck, S. 106 in Fn. 15.

508 BeckOGK/Spindler, § 831 BGB Rn. 1 f. 
Zweitens die Haftung von beteiligten Rechtssubjekten für ihr eigenes Verhalten bzw. menschliches Verhalten, das ihnen selbst zugerechnet wird und sich durch die technische Einrichtung »Künstliche Intelligenz« verwirklicht (II.). Dieser Fall gleicht den herkömmlichen technischen Einrichtungen. Drittens die Haftung von beteiligten Rechtssubjekten für das Verhalten der Künstlichen Intelligenz, das den beteiligten Rechtssubjekten zugerechnet wird (III.). Gemeinsam haben diese drei Fälle, dass das Verhalten für den Schaden kausal sein muss, sodass haftungsbegründende und haftungsausfüllende Kausalität am Ende gemeinsam für alle Konstellationen untersucht wird (IV.).

\section{Haftung der Künstlichen Intelligenz für eigenes Verhalten}

Nimmt man an, dass Künstliche Intelligenz Träger von Rechten und Pflichten sein kann, so könnte eine Künstliche Intelligenz Haftungsschuldner sein und somit für eigenes Verhalten haften. Betrachtet man die Zustandsänderung der Künstlichen Intelligenz, aus der ihr Verhalten resultiert, ${ }^{509}$ so kommt es zunächst nicht darauf an, ob es deterministisch oder nicht-deterministisch begründet ist. Liegt in diesem Verhalten eine Pflichtverletzung, so besteht objektiv kein Unterschied, ob der Grund der Pflichtverletzung in menschlichem oder maschinellem Verhalten liegt. Das deutsche Zivilrecht basiert im Grundsatz aber auf dem Verschuldensprinzip. ${ }^{510}$ Der Schuldner haftet für die Pflichtverletzung, aber im Regelfall nur dann, wenn er sie verschuldet hat. Den Schuldner muss somit für eine vertragliche oder deliktische Haftung ein Verschulden treffen. ${ }^{511}$ Im Zivilrecht setzt das ein subjektiv vorwerfbares Verhalten voraus. ${ }^{512}$ Sofern sich aus dem Schuldverhältnis nichts anderes ergibt, ist dies der Fall, wenn sich der Schuldner vorsätzlich oder fahrlässig verhalten hat (§ 276 Abs. 1 BGB). Daraus folgt, dass eine Künstliche Intelligenz - selbst für den Fall, dass sie rechtsfähig wäre - nur dann für eigenes Verhalten haften kann, wenn sie ein Verschulden treffen kann, sich also vorsätzlich oder fahrlässig verhalten kann. ${ }^{513}$

509 S. o., Problematik des Vergleichs zum Menschen (S. 26).

510 Vgl. Deutsch, AcP 202 (2002), 889, 892 f.

511 Brox/Walker, Allg. SchuldR, § 20 Rn. 1.

512 Palandt/Grüneberg, § 276 BGB Rn. 5.

513 Der Fall, dass eine Künstliche Intelligenz eine Gefahr schafft oder unterhält und somit Haftungsschuldner einer (verschuldensunabhängigen) Gefährdungshaftung 
Bereits die Tatsache, dass Künstliche Intelligenz deterministisch ist, schließt die Verschuldensfähigkeit aber a priori aus; ${ }^{514}$ durch ihren Determinismus bestehen keine möglichen Handlungsalternativen für die Künstliche Intelligenz, vielmehr gibt es in der jeweiligen konkreten Situation nur das gezeigte Verhalten. Es bestehen auch - unabhängig vom Autonomiegrad - keine kognitiven oder kognitiv-ähnlichen Fähigkeiten, die ein eigenes Verschulden ermöglichen. ${ }^{515}$ Im Einzelnen: Vorsatz als Wissen und Wollen des rechtswidrigen Erfolges setzt voraus, dass der Haftungsschuldner die rechtswidrige Handlung auch gewollt hat, ${ }^{516}$ was Künstliche Intelligenz sowohl mangels eigenen Willens als auch darauf bezogenem Wissens nicht kann. Fahrlässig i.S.d. § 276 Abs. 2 BGB handelt, »wer die im Verkehr erforderliche Sorgfalt außer Acht lässt«. Im Rahmen dessen wird jedoch ein objektiver Sorgfaltsmaßstab zugrunde gelegt, welcher sich an einem durchschnittlichen Angehörigen des maßgeblichen Verkehrskreises bemisst. ${ }^{517}$ Ferner muss der schädigende Erfolg für den Schuldner auch voraussehbar und vermeidbar sein. ${ }^{518}$ Das bedeutet im Umkehrschluss, dass schon dann kein Verschulden vorliegen kann, wenn der Erfolg bis zum Zeitpunkt des schädigenden Ereignisses nicht erkennbar gewesen ist. Daran fehlt es der Künstlichen Intelligenz, denn sie würde ihr Verhalten als solches - mangels moralischer Eigenschaften - nicht an einer Maxime messen und es daher in diesem Sinne nicht als fehlerhaft oder falsch erkennen können. Somit lernt eine Künstliche Intelligenz nicht aus falschem Verhalten wie ein Mensch. Die deterministische Eigenschaft führt dazu, dass das fehlerhafte Verhalten bei gleicher Ausgangslage immer wieder gezeigt würde. ${ }^{519}$

sein kann, wird an dieser Stelle für derart unwahrscheinlich erachtet, dass er (derzeit) keiner weiteren Untersuchung bedarf.

514 S. o., Determinismus (S. 58).

515 So aber Hacker, RW 2018, 243, 257.

516 BGH, U. v. 20.12.2011 - VI ZR 309/10, Rn. 10, NJW-RR 2012, 404; BGH, U. v. 05.03.2002 - VI ZR 398/00, NJW 2002, 1643, 1645 f.; Jauernig/Stadler, § 276 BGB Rn. 15; Palandt/Grüneberg, § 276 BGB Rn. 10; Staudinger/Caspers, § 276 BGB Rn. 22.

517 BGH, U. v. 12.07.2005 - VI ZR 83/04, NJW 2006, 1271, 1272; BGH, U. v. 11.04.2000 - X ZR 19/98, NJW 2000, 2812, 2813.

518 Palandt/Grüneberg, § 276 BGB Rn. 20 f.

519 Ebenso Wagner, in: Faust/Schäfer, S. 33. 


\section{Haftung für eigenes Verhalten der Beteiligten}

Möglich ist aber, dass das Verhalten der Beteiligten eine Haftung auslöst. Gerade weil das Verschulden der Künstlichen Intelligenz ausfällt und somit eine Haftung der Künstlichen Intelligenz für eigenes Verhalten nicht infrage kommt, ist fraglich, ob ein Verhalten der beteiligten Rechtssubjekte vorliegen kann, das haftungsrelevant ist. ${ }^{520}$ Diese Frage gleicht zunächst der Frage nach der grundsätzlichen Haftung von Beteiligten bei jeglichen technischen Phänomenen; fraglich ist aber, ob sich bei Künstlicher Intelligenz Unterschiede ergeben. Die deterministische Eigenschaft und damit auch deterministisches Verhalten von Künstlicher Intelligenz legt zwar nahe, dass das Verhalten der Künstlichen Intelligenz nur Folge des Verhaltens der Beteiligten ist; allerdings widerspricht das unvorhersehbare Verhalten und die damit verbundene begrenzte Erklärbarkeit von Künstlicher Intelligenz dieser Annahme.

\section{Verschuldensabhängige Haftung}

Hersteller, Betreiber oder Benutzer ${ }^{521}$ müssen sich neben dem objektiv pflichtwidrigen Verhalten auch subjektiv vorwerfbar (durch die Künstliche Intelligenz) verhalten haben, also mit Vorsatz oder Fahrlässigkeit gehandelt haben. Somit muss auf Grund des Verschuldensprinzips im Haftungsrecht bei einem der Beteiligten ein Verschulden vorliegen.

\section{a) Vorsatz}

In Bezug auf Künstliche Intelligenz liegt Vorsatz vor, wenn das schadenauslösende Ereignis mit Wissen und Wollen von einem der Beteiligten durch die Künstliche Intelligenz verwirklicht wurde. Insoweit ist der Vorsatz im Kontext der Künstlichen Intelligenz offensichtlich unproblematisch. ${ }^{522}$ Vorsatz liegt dann nur bei einer Manipulation oder bei einem zielgerichteten Einsatz der Künstlichen Intelligenz vor - beispielsweise des Programmes selbst oder der Trainingsdaten - mit dem Ziel mit Wissen und Wollen eine Pflichtverletzung auszulösen. Die Künstliche Intelligenz ver- 
kommt dann - wie jede andere technische Einrichtung - zum bloßen Werkzeug, sodass die gleichen Maßstäbe wie bei anderen technischen Einrichtungen anzulegen sind. ${ }^{523}$

\section{b) Fahrlässigkeit}

Erkenntnisreicher ist die Antwort auf die Frage, wann das Verhalten der Beteiligten fahrlässig ist. Da die im Vorhinein von den Beteiligten nicht erkennbaren schädigenden Ereignisse keine Fahrlässigkeit begründen, folgt daraus, dass nur noch die wahrscheinlichen Fälle betrachtet werden können. Das unvorhersehbare Verhalten einer Künstlichen Intelligenz ist im Vorhinein nicht erkennbar und daher kein Fehler, ${ }^{524}$ sodass es unterhalb der Grenze liegt, die an die gebotene Sorgfalt der Beteiligten zu stellen ist. Tritt unvorhersehbares Verhalten der Künstlichen Intelligenz auf, liegt daher keine Fahrlässigkeit mehr vor und damit auch kein eigenes Verschulden der Beteiligten. ${ }^{525}$ Offen bleibt nur die Frage, wie weit der objektive Sorgfaltsmaßstab der Beteiligten bei Fehlern reicht, welche Fälle also hätten erkannt werden können. Dies dürfte im Regelfall genauso wie bei herkömmlichen technischen Phänomenen zu beurteilen sein, also nach dem Inhalt des jeweiligen Schuldverhältnisses. ${ }^{526}$

Eine Begrenzung findet der Sorgfaltsmaßstab auch in den Anforderungen, die an die durchschnittlichen Angehörigen der einzelnen Verkehrskreise zu stellen sind. Zunächst kann zwischen den Beteiligten (Hersteller, Betreiber und Benutzer) differenziert werden, die allerdings möglicherweise in weitere Verkehrskreise zu unterteilen sein werden. Denn ist ein beteiligter Akteur nur Benutzer und nicht Betreiber, so wird in der Regel kein hoher Sorgfaltsmaßstab an den Benutzer zu stellen sein, da dieser nur eine Dienstleistung eines Betreibers in Anspruch nimmt. Ggf. wird in den Betrieb der Künstlichen Intelligenz nur wenig Einblick gewährt und die Möglichkeit von Prüfungen der Künstlichen Intelligenz kann gering ausfallen. Diese Anforderungen sind umso geringer, wenn die Künstliche Intelligenz einer großen Anzahl an Benutzern angeboten wird und umso höher, wenn die Künstliche Intelligenz vom Betreiber an die spezifischen Be-

523 Vgl. BGH, U. v. 25.05.2020 - VI ZR 252/19, Rn. 13 ff., NJW 2020, 1962.

524 Allgemein für Produktfehler schon Brüggemeier, WM 1982, 1294, 1302; Brüggemeier, ZHR 152 (1988), 511, 516 ff.

525 Ebenso Reusch/Weidner, Rn. 58.

526 Vgl. BeckOGK/Schaub, § 276 BGB Rn. 61. 
dürfnisse des Benutzers angepasst wird. Auch wird der objektive Sorgfaltsmaßstab geringer ausfallen, wenn der Benutzer Verbraucher ist. Im Gesamten dürfte sich der Sorgfaltsmaßstab der Benutzer nicht von dem der Benutzer anderer Software unterscheiden.

Unklar ist, ob der Ansatz der Benutzer auf die Betreiber einer Künstlichen Intelligenz übertragbar ist. Sofern ein Verbraucher als Endanwender ein Computersystem mit einer Künstlichen Intelligenz oder ein anderes Produkt, welches ein solches System enthält, erwirbt und es dann für eigene Zwecke betreibt, kann von ihm nicht verlangt werden, nicht offensichtliche Fehler zu erkennen. Das gilt auch, wenn bekannt ist, dass die Ware bei Fehlern potenziell gefährlich ist. Gerade einem Verbraucher kann nicht zugemutet werden, Fehler in komplexen Computersystemen oder Software zu finden. Auch bei allgemeiner Software wird vom Verbraucher das Erkennen von Fehlern nicht erwartet, wohl aber das Einspielen von Softwareupdates. ${ }^{527} \mathrm{Im}$ unternehmerischen Umfeld ist beim Betreiber danach zu differenzieren, ob die Künstliche Intelligenz dem unternehmerischen oder dem administrativen Teil zuzuordnen ist. Beim administrativen Teil sind wie schon beim Verbraucher - keine gesonderten Kenntnisse über die Künstliche Intelligenz zu erwarten, sodass die Sorgfaltsmaßstäbe denen des Verbrauchers gleichen. Anders kann dies sein, wenn die Künstliche Intelligenz für den genuin unternehmerischen Zweck verwendet wird: Wird die Künstliche Intelligenz für den Kunden des unternehmerischen Betreibers tätig, so wird ein Betreiber, der KI-bezogene Dienstleistungen - beispielsweise Erkennung von Mustern (Sprache, Gesichter etc.) - anbietet, wohl höhere Sorgfaltspflichten haben als ein reiner Benutzer. Grundsätzlich gilt auch hier: Je komplexer und spezieller eine Künstliche Intelligenz ist, umso höher muss der Sorgfaltsmaßstab des Betreibers sein, auch wenn er die Künstliche Intelligenz nur als Hilfe für seine eigentliche Tätigkeit verwendet.

Problematisch bleibt die Situation, wenn der Benutzer oder Betreiber eine Künstliche Intelligenz als Hilfsmittel in der Entwicklung oder Herstellung von Produkten verwendet, ${ }^{528}$ beispielsweise einen evolutionären Algorithmus. ${ }^{529}$ Wird durch einen Fehler in der Künstlichen Intelligenz genauer durch deren fehlerhafte Ausgabe - das (spätere) Produkt fehlerhaft, kann aber nur darauf abzustellen sein, ob der Betreiber in seiner Funktion

527 Vgl. Borges, NJW 2012, 2385, 2386.

528 Wobei angenommen wird, dass die Künstliche Intelligenz von einem Hersteller stammt und keine Eigenentwicklung ist.

529 S. o., Evolutionäre Algorithmen (S. 42). 
als Hersteller dieses Produktes den Produktfehler hätte erkennen und vermeiden können. Hier kommt dann die reguläre Produkthaftung zum Tragen. Eine Qualitätskontrolle der Künstlichen Intelligenz, beispielsweise durch Methoden der Erklärbarkeit des Explainable AI (xAI) ${ }^{530}$ kann man vom Betreiber in aller Regel nicht verlangen.

Verbleibt noch der Hersteller (der Künstlichen Intelligenz) und damit die Frage, welcher objektiver Sorgfaltsmaßstab bei Herstellern anzusetzen ist. Eine besondere Stellung nehmen hier weiterlernende Systeme ein, ${ }^{531}$ auf deren Weiterlernen der Hersteller in der Regel keinen Einfluss hat. Das Verhalten der Künstlichen Intelligenz im Weiterlernen ist bereits von vornherein nicht vorhersehbar. Daher ist es kein Fehler, sondern unvorhersehbares Verhalten. Fehler lägen hier nur dann vor, wenn der Teil der Software fehlerhaft wäre, der für das Anlernen zuständig ist. Das ist allerdings aufgrund der geringeren Komplexität dieses Teils der Künstlichen Intelligenz ein mit anderer fehlerhaften Software vergleichbares Problem.

Der objektive Sorgfaltsmaßstab des Herstellers einer Künstlichen Intelligenz beurteilt sich nach den Regeln der (verschuldensabhängigen) Produkthaftung. ${ }^{532}$ Diese kann zwar auch vertraglicher Natur sein, ${ }^{533}$ die relevanten Ansätze finden sich allerdings bei der deliktischen Produkthaftung. Für diese sind entsprechende Fehlerkategorien entwickelt worden, wobei bei der Künstlichen Intelligenz der Konstruktionsfehler von besonderer Relevanz ist. Das Verhalten der Künstlichen Intelligenz ist durch ihre deterministische Natur eine Folge ihrer Programmierung und Anlernung und damit Folge ihrer Konstruktion. Ein Konstruktionsfehler liegt vor, »wenn das Produkt schon seiner Konzeption nach unter dem gebotenen Sicherheitsstandard bleibt $\ll{ }^{534}$ Somit ist fraglich, wie der objektive Sorgfaltsmaßstab zur Erkennung und Vermeidung von Konstruktionsfehlern bei Künstlicher Intelligenz zu bestimmen ist. ${ }^{535}$ Es kommt also darauf an, wie der gebotene Sicherheitsstandard zu bemessen ist. Üblicherweise wird bei (verschuldensabhängiger) Produkthaftung ein strenger Sorgfaltsmaßstab angelegt. ${ }^{536}$ Es wird - wie auch in $\S 1$ Abs. 2 Nr. 5 ProdHaftG - auf den Stand der Wissenschaft und Technik abgestellt, ${ }^{537} \mathrm{der}$

530 S. o., Explainable AI Ansätze (S. 65).

531 Ebenso wohl auch Zech, ZfPW 2019, 198, 210.

532 Auch Produzentenhaftung genannt, vgl. Fuchs/Baumgärtner, JuS 2011, 1057.

533 Vgl. Kötz/Wagner, Rn. 606 ff.

534 BGH, U. v. 16.06.2009 - VI ZR 107/08, Rn. 15 - NJW 2009, 2952.

535 Vgl. Etzkorn, MMR 2020, 360, $362 \mathrm{ff}$.

536 Vgl. Rotsch/Hilgendorf, § 10 Rn. 9 f.

537 BGH, U. v. 16.06.2009 - VI ZR 107/08, Ls. 2, NJW 2008, 2952. 
strenger ist als der Stand der Technik. ${ }^{538}$ Sofern veröffentlicht, müssen daher auch Mindermeinungen beachtet werden. ${ }^{539}$ Dies führt unweigerlich zu der Frage, welche Maßstäbe an die Qualitätssicherung eines Herstellers der Künstlichen Intelligenz zu stellen sind, genauer: Ob jeder Fehler, der nicht zum unvorhersehbaren Verhalten gehört, erkannt werden muss.

Allerdings kann nicht erwartet werden, dass ein Hersteller alles Mögliche unternimmt, um Fehler zu entdecken. ${ }^{540}$ Es ist zwar auch bei Künstlicher Intelligenz zumindest theoretisch denkbar, alle möglichen Zustände durchzurechnen, allerdings überschreitet die Rechenzeit bei gegebener Komplexität die typische Lebenserwartung eines Menschen um ein Vielfaches. Deshalb beschränken sich die Sorgfaltspflichten auf das technisch Mögliche und wirtschaftlich Zumutbare. ${ }^{541}$ Im Rahmen dessen können auch technische Normwerke herangezogen werden, sie sind aber nur die untere Grenze dessen, was befolgt werden muss ${ }^{542}$ und stellen zunächst nur eine Vermutung für den Stand der Technik dar. ${ }^{543}$ Bei Künstlicher Intelligenz bestehen derzeit aber noch keine entsprechenden spezifischen Regelungen. ${ }^{544}$ Das ändert allerdings nichts an der Notwendigkeit, im Rahmen des technisch Möglichen und wirtschaftlich Zumutbaren, entsprechende Fehler zu erkennen und zu vermeiden. ${ }^{545}$ Für diese Bestimmung soll sich an den durchschnittlichen Erwartungen des Benutzers orientiert werden. ${ }^{546}$

Deutlich werden die Anforderungen an den Hersteller, das technisch Mögliche und wirtschaftlich Zumutbare zu machen, an den Methoden der Erklärbarkeit von Künstlicher Intelligenz (xAI). Diese sind zwar durchaus Stand der Wissenschaft, aber noch nicht Stand der Technik. ${ }^{547}$ Es dürfte nämlich von einem durchschnittlichen Hersteller von Künstlicher Intelligenz zu erwarten sein, dass er Fehlerklassen erkennen kann, die zumindest mit bekannten und immer mehr etablierten Methoden der Erklärbarkeit von

538 BVerfG, B. v. 08.08.1978 - 2 BvL 8/77, NJW 1979, 359, 362 = BVerfGE 49, 89 - Kalkar I.

539 MünchKommBGB/Wagner, § 1 ProdHaftG Rn. 57

540 BGH, U. v. 16.02.1972 - VI ZR 111/70, VersR 1972, 559; OLG Köln, B. v. 06.04.2006 - 3 U 184/05, NJW 2006, 2272; MünchKommBGB/Wagner, § 823 BGB Rn. 809; Staudinger/Hager, § 823 BGB Rn. F 12.

541 BGH, U. v. 17.10.1989 - VI ZR 258/88, NJW 1990, 906, 908; BGH, U. v. 07.06.1988 - VI ZR 91/87, NJW 1988, 2611, 2614 = BGHZ 104, 323

542 MünchKommBGB/Wagner, § 823 BGB Rn. 815.

543 OLG Celle, U. v. 28.05.2003 - 9 U 7/03, NJW 2003, 2544.

544 Vgl. DIN, Künstliche Intelligenz, S. $1 \mathrm{ff}$.

545 OLG Schleswig, U. v. 19.10.2007 - 17 U 43/07, NJW-RR 2008, 691, 692.

546 BGH, U. v. 07.06.1988 - VI ZR 91/87, NJW 1988, 2611, 2612 = BGHZ 104, 323; OLG Schleswig, U. v. 19.10.2007 - 17 U 43/07, NJW-RR 2008, 691, 692.

547 S. o., Explainable AI Ansätze (S. 65). 
Künstlicher Intelligenz (xAI) gefunden werden können: Der Implementierungsaufwand weicht im Wesentlichen nicht von dem einer Künstlichen Intelligenz ab. Dies gilt zumindest soweit, wie die Nachvollziehbarkeit der Analyse für den Hersteller nicht die Komplexität der xAI-Methode überschreitet. Im Beispielsfall des im vorherigen Kapitel genannten Wolf-Husky-Klassifikators ${ }^{548}$ gehört es wohl im Allgemeinen zu den Sorgfaltspflichten des Herstellers, es zu erkennen und zu vermeiden, dass dieser Klassifikator anstelle von Wölfen und Hunden eigentlich Gras und Schnee unterscheidet. Das gilt umso mehr, wenn dieser Klassifikator nicht mehr isoliert betrachtet wird sondern Teil einer komplexeren Umgebung ist. Ist die Künstliche Intelligenz Bestandteil eines Produktes, von welchem eine erhöhte Gefahr ausgeht, so erwartet der durchschnittliche Benutzer gar einen erhöhten Sicherheitsstandard und damit eine erhöhte Prüfung. Infolgedessen ist nach Art der Künstlichen Intelligenz zu entscheiden, wie weit der Sorgfaltsmaßstab geht. Ein Indiz kann allerdings auch der Kaufpreis sein, da der Benutzer bei höheren Kaufpreisen auch eine bessere Überprüfung erwartet. ${ }^{549}$

Möglicherweise führt aber gerade der beim Hersteller angesetzte objektive Maßstab in gewissen Fällen zu einem Wertungsproblem. Hierfür lässt sich ein einfacher Fall konstruieren: Nimmt man an, ein Hersteller verfügt über eine xAI-Methode, die einen spezifischen Fehler aufzeigen kann, den ein anderer und in sonstiger Hinsicht vergleichbarer Hersteller nicht finden kann, weil er über diese Methode nicht verfügt. Im Weiteren sei angenommen, dass diese Methode vom Hersteller als Know-how geheim gehalten wird und daher nicht dem Stand der Technik entspricht. Sodann stellt dieser Hersteller eine Künstliche Intelligenz her, die über einen durch diese Methode entdeckbaren Fehler verfügt, vergisst aber-also ohne Wollenselement - seine Methode darauf anzuwenden. Bei objektiver Betrachtung läge damit keine Fahrlässigkeit vor, da der Fehler für einen Angehörigen des maßgeblichen Verkehrskreises nicht erkennbar gewesen wäre, subjektiv für den spezifischen Hersteller aber schon. Gewiss ist der Fall nicht spezifisch für Konstellationen mit Künstlicher Intelligenz, denkbar wären auch gleich gelagerte Fälle über Methoden der Materialprüfung. Zur Lösung bietet es sich an, wenn man für diesen Hersteller keinen - sehr 
einsamen - Verkehrskreis annehmen möchte, zumindest subjektive Verschärfungen beim Fahrlässigkeitsmaßstab zuzulassen. ${ }^{550}$

\section{Verschuldensunabhängige Haftung}

Neben der verschuldensabhängigen Haftung bestehen noch die vertragliche Garantiehaftung und die außervertraglichen verschuldensunabhängigen Haftungsansätze. Da keine gesonderte (außervertragliche) Haftung für Künstliche Intelligenz oder autonome Systeme besteht, verbleiben zunächst nur die Haftungsregelungen für besondere technische Phänomene, die Künstliche Intelligenz enthalten können ${ }^{551}$ sowie die Produkthaftung als gesonderte Haftung des Herstellers für Produktschäden, bei Verletzung der in $\S 1$ Abs. 1 S. 1 ProdHaftG genannten Rechtsgüter. ${ }^{552}$ Ohne die Frage der dogmatischen Einordnung der Produkthaftung weiter zu vertiefen, ${ }^{553}$ wird sie im Weiteren als verschuldensunabhängige Haftung angesehen. $\mathrm{Ob}$ Künstliche Intelligenz - als Computerprogramm - eine bewegliche Sache und damit ein Produkt i.S.d. § 2 ProdHaftG ist, ist zwar fraglich, kann aber angenommen werden, auch deshalb, weil der Begriff der beweglichen Sache im Produkthaftungsrecht autonom auszulegen ist. ${ }^{554}$ Im Weiteren deckt sich der Fehlerbegriff des ProdHaftG im Wesentlichen mit dem deliktischen Fehlerbegriff, ${ }^{555}$ der - von Fabrikationsfehlern abgesehen - die deliktischen Sorgfaltspflichten umschreibt. ${ }^{556}$ Daraus folgt, dass unvorhersehbares Verhalten auch im Produkthaftungsrecht keine Haftung auslöst, da es kein Fehler i.S.d. § 3 ProdHaftG ist.

550 BGH, U. v. 10.02.1987 - VI ZR 68/86, Ls. 2, NJW 1987, 1479; BeckOK/Lorenz, $\S 276 \quad$ BGB Rn. 21; Jauernig/Stadler, $\$ 276 \quad$ BGB $\quad$ Rn. 29; MünchKommBGB/Grundmann, $\$ 276$ BGB Rn. 56; so auch MünchKommBGB/Wagner, §823 BGB Rn. 40: "Schließlich wirkt sich die Objektivierung der Fahrlässigkeit niemals zugunsten des Täters aus«, der ansonsten für einen strikten objektiven Fahrlässigkeitsbegriff plädiert, vgl. Kötz/Wagner, Rn. 114 ff.

551 Wie $\S 7$ Abs. 1 StVG oder $\S 25$ AtG.

552 Vgl. Fuchs/Baumgärtner, JuS 2011, 1057, 1061 ff.; Zech, in: Gless/Seelmann, S. 184.

553 Streitig, vgl. MünchKommBGB/Wagner, Einl. ProdHaftG Rn 17 ff.

554 Vgl. MünchKommBGB/Wagner, § 2 ProdHaftG Rn. 17 ff. m.w.N.

555 Entwurf eines Gesetzes über die Haftung für fehlerhafte Produkte, BT-Drs. 11/2447, S. $17 \mathrm{f}$.

556 MünchKommBGB/Wagner, § 3 ProdHaftG Rn 3; zu den einzelnen Produktfehlern vgl. Wagner, AcP 217 (2017), 707, 724 ff. 
Fraglich bleibt insoweit nur, ob jeder Fehler der Künstlichen Intelligenz ein Fehler im Produkthaftungsrecht ist, der eine entsprechende Haftung begründet. Wie auch im Deliktsrecht wird in $\S 1$ Abs. 2 Nr. 5 ProdHaftG die Haftung ausgeschlossen, wenn der Fehler zum Zeitpunkt des Inverkehrbringens nach dem Stand der Wissenschaft und Technik nicht erkannt werden konnte. Insoweit kann hier auf die Ausführungen für die verschuldensabhängige Haftung verwiesen werden. ${ }^{557}$ Auch bei Haftung nach ProdHaftG kann sich bei einer unveröffentlichten und geheim gehaltenen xAI-Methode - die damit nicht zum Stand der Wissenschaft gehört - bei Nichtanwendung nicht auf $\S 1$ Abs. 2 Nr. 5 ProdHaftG berufen werden, da die persönlichen Möglichkeiten der Prüfung immer angewandt werden müssen. ${ }^{558}$

III. Fremdzurechnung des Verhaltens der Künstlichen Intelligenz

Aus den bisherigen Ansätzen folgt, dass für unvorhersehbares Verhalten der Künstlichen Intelligenz keine Haftung für ein beteiligtes Rechtssubjekt begründet werden kann. Möglicherweise tritt Künstliche Intelligenz, zumindest aber autonome Systeme, wie ein Dritter in Erscheinung. Im Gegensatz zu anderen technischen Einrichtungen wird daher diskutiert, ob nicht das Verhalten der Künstlichen Intelligenz einem beteiligten Rechtssubjekt zugerechnet werden kann, wie auch in den Situationen, in welchen das Verhalten eines Dritten zugerechnet wird.

\section{Künstliche Intelligenz als Erfüllungsgehilfe}

Ein diskutierter Ansatz ist das Einstehenmüssen für das Verhalten einer technischen Einrichtung - im Besonderen einer Künstlichen Intelligenz ${ }^{559}$ - durch analoge Anwendung des $\S 278$ BGB zu erreichen. ${ }^{560}$ Hier wird das eigene Verschulden einer natürlichen Person als Maßstab genommen. Problematisch ist aber, dass keine vergleichbare Situation ${ }^{561}$ bzw. nichts

557 S. o., Fahrlässigkeit (S. 120).

558 Vgl. BGH, U. v. 09.01.1990 - VI ZR 103/89, NJW-RR 1990, 406.

559 Hacker, RW 2018, 243, 250 ff.; Keßler, MMR 2017, 589, 593; Schirmer, JZ 2016, 660, 665; Teubner, AcP 218 (2018), 155, 185 ff.

560 Wolf, JuS 1989, 899, 901 f.; Wulf/Burgenmeister, CR 2015, 404, 407.

561 Vgl. Rüthers/Fischer/Birk, Rn. 889. 
Gleichartiges $^{562}$ vorliegt. Zwar wird auf den Sorgfaltsmaßstab des Schuldners und nicht auf den des Erfüllungsgehilfen abgestellt, ${ }^{563}$ dennoch ist dem Wortlaut nach ein Verschulden des Erfüllungsgehilfen notwendig. Zwar wird vertreten, dass eine Haftung des Schuldners für Handlungen von verschuldensunfähigen Erfüllungsgehilfen eintreten kann, ${ }^{564}$ dies ist aber mit dem Verweis des $\S 276$ Abs. 1 S. 2 BGB auf $\S \S 827,828$ BGB nicht vereinbar. ${ }^{565}$ Zudem kann auch bei einem verschuldensunfähigen, aber menschlichen Erfüllungsgehilfen von nicht-deterministischem Verhalten ausgegangen werden. Wie schon beim eigenen Verschulden gezeigt, kann eine Künstliche Intelligenz aber aufgrund der deterministischen Eigenschaft kein Verschulden treffen. ${ }^{566}$ Klingbeil schlägt deshalb vor, durch Schaffung eines neuen $\S 279$ BGB das Verhalten einer Maschine - und damit wohl auch einer Künstlichen Intelligenz - dem Schuldner zuzurechnen, wenn in einem gleich gelagerten Fall mit einem Erfüllungsgehilfen eine Haftung begründet werden könnte, ohne dass es auf ein Verschulden der Künstlichen Intelligenz ankommt. ${ }^{567}$ Allerdings weist die Lösung eine indirekte Verschuldensprüfung auf. Es wäre weiterhin zu prüfen, ob in einer gleich gelagerten Situation bei einem Erfüllungsgehilfen eine entsprechende Haftung vorliegen würde. Damit wird bei der Künstlichen Intelligenz eine Art Verschulden fingiert.

\section{Künstliche Intelligenz als Verrichtungsgehilfe}

Eine weitere Möglichkeit ${ }^{568}$ besteht darin, Künstliche Intelligenz als Verrichtungsgehilfen $\mathrm{zu}$ betrachten und somit $\S 831$ BGB analog anzuwenden. ${ }^{569}$ Da es auf das Verschulden des Verrichtungsgehilfen selbst

562 Vgl. Larenz/Canaris, Methodenlehre, S. 202 ff.

563 BGH, U. v. 15.12.1959 - VI ZR 222/58, NJW 1960, 669, 671 = BGHZ 31, 358.

564 MünchKommBGB/Grundmann, § 278 BGB Rn. 50; Larenz, Lehrbuch des Schuldrechts I, S. $302 \mathrm{f}$.

565 Staudinger/Caspers, § 278 BGB Rn. 68.

$566 \mathrm{Im}$ Ergebnis ebenso BeckOGK/Schaub, §278 BGB Rn. 17; MünchKommBGB/Grundmann, § 278 BGB Rn. 46; Staudinger/Caspers, § 278 BGB Rn. 5; Foerster, ZfPW 2019, 418, 431; Klingbeil, JZ 2019, 718, 719 ff.; Schaub, JZ 2017, 342, 343; Müller-Hengstenberg/Kirn, MMR 2014, 307, 310 f.; Grapentin, S. 131; Reusch/Weidner, Rn. 53 ff.

567 Klingbeil, JZ 2019, 718, $723 \mathrm{f}$.

568 Die unter strenger Betrachtung keine Fremdzurechnung ist.

569 Keßler, MMR 2017, 589, 593 f.; Denga, CR 2018, 69, 74 ff.; bejahend auch Zech, ZfPW 2019, 198, 211. 
nicht ankommt, ${ }^{570}$ sondern nur auf das Verschulden des Geschäftsherren bei Auswahl und Leitung des Verrichtungsgehilfen, ist die Frage nach dem Verschulden der Künstlichen Intelligenz selbst nicht von Relevanz. ${ }^{571}$ Für eine analoge Anwendung muss aber eine planwidrige Regelungslücke und eine vergleichbare Interessenlage bestehen. Bejaht man die planwidrige Regelungslücke, bleibt dennoch unklar, ob eine vergleichbare Interessenlage besteht. Dagegen könnte nach wie vor die deterministische Eigenschaft einer Künstlichen Intelligenz sprechen. Ursprünglich waren nur Menschen - und damit nicht-deterministische Entitäten - in Betracht gezogen worden, insbesondere Angestellte und Arbeiter. ${ }^{572}$ Die Übernahme menschlicher Handlungen durch Künstliche Intelligenz könnte für eine gleiche Interessenlage sprechen. Eine solche Analogie würde aber jegliche Art von (softwaregesteuerter) Entität umfassen, deren Tätigkeit einem Verrichtungsgehilfen nahekommt. Ferner muss auch hier ein Verschulden auf Seiten der Geschäftsherren vorliegen, was weiterhin begründet werden muss.

\section{Kausalität}

Allgemein wird unter Kausalität verstanden, dass ein Ereignis für eine Folge ursächlich ist. ${ }^{573} \mathrm{Im}$ Haftungsrecht muss somit ein ursächlicher $\mathrm{Zu}$ sammenhang zwischen einem schädigenden Ereignis (Pflichtverletzung) und einem Schaden bestehen. ${ }^{574}$ Bezogen auf die spezifischen Fälle der Künstlichen Intelligenz muss also ein kausaler Zurechnungszusammenhang zwischen einem Schaden und einem KI-bezogenen Ereignis existieren. Da dieses KI-bezogene Ereignis eine Pflichtverletzung sein muss, verbleiben bei Annahme fehlender Rechtssubjekteigenschaft der Künstlichen Intelligenz - nur Pflichtverletzungen der Beteiligten. ${ }^{575}$

Bei der Kausalität wird zwischen der haftungsbegründenden Kausalität und der haftungsausfüllenden Kausalität unterschieden. ${ }^{576}$ Die haftungsbegründende Kausalität ist der Zurechnungszusammenhang zwischen dem

570 Vgl. BGH, U. v. 15.10.2013 - VI ZR 124/12, Rn. 11, NJW 2014, 1380.

571 Denga, CR 2018, 69, 75.

572 Vgl. Mugdan II, $411 \mathrm{f}$.

573 Vgl. Musielak, JA 2013, 241.

574 Vgl. BGH, U. v. 01.10.1987 - IX ZR 117/86, NJW 1988, 200, 202.

575 Vgl. Zech, ZfPW 2019, 198, 206 ff.

576 Was streng genommen erst bei der Einschränkung der Kausalität von Bedeutung ist, vgl. MünchKommBGB/Oetker, § 249 BGB Rn. 105. 
schädigenden Ereignis und der Rechtsgutsverletzung, die haftungsausfüllende Kausalität der Zurechnungszusammenhang zwischen der Rechtsgutverletzung und den entstandenen Schäden. ${ }^{577}$ Diese Unterscheidung ist wichtig, weil bei Künstlicher Intelligenz gerade die haftungsbegründende Kausalität die entsprechenden Probleme aufweist. Hat das Verhalten einer Künstlichen Intelligenz eine Rechtsgutsverletzung zur Folge, bleibt fraglich, ob hierin eine kausale Pflichtverletzung eines Beteiligten zu erblicken ist.

\section{1. Äquivalenztheorie}

Der Äquivalenztheorie nach ist jedes Ereignis kausal, welches nicht hinweggedacht werden kann, damit das (schädigende) Ereignis derart oder zumindest zu der Zeit eingetreten ist (conditio sine qua non) ${ }^{578}$ Für den Eintritt des damit begründeten Kausalverlaufs dürfen weitere (hypothetische) Umstände zudem nicht hinzugedacht werden. ${ }^{579}$ Nicht äquivalent kausal sind damit die Ereignisse, die für das Entstehen des Schadens keine Rolle spielen. ${ }^{580}$ Die Bedingung der Äquivalenztheorie ist allerdings nur notwendige, aber keinesfalls hinreichende Bedingung für eine Kausalität. ${ }^{581}$ Resultiert aus dem Verhalten von Künstlicher Intelligenz ein Schaden, so folgt daraus, dass sowohl die Herstellung als auch der Betrieb der Künstlichen Intelligenz äquivalent kausal für die Rechtsgutsverletzung und damit auch für den Schaden sind, wenn das schädigende Ereignis schon ohne die bloße Existenz der Künstlichen Intelligenz nicht eingetreten wäre. ${ }^{582}$ Die Künstliche Intelligenz kann also im Hinblick auf den eingetretenen Erfolg nicht hinweggedacht werden. Das spezifische Verhalten oder die inneren Zustände spielen hier keine Rolle, sodass hier keine KI-spezifischen Besonderheiten bestehen. Im Umkehrschluss heißt dies: Wenn der gleiche Schaden ohne die Künstliche Intelligenz eingetreten wäre, kann schon nach der Äquivalenztheorie keine Haftung für die Herstellung oder den Betrieb der Künstlichen Intelligenz begründet werden. Da die Äquivalenztheorie

577 Vgl. Larenz, Lehrbuch des Schuldrechts I, S. 432.

578 MünchKommBGB/Oetker, § 249 BGB Rn. 103.

579 BGH, U. v. 06.06.2013 - IX ZR 204/12, Rn. 20, NJW 2013, 2345; BGH, U. v. 05.05.2011 - IX ZR 144/10, Rn. 35, NJW 2011, $2960=$ BGHZ 189, 299.

580 Vgl. BGH, U. v. 11.05.1951 - I ZR 106/50, NJW 1951, $711=$ BGHZ 2, 138.

581 MünchKommBGB/Oetker, § 249 BGB Rn. 104; Staudinger/Schiemann, § 249 BGB Rn. 8.

582 Hier freilich noch ohne Trennung von haftungsbegründender und haftungsausfüllender Kausalität. 
allerdings dennoch zu einer weiten Haftung führen kann, wird sie im Weiteren durch die Adäquanztheorie und den Schutzzweck der Norm eingeschränkt. $^{583}$

\section{Adäquanztheorie}

Zweck der Adäquanztheorie ist es, unwahrscheinliche Kausalverläufe auszuschließen, d.h. solche »außerhalb jeder Lebenserfahrung $\ll .{ }^{584}$ Es sind also nur solche Ereignisse adäquat, bei welchen »eine Tatsache im Allgemeinen und nicht nur unter besonders eigenartigen, ganz unwahrscheinlichen und nach dem regelmäßigen Verlauf der Dinge außer Betracht zu lassenden Umständen zur Herbeiführung eines Erfolges geeignet war ${ }^{585} \mathrm{Zu}$ deren Beurteilung wird auf einen Beobachter mit dem Wissensstand zum Zeitpunkt des schädigenden Ereignisses abgestellt. ${ }^{586}$ Für ein Verhalten der Künstlichen Intelligenz heißt dies, dass für beteiligte Rechtssubjekte in bestimmten Situationen keine adäquate Zurechnung möglich ist. Folge der im vorherigen Kapitel herausgearbeiteten Unterscheidung zwischen Fehler und unvorhersehbarem Verhalten ${ }^{587}$ ist, dass bei Letzterem nie eine adäquate Kausalität bejaht werden kann, da es immer außerhalb des Wissenstandes des Beobachters liegt. Somit würde unvorhersehbares Verhalten der Künstlichen Intelligenz bei Anwendung der Adäquanztheorie per se keine Haftung begründen. Selbst ein optimaler Beobachter hätte das unvorhersehbare Verhalten nicht vorhersehen können, auch wenn das Verhalten Folge der deterministischen Eigenschaft der Künstlichen Intelligenz ist. Somit ist ein verschuldensabhängiger Haftungsansatz bei Künstlicher Intelligenz nicht nur auf der Ebene des Verschuldens mit Einschränkungen verbunden, sondern auch - bei Anwendung der Adäquanztheorie im Rahmen der Haftungsbegründung - auf der Ebene der Kausalität. ${ }^{58}$

Daraus folgt, dass gerade die Haftung für Schäden aus bedeutsamen, aber aus ex ante Sicht nicht wahrscheinlichen Ereignissen, bei Anwendung der

583 BGH, U. v. 10.07.2012 - VI ZR 127/11, Rn. 12, NJW 2012, 2964; BGH, U. v. 11.11.1999 - III ZR 98/99, NJW 2000, 947, 948; vgl. Spindler, AcP 208 (2008), 283, $285 \mathrm{ff}$.

584 BGH, U. v. 07.03.2001 - X ZR 160/99, NJW-RR 2001, 887, 888.

585 BGH, U. v. 23.10.1951 - I ZR 31/51, VersR 1952, $128=$ BGHZ 3, 261, 266 f.

586 Vgl. BGH, U. v. 16.04.2002 - VI ZR 227/01, NJW 2002, 2232, 2233; kritisch Palandt/Grüneberg, Vorb v § 249 BGB Rn. 28; Staudinger/Schiemann, § 249 BGB Rn. 17 ff.; Larenz, Lehrbuch des Schuldrechts I, S. 436 ff.

587 S. o., Vom Sein und Sollen (S. 64).

588 Was auch bei einer analogen Anwendung der $\S$ 278, 831 BGB der Fall wäre. 
Adäquanztheorie außen vor wäre. Dieses Problem betrifft auch die Ansätze der Fremdzurechnung des Verhaltens der Künstlichen Intelligenz, also die analoge Anwendung des $\S 831$ BGB oder die Schaffung eines neuen $\S 279$ BGB. Auch hier könnte nur für Fehler, nicht aber für unvorhersehbares Verhalten gehaftet werden. Da die Adäquanztheorie aber an das (menschliche) Verhalten des Schädigers anknüpft, ${ }^{589}$ ist sie zumindest bei der Gefährdungshaftung ${ }^{590}$ und bei der Produkthaftung ${ }^{591}$ im Rahmen der Haftungsbegründung nicht anwendbar. ${ }^{592}$ Dort geht es - unabhängig vom (menschlichen) Verhalten der Beteiligten - um eine reine Risikozurechnung. ${ }^{593}$

\section{Schutzzweck der Norm}

Durch den Schutzzweck der Norm kann die kausale Zurechnung jedoch erweitert oder begrenzt werden. Hierbei soll durch teleologische Auslegung der jeweiligen Norm ermittelt werden, ob in Folge des Zwecks der Norm eine Haftung für das Ereignis eintreten soll. ${ }^{594}$ Insbesondere bei spezialgesetzlicher, verschuldensunabhängiger Haftung kann hierdurch überprüft werden, für welche Ereignisse gehaftet werden soll. Beispielsweise liegt bei der Produkthaftung der Schutzzweck darin, den Verkehr vor gefährlichen Fehlern im Produkt zu schützen. ${ }^{595}$ Allerdings sind Alltagsgefahren aus dem Schutzzweck der Norm in der Regel ausgenommen. ${ }^{596}$ Allgemein soll daher bei einer Gefährdungshaftung auch nach dem Schutzzweck der Norm nur für kausale Folgen aus der Gefahr gehaftet werden. ${ }^{597}$

589 Vgl. BGH, U. v. 21.11.2017 - X ZR 30/15, Rn. 23, NJW 2018, 861.

590 BGH, U. v. 13.07.1982 - VI ZR 113/81, NJW 1982, 2669; BGH, U. v. 27.01.1981 - VI ZR 204/79, NJW 1981, 983 = BGHZ 79, 259.

591 BeckOK/Förster, § 1 ProdHaftG Rn. 30; MünchKommBGB/Wagner, § 1 ProdHaftG Rn. 20, wobei dort aber an einer Verhaltenspflicht angeknüpft wird, vgl. Wagner, AcP 217 (2017), 707, 711 f.; Zech, ZfPW 2019, 198, 213.

592 Kritisch zum Deliktsrecht auch Kötz/Wagner, Rn. 193.

593 Streng genommen handelt es sich somit um das Risiko, das sich im Verhalten der Entität verwirklichen kann, von der eine Gefährdung ausgeht. Dieses ist allerdings kein menschliches Verhalten.

594 Spezieller bei BeckOK/Flume, § 249 BGB Rn. 288; Medicus/Lorenz, Rn. 682.

595 BeckOK/Förster, § 1 ProdHaftG Rn. 30.

596 BGH, U. v. 20.05.2014 - VI ZR 381/13, Rn. 10 NJW 2014, $2190=$ BGHZ 201, 263; Lange/Schiemann, S. $145 \mathrm{ff}$.

597 BGH, U. v. 26.02.2013 - VI ZR 116/12, Rn. 12, NJW 2013, 1679. 


\section{Folgen}

Im Vergleich zu bisherigen technischen Phänomenen zeigt sich, dass auf der Ebene des Verschuldens und auf der Ebene der Kausalität Haftungslücken entstehen können, ${ }^{598}$ deren Grundlage bei beiden Aspekten das unvorhersehbare Verhalten einer Künstlichen Intelligenz ist. Dies gilt gleichermaßen für das Vertrags- und das Deliktsrecht. Für den Fall, dass sich das Verhalten einer Künstlichen Intelligenz aber einem Haftungssubjekt zurechnen lässt, gleicht die Situation der Haftung der Beteiligten bei herkömmlichen technischen Phänomenen. Aus der Begründung der aufgezeigten Haftungslücken folgt zugleich, dass die Probleme auch bestehen, wenn eine Künstliche Intelligenz oder eine bezogene Entität das Haftungssubjekt wäre; eine Künstliche Intelligenz kann a priori kein Verschulden treffen und das unvorhersehbare Verhalten ist nicht adäquat kausal. Allein aus diesem Grund bringt auch eine (Teil-)Rechtsfähigkeit im Haftungsrecht keinen Mehrwert; eine (Teil-)Rechtsfähigkeit ändert nur das Vermögen, das zur Haftung herangezogen wird: Das einer (teil-)rechtsfähigen Künstlichen Intelligenz oder das eines der beteiligten Akteure. Das gleiche Ergebnis entsteht bei fremdzurechnenden Haftungsansätzen de lege ferenda, sei es durch eine analoge Anwendung des $\S 831$ BGB oder durch Schaffung eines neuen $\S 279$ BGB. Diese würden zwar die Verschuldensproblematik überwinden, nicht aber den nicht vorhandenen, adäquaten haftungsbegründenden Kausalverlauf.

Aus dem bisher Festgestellten folgt, dass nur die verschuldensunabhängigen Haftungsansätze für die bestehenden Haftungslücken eine Lösung versprechen. ${ }^{599}$ Eine spezifische verschuldensunabhängige Haftung für das Verhalten von Künstlicher Intelligenz besteht bisher nicht. Betrachtet man die bestehenden verschuldensunabhängigen Haftungsregelungen, so greift die Produkthaftung nur für den Hersteller einer Künstlichen Intelligenz und zudem nur für bestimmte Rechtsgüter. Auch die bestehenden Gefährdungshaftungen knüpfen grundsätzlich an andere Gefahrenquellen an. ${ }^{600}$ Bestünde eine Gefährdungshaftung für das (unvorhersehbare) Verhalten von Künstlichen Intelligenzen, so würde gerade sie den Zweck verfolgen, für die unwahrscheinlichen und deshalb schwer oder

598 Vgl. zum Deliktsrecht Teubner, AcP 218 (2018), 155, 190 ff.

599 Sofern man am Merkmal der Adäquanztheorie bei der Haftungsbegründung festhält.

600 Für Kraftfahrzeuge und autonomes Fahren vgl. Borges, CR 2016, 272. 
nicht voraussehbaren Ereignisse eine Haftungsgrundlage zu schaffen. Die bestehenden Zurechnungsprobleme würden dann wegfallen. ${ }^{601}$

$\mathrm{Zu}$ diskutieren bleibt, ob es sich bei Betrieb oder Herstellung einer Künstlichen Intelligenz wirklich um die Schaffung oder Unterhaltung einer Gefahr handelt, da eine Gefährdungshaftung eine verschuldens- und rechtswidrigkeitsunabhängige Haftung für Schäden einer aus der Betätigung einhergehenden Gefahr wäre. ${ }^{602} \mathrm{Ob}$ das bei jeder Künstlichen Intelligenz der Fall ist, ist fraglich, da grundsätzlich nicht jede Künstliche Intelligenz per se gefährlich ist. Beispielsweise ist das Risiko, das durch die Herstellung oder mit dem Betrieb eines (selbstlernenden) Schachcomputers einhergeht, durchaus überschaubar, wenn nicht gar inexistent. ${ }^{603}$

Nicht überzeugen kann das Argument, welches gegen eine Gefährdungshaftung hervorgebracht wird, dass das Verhalten einer Künstlichen Intelligenz unvorhersehbar sei und damit auch grundsätzlich für unvorhersehbare Schäden zu haften sei. ${ }^{604}$ Betrachtet man bestehende Gefährdungshaftungen, wie die für den Betrieb von Kernkraftwerken aus $\S 25 \mathrm{AtG}$, so sind die Schäden und Risiken, die damit einhergehen können, ebenfalls unvorhersehbar. Dennoch darf die mögliche Innovationshemmung, die mit einer weitgehenden Gefährdungshaftung verbunden sein kann, nicht übersehen werden. Ob und wie eine spezifische Gefährdungshaftung weiter auszugestalten ist, verbleibt daher der weiteren Diskussion. ${ }^{605}$

\section{E. Wissenszurechnung}

Zuletzt besteht noch die Zurechnung des Wissens, welche möglicherweise auch haftungsrelevant ist. Bei Künstlicher Intelligenz ist diese Zurechnung aber aufgrund deren Wesen als Computerprogramm wenig komplex. ${ }^{606} \mathrm{Die}$ Wissenszurechnung wurde bisher vor allem dann diskutiert, wenn es ein personales Auseinanderfallen von Handelndem und Wissendem gibt. ${ }^{607}$ In der Regel erfolgt die Zurechnung des Wissens von natürlichen Personen oder Informations- bzw. Wissensspeichern zu einer (weiteren) Person. ${ }^{608}$

601 Im Ergebnis ebenso Spindler, CR 2015, 766, 775.

602 Brox/Walker, Bes. SchuldR., § 54 Rn. 2; Wandt, § 22 Rn. 1.

603 Von möglichen Suchtgefahren einmal abgesehen.

604 Denga, CR 2018, 69, 76.

605 Vgl. Wagner, in: Faust/Schäfer, S. 6 ff.; Teubner, AcP 218 (2018), 155, 191 ff.; Zech, in: Gless/Seelmann, S. 195 f.; Zech, ZfPW 2019, 198, 209 ff.

606 Anders Hacker, RW 2018, 243, 267 ff.

607 Vgl. Grigoleit, ZHR 181 (2017), 160, 166 f.

608 S. Fn. 269. 
Was in diesem Sinne als »Wissen« zu verstehen ist, bleibt oft vage. Eine allgemeine Definition fehlt. ${ }^{609}$ Gelegentlich findet sich eine Gleichsetzung mit dem Begriff »Kenntnis«, ohne diese näher zu konkretisieren. ${ }^{610} \mathrm{Nach}$ Klingmüller ist Wissen »ganz allgemein die Summe von Erkenntnissen, das heißt von Geschehnissen, feststehenden Fakten, aber auch von der natürlichen allgemeinen Ordnung $« .{ }^{611}$ Hacker hat die Frage der Zurechnung auf die entstehenden Probleme bei Künstlicher Intelligenz übertragen. In diesem Fall geht es dann um die Zurechnung vom Wissen der Künstlichen Intelligenz auf ein Rechtssubjekt. ${ }^{612}$ Neben der dogmatischen Kritik an der Wissenszurechnung ${ }^{613}$ und der Frage, ob sie bei einer strikten Verhaltenszurechnung überhaupt noch Relevanz hat, ${ }^{614}$ ist die Frage zu berücksichtigen, ob die Art des Wissens einer Künstlichen Intelligenz mit der Art des Wissens eines Menschen übereinstimmt. Wie gezeigt, kann eine Künstliche Intelligenz kein implizites Wissen haben, ${ }^{615}$ sodass sich das (explizite) Wissen einer Künstlichen Intelligenz nicht vom Wissen herkömmlicher Computer bzw. dem Aktenwissen unterscheidet. Daher bestehen hier keine KI-spezifischen Probleme, die über bisherige technische Phänomene als Wissensspeicher hinausgehen. ${ }^{616}$

609 Vgl. Wagner, ZHR 181 (2017), 203 ff.; Hacker, RW 2018, 243, 267.

610 Vgl. Köhler/Bornkamm/Feddersen/Köhler, § 11 UWG Rn. 1.27.

611 Klingmüller, in: Möglichkeiten der Wissenszurechnung, S. 2.

612 Hacker, RW 2018, 243, $267 \mathrm{ff}$.

613 Grigoleit, ZHR 181 (2017), 160 ff.; rechtsvergleichend auch Wagner, ZHR 181 (2017), $203 \mathrm{ff}$.

614 Wagner, ZHR 181 (2017), 203, 270 ff.

615 S. o., Wissen und dessen Repräsentation (S. 66); so aber Hacker, RW 2018, 243, $271 \mathrm{f}$., der allerdings hilfsweise von Nichtwissen ausgeht.

616 Zum Wissen von Computern in der Wissenszurechnung vgl. Medicus, in: Möglichkeiten der Wissenszurechnung, S. 7 ff; für explizites Wissen von Künstlicher Intelligenz Hacker, RW 2018, 243, 273 ff. 


\section{$\S 4$ Zusammenfassung und Thesen}

Das vermehrte Aufkommen von Künstlicher Intelligenz führt dazu, dass auch die rechtswissenschaftliche Debatte um ein weiteres technisches Phänomen reicher geworden ist. Wie bei jedem technischen Phänomen, ist auch für eine rechtswissenschaftliche Untersuchung ein gewisser Grad an technischer Kenntnis des Phänomens unerlässlich, vor allem dann, wenn das Phänomen schwer greifbar und kompliziert ist. Diese Untersuchung hat die notwendigen Grundlagen mit ihrem notwendigen technischen Gehalt dargestellt und mit den damit verbundenen Auswirkungen auf die Rechtsgeschäftslehre und das Haftungsrecht zwei grundlegende Felder des allgemeinen Zivilrechts bearbeitet. Im Einzelnen:

1. Die Übersetzung des englischsprachigen Begriffs »artificial intelligence« zu »Künstlicher Intelligenz« fördert bereits ein begriffliches Fehlverständnis, in das Phänomen der Künstlichen Intelligenz einen künstlichen Intellekt hineinzuinterpretieren und somit einen fehlgeleiteten Vergleich von Maschinen bzw. einer Künstlichen Intelligenz mit dem Menschen zu versuchen. Schon vom Begriffsursprung her sind die Begriffe »intelligence « und »Intelligenz« nicht deckungsgleich und haben sich auch in der deutschen und englischen Sprache unterschiedlich fortentwickelt. Gerade der englische Begriff »intelligence« ist weiter als der deutsche Begriff »Intelligenz«; es schwingt keine intellektuelle Wertung mit. Betrachtet man zudem den vorgeschlagenen Alternativbegriff »computional rationality«, zeigt sich, dass eine solche Wertung auch bei der Begriffswahl nicht gewollt war. Folglich kann der Begriff »Künstliche Intelligenz« als wertfreier Eigenname zur Beschreibung bestimmter technischer Entitäten angesehen werden. $^{617}$

2. Die juristische Betrachtung muss die technischen Eigenarten des Phänomens zwingend beachten. Also sind nur jene Phänomene zu untersuchen, die technisch möglich sind bzw. deren technische Umsetzung zumindest wahrscheinlich erscheint: Aus diesem Grund fällt das (philosophische) Gedankenspiel der sog. »starken Künstlichen Intelligenz« aus der Betrachtung heraus. Zudem sind die weiteren Grenzen der algorithmischen Vorgehensweise und der möglichen Berechenbarkeit von Problemen zu beachten. Somit muss mit Vergleichen zum Menschen äußerst vorsichtig 
agiert werden. Bei einer Künstlichen Intelligenz kann nur das Verhalten bzw. ihr Handeln beobachtet werden. Es besteht weder ein »künstlicher Wille« noch ein Prozess des Denkens in dem Sinne, wie ein Mensch denkt. ${ }^{618}$

3. Eine umfassend anerkannte technische Definition für Künstliche Intelligenz fehlt. Gleichwohl kann Künstliche Intelligenz als ein Computerprogramm zur verbesserten Lösung von bestimmten Problemen verstanden werden, das aufgrund immer leistungsfähigerer Computer und wachsender Datenmengen steigende Fähigkeiten erlangt und somit auch menschliche Handlungen übernehmen kann, was freilich eine oberflächliche Betrachtung ist. Daraus folgt auch, dass etwas, das früher einmal als Künstliche Intelligenz angesehen wurde, heute gängige Informatik sein kann; bestes Beispiel ist der Schachcomputer. ${ }^{619}$

4. Bei näherer technischer Betrachtung zeigt sich, dass unter dem Begriff Künstliche Intelligenz nicht ein generischer Ansatz zu verstehen ist und es daher »die« Künstliche Intelligenz nicht gibt. Es gibt vielmehr eine Vielzahl von Methoden der Künstlichen Intelligenz, wie Suchstrategien, Entscheidungsbäume, Clustering-Algorithmen, Fuzzylogiken, Semantic Web Systeme oder Evolutionäre Algorithmen. Manche KI-Methoden können zur Lösung des gleichen Problems herangezogen werden, während sich andere KI-Methoden fundamental von diesen unterscheiden und somit für gänzlich andere Arten von Problemen gedacht sind. Daher ist Künstliche Intelligenz zunächst nur ein Oberbegriff für viele verschiedene Methoden. Zudem ist nur ein Teil dieser KI-Methoden selbstlernend - also zum »Machine Learning « gehörend - sodass das Merkmal »selbstlernend « keine Voraussetzung für eine Künstliche Intelligenz ist. Gänzlich zu unterscheiden von Künstlicher Intelligenz sind »Big Data«-Methoden, welche zwar zusammen mit Künstlicher Intelligenz verwendet werden können, allerdings ein ganz anderes Konzept sind. ${ }^{620}$

5. Die Eigenschaften von Künstlicher Intelligenz lassen sich am besten an der weit verbreiteten Methode des (Künstlichen) neuronalen Netzes illustrieren. Diese Methode besteht aus einer Menge von Input-, Zwischenund Outputneuronen, die zu Beginn mit zufälligen Werten belegt werden. ${ }^{621}$ Im Rahmen des sog. »Trainings « werden diese Werte dann hin zum eigentlichen Zweck optimiert. ${ }^{622}$ In der späteren Anwendung bleiben die im

618 S. o., Problematik des Vergleichs zum Menschen (S. 26).

619 S. o., Definitionsversuche (S. 30).

620 S. o., Technologien der Künstlichen Intelligenz (S. 32).

621 S. o., Modelldarstellung (S. 45).

622 S. o., Trainingsphase (S. 47). 
Training ermittelten Gewichte unverändert. ${ }^{623}$ Zwar gibt es auch hier weiterlernende Systeme, bei diesen werden aber Trainings- und Anwendungsphase abwechselnd ausgeführt oder das Training läuft im Hintergrund mit einem anderen neuronalen Netz ab und dessen Ergebnisse werden regelmäßig in das neuronale Netz übernommen, welches die eigentliche Anwendung darstellt. ${ }^{624}$

6. Durch die abstrakte Betrachtung von KI-Methoden werden zwar gewisse Eigenschaften dieser Methoden deutlich, insbesondere welche Problemklassen sie zu lösen vermögen. Allerdings zeigt diese Art der Betrachtung nicht, wie eine reale Künstliche Intelligenz auf ihre Umgebung, also die reale Welt wirkt, vor allem nicht, welche Eigenarten ihr Verhalten begründet und welchen Grenzen es unterliegt. Eine Künstliche Intelligenz wirkt nur auf die Umgebung, wenn eine oder mehrere KI-Methoden für ein bestimmtes Problem (real) implementiert werden. Dies geschieht über die Implementierung des Konzepts in ein reales Computerprogramm. ${ }^{625}$ Damit handelt es sich bei Künstlicher Intelligenz um (klassische) Computersoftware bzw. -programme und sie unterliegt damit auch deren Restriktionen. Computerprogramme sind eine konkrete Umsetzung von einem oder mehreren Algorithmen. Ein Algorithmus im Sinne der Informatik ist eine (wohldefinierte) Vorschrift, welche aus einer Eingabe eine Ausgabe erzeugt. ${ }^{626}$ Eine Eigenschaft eines Algorithmus ist, dass er bei gleicher Eingabe immer das gleiche Ergebnis ausgibt. Sofern ein Algorithmus zu einem Ende kommt (terminiert), folgt daraus, dass Algorithmen sowohl aus philosophischer als auch aus informatischer Sicht deterministisch sind. ${ }^{627}$ Dies hat die Konsequenz, dass auch (real implementierte) Künstliche Intelligenz immer deterministisch ist. Zwar bestehen KI-Methoden, die unter Ausnutzung des Zufalls funktionieren, allerdings ist dieser Zufall in Computersystemen entweder eine zufällige Eingabe oder sog. »Pseudozufall«. Daher ändert auch die Ausnutzung des Zufalls nichts an der deterministischen Eigenschaft einer Künstlichen Intelligenz. ${ }^{628}$

7. Aus der Komplexität, die eine implementierte Künstliche Intelligenz erreicht, folgt, dass nicht alle möglichen Eingaben und alle möglichen $\mathrm{Zu}-$ stände vorab berechnet und überprüft werden können. Es kann sich somit

623 S. o., Anwendungsphase (S. 49).

624 S. o., Sonderfall: Weiterlernende Systeme (S. 49).

625 S. o., Vom Modell über den Algorithmus zur Computersoftware (S. 52).

626 S. o., Allgemeiner Algorithmus-Begriff (S. 53).

627 S. o., Determinismus (S. 58).

628 S. o., Randomisierte Algorithmen und Pseudozufall (S. 54) und Konvergierender bzw. stochastischer Determinismus (S. 60). 
bei der Evaluation der Künstlichen Intelligenz nur auf die Eingaben beschränkt werden, die als wahrscheinlich gelten. Das gilt freilich auch für andere komplexe Software, erreicht aber durch die fortschreitende Entwicklung auf dem Gebiet der Künstlichen Intelligenz eine neue Ebene. Es können daher nur noch statistische Aussagen über das Verhalten von Künstlicher Intelligenz getroffen werden. Deshalb kann eine Künstliche Intelligenz ein Verhalten zeigen, das nicht demjenigen entspricht, das geplant war. Ist ein solches Verhalten im Vorhinein anhand getesteter Eingaben oder mit weiteren Methoden, die dem Stand der Wissenschaft und Technik entsprechen, erkennbar, so handelt es sich um einen Fehler der Künstlichen Intelligenz. Verhalten, das außerhalb dieser Kriterien liegt, ist unvorhersehbares Verhalten. Dennoch sind beide Arten dieses Verhaltens streng deterministisch; ein freier Wille der Maschine wird nicht begründet. ${ }^{629}$ Eine Gruppe von besonders relevanten weiteren Methoden zur Erkennung von Fehlern sind die verschiedenen Methoden der Explainable AI (xAI). Diese - im Vergleich zur Implementierung einer Künstlichen Intelligenz - ohne weitere Schwierigkeiten implementierbaren Methoden erlauben es, Aussagen über das Verhalten einer Künstlichen Intelligenz zu treffen, die über einen reinen Test möglicher Eingaben hinausgehen, und damit weitere Fehler zu erkennen. ${ }^{630}$

8. Neben dem Verhalten und Willen einer Künstlichen Intelligenz verbleibt die Frage nach dem Wissen der Künstlichen Intelligenz. Zwar verfügt eine Künstliche Intelligenz über Wissen, dies darf aber nicht überbewertet werden. Im Sinne der Informatik stellt Wissen nur eine Vernetzung von Informationen dar. Die Unterteilung aus dem Wissensmanagement in »implizites Wissen« und »explizites Wissen« spielt keine Rolle. Eine Künstliche Intelligenz kann nicht über implizites Wissen verfügen, vielmehr gleicht das mögliche Wissen einer Künstlichen Intelligenz dem möglichen Wissen jedes anderen Computers. ${ }^{631}$

9. Resultierend aus den betrachteten Attributen des Verhaltens, des Willens und des Wissens, verbleibt die juristische Kernfrage, wie deren Erfolge oder Tatsachen zugerechnet werden. Dafür kann die Zurechnungslehre herangezogen werden. ${ }^{632}$ Hierbei spielt nicht nur die Frage des Umgangs dieser Attribute bei einer Künstlichen Intelligenz eine Rolle - die ohnehin nur über zwei der drei Attribute verfügen kann - sondern auch der

629 S. o., Vom Sein und Sollen (S. 64).

630 S. o., Explainable AI Ansätze (S. 65).

631 S. o., Wissen und dessen Repräsentation (S. 66).

632 S. o., Grundlagen der Zurechnungslehre (S. 72). 
Umgang der Attribute bei den beteiligten Personen, insbesondere im Vergleich zu bisherigen (herkömmlichen) technischen Phänomen. Im allgemeinen Zivilrecht sind dies die Auswirkungen auf das Vertrags- und Deliktsrecht bzw. auf die Rechtsgeschäftslehre und das Haftungsrecht, in welchen insbesondere die Folgen des Verhaltens einer Künstlichen Intelligenz von Relevanz sind. Der Zurechnung des Wissens kommt dabei nur eine untergeordnete Rolle zu, da sich die Frage nach dem Wissen einer Künstlichen Intelligenz nicht von der Frage des Wissens anderer Computersoftware und damit nicht von bisherigen technischen Phänomenen unterscheidet. ${ }^{633}$

10. Die Frage, ob Künstlicher Intelligenz eine (Teil-)Rechtsfähigkeit zukommen soll, muss frei von philosophischen Implikationen - im Sinne einer reinen Rechtslehre - beantwortet werden. Grundsätzlich spricht die Dogmatik der Rechtsfähigkeit nicht dagegen, entsprechenden Entitäten eine solche Rechtsfähigkeit zukommen zu lassen. Allerdings muss sich die (Teil-)Rechtsfähigkeit daran messen lassen, ${ }^{634} \mathrm{ob}$ sie einen Mehrwert bietet oder ob eine solche Lösung an der Problemlage vorbei geht und damit die Herausforderungen - insbesondere in der Rechtsgeschäftslehre und im Haftungsrecht - an anderer Stelle liegen. ${ }^{635}$

11. In der Rechtsgeschäftslehre ist zwischen zwei Fällen der Funktion einer Künstlichen Intelligenz zu unterscheiden: Im ersten Fall ist sie nur Hilfsmittel des Erklärenden und daher nicht mehr als ein besseres Eingabegerät, wie beispielsweise spracherkennende Assistenten. Diese Fälle lassen sich ohne große Schwierigkeiten mit den bestehenden Ansätzen der Rechtsgeschäftslehre lösen, wenn entsprechende subjektive Merkmale beim Benutzer vorliegen. Bei einer entsprechenden Handlung, die als Erklärung aufgefasst werden kann, liegt entweder ein Wille des Erklärenden vor oder der Erklärende hat einen Rechtsschein geschaffen, der eine entsprechende Rechtsscheinhaftung begründet. Liegt eine (menschliche) Handlung vor, die zwar eine Erklärung des Systems zur Folge hat, selbst aber an den Voraussetzungen einer Willenserklärung scheitert, liegt schon keine Willenserklärung mehr vor. Das ist der Fall, wenn die Handlung des Menschen nicht als rechtserhebliche Erklärung aufgefasst werden kann, weil kein Erklärungsbewusstsein und auch keine Erklärungsfahrlässigkeit

633 S. o., Wissenszurechnung (S. 133).

634 S. o., Beteiligte Rechtssubjekte (S. 75).

635 S. o., Rechtssubjektivierung von Künstlicher Intelligenz oder von auf sie bezogenen Entitäten (S. 77). 
vorliegt. ${ }^{636}$ Bereits hier zeigt sich das Problem, dass Erklärungen der Künstlichen Intelligenz nicht immer auf einen Willen des Benutzers zurückgeführt werden können. Der zweite Fall geht über die reine Stellung als Hilfsmittel hinaus. ${ }^{637}$ Dann entscheiden Künstliche Intelligenzen autonom, wann eine Erklärung erzeugt und abgegeben wird. Dies sind die sog. »autonomen Systeme«, die allerdings nur einen Spezialfall einer Künstlichen Intelligenz darstellen. Hier besteht das Problem, dass keine Handlung des Benutzers mehr vorliegt, die schon aus Sicht eines objektiven Dritten eine Willenserklärung wäre, selbst dann, wenn die Erklärung einem späteren Willen des Benutzers entspricht. Damit liegen die Herausforderungen bei autonomen Systemen schon auf der Ebene der objektiven Voraussetzung an eine Willenserklärung und nicht erst bei den subjektiven Voraussetzungen. $\mathrm{Da}$ eine analoge Anwendung der Regelungen der Blanketterklärung mangels gleichartiger Lage ausfällt, ${ }^{638}$ bleibt nur noch die Inbetriebnahme des Systems. Diese kann in der Regel aber nicht als Willenserklärung aufgefasst werden. Nur in wenigen und sehr speziellen Ausnahmefällen kann ein anderes Ergebnis vorliegen. Zu denken ist hier an Systeme, die von einem Angehörigen eines Verkehrskreises mit erhöhter Fachkenntnis mit einem auf nur solche autonome Erklärungserzeugung gerichteten Zweck betrieben werden, ${ }^{639}$ beispielsweise bei Systemen, die eine autonome Annahme erklären. ${ }^{640}$ Bei Verbrauchern, die beispielsweise einen autonomen Kühlschrank in Betrieb nehmen, fehlt es an diesen Voraussetzungen allerdings vollständig. ${ }^{641}$

12. Da sich die Willenserklärung schon begrifflich in »Wille« und »Erklärung« aufteilt und das Attribut des Willens bei Künstlicher Intelligenz nicht vorliegt, ist deren Verhältnis essenziell für die Frage, ob überhaupt eine Willenserklärung einer Künstlichen Intelligenz vorliegen kann. Zwar ist nach wie vor die dogmatische Frage der Willenserklärung nicht abschließend geklärt, ${ }^{642}$ allerdings wird in der Regel an einem Willenselement - zumindest am Handlungswillen - festgehalten. Daraus folgt, dass eine Erklärung einer Künstlichen Intelligenz nicht als eigene Willenserklärung der Künstlichen Intelligenz aufgefasst werden kann und somit eine (Teil-) Rechtsfähigkeit von Künstlichen Intelligenzen in der Rechtsgeschäftslehre

636 S. o., Künstliche Intelligenz als Hilfsmittel (S. 100).

637 S. o., Künstliche Intelligenz als autonomer »Erklärender« (S. 104).

638 S. o., Vorliegen einer Blanketterklärung (S. 111).

639 S. o., Zwischenergebnis(S. 111).

640 S. o., Annahmen durch autonome Systeme (S. 108).

641 S. o., Angebote durch autonome Systeme (S. 105).

642 S. o., Dichotomie von Wille und Erklärung (S. 83). 
die Probleme nicht lösen kann. Selbst mit einer (Teil-)Rechtsfähigkeit wären Erklärungen der Künstlichen Intelligenz somit keine Willenserklärung. Fraglich ist vielmehr, welche Elemente eine Willenserklärung umfassen soll, genauer, wie subjektive und objektive Merkmale zu bestimmen sind. ${ }^{643}$

13. Eine verschuldensabhängige Haftung der Künstlichen Intelligenz für eigenes Verhalten kommt nicht in Frage. Entgegen einer natürlichen Person kann eine Künstliche Intelligenz - mangels Willens - a priori kein Verschulden treffen. Vielmehr folgt aus der deterministischen Eigenschaft, dass das gleiche Verhalten in der gleichen Situation immer wieder gezeigt werden würde. Ein vergleichbares »Lernen« aus der Situation - wie es beim menschlichen Verhalten der Fall ist - kann nicht entstehen, da eine Künstliche Intelligenz keinen entsprechenden (moralischen) Vergleichsmaßstab hat. Über Kategorien wie »moralisch richtig« oder »moralisch falsch« verfügt eine Künstliche Intelligenz schlichtweg nicht. ${ }^{644}$

14. Bei den auf Künstliche Intelligenz bezogenen Akteuren (Benutzer, Betreiber und Hersteller) kommt eine Haftung für eigenes Verhalten nur in zwei Fällen in Betracht: Entweder wenn eine verschuldensunabhängige Haftung besteht oder wenn ein schuldhaftes Verhalten der Akteure stattfindet. ${ }^{645}$ Besondere, auf Künstliche Intelligenz bezogene, verschuldensunabhängige Haftungsansätze bestehen nicht, sodass eine solche Haftung nur dann in Frage kommt, wenn eine andere spezialgesetzliche verschuldensunabhängige Haftung greift. Für den Hersteller ist dies insbesondere die Produkthaftung des ProdHaftG, deren Reichweite und Exkulpationsmöglichkeiten allerdings der deliktischen Produkthaftung nahe kommen und die daher kaum einen Mehrwert bietet. ${ }^{646}$ Bei der verschuldensabhängigen Haftung bestehen die Herausforderungen vor allem darin, zumindest eine Fahrlässigkeit der einzelnen Akteure zu begründen. Sie kommt allerdings nur für Fehler und nicht für unvorhersehbares Verhalten in Frage, da letzteres - mangels Vorhersehbarkeit - keine Fahrlässigkeit begründen kann. Wie der jeweilige Sorgfaltsmaßstab bei den Akteuren zu bestimmen ist und damit welchem Verkehrskreis die Akteure zuzuordnen sind, orientiert sich am Einzelfall, je nachdem, welchen Einfluss, welches Fachwissen und welchen Einblick der jeweilige Akteur auf und in die Künstliche Intelligenz hat. Bei einem Verbraucher ist der Sorgfalts-

643 S. o., Folgen (S. 112).

644 S. o., Haftung der Künstlichen Intelligenz für eigenes Verhalten (S. 117).

645 S. o., Haftung für eigenes Verhalten der Beteiligten (S. 119).

646 S. o., Verschuldensunabhängige Haftung (S. 125). 
maßstab in der Regel niedriger anzusetzen als bei einem gewerblichen Anwender. Auf Seiten des Herstellers sind die Besonderheiten des deliktischen Produkthaftungsrechts zu beachten, sodass bei zu erkennenden Fehlern auf den hohen Stand der Wissenschaft und Technik abzustellen ist und im Rahmen des Zumutbaren entsprechende Fehler der Künstlichen Intelligenz zu suchen sind. Insbesondere ist es aufgrund des geringen Implementierungsaufwands einem Hersteller zumutbar, Methoden der Explainable AI (xAI) anzuwenden, woraus folgt, dass die Nichtanwendung entsprechender Methoden bei einem damit auffindbaren Fehler als fahrlässig zu bewerten ist und damit eine Haftung begründen kann. ${ }^{647}$

15. Das Verhalten der Künstlichen Intelligenz als fremdes Verhalten zu verstehen, für welches eingestanden werden muss, ist de lege lata nicht möglich; eine Anwendung des $§ 278$ BGB scheitert nach wie vor am Verschulden. ${ }^{648}$ Zwar ist eine analoge Anwendung des $\S 831$ BGB diskutabel, ${ }^{649}$ sodass ein Verschulden der Künstlichen Intelligenz nicht mehr von Interesse ist, allerdings entsteht wieder ein Verschuldensproblem auf Seiten der Akteure. Zudem zeigt sich - wie schon beim Verschulden der beteiligten Akteure - dass bei entsprechenden Ansätzen nach wie vor Lücken bei der Begründung der Kausalität bestehen. Bei Anwendung der (verhaltensbezogenen) Adäquanztheorie kann keine Haftung (kausal) begründet werden, wenn unvorhersehbares Verhalten vorliegt. ${ }^{650}$

16. Im Gesamten zeigt sich, dass eine Rechtsfähigkeit einer Künstlichen Intelligenz - unabhängig davon, wie weit diese geht - keinen Mehrwert bietet. Willenserklärungen und Vertragsschluss scheitern derzeit am fehlenden Willen einer Künstlichen Intelligenz. Auch mit der Zuweisung einer (Teil-)Rechtfähigkeit wird ein Wille nicht entstehen, da die rechtstatsächliche Wesenseigenschaft der Künstlichen Intelligenz die gleiche bleibt. ${ }^{651}$ Gleiches gilt im Haftungsrecht: Auch hier würde eine (Teil-) Rechtsfähigkeit ein mögliches Verschulden von Künstlicher Intelligenz nicht begründen können, da auch hier der (eigene) Wille fehlt. Zielführender sind verschuldensunabhängige Ansätze, die - beispielsweise als Gefährdungshaftung - auch die Probleme in der Kausalität bei unvorhersehbarem Verhalten überwinden. Damit verkommt die Frage der (Teil-)

647 S. o., Fahrlässigkeit (S. 120).

648 S. o., Künstliche Intelligenz als Erfüllungsgehilfe (S. 126).

649 S. o., Künstliche Intelligenz als Verrichtungsgehilfe (S. 127).

650 S. o., Adäquanztheorie (S. 130).

651 S. o., Folgen (S. 112). 
Rechtsfähigkeit zur Frage, wessen Vermögen in derartigen Fällen heranzuziehen ist. ${ }^{652}$

652 S. o., Folgen (S. 132). 


\section{Literaturverzeichnis}

Agarwal, Manish, Fuzzy Logic Control of Washing Machines, Paper 2011

Aho, Alfred V./Lam, Monica S./Sethi, Ravi/Ullman, Jeffry D., Compiler, 2. Aufl. München 2008

Alcorn, Michael A./Li, Qi/Gong, Zhitao/Wang, Chengfei/Mai, Long/Ku, Wei-Shinn/Nguyen, Anh, Strike (with) a Pose: Neural Networks Are Easily Fooled by Strange Poses of Familiar Objects, Paper 2019 [arXiv: 1811.11553] (zitiert: Alcorn/Li/Gong et al.)

Alkhateeb, Faisal/Al Maghayreh, Eslam/Aljawarneh, Shadi, A Multi Agent-Based System for Securing University Campus: Design and Architecture, in: 2010 International Conference in Intelligent Systems, Modelling and Simulation, Washington DC 2010, S. 75-79 (zitiert: Alkhateeb/Al Maghayreh/Aljawarneh, in: ICISMS)

Ansari, Abdul Quaiyum, The Basics of Fuzzy Logic, Computer Education 88 (1998), 5-9.

Arrieta, Alejandro Barredo/Días-Rodríguez, Natalia/Del Ser, Javier/Bennetot, Siham Tabik/Barbado, Alberto/Garcia, Salvador/Gil-Lopez, Sergio/Molina, Daniel/Benjamins, Richard/Chatila, Raja/Herrera, Francisco, Explainable Artificial Intelligence (XAI): Concepts, Taxonomies, Opportunities and Challenges toward Responsible AI, Paper 2019 [arXiv:1910.10045] (zitiert: Arrieta/ Días-Rodríguez/Del Ser et al.)

Ashby, Ross, Requisite variety and its implications for the control of complex systems, in: Cybernetica 1:2 (1958), 83-99.

Aspray, William, Computing Before Computers, Ames (IA) 1990 (zitiert: Aspray/Bearbeiter)

Bach, Sebastian/Binder, Alexander/Montavon, Grégoire/Klauschen, Frederick/Müller, Klaus-Robert/Samek, Wojciech, On Pixel-Wise Explanations for Non-Linear Classifier Decisions by Layer-Wise Relevance Propagation, PLoS ONE 10(7): e0130140 (2015), 1-46 (zitiert: Bach/Binder/Montavon et al.)

Baehrens, David/Schroeter, Timon/Harmeling, Stefan/Kawanabe, Motoaki/Hansen, Katja/Müller, Klaus-Robert, How to Explain Individual Classification Decisions, Journal of Machine Learning Research 11 (2010), 1803-1831 (zitiert: Baehrens/Schroeter/Harmeling et al.)

Bauernhansl, Thomas, Die Vierte Industrielle Revolution - Der Weg in ein wertschaffendes Produktionsparadigma, in: Vogel-Heuser, Birgit/Bauernhansl, Thomas/ten Hompel, Michael, Handbuch Industrie 4.0, Bd. 4, 2. Aufl. Berlin 2017, S. 1-33 (zitiert: Bauernhansl, in: Vogel-Heuser/Bauernhansl/ten Hompel)

beck-online Großkommentar zum Zivilrecht, Stand: 01.04.2020, München 2020 (zitiert: BeckOGK/Bearbeiter)

Beck'scher Online Kommentar zum Bürgerlichen Gesetzbuch, 54. Edition, Stand: 01.05.2020, München 2020 (zitiert: BeckOK/Bearbeiter) 
Bellamy, Rachel K. E./Dey, Kuntal/Hind, Michael/Hoffmann, Samuel C./Houde, Stephanie/Kannan, Kalapriya/Lohia, Pranay/Martino, Jacquelyn/Metha, Sameep/Mojsilovic, Aleksandra/Naga, Seema/Ramamurthy, Karthikeyan Natesan/Richards, John/ Saha, Diptikalyan/Sattigeri, Prasanna/Singh, Moninder/Varshney, Kush R./Zhang, Yunfeng, AI Fairness 360: An Extensible Toolkit for Detecting, Understanding, and Mitigating unwanted Algorithmic Bias, Paper 2018 [arXiv:1810.01943] (zitiert: Bellamy/Dey/Hind et al.)

Ben-Nun, Tal/Hoefler, Torsten, Demystifying Parallel and Distributed Deep Learning: An In-Depth Concurrency Analysis, Paper 2018 [arXiv:1802.09941]

Beuthien, Volker, Zur Begriffsverwirrung im deutschen Gesellschaftsrecht, JZ 2003, 715-722

Beuthien, Volker, Zur Grundlagenungewissheit des deutschen Gesellschaftsrechts, NJW 2005, 855-860

Binder, Alexander/Bach, Sebastian/Montavon, Grégoire/Müller, Klaus-Robert/Samek, Wojciech, Layer-Wise Relevance Propagation for Deep Neural Network Architectures, Information Science and Applications 2016, 913-922 (zitiert: Binder/Bach/ Montavon et al.)

Bodenstedt, Kai, »Alles für einen Euro«? - Abgrenzung von Zugangsbestätigung und Annahmeerklärungen im Internet, MMR 2004, 719-723

Boemke, Burkhard/Ulrici, Bernhard, BGB Allgemeiner Teil, 2. Aufl. Berlin 2014

Borges, Georg, Haftung für Identitätsmissbrauch im Online-Banking, NJW 2012, 2385-2389

Borges, Georg, Haftung für selbstfahrende Autos, CR 2016, 272-280

Borges, Georg, Rechtsscheinhaftung im Internet, NJW 2011, 2400-2403

Bork, Reinhard, Allgemeiner Teil des Bürgerlichen Gesetzbuchs, 4. Aufl. Tübingen 2016

Brehmer, Nikolaus, Willenserklärung und Erklärungsbewusstsein - BGHZ 91, 324, JuS 1986, 440-445

Brox, Hans/Walker, Wolf-Dietrich, Allgemeines Schuldrecht, 44. Aufl. München 2020 (zitiert: Brox/Walker, Allg. SchuldR.)

Brox, Hans/Walker, Wolf-Dietrich, Besonderes Schuldrecht, 44. Aufl. München 2020 (zitiert: Brox/Walker, Bes. SchuldR.)

Broy, Manfred, Informatik - Eine grundlegende Einführung (Bd. 1: Programmierung und Rechnerstrukturen), 2. Aufl. Berlin 1998

Brüggemeier, Gert, Grundstrukturen des zivilrechtlichen Delikts, AcP 219 (2019), 771-817

Brüggemeier, Gert, Produkthaftung und Produktsicherheit, ZHR 152 (1988), 511-536

Brüggemeier, Gert, Produzentenhaftung nach $\S 823$ Abs. 1 BGB - Bestandsaufnahme und Perspektiven weiterer judizieller Rechtsentwicklung, WM 1982, 1294-1309

Buck, Petra, Wissen und juristische Person, Tübingen 2001

Bünau, Paul von, Künstliche Intelligenz im Recht - Möglichkeiten und Mythos, in: Breidenbach, Stefan/Glatz, Florian, Rechtshandbuch Legal Tech, München 2018, S. 47-90 (zitiert: v. Bünau, in: Breidenbach/Glatz) 
Buxmann, Peter/Schmidt, Holger, Künstliche Intelligenz: Mit Algorithmen zum wirtschaftlichen Erfolg, Berlin 2019

Canaris, Claus-Wilhelm, Grundstrukturen des deutschen Deliktsrechts, VersR 2005, 577-584

Clemens, Rudolf, Die elektronische Willenserklärung - Chancen und Gefahren, NJW 1985, 1998-2005

Cormen, Thomas H./Leiserson, Charles E./Rivest, Ronald/Stein, Clifford, Algorithmen - Eine Einführung, 3. Aufl. München 2010

Cornelius, Kai, Vertragsschluss durch autonome elektronische Agenten, MMR 2002, 353-358

Czarnul, Pawel, Benchmarking Parallel Chess Search in Stockfish on Intel Xeon and Intel Xeon Phi Processors, in: Shi, Yong/Fu, Haohuan/Tian, Yingjie/Krzhizhanovskaya, Valeria V./Lees, Michael H./Dongarra, Jack/Sloot, Peter M. A., Computational Science - ICCS 2018, Wuxi 2018, S. 457-464 (zitiert: Czarnul, in: Shi et al.)

Czerner, Frank, Der strafrechtlich-normative Schuldbegriff zwischen Willensfreiheit und neurobiologischem Determinismus (I), ArchKimm 218 (2006), 65-88

Dafflon, Baudouin/Vilca, Jose/Gechter, Franck/Adouane, Lounis, Adaptive Autonomous Navigation using Reactive Multi-agent System for Control Law Merging, Procedia Computer Science 51 (2015), 423-432

Dassow, Jürgen, Logik für Informatiker, Stuttgart 2005

Davies, John/Warren, Paul, Knowledge Management in Large Organisations, in: Domingue, John/Fensel, Dieter/Hendler, James A., Handbook of Semantic Web Technologies, Berlin 2011, S. 737-786 (zitiert: Davies/Warren, in: Domingue/Fensel/ Hendler)

Denga, Michael, Deliktische Haftung für künstliche Intelligenz - Warum die Verschuldenshaftung des BGB auch künftig die bessere Schadensausgleichsordnung bedeutet, CR 2018, 69-78

Der neue Georges, Ausführliches Handwörterbuch Lateinisch - Deutsch, Bd. 1 (A-H) Darmstadt 2013; Bd. 2 (I-Z) Darmstadt 2013

Dettling, Heinz-Uwe/Krüger, Stefan, Digitalisierung, Algorithmisierung und Künstliche Intelligenz im Pharmarecht, PharmR 2018, 513-522

Deutsch, Erwin, Der Zurechnungsgrund der Produzentenhaftung, VersR 1988, 1197-1201

Deutsch, Erwin, Die Fahrlässigkeit im neuen Schuldrecht, AcP 202 (2002), 889-911

DIN e.V., Künstliche Intelligenz - Mit Normung und Standardisierung innovationsfreundliche Rahmenbedingungen schaffen, Positionspapier, Berlin 2019 (zitiert: DIN, Künstliche Intelligenz)

Dreier, Thomas/Schulze, Gernot, Urheberrechtsgesetz, 6. Aufl. München 2018 (zitiert: Dreier/Schulze/Bearbeiter)

Ehinger, Patrick/Stiemerling, Oliver, Die urheberrechtliche Schutzfähigkeit von Künstlicher Intelligenz am Beispiel von Neuronalen Netzen, CR 2018, 761-770

Ehmann, Horst, Informationsschutz und Informationsverkehr im Zivilrecht, AcP 188 (1988), 230-380 
Engels, Barbara/Goecke, Henry, IW-Analysen 130 - Big Data in Wirtschaft und Wissenschaft, Köln 2019

Enneccerus, Ludwig/Nipperdey Hans Carl, Allgemeiner Teil des Bürgerlichen Rechts, Erster Halbband (Allgemeine Lehren, Personen, Rechtsobjekte), 15. Aufl. Tübingen 1959

Ertel, Wolfgang, Grundkurs Künstliche Intelligenz, 4. Aufl. Wiesbaden 2016

Esser, Robert/Reißmann, Ludwig, Einsatz künstlicher Intelligenz zur Suizidprävention im Justizvollzug, JZ 2019, 975-982

Etzkorn, Philipp, Bedeutung der »Entwicklungslücke« bei selbstlernenden Systemen Rechtliche Fragen zur fortdauernden Softwareentwicklung durch maschinelles Lernen im Praxiseinsatz, MMR 2020, 360-365

Faust, Florian, BGB AT: Nutzung eines fremden eBay-Mitgliedskontos (Anmerkung zu BGH, U. v. 11.05.2011 - VIII ZR 289/09), JuS 2011, 1027-1030

Federrath, Hannes, Herausforderungen des technologischen Wandels an das Recht aus Sicht der Technik, ZGE/IPJ 6 (2014), 271-278

Fischer, Peter/Hofer, Peter, Lexikon der Informatik, 14. Aufl. Berlin 2008

Fischerauer, Gerhard, Mechatronik, in: Rieg, Frank/Steinhilper, Rolf, Handbuch Konstruktion, München 2012, S. 291-312 (zitiert: Fischerauer, in: Rieg/Steinhilper)

Flume, Werner, Allgemeiner Teil des Bürgerlichen Rechts, Bd. 2 (Das Rechtsgeschäft), 4. Aufl. Berlin 1992

Foerster, Max, Automatisierung und Verantwortung im Zivilrecht, ZfPW 2019, 418-435

Frempong, Nana K./Nicholas, Nimo/Boateng, Maxwell, Decision Tree as a Predictive Modeling Tool for Auto Insurance Claims, International Journal of Statistics and Applications 2017, 117-120

Fritzsche, Jörg, Der Abschluss von Verträgen, §§ 145 ff. BGB, JA 2006, 674-681

Fritzsche, Jörg/Malzer, Hans M., Ausgewählte zivilrechtliche Probleme elektronisch signierter Willenserklärungen, DNotZ 1995, 3-26

Fuchs, Maximilian/Baumgärtner, Alex, Ansprüche aus Produzentenhaftung und Produkthaftung, JuS 2011, 1057-1063

Geis, Ivo, Die digitale Signatur, NJW 1997, 3000-3004

Gitter, Rotraud/Roßnagel, Alexander, Rechtsfragen mobiler Agentensysteme im ECommerce, K\&R 2003, 64-72

Gliozzo, Alfio/Ackerson, Chris/Bhattacharya, Rajib/Goering, Addison/Jumba, Albert/ Kim, Seung Yeon/Krishnamurty, Laksh/Lam, Tanh/Littera, Angelo/McIntosh, Iain/ Murthy, Srini/Ribas, Marcel, Building Cognitive Applications with IBM Watson Services (Volume 1, Getting Started), Poughkeepsie 2017 (zitiert: Gliozzo/Ackerson/ Bhattacharya et al.)

Gomille, Christian, Kreative künstliche Intelligenz und das Urheberrecht, JZ 2019, 969-975

Goos, Gerhard, Vorlesungen über Informatik (Bd. 1: Grundlagen und funktionales Programmieren), 3. Aufl. Berlin 2000

Graevenitz, Albrecht von, »Zwei mal Zwei ist Grün« - Mensch und KI im Vergleich, ZRP 2018, 238-241 
Grapentin, Justin, Die Erosion der Vertragsgestaltungsmacht durch das Internet und den Einsatz Künstlicher Intelligenz, NJW 2019, 181-185

Grapentin, Justin, Vertragsschluss und vertragliches Verschulden beim Einsatz von Künstlicher Intelligenz und Softwareagenten, Baden-Baden 2018

Greiner, Stefan/Kalle, Ansgar, Ungeklärte Fragen des Wirksamwerdens empfangsbedürftiger Willenserklärungen - im Grundsatz und bei der Verwendung digitaler Kommunikationswege, JZ 2018, 535-541

Grigoleit, Hans-Christoph, Besondere Vertriebsformen im BGB, NJW 2002, 11511158

Grigoleit, Hans-Christoph, Zivilrechtliche Grundlagen der Wissenszurechnung, ZHR 181 (2017), 160-202

Grigoleit, Hans-Christoph/Herresthal, Carsten, BGB Allgemeiner Teil, 3. Aufl. München 2015

Gronau. Norbert/Bahrs, Julian/Vladova, Gergana/Baumgrass, Anne/Meuthrath, Benedikt/Peters, Kirstin, Anwendungen und Systeme für das Wissensmanagement - Ein aktueller Überblick, 3. Aufl. Berlin 2009 (zitiert: Gronau/Bahrs/Vladova et al.)

Grote, Karl-Heinrich/Feldhusen, Jörg, Dubbel - Taschenbuch für den Maschinenbau, 24. Aufl. Berlin 2014 (zitiert: Grote/Feldhusen/Bearbeiter, Dubbel)

Güting, Ralf Hartmut, Datenstrukturen und Algorithmen, Stuttgart 1992

Haberstumpf, Helmut, Der urheberrechtliche Schutz von Computerprogrammen, in: Lehmann, Michael, Rechtsschutz und Verwertung von Computerprogrammen, 2. Aufl. Köln 1993, S. 69-167 (zitiert: Haberstumpf, in: Lehmann)

Hacker, Philipp, Immaterialgüterrechtlicher Schutz von KI-Trainingsdaten, GRUR 2020, 1025-1033

Hacker, Philipp, Verhaltens- und Wissenszurechnung beim Einsatz von Künstlicher Intelligenz, RW 2018, 243-288

Hagan, Martin T./Demuth, Howard B./Beale Mark H./De Jesús, Orlando, Neural Network Design, 2nd Edition Stillwater 2014

Hanslmeier, Arnold, Einführung in die Astronomie und Astrophysik, 3. Aufl. Berlin 2013

Harke, Jan Dirk, Sklavenhaltung in Rom, in: Gless, Sabine/Seelmann, Kurt, Intelligente Agenten und das Recht, Baden-Baden 2016, S. 97-117 (zitiert: Harke, in: Gless/Seelmann)

Hartmann, Frank/Prinz, Matthias, Immaterialgüterrechtlicher Schutz von Systemen Künstlicher Intelligenz, WRP 2018, 1431-1438

Hattenhauer, Hans, »Person« - Zur Geschichte eines Begriffs, JuS 1982, 405-411

Hauck, Ronny/Cevc, Baltasar, Patentschutz für Systeme Künstlicher Intelligenz, ZGE/IPJ 11 (2019), 135-169

Heidel, Thomas/Hüßtege, Rainer/Mansel, Heinz-Peter/Noack, Ulrich, NomosKommentar BGB Bd. 1 (Allgemeiner Teil, EGBGB), 3. Aufl. Baden-Baden 2016 (zitiert: Heidel/Hüßtege/Mansel/Noack/Bearbeiter)

Hellfeld, Axel von, Sind Algorithmen schutzfähig?, GRUR 1989, 471-485 
Henderson, Harry, Encyclopedia of Computer Science and Technology, Revised Edition New York 2009

Herberger, Maximilian, »Künstliche Intelligenz« und Recht, NJW 2018, 2825-2829

Herresthal, Carsten, Anmerkung zu BGH, U. v. 11.05.2011 - VIII ZR 289/09, JZ 2011, 1171-1174

Herresthal, Carsten, Anscheinsbeweis und Rechtsscheinhaftung beim Online-Banking - Zugleich Besprechung von BGH, Urteil v. 26.1.2016 - XI ZR 91/14, JZ 2017, 2835

Herresthal, Carsten, Die Haftung bei Accountüberlassung und Accountmissbrauch im Bürgerlichen Recht, in: Taeger, Jürgen/Wiebe, Andreas, Tagungsband Herbstakademie 2008 - Von AdWords bis Social Networks - Neue Entwicklungen im Informationsrecht, Edewecht 2008, S. 21-46 (zitiert: Herresthal, in: Taeger/Wiebe)

Herwig, Volker, Zugang und Zustellung in elektronischen Medien, MMR 2001, 145-149

Hetmank, Sven/Lauber-Rönsberg, Anne, Künstliche Intelligenz - Herausforderungen für das Immaterialgüterrecht, GRUR 2018, 574-582

Heun, Sven-Erik, Die elektronische Willenserklärung, CR 1994, 595-600

Heussen, Benno, Unvermeidbare Softwarefehler - Neue Entlastungsmöglichkeiten für den Hersteller, CR 2004, 1-10

Historisch-kritischer Kommentar zum BGB, Bd. 1 (Allgemeiner Teil, §§ 1-240), Tübingen 2003 (zitiert: HKK-BGB/Bearbeiter)

Hobbes, Thomas, Leviathan. Erster und zweiter Teil, Stuttgart 1996 (Nachdruck)

Hoch, Veronica R. S., Anwendung Künstlicher Intelligenz zur Beurteilung von Rechtsfragen im unternehmerischen Bereich - Zulässigkeit, Grenzen und Haftungsfragen beim Einsatz von Legal Robots, AcP 219 (2019), 646-702

Hoeren, Thomas/Niehoff, Maurice, KI und Datenschutz - Begründungserfordernisse automatisierter Entscheidungen, RW 2018, 47-66

Hoeren, Thomas/Sieber, Ulrich/Holznagel, Bernd, Handbuch Multimedia-Recht, 51. Ergänzungslieferung (Februar 2020), München 2020 (zitiert: Hoeren/Sieber/Holznagel/Bearbeiter)

Hoffmann, Dirk W., Grenzen der Mathematik: Eine Reise durch die Kerngebiete der Logik, 3. Aufl. Berlin 2018 (zitiert: Hoffmann, Grenzen der Mathematik)

Hoffmann, Dirk W., Grundlagen der Technischen Informatik, München 2007 (zitiert: Hoffmann, Technische Informatik)

Hoffmann, Dirk W., Theoretische Informatik, 2. Aufl. München 2011 (zitiert: Hoffmann, Theoretische Informatik)

Hofmann, Franz, Grundsatz der Technikneutralität im Urheberrecht? - Zugleich Gedanken zu einem more technological approach, ZGE/IPJ 8 (2016), 482-512

Hornby, Gregory S./Lohn, Jason D./Linden, Derek S., Computer-Automated Evolution of an X-Band Antenna for NASA's Space Technology 5 Mission, Evolutionary Computation 19 (2011), 1-23

Hromkovič, Juri, Theoretische Informatik, 4. Aufl. Wiesbaden 2011

Im, Hwan-Jae, Die Entwicklung eines europäischen Schlüsselwortes: Intelligenz und seine Bedeutung in der Wissenschaftssprache, Dissertation Bonn 1975 
Isern, David/Moreno, Antonio, A Systematic Literature Review of Agents Applied in Healthcare, J Med Syst (2016) 40:43, 1-14

Jakl, Bernhard, Das Recht der Künstlichen Intelligenz - Möglichkeiten und Grenzen zivilrechtlicher Regulierung, MMR 2019, 711-715

Janal, Ruth, Die Flugbuchung für »Mr. noch unbekannt« und andere widersprüchliche Erklärungen im elektronischen Geschäftsverkehr, AcP 215 (2015), 830-854

Jarosch, Helmut, Information Retrieval und Künstliche Intelligenz, Wiesbaden 2007

Jauernig, Othmar, Bürgerliches Gesetzbuch, 17. Aufl. 2018 (zitiert: Jauernig/ Bearbeiter)

Jiang, Yi, Credit Scoring Model Based on the Decision Tree and the Simulated Annealing Algorithm, in: 2009 WRI World Congress on Computer Science and Information Engineering (Proceedings), S. 18-22, Los Angeles 2009 (zitiert: Jiang, in: WRI)

John, Uwe, Grundsätzliches zum Wirksamwerden empfangsbedürftiger Willenserklärungen, AcP 184 (1984), 385-412

Käde, Lisa/Maltzan, Stephanie von, Die Erklärbarkeit von Künstlichen Intelligenz (KI) - Entmystifizierung der Black Box und Chancen für das Recht, CR 2020, 66-72

Karloff, Howard J., Randomized Algorithms and Pseudorandom Numbers, Journal of the Association for Computing Machinery 40 (1993), 454-476

Karmon, Danny/Zoran, Daniel/Goldberg, Yoav, LaVAN: Localized and Visible Adversarial Noise, Paper 2018 [arXiv: 1801.02608]

Karp, Richard M., An introduction to randomized algorithms, Discrete Applied Mathematics 34 (1991), 165-200

Karpfinger, Christian/Kiechle, Hubert, Kryptologie: Algebraische Methoden und Algorithmen, Wiesbaden 2010

Kaufmann, Arthur/Hassemer, Winfried/Neumann, Ulfrid, Einführung in die Rechtsphilosophie und Rechtstheorie der Gegenwart, 8. Aufl. Heidelberg 2011 (zitiert: Kaufmann/Hassemer/Neumann/Bearbeiter)

Kellmann, Christof, Grundprobleme der Willenserklärung, JuS 1971, 609-617

Kersten, Jens, Menschen und Maschinen - Rechtliche Konturen instrumentaler, symbiotischer und autonomer Konstellationen, JZ 2015, 1-8

Keßler, Oliver, Intelligente Roboter - neuen Technologien im Einsatz - Voraussetzungen und Rechtsfolgen des Handelns informationstechnischer Systeme, MMR 2017, 589-594

Kindermann. Manfred, Was ist Computer-Software?, ZUM 1985, 2-10

Kirn, Stefan/Müller-Hengstenberg, Claus D., Intelligente (Software-)Agenten: Von der Automatisierung zur Autonomie? - Verselbstständigung technischer Systeme, MMR 2014, 225-232

Klaeren, Herbert/Sperber, Michael, Vom Problem zum Programm - Architektur und Bedeutung von Computerprogrammen, 3. Aufl. Stuttgart 2001

Kleene, Stephen Cole, Introduction to Metamathematics, Amsterdam 1952/1976

Klingbeil, Stefan, Der Begriff der Rechtsperson, AcP 217 (2017), 848-885 
Klingbeil, Stefan, Schuldnerhaftung für Roboterversagen - Zum Problem der Substitution von Erfüllungsgehilfen durch Maschinen, JZ 2019, 718-725

Klingmüller, Ernst, Einführung, in: Möglichkeiten der Wissenszurechnung - Karlsruher Forum 1994 (VersR Sonderheft), S. 1-3 (zitiert: Klingmüller, in: Möglichkeiten der Wissenszurechnung)

Kluge, Etymologisches Wörterbuch der deutschen Sprache, 24. Aufl. Berlin 2002

Kocarev, Ljupco/Jakimoski, Goce/Tasev, Zarko, Chaos and Pseudo-Randomness, LNCIS 292 (2003) 247-263

Köhler, Helmut, BGB - Allgemeiner Teil, 43. Aufl. München 2019

Köhler, Helmut, Die Problematik automatisierter Rechtsvorgänge, insbesondere von Willenserklärungen, AcP 182 (1982), 126-171

Köhler, Helmut/Bornkamm, Joachim/Feddersen, Jörn, Gesetz gegen den unlauteren Wettbewerb, 38. Aufl. München 2020 (zitiert: Köhler/Bornkamm/Feddersen/Bearbeiter)

Köhler, Helmut/Fetzer, Thomas, Recht des Internet, 8. Aufl. Heidelberg 2016

Konertz, Roman/Schönhof, Raoul, Erfindungen durch Computer und künstliche Intelligenz - eine aktuelle Herausforderung für das Patentrecht?, ZGE/IPJ 10 (2018), 379412

Kötz, Hein/Wagner, Gerhard, Deliktsrecht, 13. Aufl. München 2016

Krempel, Rasmus, Netze, Karten, Irrgärten: Graphenbasierte explorative Ansätze zur Datenanalyse und Anwendungsentwicklung in den Geisteswissenschaften, Diss. Köln 2016

Kreutz, Peter, Das Objekt und seine Zuordnung, Baden-Baden 2017

Kriesel, David, A Brief Introduction to Neural Networks, Eigenpublikation Bonn 2007

Lämmel, Uwe/Cleve, Jürgen, Künstliche Intelligenz, 4. Aufl. München 2012

Lange, Hermann/Schiemann, Gottfried, Handbuch des Schuldrechts: Schadenersatz, 3. Aufl. Tübingen 2003

Langenscheidt Großwörterbuch Englisch, Berlin 2004

Larenz, Karl, Lehrbuch des Schuldrechts, Bd. 1 (Allgemeiner Teil), 14. Aufl. München 1987 (zitiert: Larenz, Lehrbuch des Schuldrechts I)

Larenz, Karl/Canaris, Claus-Wilhelm, Lehrbuch des Schuldrechts, Bd. II/2 (Besonderer Teil), 13. Aufl. München 1994 (zitiert: Larenz/Canaris, Lehrbuch des Schuldrechts $\mathrm{II} / 2)$

Larenz, Karl/Canaris, Claus-Wilhelm, Methodenlehre der Rechtswissenschaft, 3. Aufl. Berlin 1995 (zitiert: Larenz/Canaris, Methodenlehre)

Lauber-Rönsberg, Anne, Autonome »Schöpfung « - Urheberschaft und Schutzfähigkeit, GRUR 2019, 244-253

Laufs, Adolf, Der aktuelle Streit um das alte Problem der Willensfreiheit - Eine kritische Bestandsaufnahme aus juristischer Sicht, MedR 2011, 1-7

Lederer, Thomas L., Patentierung im Bereich Künstlicher Intelligenz, GRUR-Prax 2019, 152-154 
Leenen, Detlef, Ist das so richtig? - Typen von Defiziten der Zivilrechtsdogmatik, JuS 2008, 577-583

Lehmann, Matthias, Der Begriff der Rechtsfähigkeit, AcP 207 (2007), 225-255

Liu, Run-Qing/Lee, Young-Chan/Mu, Hong-Lei, Customer Classification and Market Basket Analysis Using K-Means Clustering and Association Rules: Evidence from Distribution Big Data of Korean Retailing Company, Knowledge Management Research 19/4 (2018), 59-76

Loenhoff, Jens, Implizites Wissen - Epistemologische und handlungstheoretische Perspektiven, Weilerswist 2012

Loh, Janina, Verantwortung und Roboterethik - ein kleiner Überblick - Teil 1, InTeR 2017, 220-226

Lohn, Jason D./Hornby, Gregory S./Linden, Derek S., Human-Competitive Evolved Antennas, AI EDAM 22 (2008), 235-247

Lowe, Ryan/Pow, Nissan/Serban, Iulian/Pineau, Joelle, The Ubuntu Dialogue Corpus: A Large Dataset for Research in Unstructured Multi-Turn Dialogue Systems, in: Proceedings of the 16th Annual Meeting of the Special Interest Group on Discourse and Dialogue (SIGDAL), 2015, S. 285-294 [arXiv:1506.08909] (zitiert: Lowe/Pow/ Serban/Pineau, in: SIGDAL)

Lüdemann, Volker, Connected Cars - Das vernetzte Auto nimmt Fahrt auf, der Datenschutz bleibt zurück, ZD 2015, 247-254

Luo, Jia/Lan, Edward, Fuzzy Logic Controllers for Aircraft Flight Control, in: Li, Hua Harry/Gupta, Madan M., Fuzzy Logic and Intelligent Systems, Norwell/Dordrecht 1995, S. 85-124 (zitiert: Luo/Lan, in: Li/Gupta)

Manber, Udi, Introduction to Algorithms - A Creative Approach, Reading (MA) 1989

Marly, Jochen, Praxishandbuch Softwarerecht, 7. Aufl. München 2018

Mayinger, Samantha Maria, Die künstliche Person - Untersuchung rechtlicher Veränderungen durch die Installation von Softwareagenten im Rahmen von Industrie 4.0, unter besonderer Berücksichtigung des Datenschutzrechts, Frankfurt am Main 2017

McCulloch, Warren S./Pitts, Walter, A Logical Calculus of the Ideas Immanent in Nervous Activity, Bulletin of the Mathematical Biophysics 5 (1943), 115-133

Medicus, Dieter, Probleme der Wissenszurechnung, in: Möglichkeiten der Wissenszurechnung - Karlsruher Forum 1994 (VersR Sonderheft), S. 4-16 (zitiert: Medicus, in: Möglichkeiten der Wissenszurechnung)

Medicus, Dieter/Lorenz, Stephan, Schuldrecht I - Allgemeiner Teil, 21. Aufl. München 2015

Medicus, Dieter/Petersen, Jens, Allgemeiner Teil des BGB, 11. Aufl. Heidelberg 2016

Mehrings, Josef, Vertragsabschluß im Internet - Eine neue Herausforderung für das »alte« BGB, MMR 1998, 30-33

Ménière, Yann/Piklajamaa, Heli, Künstliche Intelligenz in der Praxis des EPA, GRUR 2019, 332-336

Mittelstraß, Jürgen, Enzyklopädie Philosophie und Wissenschaftstheorie, Bd. 2 (C-F) 2. Aufl. Stuttgart 2005; Bd. 3 (G-Inn) 2. Aufl. Stuttgart 2008 (zitiert: Mittelstraß/ Bearbeiter: Beitrag) 
Montavon, Grégoire/Samek, Wojciech/Müller, Klaus-Robert, Methods for interpreting and unterstanding deep neural networks, Digital Signal Procession 73 (2018), 1-15

Moore, Edward F., The shortest path through a maze, in: Bell Telephone System. Technical publications, Bd. 3523, New York 1959, S. 1-8

Motwani, Rajeev, Randomized Algorithms, ACM Computer Surverys 8 (1996), 33-37

Moussa, Awaalom Daniel, Das Dogma vom formgerechten Zugang, Tübingen 2016

Müller-Hengstenberg, Claus D./Kirn, Stefan, Intelligente (Software-)Agenten: Eine neue Herausforderung unseres Rechtssystems - Rechtliche Konsequenzen der »Verselbstständigung « technischer Systeme, MMR 2014, 307-313

Münchener Kommentar zum Bürgerlichen Gesetzbuch, Bd. 1 (§§ 1-240, AllgPersönlR, ProstG, AGG), 8. Aufl. München 2018; Bd. 2 (§§ 241-310), 8. Aufl. München 2019; Bd. 3 ( $\S ~ 311-432)$, 8. Aufl. München 2019; Bd. 6 (§§ 705-853, PartGG, ProdHaftG), 7. Aufl. München 2017 (zitiert: MünchKommBGB/Bearbeiter)

Musielak, Hans-Joachim, Kausalität und Schadenszurechnung im Zivilrecht, JA 2013, 241-248

Musielak, Hans-Joachim, Zum Verhältnis von Wille und Erklärung. Eine Auseinandersetzung mit dem Tatbestand der Willenserklärung, AcP 211 (2011), 769-802

Nägerl, Joel/Neuburger, Benedikt/Steinbach, Frank, Künstliche Intelligenz: Paradigmenwechsel im Patentrecht, GRUR 2019, 336-341

Neumann, John von, First draft of a Report on the EDVAC, IEEE Annals of the History of Computing 15 (1993), Issue 4, 27-75 (Nachdruck)

Neuner, Jörg, Allgemeiner Teil des Bürgerlichen Rechts, 12. Aufl. München 2020

Neuner, Jörg, Was ist eine Willenserklärung?, JuS 2007, 881-888

Nguyen, Anh/Yosinski, Jason/Clune, Jeff, Deep Neural Networks are Easily Fooled: High Confidence Predictions for Unrecognizable Images, Paper 2015 [arXiv: 1412.1897]

Nha, Vi Tran Ngoc/Djahel, Soufiene/Murphy, John, A comparative study of vehicles' routing algorithms for route planning in smart cities, in: First International Workshop on Vehicular Traffic Management for Smart Cities (Proceedings), Dublin 2012, S. $1-6$

Nilsson, Nils J., Die Suche nach Künstliche Intelligenz: Eine Geschichte von Ideen und Erfolgen, Berlin 2014

Oechsler, Jürgen, Haftung beim Missbrauch eines eBay-Mitgliedskontos - Verantwortung für die freiwillige Überlassung und das Ausspähen von Verbindungsdaten, MMR 2011, 631-633

Omran, Mahamed G. H./Engelbrecht, Andries P./Salman, Ayed, An Overview of Clustering Methods, Intelligent Data Analysis 11 (2007), 583-605

Palandt, Otto, Bürgerliches Gesetzbuch, 79. Aufl. München 2020 (zitiert: Palandt) Bearbeiter)

Pasluosta, Cristian F./Chui, Alan W. L., Modulation of Grasping Force in Prosthetic Hands Using Neural Network-Based Predictive Control, in: Cartwright, Hugh, Artificial Neural Networks, New York 2015, S. 179-194 (zitiert: Pasluosta/Chiu, in: Cartwright) 
Patterson, David A./Hennessy, John L., Rechnerorganisation und Rechnerentwurf - Die Hardware/Software-Schnittstelle, 4. Aufl. München 2011

Paulheim, Heiko, Knowledge Graph Refinement: A Survey of Approaches and Evaluation Methods, The Semantic Web 2017, 489-508

Paulus, Christoph G./Zenker, Wolfgang, Grenzen der Privatautonomie, JuS 2001, 1-8

Paulus, David, Die automatisierte Willenserklärung, JuS 2019, 960-965

Pellissier-Tanon, Thomas/Weikum, Gerhard/Suchanek, Fabian, YAGO 4: A Reasonable Knowledge Base, The Semantic Web 2020, 583-596

Pérez Castaño, Arnaldo, Practial Artificial Intelligence, Havana 2018

Peters, Oliver/Runggaldier, Klaus, Algorithmen im Rettungsdienst, 4. Aufl. München 2014

Petersen, Jens, Allgemeiner Teil des BGB und Internet, JURA 2002, 387-390

Pieper, Fritz-Ulli, Künstliche Intelligenz: Im Spannungsfeld von Recht und Technik, InTeR 2018, 9-15

Pieper, Fritz-Ulli, Wenn Maschinen Verträge schließen: Willenserklärungen beim Einsatz von Künstlicher Intelligenz, GRUR-Prax 2019, 298-300

Priese, Lutz/Erk, Katrin, Theoretische Informatik: Eine umfassende Einführung, 4. Aufl. Berlin 2018

Rajkumar, Ragunathan/Lee, Insup/Sha, Lui/Stankovic, John, Cyber-Physical Systems: The Next Computing Revolution, in: Design Automation Conference, Proceeding Anaheim 2010, S. 731-736 (zitiert: Rajkumar/Lee/Sha/Stankovic, in: Design Automation Conference)

Reichwald, Julian/Pfisterer, Dennis, Autonomie und Intelligenz im Internet der Dinge, CR 2016, 208-212

Reusch, Philipp/Weidner, Niklas M., Future Law - Blockchain, Industrie 4.0, Internet of Things, Robotik, Frankfurt am Main 2018

Reuter, Dieter, Rechtsfähigkeit und Rechtspersönlichkeit - Rechtstheoretische und rechtspraktische Anmerkungen zu einem großen Thema, AcP 207 (2007), 673-717

Ribeiro, Marco Tulio/Singh, Sameer/Guestrin, Carlos, »Why Should I Trust You? «Explaining the Predictions of Any Classifier, Paper 2016 [arXiv:1602.04938]

Rich, Elaine, KI-Einführung und Anwendung, Hamburg 1988

Rich, Elaine/Knight, Kevin, Artificial Intelligence, 2nd Edition New York 1991

Riehm, Thomas, Von Drohnen, Google-Cars und Software-Agenten, ITRB 2014, 113 115

Riesenhuber, Karl, Privatautonomie - Rechtsprinzip oder »mystifizierendes Leuchtfeuer«, ZfPW 2018, 352-368

Roßnagel, Alexander, Aufgabe des Freiheitsschutzes in Rechtssetzung und -anwendung im Technikrecht, MMR 2020, 222-228

Rotsch, Thomas, Criminal Compliance, Baden-Baden 2015 (zitiert: Rotsch/Bearbeiter)

Russell, Stuart/Norvig, Peter, Artificial Intelligence - A Modern Approach, 2nd Edition Upper Saddle River 2003 (zitiert: Russell/Norvig ${ }^{2}$ ) 
Russell, Stuart/Norvig, Peter, Künstliche Intelligenz - Ein moderner Ansatz, 3. Aufl. Hallbergmoos 2012

Rüthers, Bernd/Fischer, Christian/Birk, Axel, Rechtstheorie, 10. Aufl. München 2018

Sahoo, Doyen/Pham, Quang/Lu, Jing/Hoi, Steven C. H., Online Deep Learning: Learning Deep Neural Networks on the Fly, Paper 2017 [arXiv:1711.03705]

Sandkühler, Hans Jörg, Enzyklopädie Philosophie, Bd. 1 (A-H) Hamburg 2010; Bd. 3 (Q-Z) Hamburg 2010 (zitiert: Sandkühler/Bearbeiter: Beitrag)

Savigny, Friedrich Carl von, System des heutigen römischen Rechts, Bd. 3, Berlin 1840

Schaaf, Nina/Huber, Marco F./Maucher, Johannes, Enhancing Decision Tree based Interpretation of Deep Neural Networks through L1-Orthogonal Regularization, Paper 2019 [arXiv: 1904.05394]

Schaub, Renate, Interaktion von Mensch und Maschine - Haftungs- und immaterialgüterrechtliche Fragen bei eigenständigen Weiterentwicklungen autonomer Systeme, JZ 2017, 342-349

Schirmer, Jan-Erik, Rechtsfähige Roboter?, JZ 2016, 660-666

Schirmer, Jan-Erik, Von Mäusen und Maschinen - Autonome Systeme in der Architektur der Rechtsfähigkeit, JZ 2019, 711-718

Schnapp, Friedrich E., Logik für Juristen, 7. Aufl. München 2016

Schönberger, Daniel, Deep Copyright: Up- and Downstream Questions Related to Artificial Intelligence (AI) and Machine Learning (ML), ZGE/IPJ 10 (2018), 35-58

Schricker, Gerhard/Loewenheim, Ulrich, Urheberrecht, 6. Aufl. München 2020 (zitiert: Schricker/Loewenheim/Bearbeiter)

Searle, John R., Minds, brains, and programs, The Behavioral and Brian Science 3 (1980), 417-424

Sester, Peter/Nitschke, Tanja, Software-Agenten mit Lizenz zum...? - Vertragsschluss und Verbraucherschutz beim Einsatz von Software-Agenten, CR 2004, 548-554

Shannon, Claude E., Programming a computer for playing chess, Philosophical Magazine, Ser. 7, Vol. 41 (1950), 256-275

Shu, Hai/Zhu, Hongtu, Sensitivity Analysis of Deep Neural Networks, Paper 2019 [arXiv: 1901.07152]

Siddhant, Aditya/Goyal, Anuj Kumar/Metallinou, Angeliki, Unsupervised Transfer Learning for Spoken Language Understanding in Intelligent Agents, Paper 2018, [arXiv:1811.05370]

Silver, David/Hubert, Thomas/Schrittwieser, Julian/Antonoglou, Ioannis/Lai, Matthew/ Guez, Arthur/Lanctot, Marc/Sifre, Laurent/Kumaran, Dharshan/Graepel, Thore/ Lillicrap, Timothy/Simonyan, Karen/Hassabi, Demis, Mastering Chess and Shogi by Self-Play with General Reinforcement Learning Algorithm, Paper 2017 [arXiv: 1712.01815] (zitiert: Silver/Hubert/Schrittwieser et al.)

Silver, David/Schrittwieser, Julian/Simonyan, Karen/Antonoglou, Ioannis/Huang, Aja/ Guez, Arthur/Hubert, Thomas/Baker, Lucas/Lai, Matthew/Bolton, Adrian/Chen, Yutian/Lillicrap, Timothy/Hui, Fan/Sifre, Laurent/Driesche, George van den/Graepel, Thore/Hassabis, Demis, Mastering the game of Go without human knowlede, Nature, Vol. 550 (2017), 354-359 (zitiert: Silver/Schrittwieser/Simonyan et al.) 
Söbbing, Thomas, Algorithmen und urheberrechtlicher Schutz, CR 2020, 223-228

Söbbing, Thomas, Fundamentale Rechtsfragen der künstlichen Intelligenz (AI Law), Frankfurt am Main 2019 (zitiert: Söbbing/Bearbeiter)

Söbbing, Thomas, Künstliche Intelligenz im HR-Recruiting-Prozess: Rechtliche Rahmenbedingungen und Möglichkeiten, InTeR 2018, 64-67

Sommerville, Ian, Software Engineering, 8. Aufl. München 2007

Sonnentag, Michael, Vertragliche Haftung bei Handeln unter fremdem Namen im Internet, WM 2012, 1614-1620

Sosnitza, Olaf, Das Internet der Dinge - Herausforderungen oder gewohntes Terrain für das Zivilrecht?, CR 2016, 764-772

Specht, Louisa, Zum Verhältnis von (Urheber-)Recht und Technik, GRUR 2019, 253259

Specht, Louisa/Herold, Sophie, Roboter als Vertragspartner? - Gedanken zu Vertragsabschlüssen unter Einbeziehung automatisiert und autonom agierender Systeme, MMR 2018, 40-44

Spindler, Gerald, Kausalität im Zivil- und Wirtschaftsrecht, AcP 208 (2008), 283-344

Spindler, Gerald, Roboter, Automaten, künstliche Intelligenz, selbst-steuernde Kfz Braucht das Recht neue Haftungskategorien?, CR 2015, 766-776

Spindler, Gerald/Schuster, Fabian, Recht der elektronischen Medien, 4. Aufl. München 2019 (zitiert: Spindler/Schuster/Bearbeiter)

Stadler, Astrid, Allgemeiner Teil des BGB, 19. Aufl. München 2017

Stathopoulos, Michael, Zur Methode der Auslegung der Willenserklärung, in: Festschrift für Larenz zum 70. Geburtstag, S. 357-372 (zitiert: Stathopoulos, in: FS Larenz)

Staudinger, Julius von, Kommentar zum Bürgerlichen Gesetzbuch, Buch 1: Allgemeiner Teil: Einl. BGB, §§ 1-14 BGB; VerschG, Berlin 2018; §§ 90-124, 130-133 BGB, Berlin 2016; §§ 139-163 BGB, Berlin 2015; §§ 164-240 BGB, Berlin 2019; Buch 2: Schuldrecht: $\S \S 249-254$ BGB, Berlin 2016; §§ 255-304 BGB, Berlin 2019; § 823 AD BGB, Berlin 2016; §§ 823 E-I, 824, 825 BGB, Berlin 2009; §§ 826-829 BGB, ProdHaftG, Berlin 2018 (zitiert: Staudinger/Bearbeiter)

Steiniger, Andreas, Die Jurisprudenz auf Erkenntnissuche? - Ein Plädoyer für eine Neuorientierung der Rechtswissenschaft, NJW 2015, 1072-1077

Stiemerling, Oliver, »Künstliche Intelligenz« - Automatisierung geistiger Arbeit, Big Data und das Internet der Dinge, CR 2015, 762-765

Stockmar, Kendra/Wittwer, Alexander, Die Pflicht zur Empfangsbestätigung von elektronischen Bestellungen im Spiegel der Rechtsprechung, CR 2005, 118-126

Stoklas, Jonathan, Bessere Grenzkontrollen durch Künstliche Intelligenz, ZD-Aktuell 2018, 06363

Stübinger, Stephan, Das »idealisierte« Strafrecht, Frankfurt a.M. 2008

Sun, Youcheng/Huang, Xiaowei/Kroening, Daniel/Sharp, James/Hill, Matthew/Ashmore, Rob, Testing Deep Neural Networks, Paper 2019 [arXiv: 1803.04792] (zitiert: Sun/Huang/Kroening et al.) 
Sutschet, Holger, Anforderungen an die Rechtsgeschäftslehre im Internet - Bid Shielding, Shill Bidding und Mr. Noch Unbekannt, NJW 2014, 1041-1046

Tamm, Marina/Tonner, Klaus/Brönneke, Tobias, Verbraucherrecht, 3. Aufl. BadenBaden 2020 (zitiert: Tamm/Tonner/Brönneke/Bearbeiter)

Tanenbaum, Andrew S./Wetherall, David J., Computernetzwerke, 5. Aufl. Hallbergmoos 2012

Taupitz, Jochen, Wissenszurechnung nach englischem und deutschem Recht, in: Möglichkeiten der Wissenszurechnung - Karlsruher Forum 1994 (VersR Sonderheft), S. 16-30 (zitiert: Taupitz, in: Möglichkeiten der Wissenszurechnung)

Taupitz, Jochen/Kritter, Thomas, Electronic Commerce - Probleme bei Rechtsgeschäften im Internet, JuS 1999, 839-846

Tegmark, Max, Leben 3.0: Menschen sein im Zeitalter Künstlicher Intelligenz, 3. Aufl. Berlin 2019

Tesauro, Gerald, Programming backgammon using self-teaching neural nets, Artificial Intelligence 134 (2002), 181-199

Teubner, Gunther, Digitale Rechtssubjekte? AcP 218 (2018), 155-205

The Oxford English Dictionary, Vol. I (A-Bazouki) 2nd Edition Oxford 1989; Vol. VII (Hat-Intervacuum) 2nd Edition Oxford 1989

Thomas, Oliver, Fuzzy Process Engineering, Wiesbaden 2009

Timm, Wolfram, Die Rechtsfähigkeit der Gesellschaft bürgerlichen Rechts und ihre Herausforderungen, NJW 1995, 3209-3218

Trobec, Roman/Slivnik, Boštjan/Bulić, Patricio/Robič, Borut, Introduction to Parallel Computing: From Algorithms to Programming on State-of-the-Art Platforms, Cham 2018

Unlusoy, Y. Samim /Yazicioglu, Yigit, A fuzzy logic controlled Anti-lock Braking System (ABS) for improved braking performance and directional stability, Int. J. Vehicle Design, Vol 48(3) (2008), 1-18

Wagner, Gerhard, Produkthaftung für autonome Systeme, AcP 217 (2017), 707-765

Wagner, Gerhard, Roboter als Haftungssubjekte? Konturen eines Haftungsrechts für autonome Systeme, in: Faust, Florian/Schäfer, Hans-Bernd, Zivilrechtliche und rechtsökonomische Probleme des Internet und der künstlichen Intelligenz, Tübingen 2019, S. 1-39 (zitiert: Wagner, in: Faust/Schäfer)

Wagner, Gerhard, Robot, Inc.: Personhood for Autonomous Systems?, 88 Fordham L.Rev. 591-612 (2019)

Wagner, Gerhard, Wissenszurechnung: Rechtsvergleichende und rechtsökonomische Grundlagen, ZHR 181 (2017), 203-272

Wahlster, Wolfgang, Künstliche Intelligenz als Grundlage autonomer Systeme, Informatik-Spektrum 40 (2017), 409-418

Waldstein, Wolfgang, Willensfreiheit und rechtliche Ordnung, in: Festschrift Fritz Schwind zum 65. Geburtstag, Wien 1978, S. 329-353 (zitiert: Waldstein, in: FS Schwind)

Wallace, Danny P., Knowledge Management: Historical and Cross-Disciplinary Themes, Westport 2007 
Wandt, Manfred, Gesetzliche Schuldverhältnisse, 9. Aufl. München 2019

Weber, Robert/Kiefner, Alexander/Jobst, Stefan, Künstliche Intelligenz und Unternehmensführung, NZG 2018, 1131-1136

Wiater, Werner, Wissensmanagement - Eine Einführung für Pädagogen, Wiesbaden 2007

Wiebe, Andreas, Die elektronische Willenserklärung, Tübingen 2002

Wiendahl, Hans-Hermann, Auftragsmanagement der industriellen Produktion, Berlin 2011

Wilkesmann, Uwe/Rascher, Ingolf, Wissensmanagement - Analyse und Handlungsempfehlungen, Düsseldorf 2003

Wirth, Niklaus, Algorithmen und Datenstrukturen, 3. Aufl. Stuttgart 1983

Wolf, Manfred, Schuldnerhaftung bei Automatenversagen, JuS 1989, 899-902

Wolf, Manfred/Neuner, Jörg, Allgemeiner Teil des Bürgerlichens Rechts, 11. Aufl. München 2016 (zitiert: Wolf/Neuner ${ }^{11}$ )

Wolf, Marty J./Miller, Keith W./Grodzinsky, Frances S., Why we should have seen that coming: comments on Microsoft's tay »experiment«, and wider implications, ACM SIGCAS Computers \& Society, Volume 47 No. 3 (2017), 47-64

Wolff, Hans J., Organschaft und juristische Personen, Bd. 1 (Juristische Person und Staatsperson), Berlin/Aalen 1933/1968

Wong, Ka-Chun, Evolutionary Algorithms: Concepts, Designs, and Applications in Bioinformatics, Paper 2015 [arXiv:1508.00468]

Wulf, Hans Markus/Burgenmeister, Clemens, Industrie 4.0 in der Logik - Rechtliche Hürden beim Einsatz neuer Vernetzungs-Technologien, CR 2015, 404-412

Yosinski, Jason/Clune, Jeff/Nguyen, Anh/Fuchs, Thomas/Lipson, Hod, Understanding Neural Networks Through Deep Visualization, Paper 2015 [arXiv: 1506.06579] (zitiert: Yosinski/Clune/Nguyen et al.)

Zech, Herbert, Information als Schutzgegenstand, Tübingen 2012

Zech, Herbert, Künstliche Intelligenz und Haftungsfragen, ZfPW 2019, 198-219

Zech, Herbert, Zivilrechtliche Haftung für den Einsatz von Robotern - Zuweisung von Automatisierungs- und Autonomierisiken, in: Gless, Sabine/Seelmann, Kurt, Intelligente Agenten und das Recht, Baden-Baden 2016, S. 163-204 (zitiert: Zech, in: Gless/Seelmann)

Zhou, Bolei/Kosla, Aditya/Lapedriza, Agata/Oliva, Aude/Torralba, Antonio, Learning Deep Features for Discriminative Localization, Paper 2015 [arXiv: 1512.04150] (zitiert: Zhou/Khosla/Lapedriza et al.)

Zimmermann, Reinhard, »Heard melodies are sweet, but those unheard are seweeter..." - Condictio tacita, implied condition und die Fortbildung des europäischen Vertragsrechts, AcP 193 (1993), 121-173

Zoph, Berret/Vasudeven, Vijay/Shlens, Jonathon/Le, Quoc V., Learning Transferable Architectures for Scalable Image Recognition, Paper 2018 [arXiv: 1707.07012] 


\section{Stichwortverzeichnis}

Agent 30

Algorithmus

- Definition 53

- randomisierter 54

- Weg zum Programm 55

Begriff 21, 30

Bereicherungsrecht 112

Big Data 44

Blanketterklärung 111

Clustering-Algorithmen 38

Computer 26

Computersoftware 30, 52, 73

Computersystem 73

Cyber-Physisches-System 73

Deliktsrecht 117, 119, 127

Denken 26

Determiniertheit 59

Determinismus

- informatischer 59

- konvergierender 60

- philosophischer 58

- rechtlicher 58

- stochastischer 60

Entscheidungsbaum 35

ePerson 77

Erfüllungsgehilfe 126

Erklärbarkeit 62, 120

Evolutionärer Algorithmus 42

Expertensystem 40

Explainable AI 65, 120
Fehler 62, 115, 117, 120

Fuzzylogik 39

Handlung

- Definition 26

- menschliche 26

- unerlaubte 117, 119, 127

- von Computern 26, 64

Haftung

- außervertragliche 115, 120

- Fahrlässigkeit 117, 120

- Kausalität 128

- Rechtsschein 94, 100

- vertragliche 115

- Vorsatz 117, 119

Künstliche Intelligenz

- Arten und Methoden 34, 45

- Begriff 21, 30

- Definition 30

Künstliches neuronales Netz

- Anwendungsphase 49

- Black-Box 51

- Definition 45

- Online-Learning 49

- Trainingsphase 47

Lernen

- Machine Learning 34, 35, 38, 60

- Online-Learning 49

- Trainingsphase 47

- Weiterlernen 49 
Methoden 34

Modell 52

Multi-Agenten-System 52

Produkthaftung

- deliktische 120

- verschuldensunabhängige 125

Rechtsfähigkeit

- Begriff 75

- Folgen 112, 132

- teilweise 77

- vollständige 77

Rechtsobjekt 73

Rechtssubjekt 75

Roboter 73

Semantic Web System 40

Software 30, 52, 73

Stellvertretung 81, 94, 99, 112

Suchstrategie 34

Terminierung 59

Verhalten

- Definition 26

- einer KI 62

- unvorhersehbares 64

- Zurechnung 71

Verrichtungsgehilfe 127

Verschulden 117, 119

Vertrag 81, 89, 97, 100, 104

Vorhersehbarkeit 62, 120, 130

Wille 26, 60, 83

Willenserklärung

- Abgabe 90, 100, 105, 111

- Anfechtung 83, 88, 112

- Angebot, autonomes 105
- Annahme, automatische 97

- Annahme, autonome 108

- Annahmefrist 97, 108

- automatische 96

- autonome 104

- Komplex 96

- elektronische 81, 90, 100

- regelbasierte 99

- Zugang 93, 108

Willensfreiheit 58

Wissen

- Definitionen 66

- Repräsentation 66

- Zurechnung 133

Zurechnung

- Grundlagen 72

- Subjekte 72

\section{2}

